Universidade DE SÃo PAUlo

Instituto de Física de SÃo Carlos

Departamento de Física e Informática

\title{
Transporte Quântico em Spintrônica: corrente e shot noise via funções de Green de não-equilíbrio
}

Fabrício Macedo de Souza

Tese apresentada ao Instituto de Física de São Carlos, Universidade de São Paulo, para obtenção do Título de Doutor em Ciências: Física Básica

Orientador: Prof. Dr. José Carlos Egues

SÃo CARLOS

2004 


\begin{abstract}
Souza, Fabrício Macedo
Transporte Quântico em Spintrônica: corrente e shot noise via funções de Green de não-equilíbrio / Fabrício Macedo de Souza. - São Carlos, 2004.

p.166

Tese (Doutorado) - Universidade de São Paulo. Instituto de Física de São Carlos. Programa de Pós-graduação em Física.

Orientador: Prof. Dr. José Carlos Egues.

1. spintronica, 2. formalismo de Keldysh, 3. corrente e shot noise em pontos quânticos, 4. transporte polarizado.
\end{abstract}

Esta tese foi preparada em ${ }^{A} \mathrm{~T}_{\mathrm{E}} \mathrm{X}$. 
Dedico esta tese à minha esposa Ana Júlia e à minha filha Isabelle. 


\section{Agradecimentos}

- Ao meu orientador, Prof. Dr. José Carlos Egues, por ter me guiado ao longo do meu doutorado, e contribuído para a minha formação científica.

- Ao Prof. Dr. Antti-Pekka Jauho pela co-orientação e por ter me acolhido calorosamente em seu grupo de pesquisa no Centro de Microeletrônica da Universidade Técnica da Dinamarca (DTU).

- Aos colegas Dr. Henrique J. P. Freire (USP), Dr. Vivaldo Campos (USP), Prof. Dr. Klaus W. Capelle (USP), Dr. Andrea Donarini (DTU), pelas frutíferas discussões e amizade.

- A nossa querida secretária do grupo de física teórica Maria Cristina V. L. Silva pelo apóio técnico/administrativo.

- A todo o pessoal, alunos, professores e funcionários, do IFSC-USP que contribuíram para o desenvolvimento deste trabalho.

Fica ainda registrada a minha gratidão a Deus, pela sabedoria para realizar esse trabalho. A minha querida esposa Ana Julia, por todo carinho, apoio e compreensão. A minha filha Isabelle, pela alegria do seu nascimento. A meus pais, José e Graça, que sempre estiveram presentes nos bastidores da minha vida acadêmica, e a minha irmã Alessandra, por todos os aconselhamentos ao longo desses anos.

Este trabalho foi financiado pelas agências FAPESP e CAPES. 


\section{Resumo}

Estudamos transporte quântico dependente de spin em sistemas de ponto e de poço quântico acoplados a contatos magnéticos. O primeiro passo do nosso estudo foi a dedução de fórmulas originais para a corrente e para o ruído em sistemas com tunelamento dependente de spin, através do formalismo de funções de Green de não-equilíbrio. As equações deduzidas reproduzem casos limites da literatura - em particular as formulas de Landauer-Büttiker. Posteriormente aplicamos essas fórmulas para estudar três sistemas distintos: (1) ponto quântico acoplado a contatos ferromagnéticos, (2) um ponto quântico acoplado a multiplos terminais ferromagnéticos, e (3) um poço quântico acoplado a terminais de semicondutor magnético diluído (DMS). No sistema (1) consideramos os alinhamentos paralelo (P) e anti-paralelo (AP) entre as magnetizações dos terminais. Nesse sistema levamos em conta interação de Coulomb e espalhamento de spin no ponto quântico. Com as fórmulas para corrente e ruído deduzidas aqui, encontramos, por exemplo, que a interação de Coulomb, combinada com o magnetismo dos eletrodos, leva a um bloqueio de Coulomb dependente de spin. Esse efeito por sua vez leva a uma polarização da corrente que pode ser modulada (intensidade e sinal) através de uma tensão externa. Também encontramos que o espalhamento de spin leva a comportamentos contrastantes entre corrente e ruído. Enquanto a corrente na configuração AP aumenta com a taxa de espalhamento de spin $R$, o ruído nessa mesma configuração é suprimido para uma certa faixa de valores de $R$. Um outro efeito interessante que observamos foi a possibilidade de se suprimir o ruído térmico através de uma tensão de porta. Para o sistema (2) mostramos que é possível injetar corrente $\uparrow$-polarizada no ponto quântico e coletar simultaneamente correntes $\uparrow$ e $\downarrow$ polarizadas em terminais diferentes. Além disso, a corrente ao passar do reservatório emissor para um dos reservatórios coletores tem a sua polarização intensificada. Portanto esse sistema pode operar como inversor e amplificador de polarização de corrente. Por último, analisamos os efeitos de terminais DMS e quantização de Landau (na presença de um campo magnético externo) sobre a corrente e o ruído no sistema (3). Encontramos que o efeito Zeeman gigante nos terminais DMS, gerado pela interação de troca $s$ - $d$, leva a uma polarização da corrente. Em particular, para uma certa faixa de tensão o efeito Zeeman gigante resulta na completa supressão de uma dada componente de spin no transporte. Com isso é possível controlar a polarização da corrente através de uma tensão externa. Também observamos oscilações na corrente, no ruído e no fator de Fano como função do campo magnético. 


\section{Abstract}

We study spin dependent quantum transport in quantum dots and quantum well devices attached to magnetic leads. We first derive general formulas, including electron-electron interaction and spin flip, for both current and noise, using the nonequilibrium Green function technique (Keldysh). From our equations we regain limiting cases in the literature - in particular the Landauer-Büttiker formula when we neglect electron-electron interaction. We apply these formulas to study three distinct systems: (1) a quantum dot attached to two ferromagnetic leads, (2) a quantum dot linked to many ferromagnetic leads, and (3) a quantum well coupled to dilute magnetic semiconductor (DMS) terminals. In the first system we consider both parallel (P) and antiparallel (AP) ferromagnetic alignments of the leads. Coulomb interaction and spin flip scattering are also taken into account. With the formulas for the current and the noise derived here, we find, for instance, that the Coulomb interaction, combined with the magnetism of the electrodes, gives rise to a spin-dependent Coulomb blockade. This effect allows the control (intensity and sign) of the current polarization via the bias voltage. We also observe that spin flip scattering yields contrasting behavior between current and shot noise. While the current in the AP configuration increases with the spin flip, the shot noise becomes suppressed for a range of spin flip rates. Another interesting finding is the possibility to suppress the thermal noise via a gate voltage. For the dot coupled to three magnetic leads, we show that it is possible to inject current $\uparrow$-polarized into the dot from the FM emitter, detect simultaneously $\uparrow$ - and $\downarrow$-polarized currents at distinct collectors. In addition, we find that the current has its polarization amplified when going from the emitter to one of the collectors. Therefore we have a device that operates as both as current polarization inverter and amplifier. Finally, we analyze the effects of DMS leads and Landau quantization on the current and noise of system (3). We find that the giant Zeeman effect in the DMS leads, due to the it s-d exchange interaction, gives rise to a spin polarized current, and for a particular bias voltage range, full suppression of one spin component. This gives rise to the possibility of tuning the current polarization via the bias voltage. We also observe oscillations in the current, the noise and the Fano factor as a function of the magnetic field. 


\section{Conteúdo}

Resumo iv

Abstract $\quad$ V

Lista de Figuras viii

1 Introdução 1

1.1 Pontos quânticos . . . . . . . . . . . . . . . . . . . 1

1.2 Transporte quântico . . . . . . . . . . . . . . . . . 2

1.3 Eletrônica de spin . . . . . . . . . . . . . . . . 5

1.4 Ruído . . . . . . . . . . . . . . . . . . . . 6 6

1.5 Nossos resultados . . . . . . . . . . . . . . . . . . . 8

2 Introdução às Funções de Green de Não-Equilíbrio 11

2.1 Formulação do problema . . . . . . . . . . . . . . . . . . . . . . . . 11

2.2 A função de Green ordenada no contorno . . . . . . . . . . . . . . . 13

2.3 A origem do contorno de integração . . . . . . . . . . . . . . . . . . 14

2.4 As funções de Green $G^{t}, G^{\tilde{t}}, G^{<}$e $G^{>} \ldots \ldots$. . . . . . . . . . . 15

2.5 Continuação Analítica . . . . . . . . . . . . . . . . . 16

2.6 Equação de Keldysh ...................... 19

3 Formulação do transporte para o sistema FM-PQ-FM 21

3.1 Introdução . . . . . . . . . . . . . . . . . . . . . . . . . . . 21

3.2 Modelo . . . . . . . . . . . . . . . . . . . . . 22

3.3 Corrente . . . . . . . . . . . . . . . . . . . . 23

3.4 Expressão para a corrente no caso $U=0$ e $R=0$. . . . . . . . . 26

3.5 Ruído . . . . . . . . . . . . . . . . . . . . 27

3.6 Expressão para o ruído no caso $U=0$ e $R=0$. . . . . . . . . . 29

3.7 Funções de Green do Ponto Quântico . . . . . . . . . . . . . . . . . 30

4 Resultados para o sistema FM-PQ-FM 35

4.1 Procedimento Numérico . . . . . . . . . . . . . . . . . . . . 36

4.2 Função Espectral . . . . . . . . . . . . . . . . . . . . . . . . . . 37 
4.2.1 Efeitos do ferromagnetismo dos eletrodos sobre a função espectral . . . . . . . . . . . . . . . . . . . 38

4.2.2 Efeitos do espalhamento de spin sobre $A(\epsilon)$. . . . . . . . 39

4.2.3 Efeitos da interação de Coulomb sobre a função espectral . . 41

4.3 Ocupação eletrônica no ponto quântico . . . . . . . . . . . . . . . 41

4.3.1 Ocupação eletrônica no caso simétrico $p_{L}=p_{R}$. . . . . . . 42

4.3.2 Ocupação eletrônica no caso assimétrico $p_{L} \neq p_{R} \ldots \ldots$. . . 47

4.3.3 Ocupações como função de $\mathrm{U}[\mathrm{eV}=4 \mathrm{meV}, R=0]$. . . . . 49

4.3.4 Efeitos do espalhamento de spin sobre $n_{\uparrow}$ e $n_{\downarrow} \ldots \ldots$. . . . 51

4.4 Corrente . . . . . . . . . . . . . . . . . . 51

4.4.1 Corrente e sua polarização no caso simétrico $\left[p_{L}=p_{R}\right]$. . . 52

4.4.2 Corrente e sua polarização no caso assimétrico $\left[p_{L} \neq p_{R}\right]$. . 54

4.5 Fator de supressão de corrente . . . . . . . . . . . . . . 56

4.6 Corrente e magnetoresistência de tunelamento . . . . . . . . . . . . 57

4.7 Efeitos de Interferência . . . . . . . . . . . . . . . . . . . . . . . 59

4.7.1 Dedução de uma expressão analítica para a corrente $(U=0) . \quad 59$

4.7 .2 Caso $U \neq 0$. . . . . . . . . . . . . . . . . . . . . . . 61

4.8 Modelando o espalhamento de spin através de uma associação de resistores . . . . . . . . . . . . . . . . . . . . 64

4.9 Ruído . . . . . . . . . . . . . . . . . . . . . 67

4.9.1 Efeitos do bloqueio de Coulomb sobre o ruído . . . . . . . . 67

4.9.2 Fator de supressão de ruído. . . . . . . . . . . . . . . . . . . 69

4.9.3 Ruído térmico . . . . . . . . . . . . . . . . . . . . 70

4.9.4 Shot noise na presença de espalhamento de spin . . . . . . 73

4.9.5 Fator de Fano . . . . . . . . . . . . . . . . . . . . . 77

4.9.6 Expressão analítica para o ruído . . . . . . . . . . . . . 77

5 Gerador simultâneo de correntes com polarizações $\uparrow$ e $\downarrow$

5.1 Modelo . . . . . . . . . . . . . . . . . . . . . . . 82

5.2 Corrente . . . . . . . . . . . . . . . . . . 83

5.3 Funções de Green do ponto quântico . . . . . . . . . . . . . . . . . . 84

5.3 .1 Auto-energias . . . . . . . . . . . . . . . 85

5.3.2 Função de Green retardada e equação de Keldysh ..... . 86

5.4 Forma explícita da corrente . . . . . . . . . . . . . 86

5.5 Resposta linear . . . . . . . . . . . . . . . . . . . . 88

5.6 Sistema envolvendo três terminais . . . . . . . . . . . . . . . 88

5.6.1 Corrente no sistema de três terminais . . . . . . . . . . . . . 91

5.7 Função Espectral . . . . . . . . . . . . . . . . . . . . . . . . 92

5.8 Parâmetros e metodologia de cálculo . . . . . . . . . . . . . . . 92

5.9 Resultados . . . . . . . . . . . . . . . . . . . 92

5.9.1 Corrente e polarização versus voltagem . . . . . . . . . . . . 92 
5.9.2 Dependência das correntes $I_{\uparrow}$ e $I_{\downarrow}$ como função da polarização dos eletrodos . . . . . . . . . . . . . . . . . . . . . 99

5.10 Associação de resistores . . . . . . . . . . . . . . . . . . . 105

6 Poço quântico acoplado a contatos semimagnéticos 107

6.1 Propriedades magnéticas e a interação de troca . . . . . . . . . . . 108

6.2 Modelo . . . . . . . . . . . . . . . . . . . . . . . . . . . 110

6.3 Corrente . . . . . . . . . . . . . . . . . . . 111

6.4 Shot noise . . . . . . . . . . . . . . . . . . . . . . . . 112

6.5 Funções de Green do poço quântico . . . . . . . . . . . . . . . . . . . 114

6.6 Conexão com Landauer-Büttiker . . . . . . . . . . . . . . . . . . . . 115

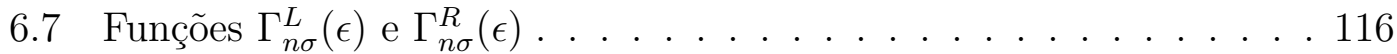

6.8 Parâmetros e Metodologia . . . . . . . . . . . . . . . . . 116

6.9 Resultados . . . . . . . . . . . . . . . . . . . . . 117

6.9.1 Densidade de estados . . . . . . . . . . . . . . . 117

6.9.2 Corrente e ruído como função de $V$. . . . . . . . . . . . . . 121

6.9.3 Corrente e ruído versus B . . . . . . . . . . . . . . . . . . 124

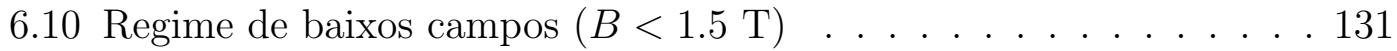

$\begin{array}{llr}7 & \text { Conclusão } & 137\end{array}$

$\begin{array}{lr}\text { Apêndices } & 140\end{array}$

$\begin{array}{ll}\text { A Cálculo da função de Green } G_{i \mathbf{k} \sigma \eta}^{<}(t, t) & 141\end{array}$

$\begin{array}{ll}\text { B Cálculo da expressão para o ruído } & 145\end{array}$ 


\section{Lista de Figuras}

1.1 Ilustração esquemática de um ponto quântico acoplado a eletrodos . 2

1.2 Perfil do potencial de um ponto quântico acoplado a terminais . . . 4

2.1 Contorno de tempo utilizado na definição da função de Green ordenada no contorno . . . . . . . . . . . . . . . . . . . 14

3.1 Ilustração de um sistema formado por um ponto quântico acoplado a dois terminais ferromagnéticos . . . . . . . . . . . . . . 21

4.1 Esquema do procedimento numérico utilizado no cálculo auto-consistente 37

4.2 Função espectral $A(\epsilon)$ nas configurações P e AP . . . . . . . . . . . 39

4.3 Função espectral resolvida em spin . . . . . . . . . . . . . . . . 40

4.4 Função espectral para diferentes valores do parâmetro de espalhamento de spin . . . . . . . . . . . . . . . . . . . . 4 40

4.5 Função espectral para diferentes valores de $U$. . . . . . . . . . . . 42

4.6 Ocupações $n_{\uparrow}$ e $n_{\downarrow}$ e magnetização contra tensão $e V$. . . . . . . . . . 44

4.7 Diagrama de níveis de energia para o sistema de dois terminais acoplados a um ponto quântico . . . . . . . . . . . . . . 46

4.8 Curvas análogas as da Fig.(4.6) exceto pelos valores de $p_{L}$ e de $p_{R}$ utilizados . . . . . . . . . . . . . . . . . . . 48

4.9 Ocupações $n_{\uparrow}$ e $n_{\downarrow}$ versus $U \ldots \ldots$. . . . . . . . . . . 50

4.10 Ocupações $n_{\uparrow}$ e $n_{\downarrow}$ para diferentes parâmetros de espalhamento de

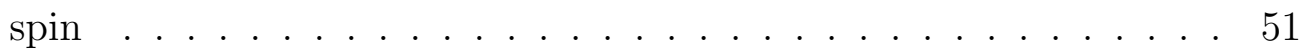

4.11 Ocupações $n_{\uparrow}$ e $n_{\downarrow}$, correntes $I_{\uparrow}$ e $I_{\downarrow}$, e polarização nas configurações $\mathrm{P}$ e AP como função da voltagem para $p_{L}=p_{R}=0.4 \ldots . . . \quad 53$

4.12 Ocupações $n_{\uparrow}$ e $n_{\downarrow}$, correntes $I_{\uparrow}$ e $I_{\downarrow}$, e polarização nas configurações $\mathrm{P}$ e AP como função da voltagem para $p_{L}=0.23$ e $p_{R}=0.35 \ldots . \quad 55$

4.13 Correntes na configuração paralela $I_{P}$, anti-paralela $I_{A P}$ e magnetoresistência de tunelamento . . . . . . . . . . . . . . . . . 58

4.14 Função espectral $A(\epsilon)$, corrente anti-paralela $I_{A P}$ e corrente no modelo de interferência . . . . . . . . . . . . . . . . . 63

4.15 Associação de resistores . . . . . . . . . . . . . . . . . 65

4.16 Correntes $I_{P}$ e $I_{A P}$ como função do parâmetro de espalhamento de

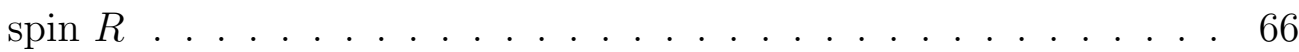


4.17 Corrente e ruído resolvidos em spin como função da tensão $V, U=0$ (curvas pretas), $U=1 \mathrm{meV}$ (curvas vermelhas), $p_{L}=p_{R}=0.4 \ldots 68$

4.18 Corrente e ruído resolvidos em spin como função da tensão $V, U=0$ (curvas pretas), $U=1 \mathrm{meV}$ (curvas vermelhas), $p_{L}=0.23$, e $p_{R}=0.3569$

4.19 Ruido térmico como função da posição do nível $\epsilon_{d}$. . . . . . . . . . 71

4.20 Função espectral (linhas vermelhas) para três valores de $\epsilon_{d}$. . . . . 72

4.21 Ruído térmico como função da posição do nível $\epsilon_{d}$ para diferentes valores do parâmetro de espalhamento de spin $R$. . . . . . . . . 73

4.22 Ruído versus tensão nos alinhamentos (a) paralelo e (b) antiparalelo 74

4.23 Corrente, ruído e fator de Fano como função de $R$. . . . . . . . . . 76

4.24 Sistema de três barreiras análogo ao sistema FM-PQ-FM no caso AP 100\% polarizado com espalhamento de spin . . . . . . . . . 79

4.25 Fator de Fano como função de $R$ para a configuração AP $100 \%$ polarizada . . . . . . . . . . . . . . . . . 81

5.1 Sistema composto de vários terminais ferromagnéticos acoplados a um ponto quântico . . . . . . . . . . . . . . . . . . . 83

5.2 Sistema constituído por três terminais ferromagnéticos acoplados a um ponto quântico . . . . . . . . . . . . . . . . . . . . . . 89

5.3 Correntes $I_{\uparrow}$ e $I_{\downarrow}$ como função da tensão externa, nos três terminais e nas três configurações magnéticas analisadas . . . . . . . . . . . 95

5.4 Polarização da corrente, $\wp=\left(I_{\uparrow}-I_{\downarrow}\right) /\left(I_{\uparrow}+I_{\downarrow}\right)$, nos vários terminais, como função da tensão externa aplicada . . . . . . . . . . . . . 96

5.5 Funções espectrais $A_{\uparrow}(\epsilon)$ (linhas pretas) e $A_{\downarrow}(\epsilon)$ (linhas vermelhas) nas três configurações magnéticas dos terminais . . . . . . . . . . 97

5.6 Magneto resistência de tunelamento no sistema de três terminais ferromagnéticos. . . . . . . . . . . . . . . . . . . . . . . . . 99

5.7 Correntes $I_{\uparrow}$ e $I_{\downarrow}$ como função de $p_{i}$-configuração $1 \ldots$. . . . . . . 102

5.8 Correntes $I_{\uparrow}$ e $I_{\downarrow}$ como função de $p_{i}$-configuração $2 \ldots$. . . . . . . 103

5.9 Correntes $I_{\uparrow}$ e $I_{\downarrow}$ como função de $p_{i}$-configuração 3 . . . . . . . . . . 104

5.10 Associação de resistores . . . . . . . . . . . . . . 106

6.1 Spin médio das impurezas de Mn na liga ternária de ZnMnSe como função do campo magnético . . . . . . . . . . . . . . . . . . . . . . 109

6.2 Densidade de estados dos terminais e do poço quântico. . . . . . . . 118

6.3 Densidade de estados para $n=0$. . . . . . . . . . . . . 120

6.4 Corrente, ruído e fator de Fano como função da voltagem . . . . . . 122

6.5 Níveis de Landau e potenciais químicos como função da voltagem . 123

6.6 Corrente, ruído e fator de Fano como função do campo magnético 128

6.7 Níveis de Landau como função do campo magnético . . . . . . . . . 129

6.8 Densidade de estados correspondente ao nível de Landau $n=0$. . . 130 
6.9 Funções $\Gamma\left(\epsilon_{z}\right)^{L}, \Gamma\left(\epsilon_{z}\right)^{R}, A_{\sigma}\left(\epsilon_{z}\right)$ e coeficiente de transmissão $T\left(\epsilon_{z}\right)$ como função da energia longitudinal $\epsilon_{z}$. . . . . . . . . . . . . . . 135

6.10 Figura semelhante a Fig. 6.9, exceto pela tensão externa adotada $(e V=60 \mathrm{meV}) \ldots \ldots \ldots \ldots \ldots$ 


\section{Capítulo 1}

\section{Introdução}

\subsection{Pontos quânticos}

Com o desenvolvimento das técnicas de miniaturização de dispositivos (e.g. litografia, MBE), uma variedade de novos sistemas vem surgindo, cujas dimensões giram em torno de dezenas a centenas de nanometros. Assim, sistemas quânticos que antes pareciam puramente acadêmicos, como os problemas de tunelamento através de uma ou duas barreiras [1], hoje em dia são produzidos rotineiramente nos laboratórios. Nessa escala, uma descrição quântica dos fenômenos físicos torna-se fundamental para compreender propriedades como o transporte elétrico.

Em particular, um sistema nanoscópico que vem recebendo crescente atenção é constituído de reservatórios de elétrons (terminais) acoplados, via barreiras de tunelamento, a um sistema de dimensão zero (ponto quântico), isto é, que confina os elétrons nas três direções espaciais [2]. Existem diversas formas de se produzir um ponto quântico. Por exemplo, através de técnicas litográficas pode-se depositar eletrodos metálicos sobre uma superfície semicondutora sob a qual reside um gás de elétrons bidimensional ${ }^{1}$. Ao se aplicar tensões negativas nesses contatos, o gás de elétrons se depleta, deixando uma região vazia de portadores que corresponde ao ponto quântico [3]. O tamanho típico de um ponto quântico varia de alguns nanometros até centenas de nanometros, e o seu tamanho, formato e interações podem se controladas/manipuladas através das técnicas avançadas de nanofabricação.

Devido ao confinamento eletrônico, surgem níveis de energia no ponto quântico semelhantes aos de um átomo ${ }^{2}$, com a vantagem de ser possível ajusta-los em laboratório. Assim é possível gerar uma "tabela periódica" completa para pontos quânticos, variando simplesmente uma tensão de porta [2]. A figura (1.1) ilustra

\footnotetext{
${ }^{1}$ Esse gás bidimensional reside, por exemplo, na interface entre as camadas de GaAs e AlGaAs de uma heteroestrutura.

${ }^{2}$ Ressaltamos que não estamos tratando aqui de pontos metálicos, onde se tem um contínuo de energia. Os pontos quânticos de nosso interesse apresentam níveis de energia discretos.
} 
uma geometria utilizada para transporte elétrico em pontos quânticos. Temos uma fonte de elétrons (reservatório emissor) à esquerda do ponto quântico e um reservatório coletor à direita, ou o contrário dependendo do sinal da tensão $V$. Os terminais estão espaçados do ponto quântico por barreiras de tunelamento. As dimensões e a energia eletrostática do ponto quântico podem ser ajustadas aplicando-se uma tensão de porta $V_{g}$ através de um terceiro terminal que não participa diretamente da transferência de carga. A descrição do transporte elétrico nesse sistema pode ser feita mediante técnicas de transporte quântico, conforme mencionamos na próxima seção.

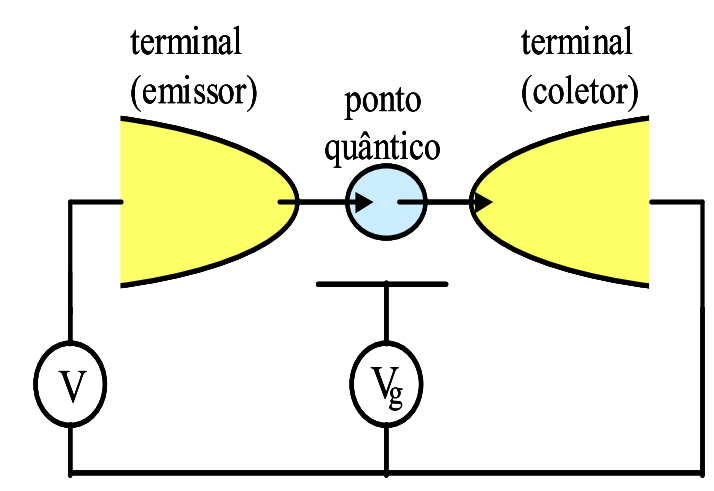

Figura 1.1: Ilustração esquemática de um ponto quântico acoplado a eletrodos. Os eletrodos da esquerda e da direita funcionam como emissor e coletor de elétrons, respectivamente. Um terceiro eletrodo se acopla capacitivamente ao ponto quântico, permitindo variar a sua energia eletrostática através da tensão de porta $V_{g}$. Com isso pode-se mudar a posição dos níveis de energia do ponto quântico. Na presença de uma tensão $V$ há um desbalanço entre os potenciais químicos dos reservatórios, podendo levar a uma corrente de tunelamento.

\subsection{Transporte quântico}

Existem alguns formalismos para se estudar transporte quântico. Por exemplo, o formalismo de funções de Green de não-equilíbrio, o formalismo de LandauerBüttiker, equação mestra e equação de Boltzmann. Nesse trabalho, porém, focaremos nas funções de Green de não-equilíbrio, e por vezes faremos conexão com a abordagem de Landauer-Büttiker, visto que as funções de Green recuperam as equações de Landauer-Büttiker quando não há interações no sistema. A vantagem do formalismo de funções de Green está na possibilidade de se incluir interações de muitos corpos como elétron-elétron, elétron-fônon, ao contrário da abordagem de Landauer-Büttiker que é não interagente. 
O formalismo de funções de Green foi desenvolvido originalmente pelo físico russo Keldysh [4] em 1965 e independentemente por Kadanoff e Baym [5]. Porém, somente na década de 90, com a necessidade de se explicar experimentos, essa técnica começou a ser empregada para calcular propriedades de transporte (e.g., corrente, ruído) em sistemas nanoscópicos. Vale citar o trabalho pioneiro de Meir e Wingreen [6], no qual os autores apresentaram uma equação para corrente em um sistema constituído por dois terminais acoplados a uma região central, que pode ser, por exemplo, um ponto ou um poço quântico. Posteriormente, Jauho, Meir e Wingreen [7] sistematizaram a forma de se calcular corrente, uma vez dado o Hamiltoniano do sistema, e aplicaram suas equações para tratar perturbações independentes e dependentes do tempo.

Desde esses trabalhos originais, as funções de Green de não-equilíbrio vem sendo aplicadas para se estudar transporte através de pontos quânticos em diversos regimes. Por exemplo, no regime Kondo, onde se procura entender teoricamente os efeitos sobre a ressonância Kondo da tensão externa [8], do espalhamento de spin [9]-[11], de terminais ferromagnéticos [9],[12], etc. O efeito Kondo em pontos quânticos também vem sendo estudado experimentalmente. Por exemplo, se observou a dependência do efeito Kondo com a temperatura [13], com a ocupação do ponto quântico [14], e com a assimetria das barreiras de tunelamento na presença de uma tensão externa [15].

Um outro regime de particular interesse é o de bloqueio de Coulomb [16]. Quando um elétron entra no ponto quântico, ocorre um acréscimo $U$ na energia eletrostática do sistema. Se $U$ exceder a energia de Fermi do terminal emissor e a energia térmica $k_{B} T$, um segundo elétron será impedido (bloqueado) de tunelar para dentro do ponto quântico. A figura (1.2) ilustra esquematicamente o perfil de potencial de um ponto quântico acoplado a terminais no regime de bloqueio de Coulomb. Temos que $\mu_{L}$ e $\mu_{R}$ são os potenciais químicos da esquerda e da direita, respectivamente, $\epsilon_{0}$ é o nível do ponto quântico (que consideramos degenerado em spin), e $U$ a energia de adição de um segundo elétron. Na configuração ilustrada, muito embora haja uma tensão externa aplicada, não existirá corrente fluindo pelo sistema, visto que a energia de adição é maior do que $\mu_{L}$. Variando uma tensão de porta $V_{g}$ pode-se deslocar o canal $\epsilon_{0}+U$ para dentro da janela de condução do sistema, resultando assim numa corrente não nula. Para um ponto quântico com multiplos canais, um gráfico de $I$ versus $V_{g}$ resultaria numa curva com vários picos, se uma pequena tensão $V$ estivesse presente. Se, por exemplo, os níveis degenerados em spin forem $E_{1}, E_{2}, E_{3}, \ldots$, os vários picos apareceriam em $E_{1}$, $E_{1}+U, E_{2}+2 U, E_{2}+3 U, \ldots$ [17]. Esses vários picos na curva $I \times V_{g}$ podem ser vistos, por exemplo, em [2] e [18].

Existem vários trabalhos na literatura que discutem transporte no regime de bloqueio de Coulomb. Por exemplo, Barner e Ruggiero [19] analisaram os efeitos do bloqueio de Coulomb na curva característica $I-V$ de um arranjo de aglom- 
erados (clusters) metálicos (partículas de Ag) acoplados a eletrodos. Os autores observaram uma sucessão de degraus na curva $I-V$ espaçados pela energia de carregamento $e^{2} / C$ da ilha metálica, onde $C$ é a capacitância do sistema. Schelp et al. [20] mediram magneto-resistência em um sistema de eletrodos de Cobalto acoplados, via barreiras de tunelamento, a ilhas metálicas de Co, porém, não observaram nenhum efeito apreciável do bloqueio de Coulomb sobre a magneto-resistência. Lee et al. [18] observaram, em um sistema de ponto quântico, oscilações na corrente como função da tensão de porta, provocadas pelo bloqueio de Coulomb. Seguimos vendo como a eletrônica dependente de spin surge dentro desse contexto.

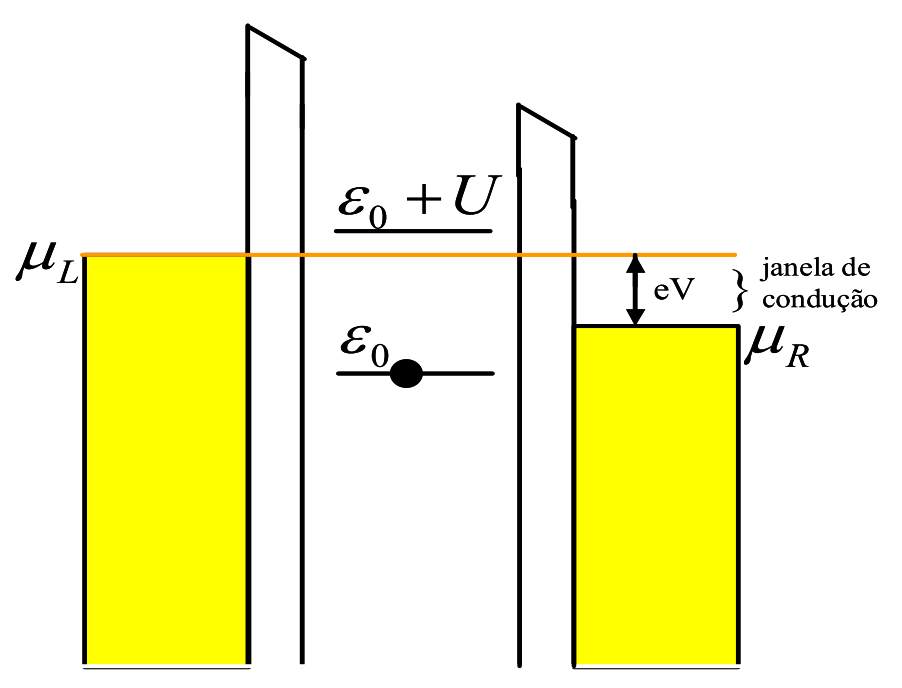

Figura 1.2: Perfil do potencial de um ponto quântico acoplado a terminais com potenciais químicos $\mu_{L}$ e $\mu_{R}$. Temos que $\epsilon_{0}$ é um nível do ponto quântico e $U$ a energia necessária para adicionar um segundo elétron, isto é, quando um elétron ocupa o estado $\epsilon_{0}$ um segundo elétron de spin oposto precisará de uma energia adicional $U$ para vencer a repulsão coulombiana e entrar no ponto quântico. Na configuração ilustrada, os elétrons do reservatório emissor têm energia abaixo de $\epsilon_{0}+U$ e portanto um segundo elétron estará energeticamente proibido de tunelar. Esse efeito recebe o nome de bloqueio de Coulomb. 


\subsection{Eletrônica de spin}

Uma abordagem recente sobre os sistemas nanoscópicos, sejam esses pontos quânticos, poços quânticos, super-redes, etc, consiste na manipulação e controle do spin dos portadores, visando a geração de novos efeitos e dispositivos. Essa área emergente denomina-se eletrônica de spin ou simplesmente "spintronica" [21]-[24].

Muito embora a eletrônica de spin tenha começado a receber intensa atenção a partir do final da década de 90, podemos dizer que sua origem em sistemas metálicos remonta à década de 70, com o trabalho de Julliere sobre magneto resistência de tunelamento (TMR) [25]. Julliere observou que a condutância da junção Fe-Ge-Co dependia fortemente do alinhamento magnético dos filmes de Fe e Co, sendo a condutância máxima quando a magnetizações dos filmes eram alinhadas paralelamente e mínima no caso de alinhamento antiparalelo. A variação observada da condutância foi de $14 \%$ à tensão nula e 4.2 K. Em 1995 Moodera et al. [26] melhoraram esse resultado obtendo uma variação de resistência da ordem de $11 \%$ a $295 \mathrm{~K}$ e de $24 \%$ a $4.2 \mathrm{~K}$ para uma junção de $\mathrm{CoFe} / \mathrm{Al}_{2} \mathrm{O}_{3} /$ Co. Além disso, esses valores permaneceram constantes até aproximadamente uma tensão de $100 \mathrm{mV}$, enquanto que no trabalho de Julliere havia uma supressão significativa da magnetoresistência para alguns $\mathrm{mV}$ aplicados através da junção. O efeito TMR é relevante para uma série de aplicações tecnológicas, como, por exemplo, sensores de campo magnético [27], leitores de disco rígido [28] e dispositivos para memória não-volátil [29].

O efeito TMR também pode ser observado em junções ferromagnéticas com uma ilha metálica ou um ponto quântico central. Resultados teóricos preveem que a competição entre os efeitos magnéticos e de acúmulo de carga podem levar a oscilações da TMR como função da tensão [30] e a um aumento da TMR no regime de bloqueio de Coulomb [31],[32]. Além disso, experimentos recentes de tunelamento por estados localizados, mostraram a possibilidade de se gerar magneto resistência de tunelamento negativas [33].

Em 1988 um outro efeito dependente de spin de grande impacto tecnológico foi descoberto por M. Baibich e colaboradores [34]. Estudando uma super-rede de $\mathrm{Fe} / \mathrm{Cr}$, observaram que a resistividade desse sistema a $4.2 \mathrm{~K}$ poderia diminuir de um fator da ordem de 2, quando as camadas ferromagnéticas adjacentes passavam do alinhamento antiparalelo para paralelo. Esse efeito, denominado de magneto

resistência gigante (GMR), é a base dos leitores de disco rígido dos computadores atuais.

Atualmente a eletrônica de spin tem avançado no sentido da nanoeletrônica, isto é, dispositivos baseados em nano-estruturas como pontos e poços quânticos. Podemos citar, por exemplo, a proposta de um transistor de efeito de campo e dependente de spin (spin-FET) [35]. Nesse sistema tem-se um canal bidimensional de alta mobilidade acoplado a terminais ferromagnéticos, sendo um terminal emis- 
sor e outro coletor de elétrons. Sobre esse canal aplica-se uma tensão de porta $V_{g}$ que gera um campo elétrico no interior do canal. O terminal emissor fornece elétrons com spins alinhados ao longo da sua direção de magnetização, enquanto o coletor opera como um filtro de spin, deixando entrar somente os elétrons que preservam o seu spin ao atravessar o sistema. Na ausência de espalhamento de spin no canal condutor, todo elétron que sai do emissor entra no coletor. Porém, o campo elétrico aplicado perpendicularmente ao canal bidimensional, modula a intensidade de uma interação spin-órbita (efeito Rashba) que tende a girar os spins eletrônicos de sua orientação inicial (mesma orientação do terminal emissor). Esse efeito resulta numa supressão da corrente. Com isso, é possível controlar a corrente através da tensão de porta $V_{g}$. Realizações experimentais desse dispositivo vem sendo testadas atualmente por vários grupos no exterior [36].

Um outro efeito interessante em junções ferromagnéticas é a possibilidade de se controlar a polarização da corrente $\wp$ através da tensão de porta $V_{g}$ ou da tensão entre os eletrodos $V$. Para uma junção do tipo ferromagneto-metalnormal-ferromagneto (FM-NM-FM), Kuo e Chen [37] mostraram que os eletrodos ferromagnéticos, quando alinhados antiparalelamente, levam a um desbalanço de população de spins na região central (metal normal). Esse desbalanço cria uma dependência da polarização da corrente como função da tensão de porta.

Vale ainda citar outras propostas de dispositivos spintrônicos como filtros de spin [38], o transistor de spin de Johnson [39], filtro e memória de spin em pontos quânticos [40], e geração de qubits (bits quânticos) em pontos quânticos [41], para uma possível implementação da computação quântica [42].

\subsection{Ruído}

Uma outra grandeza relevante no estudo do transporte em sistemas mesoscópicos é o ruído, pois fornece informações adicionais sobre um dado sistema, que não estão contidas na corrente média. Por exemplo, medidas de shot noise em contatos pontuas quânticos (quantum point contact) no regime Hall fracionário, revelaram a existência de quase-partículas de carga e/3 [43]. Outras aplicações do shot noise ainda podem ser vistas em [44].

O ruído de uma maneira geral são variações temporais aleatórias de uma determinada grandeza em torno do seu valor médio. Mais especificamente em condutores elétricos, há varias fontes de ruído. Por exemplo, o ruído $1 / f$, que em geral se observa em junções de tunelamento a baixas freqüências, o ruido térmico, que está relacionado ao movimento térmico aleatório dos portadores de carga, e o shot noise, que se deve a natureza discreta da carga elétrica [45]. Em outras palavras, a corrente elétrica é resultado da passagem de cargas discretas (elétrons) pelo sistema, essa granulosidade do fluxo de portadores gera o shot noise. 
Matematicamente o ruído é definido como a função auto-correlação correntecorrente,

$$
S\left(t, t^{\prime}\right)=\left\langle\left\{\delta I(t), \delta I\left(t^{\prime}\right)\right\}\right\rangle,
$$

onde $\delta I(t)=I(t)-\langle I\rangle$, sendo $I(t)$ a corrente no instante $t$, e $\langle I\rangle$ o seu valor médio. Essa definição inclui o shot noise e o ruído térmico. Vale salientar que o ruído térmico pode ocorrer mesmo quando não há uma corrente fluindo pelo sistema (tensão nula), isto é, trata-se de uma flutuação de equilíbrio, ao contrário do shot noise que é uma flutuação de não equilíbrio [45].

Quando a transferência de elétrons através de um condutor é descrita pela estatística de Poisson, que é utilizada para analisar eventos que não estão correlacionados no tempo, o shot noise é dado pelo valor de Poisson $S=2 e\langle I\rangle$. Porém, quando correlações estão presentes, $S$ desvia-se do valor poissônico $2 e\langle I\rangle$, podendo ser suprimido ou intensificado, dependendo do tipo de correlação. Para "medir" o desvio do shot-noise com relação ao seu valor clássico (poissônico), define-se o fator de Fano $\gamma=S / 2 e\langle I\rangle$. Quando $\gamma$ se desvia de 1, significa que há algum tipo de correlação no sistema (e.g. Pauli, Coulomb).

A supressão do ruído $(\gamma<1)$ em sistemas de dupla barreira com tunelamento ressonante (poço quântico acoplado a terminais) foi observada originalmente por Li et al. [46], e posteriormente por Liu et al. [47], onde constataram que $\gamma \approx 0.5$ para barreiras simétricas, e $\gamma \approx 1$ para barreiras assimétricas. A supressão do ruído no caso simétrico deve-se ao princípio de Pauli. Mais especificamente, um elétron ao tunelar para dentro da região entre as barreiras, ocupando assim um $i$-ésimo nível do poço, permanecerá aí por algum tempo antes de sair para o terminal coletor. Durante o seu tempo de permanência, um segundo elétron de mesmo spin fica proibido de ocupar esse estado. Logo, há uma correlação no transporte eletrônico, que tende a atrasar duas transições sucessivas pelo sistema. Isso resulta numa supressão do ruído $(\gamma \approx 0.5)$. No caso fortemente assimétrico o transporte é governado essencialmente pela barreira maior, o que resulta numa transmissão mais descorrelacionada $(\gamma \approx 1)$.

Ao contrário do princípio de Pauli, que sempre suprime o ruído, a interação de Coulomb pode aumentá-lo ou suprimi-lo. Por exemplo, observou-se $\gamma \approx 6.6$ para um sistema de poço quântico com tunelamento ressonante [48], em que um elétron ao tunelar do emissor para o poço modifica a energia eletrostática do sistema, levando a uma maior ressonância dos estados do poço com os estados do emissor. Com isso a transição emissor $\rightarrow$ poço é favorecida, resultando num aumento do shot-noise $(\gamma>1)$. Também se observou um aumento do shot noise $(\gamma>1)$ em sistema de tunelamento ressonante via estados localizados [49]. Atribui-se esse efeito a correlações de Coulomb entre elétrons que tunelam através de dois estados localizados vizinhos.

Por outro lado, a interação de Coulomb também pode suprimir o ruído. Por 
exemplo, Anda e Latgé [50] mostraram que o ruído, em um sistema de ponto quântico acoplado a terminais, apresenta uma supressão no regime de bloqueio de Coulomb. Birk et al. [51] mediram ruído no regime de bloqueio de Coulomb em um sistema constituído de uma nano-partícula entre uma ponta de STM (scanning tunneling microscopy) e uma superfície metálica. Os autores observaram uma supressão do ruído devido a efeitos de carregamento da nano-partícula.

Mais recentemente, Nauen et al. [52] estudando transporte em pontos quânticos de InAs auto-organizados, observaram uma sucessão de picos no fator de Fano, como função da tensão externa. Esses picos são provenientes das sucessivas ressonância dos níveis dos pontos quânticos como função da tensão $V$. Posteriormente, Nauen et al. [53] analisaram o shot noise através de um único ponto quântico de InAs, onde encontraram uma dependência linear do fator de Fano como função da tensão entre os eletrodos.

Atualmente o ruído vem sendo estudado teoricamente também em dispositivos ferromagnéticos. Bulka et al. [54] estudaram pela primeira vez ruído numa junção metálica do tipo FM-NM-FM. Os autores observaram que o acúmulo de spin no metal normal, devido aos terminais ferromagnéticos, dá origem a uma flutuação de spin que, por sua vez, amplifica o ruído. Posteriormente, Tserkovnyak e Brataas [55] analisaram a dependência do ruído como função do ângulo $\theta$ entre os alinhamentos magnéticos das camadas ferromagnéticas da junção FM-NM-FM, observando um comportamento não monotônico do shot-noise como função de $\theta$. Além disso, Lopez e Sanchez [56], através do cálculo do shot noise, encontraram que a temperatura Kondo em um sistema de ponto quântico acoplado a terminais ferromagnéticos, pode ser suprimida como função da polarização dos terminais, quando esses terminais tem suas magnetizações alinhadas paralelamente.

\subsection{Nossos resultados}

O nosso trabalho nasce dentro do contexto da eletrônica de spin. O primeiro passo de nosso estudo foi a obtenção de equações que descrevem corrente e ruído dependentes de spin, utilizando a técnica de funções de Green de não-equilíbrio [57]. Apresentamos uma maneira sistemática de se derivar a equação do ruído (térmico+shot noise), definindo funções de Green de quatro operadores, e efetuando expansões da matriz $S^{3}$. Por vezes, fizemos conexão entre nossas expressões e as fórmulas de Landauer-Büttiker.

Com o formalismo desenvolvido, analisamos três sistemas: (I) uma junção do tipo ferromagneto-ponto-quântico-ferromagneto (FM-PQ-FM), (II) um ponto quântico acoplado a múltiplos terminais ferromagnéticos e (III) um poço quântico

\footnotetext{
${ }^{3}$ A matriz $S$ no formalismo de funções de Green é responsável pela mudança de representação dos operadores de campo da representação de Heisenberg para a representação de interação.
} 
acoplado a terminais DMS (dilute magnetic semiconductors). Resumimos a seguir as nossas principais contribuições em cada um desses sistemas, assim com as fórmulas deduzidas aqui para descrever o transporte quântico dependente de spin.

No capítulo 3 derivamos fórmulas para corrente e ruído (térmico e shot noise), utilizando o formalismo de funções de Green de não-equilíbrio. Em particular para o sistema FM-PQ-FM, as nossas expressões para a corrente e para o ruído levam em conta a polarização dos terminais, a interação de Coulomb e espalhamento de spin no ponto quântico [58],[59]. Também determinamos uma fórmula para o ruído no sistema de um poço quântico acoplado a contatos DMS, que leva em conta os efeitos dos terminais DMS e da quantização de Landau na presença de campo magnético.

Para o sistema I (capítulo 4), encontramos de maneira auto-consistente as ocupações de elétrons no ponto quântico, a sua densidade de estados, a corrente e o ruído. Analisamos essas grandezas nos alinhamentos paralelo $(\mathrm{P})$ e anti-paralelo (AP) dos eletrodos ferromagnéticos. Encontramos um desbalanço das populações de spin (spin imbalance) no ponto quântico devido aos terminais ferromagnéticos em ambas as configurações magnéticas P e AP. Esse efeito, combinado com a interação de Coulomb leva a um bloqueio de Coulomb dependente de spin, e conseqüentemente a uma polarização $\wp$ da corrente que varia com a tensão externa $V$. Vale salientar que $\wp$ apresenta comportamentos contrastantes entre os caso P e AP: na configuração P a polarização é suprimida numa certa faixa de tensão, enquanto no caso AP ela é intensificada nessa mesma faixa, ou o contrário, dependendo do sinal da tensão externa $V$.

Também observamos que as populações de spin no ponto quântico dependem do sinal (direto/reverso) da tensão aplicada. Por exemplo no caso AP, para tensões positivas (direta) temos $n_{\uparrow}>n_{\downarrow}$, enquanto que para tensões negativas (reversa) ocorre o contrário, isto é, $n_{\uparrow}<n_{\downarrow}$. Essa inversão de população como função do sinal de $V$, leva também a uma inversão da polarização da corrente como função do sinal da tensão, devido a um bloqueio de Coulomb dependente de spin. Também mostramos que a magnetização $m$ do ponto quântico pode ser controlada em módulo e sentido através da voltagem. Além disso, mostramos ser possível trocar o sinal de $m$ mudando o alinhamento magnético dos terminais de $\mathrm{P}$ para AP.

Ainda, encontramos que a interação de Coulomb pode intensificar a magneto resistência de tunelamento (TMR), dentro do regime de bloqueio de Coulomb, enquanto o espalhamento de spin tende a suprimir o sinal de TMR [60]. Notamos também que os efeitos combinados da interação de Coulomb e do espalhamento de spin podem resultar numa supressão adicional do TMR.

Estudamos os efeitos das polarizações dos eletrodos ferromagnéticos, do espalhamento de spin e da interação de Coulomb sobre o shot noise. Observamos uma dependência não monotônica do shot noise como função da taxa de espalhamento 
de spin, ao contrário da corrente que se comporta monotônicamente como função dessa taxa. Encontramos que o fator de Fano $\gamma$ é suprimido na configuração AP devido a espalhamento de spin, e que essa supressão intensifica-se com o aumento da polarização dos eletrodos, chegando a $\gamma \approx 0.2$ quando os terminais são $100 \%$ polarizados. Também mostramos, através do fator de Fano, uma analogia entre o sistema FM-PQ-FM com espalhamento de spin e o problema não magnético de tripla-barreira de tunelamento. Por último, comparamos os efeitos do espalhamento de spin sobre o ruído, nos casos de barreiras de tunelamento simétricas e fortemente assimétricas.

Sistema II (capítulo 5). Aqui investigamos um sistema constituído por um ponto quântico acoplado, via barreiras de tunelamento, a múltiplos terminais ferromagnéticos. Formulamos o problema para um número qualquer de eletrodos, e em seguida particularizamos para o caso de três terminais ferromagnéticos. Um terminal funciona como emissor de elétrons, enquanto os outros dois como reservatórios coletores. Mostramos que esse sistema permite gerar simultaneamente polarizações $\uparrow$ e $\downarrow$ de corrente em terminais diferentes. Por exemplo, a corrente pode sair $\uparrow$-polarizada do terminal emissor e entrar $\downarrow$-polarizada em um dos terminais coletores. Além disso, pode haver uma amplificação da polarização da corrente ao passar de um terminal a outro.

Sistema III (capítulo 6). Por último estudamos um sistema composto por um poço quântico acoplado, via barreiras de tunelamento, a terminais de semicondutor magnético diluído (DMS) ${ }^{4}$. O caso de terminais não-magnéticos (NMS) também foi abordado para efeito de comparação. Na presença de um campo magnético externo, a interação de troca $s$ - $d$ entre os elétrons itinerantes e os elétrons localizados dos átomos magnéticos (e.g. Mn), leva a um desdobramento das bandas para spin $\uparrow$ e $\downarrow$, resultando numa magnetização efetiva dos terminais DMS. Os efeitos combinados dessa magnetização e dos níveis de Landau dentro do poço, fazem as correntes $\uparrow$ e $\downarrow$, e os seus respectivos ruídos, diferirem significativamente, gerando polarizações dependentes da tensão entre os terminais. Além disso encontramos oscilações na corrente, no ruído e no fator de Fano como função do campo magnético. Observamos que o fator de Fano apresenta picos sempre que um nível de Landau alinha-se ao potencial químico do reservatório emissor. Por último, fazemos uma conexão da nossa abordagem com o formalismo de Landauer-Büttiker, onde encontramos uma versão resolvida em spin das equação de Landauer-Büttiker.

\footnotetext{
${ }^{4}$ Filtragem de spin e magnetoresistência em sistemas de uma e duas barreira com contatos e/ou poço DMS foram estudados no regime coerente por J. C. Egues et al. [61], e recentemente A. Slobodskyy et al. [62] investigaram transporte spin polarizado em um sistema de dupla barreira com material DMS no poço.
} 


\section{Capítulo 2}

\section{Introdução às Funções de Green de Não-Equilíbrio}

O problema proposto pela teoria de transporte, seja esse quântico ou clássico, consiste no cálculo dos efeitos induzidos em um dado sistema por um campo externo (e.g. campo elétrico, fotoexcitação, etc.). Esses efeitos podem ser, por exemplo, a corrente de portadores de carga (elétrons e buracos) em uma superfície semicondutora excitada por pulsos ultra-curtos [66], a corrente de tunelamento e o seu ruído (shot-noise) em sistemas de pontos quânticos [6], poços quânticos [67], heteroestrutras, etc, na presença de uma tensão externa, dentre outros.

As funções de Green de não equilíbrio constituem uma ferramenta matemática utilizada para se estudar transporte quântico. Essa técnica foi originalmente proposto por L. V. Keldysh [4] e independentemente por L. Kadanoff e G. Baym [5]. Desde esses trabalhos originais, esse formalismo vem sendo cada vez mais aplicado em sistemas fora do equilíbrio. Atualmente as funções de Green de não-equilíbrio constituem uma das principais técnicas para se estudar transporte em sistemas de baixa dimensionalidade como pontos e poços quânticos [68].

Aqui apresentamos algumas noções básicas sobre as funções de Green de não equilíbrio, que serão aplicadas no decorrer do nosso estudo.

\subsection{Formulação do problema}

Formula-se o problema quântico de não-equilíbrio considerando o Hamiltoniano $H=h+H^{\prime}(t)$, onde $h$ é o Hamiltoniano do sistema independente do tempo, que contém termos de partícula livre $H_{0}$ e interações de muitos corpos $H^{i}$, como a interação elétron-elétron. Dizemos que $h$ é o termo de equilíbrio pois esse termo não gera nenhum fluxo de carga ou energia, o que caracterizaria uma situação de não-equilíbrio. O termo $H^{\prime}(t)$, por outro lado, é responsável por tirar o sistema do equilíbrio, podendo representar um campo elétrico, uma excitação por luz, um acoplamento entre contatos com diferentes potenciais químicos, etc. Em particular, 
em nosso trabalho esse termo gera uma corrente elétrica no sistema.

Assumimos que o termo de não-equilíbrio é ligado no instante $t_{0}$, e portanto para $t<t_{0}$ temos $H^{\prime}=0$ e o Hamiltoniano total do sistema é simplesmente $H=h$. Em geral, quando se está interessado em regime estacionário adota-se $t_{0} \rightarrow-\infty$ [57]. Para tempos menores do que $t_{0}$ temos que o sistema está em equilíbrio térmico, visto que $H^{\prime}=0$, a uma temperatura dada por $\beta$, onde $\beta=1 / k_{B} T$. Ressaltamos que muito embora tenhamos usado $T$ para definir $\beta$ como o inverso da temperatura, o símbolo $T$ não será usado no decorrer desse capítulo para expressar temperatura. Esse símbolo representará o operador ordenamento temporal.

A matriz densidade de equilíbrio é dada por

$$
\rho_{0}=\frac{e^{-\beta h}}{\operatorname{Tr} e^{-\beta h}},
$$

onde o símbolo Tr denota traço e é a soma sobre alguma base completa de estados:

$$
\operatorname{Tr} \equiv \sum_{i}<i, t_{0}|\cdots| i, t_{0}>
$$

Em equilíbrio o valor esperado $\langle O\rangle$ de um dado observável físico $O$ é simplesmente o traço desse operador na representação de Schrödinger pesado por $\rho_{0}$,

$$
<O>=\operatorname{Tr}\left\{\rho_{0} O\right\}
$$

A principal tarefa no formalismo de não equilíbrio consiste no calculo do valor esperado $\langle O\rangle$ após "ligarmos" o termo de não equilíbrio do Hamiltoniano, isto é, tempos $t>t_{0}$. Existem algumas propostas na literatura para se calcular essa média. Por exemplo, Gerald D. Mahan [70] propõe uma matriz densidade $\rho$ que inclui o termo de não equilíbrio $H^{\prime}$ e tem a forma $\rho(t)=\rho_{0}+f(t)$, onde $\rho_{0}$ é a matriz densidade independente do tempo (de equilíbrio), e $f(t)$ contém os efeitos de não equilíbrio. Através da equação de Heisenberg,

$$
\frac{\partial \rho(t)}{\partial t}=\frac{\partial f(t)}{\partial t}=-i\left[h+H^{\prime}, \rho(t)\right]
$$

procura-se encontrar uma expressão para $f(t)$ e com isso calcular o valor esperado de observáveis. Uma outra definição de média de não-equilíbrio foi proposta por Kadanoff e Baym em seu trabalho pioneiro sobre funções de Green aplicadas a problemas de não-equilíbrio [5]. Os autores definem média de não equilíbrio da seguinte forma

$$
<O>=\operatorname{Tr}\left\{\rho_{0} O_{H}\right\}
$$

onde $O_{H}$ é o operador $O$ na representação de Heisenberg, e portanto evolui segundo a equação

$$
\frac{\partial O_{H}(t)}{\partial t}=i\left[H, O_{H}(t)\right]
$$

Note que na média (2.5) utiliza-se a matriz densidade de equilíbrio, e não um $\rho(t)$ como propõe Mahan [70]. Em outras palavras, calcula-se a média de um operador 
de não-equilíbrio $O_{H}$ sobre um ensemble que preserva suas características originais $\left(t<t_{0}\right)$. A. P. Jauho e H. Haug dão um argumento físico simples para o ansatz (2.5) dizendo que os graus de liberdade termodinâmicos contidos em $h$ não seguem instantaneamente a variação contida em $H^{\prime}$ [57]. Neste trabalho adotaremos a definição de média (2.5).

Quando se deseja calcular o valor esperado de produtos de operadores com tempos diferentes, $<O(t) O^{\prime}\left(t^{\prime}\right)>$ recorre-se às funções de Green, conforme discutimos a seguir.

\subsection{A função de Green ordenada no contorno}

No formalismo de equilíbrio costuma-se definir a função de Green causal

$$
G^{t}\left(t_{1}, t_{1}^{\prime}\right)=-i\left\langle T \psi_{H}\left(t_{1}\right) \psi_{H}^{\dagger}\left(t_{1}^{\prime}\right)\right\rangle
$$

onde $\psi_{H}$ e $\psi_{H}^{\dagger}$ são operadores fermiônicos na representação de Heisenberg ${ }^{1}$, e $T$ o operador ordenamento temporal, que está definido abaixo

$$
T \psi_{H}\left(t_{1}\right) \psi_{H}^{\dagger}\left(t_{1}^{\prime}\right) \equiv \begin{cases}\psi_{H}\left(t_{1}\right) \psi_{H}^{\dagger}\left(t_{1}^{\prime}\right) & t_{1}>t_{1}^{\prime} \\ -\psi_{H}^{\dagger}\left(t_{1}^{\prime}\right) \psi_{H}\left(t_{1}\right) & t_{1}<t_{1}^{\prime}\end{cases}
$$

Da equação (2.8) vemos que $T$ move o operador de maior tempo para a esquerda (em inglês existe uma regra mnemônica para esse ordenamento: "late goes to left"). A relevância da função de Green causal na teoria de equilíbiro deve-se à existência de uma expansão diagramática para $G^{t}[70]$.

No formalismo de não equilíbrio a quantidade que também possui uma expansão perturbativa diagramática é a função de Green ordenada no contorno ${ }^{2}$. Essa função de Green torna a teoria de não equilíbrio estruturalmente idêntica à teoria de equilíbrio [71]. Essa função de Green é definida da seguinte forma

$$
G\left(t_{1}, t_{1}^{\prime}\right)=-i\left\langle T_{C} \psi_{H}\left(t_{1}\right) \psi_{H}^{\dagger}\left(t_{1}^{\prime}\right)\right\rangle,
$$

onde o contorno $C$ inicia e termina em $t_{0}$, caminha ao longo do eixo real e passa por $t_{1}$ e $t_{1}^{\prime}$ somente uma vez [Fig.2.1(a)]. Em geral adota-se $t_{0} \rightarrow-\infty$. Assim o contorno começa em $-\infty$, passa pelos pontos $t_{1}$ e $t_{1}^{\prime}$, e retorna a $-\infty$. O operador ordenamento temporal $T_{C}$ ordena os operadores da seguinte forma

$$
T_{C} \psi_{H}\left(t_{1}\right) \psi_{H}^{\dagger}\left(t_{1}^{\prime}\right) \equiv \begin{cases}\psi_{H}\left(t_{1}\right) \psi_{H}^{\dagger}\left(t_{1}^{\prime}\right) & t_{1}>_{c} t_{1}^{\prime}, \\ -\psi_{H}^{\dagger}\left(t_{1}^{\prime}\right) \psi_{H}\left(t_{1}\right) & t_{1}<_{c} t_{1}^{\prime} .\end{cases}
$$

As desigualdades $t_{1}>_{c} t_{1}^{\prime}$ e $t_{1}<_{c} t_{1}^{\prime}$ indicam maior e menor ao longo do contorno. Portanto, o ordenamento ao longo de $C$ significa que os pontos no ramo que volta

\footnotetext{
${ }^{1}$ A mesma definição se aplica a operadores bosônicos.

${ }^{2}$ Essa função foi originalmente denominada por Schwinger de closed time path Green's function [69]
} 
para $-\infty$ correspondem a tempos maiores do que os pontos no ramo direto (que vem de $-\infty)$. Além disso entre dois pontos no ramo de retorno, o de maior tempo é o mais próximo de $-\infty$.

(a)

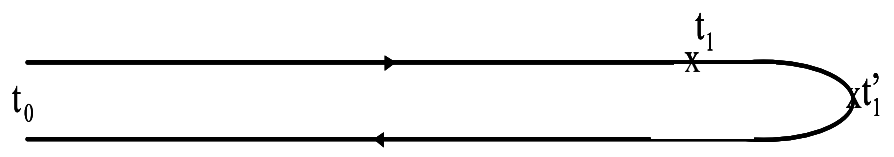

(b)

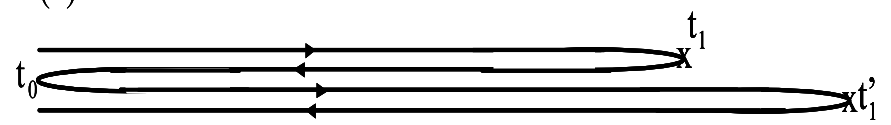

Figura 2.1: (a) Contorno de tempo utilizado na definição da função de Green ordenada no contorno, e (b) contorno deformado para se obter a continuação analítica. O contorno inicia em $t_{0}$, passa por $t_{1}$ e $t_{1}^{\prime}$, e retorna a $t_{0}$. Em geral toma-se $t_{0} \rightarrow-\infty$.

\subsection{A origem do contorno de integração}

Para se obter uma expansão diagramática da função de Green ordenada no contorno, devemos escrevê-la de tal forma que o teorema de Wick possa ser aplicado. O primeiro passo consiste em passar os operadores da representação de Heisenberg para a representação de interação com relação a $h$. Seja $O_{H}$ e $O_{h}$ o operador $O$ na representação de Heisenberg e de interação, respectivamente. Essas duas representações estão relacionadas por

$$
O_{H}=f_{h}^{\dagger}\left(t, t_{0}\right) O_{h}(t) f_{h}\left(t, t_{0}\right)=f_{h}\left(t_{0}, t\right) O_{h}(t) f_{h}\left(t, t_{0}\right)
$$

onde $f_{h}\left(t, t_{0}\right)=T e^{-i \int_{t_{0}}^{t} d t^{\prime} H_{h}^{\prime}\left(t^{\prime}\right)}$, e $H_{h}^{\prime}$ está na representação de interação, isto é, sua evolução temporal é dada por $\frac{\partial O_{h}}{\partial t}=i\left[h, O_{h}(t)\right]$. Note que a equação (2.11) descreve um "contorno": o tempo inicia em $t_{0}$, passa por $t$ e volta a $t_{0}$. Utilizando essa transformação na função de Green (2.9) vem

$$
G\left(1,1^{\prime}\right)=-i\left\langle T_{C} f\left(t_{0}, t\right) \psi_{h}(t) f\left(t, t^{\prime}\right) \psi_{h}\left(t^{\prime}\right) f\left(t^{\prime}, t_{0}\right)\right\rangle
$$

Em geral se toma $t_{0} \rightarrow-\infty$ de modo que a equação acima fica

$$
G\left(1,1^{\prime}\right)=-i\left\langle T_{C} f(-\infty, t) \psi_{h}(t) f\left(t, t^{\prime}\right) \psi_{h}\left(t^{\prime}\right) f\left(t^{\prime},-\infty\right)\right\rangle
$$


Como usamos desde o início o operador $T_{C}$ podemos reescrever a equação acima da seguinte forma

$$
G\left(1,1^{\prime}\right)=-i\left\langle T_{C} f_{C}(-\infty,-\infty) \psi_{h}(t) \psi_{h}\left(t^{\prime}\right)\right\rangle
$$

onde $f_{C}(-\infty,-\infty)$ contém operadores que atuam nos três intervalos de tempo $(-\infty, t),\left(t, t^{\prime}\right)$ e $\left(t^{\prime},-\infty\right)$. O operador $T_{C}$ automaticamente distribui esses operadores de tal forma que eles atuem na seqüência correta, dada pela Eq.(2.13).

Definindo $S_{C}=f_{c}(-\infty,-\infty)=e^{-i \int_{C} d \tau H_{h}^{\prime}(\tau)}$ podemos escrever

$$
G\left(1,1^{\prime}\right)=-i\left\langle T_{C} S_{C} \psi_{h}(1) \psi_{h}^{\dagger}\left(1^{\prime}\right)\right\rangle
$$

A expansão da matriz $S_{C}$ dá origem à diagramática no formalismo de não-equilíbrio, semelhantemente ao problema de equilíbrio $[70]^{3}$. Observe que se utilizássemos o operador ordenamento temporal $T$, ao invés de $T_{C}$, não seria correto escrever $G\left(1,1^{\prime}\right)=-i\left\langle T f_{C}(-\infty,-\infty) \psi_{h}(t) \psi_{h}\left(t^{\prime}\right)\right\rangle=-i\left\langle T S_{C} \psi_{h}(t) \psi_{h}\left(t^{\prime}\right)\right\rangle$, pois o operador $T$ faria um ordenamento cronológico e não ao longo do contorno $C$, e conseqüentemente a distribuição de operadores dado pela Eq.(2.13) não seria recuperada. O contorno $C$ então nada mais é do que uma forma de se definir adequadamente o ordenamento dos operadores na Eq.(2.15). Sem esse ordenamento $T_{C}$, não seria possível expressar a função de Green em termos da matriz $S$, como na Eq.(2.15).

\subsection{As funções de Green $G^{t}, G^{\widetilde{t}}, G^{<}$e $G^{>}$}

Podemos definir quatro funções de Green de acordo com a posição dos tempos $t_{1} \mathrm{e}$ $t_{1}^{\prime}$ ao longo do contorno. Denotando o ramo superior do contorno por $C_{1}$ e o ramo inferior por $C_{2}$, definimos as seguintes funções de Green de acordo com a posição dos tempos $t_{1}$ e $t_{1}^{\prime}$ ao longo do contorno,

$$
G\left(t_{1}, t_{1}^{\prime}\right)= \begin{cases}G^{t}\left(t_{1}, t_{1}^{\prime}\right)=-i\left\langle T \psi_{H}\left(t_{1}\right) \psi_{H}^{\dagger}\left(t_{1}^{\prime}\right)\right\rangle & t_{1}, t_{1}^{\prime} \in C_{1}, \\ G^{>}\left(t_{1}, t_{1}^{\prime}\right)=-i\left\langle\psi_{H}\left(t_{1}\right) \psi_{H}^{\dagger}\left(t_{1}^{\prime}\right)\right\rangle & t_{1} \in C_{2}, t_{1}^{\prime} \in C_{1}, \\ G^{<}\left(t_{1}, t_{1}^{\prime}\right)=i\left\langle\psi_{H}^{\dagger}\left(t_{1}^{\prime}\right) \psi_{H}\left(t_{1}\right)\right\rangle & t_{1} \in C_{1}, t_{1}^{\prime} \in C_{2}, \\ G^{\widetilde{t}}\left(t_{1}, t_{1}^{\prime}\right)=-i\left\langle\widetilde{T} \psi_{H}\left(t_{1}\right) \psi_{H}^{\dagger}\left(t_{1}^{\prime}\right)\right\rangle & t_{1}, t_{1}^{\prime} \in C_{2} .\end{cases}
$$

Temos que $\widetilde{T}$ é o operador anti-ordenamento temporal. Sua atuação nos operadores gera o seguinte ordenamento

$$
\widetilde{T} \psi_{H}\left(t_{1}\right) \psi_{H}^{\dagger}\left(t_{1}^{\prime}\right) \equiv \begin{cases}\psi_{H}\left(t_{1}\right) \psi_{H}^{\dagger}\left(t_{1}^{\prime}\right) & t_{1}<t_{1}^{\prime} \\ -\psi_{H}^{\dagger}\left(t_{1}^{\prime}\right) \psi_{H}\left(t_{1}\right) & t_{1}>t_{1}^{\prime}\end{cases}
$$

Mais especificamente temos

$$
G^{t}\left(t_{1}, t_{1}^{\prime}\right)=-i \theta\left(t_{1}-t_{1}^{\prime}\right)\left\langle\psi_{H}\left(t_{1}\right) \psi_{H}^{\dagger}\left(t_{1}^{\prime}\right)\right\rangle+i \theta\left(t_{1}^{\prime}-t_{1}\right)\left\langle\psi_{H}^{\dagger}\left(t_{1}^{\prime}\right) \psi_{H}\left(t_{1}\right)\right\rangle
$$

\footnotetext{
${ }^{3}$ No caso em que há interação de muitos corpos em $h$, uma segunda mudança de representação se faz necessária, antes de se aplicar o teorema de Wick, conforme está discutido em [71].
} 


$$
G^{\widetilde{t}}\left(t_{1}, t_{1}^{\prime}\right)=-i \theta\left(t_{1}^{\prime}-t_{1}\right)\left\langle\psi_{H}\left(t_{1}\right) \psi_{H}^{\dagger}\left(t_{1}^{\prime}\right)\right\rangle+i \theta\left(t_{1}-t_{1}^{\prime}\right)\left\langle\psi_{H}^{\dagger}\left(t_{1}^{\prime}\right) \psi_{H}\left(t_{1}\right)\right\rangle
$$

O anti-ordenamento temporal $\widetilde{T}$ é necessário pois quando $t_{1}$ e $t_{1}^{\prime}$ estão no ramo inferior, ocorre uma inversão entre ordenamento cronológico e ordenamento no contorno. Considere por exemplo $t_{1}>t_{1}^{\prime}$, isto é, cronologicamente $t_{1}$ é maior do que $t_{1}^{\prime}$. Porém, se $t_{1}$ e $t_{1}^{\prime}$ estão no ramo inferior temos $t_{1}<_{c} t_{1}^{\prime}$, que é justamente o contrário do ordenamento cronológico.

Outras duas funções de Green de grande utilidade para cálculos de transporte são as funções de Green avançada (a) e retardada (r), que estão definidas abaixo,

$$
\begin{aligned}
G^{a}\left(t_{1}, t_{1}^{\prime}\right) & =i \theta\left(t_{1}^{\prime}-t_{1}\right)\left\langle\left\{\psi_{H}\left(t_{1}\right), \psi_{H}^{\dagger}\left(t_{1}^{\prime}\right)\right\}\right\rangle \\
& =\theta\left(t_{1}^{\prime}-t_{1}\right)\left[G^{<}\left(t_{1}, t_{1}^{\prime}\right)-G^{>}\left(t_{1}, t_{1}^{\prime}\right)\right]
\end{aligned}
$$

e

$$
\begin{aligned}
G^{r}\left(t_{1}, t_{1}^{\prime}\right) & =-i \theta\left(t_{1}-t_{1}^{\prime}\right)\left\langle\left\{\psi_{H}\left(t_{1}\right), \psi_{H}^{\dagger}\left(t_{1}^{\prime}\right)\right\}\right\rangle \\
& =\theta\left(t_{1}-t_{1}^{\prime}\right)\left[G^{>}\left(t_{1}, t_{1}^{\prime}\right)-G^{<}\left(t_{1}, t_{1}^{\prime}\right)\right]
\end{aligned}
$$

As chaves $\{$,$\} denotam anti-comutador. Note que G^{r}-G^{a}=G^{>}-G^{<}$. A vantagem de se trabalhar com $G^{r}$ e $G^{a}$ é que essas duas funções de Green satisfazem a equação de Dyson, quando uma auto-energia $\Sigma$ pode ser definida. Já a função $G^{<}$é dada pela equação de Keldysh, conforme veremos abaixo.

\subsection{Continuação Analítica}

A função de Green ordenada no contorno é uma ferramenta matemática para tornar o formalismo de não-equilíbrio estruturalmente idêntico ao de equilíbrio. Porém, para calcularmos quantidades físicas precisamos conhecer as funções correlação $G^{<}$ e $G^{>}$e as funções $G^{r}$ e $G^{a}$. Essas quatro funções de Green são obtidas a partir da função ordenada no contorno $G\left(1,1^{\prime}\right)$ via o procedimento denominado continuação analítica.

Admitindo-se que uma auto-energia $\Sigma$ pode ser definida, a função de Green ordenada no contorno obedece a equação de Dyson,

$$
G\left(\tau_{1}, \tau_{1}^{\prime}\right)=G^{0}\left(\tau_{1}, \tau_{1}^{\prime}\right)+\int_{C} d \tau_{2} \int_{C} d \tau_{3} G^{0}\left(\tau_{1}, \tau_{2}\right) \Sigma\left(\tau_{2}, \tau_{3}\right) G\left(\tau_{3}, \tau_{1}^{\prime}\right)
$$

onde utilizamos a letra grega $\tau_{i}$ para indicar que o tempo $t_{i}(i=1,2,3)$ está sujeito ao ordenamento temporal ao longo do contorno $C$, portanto dizemos que a integral é feita ao longo desse contorno. Após a continuação analítica esse tempo passará a ser denotado simplesmente por $t_{i}$, visto que as funções de Green assumirão uma das quatro formas apresentadas anteriormente, $G^{<}, G^{>}, G^{t}, G^{\widetilde{t}}$, que já possuem um 
ordenamento bem definido. Para convertermos integrais de contorno em integrais diretas ao longo do eixo real, seguimos o procedimento apresentado por David C. Langreth [72].

Na equação de Dyson encontramos produtos de funções de Green da forma

$$
D\left(\tau_{1}, \tau_{1}^{\prime}\right)=\int_{C} d \tau A\left(\tau_{1}, \tau\right) B\left(\tau, \tau_{1}^{\prime}\right)
$$

onde $A, B$ e $D$ são funções de Green quaisquer com ordenamento temporal dado por $T_{C}$. Para calcular (2.23) vamos considerar $t_{1}<_{c} t_{1}^{\prime}$, isto é, $t_{1}^{\prime}$ vem posteriormente a $t_{1}$ ao longo do contorno, conforme ilustramos na Fig.2.1(a). Isso corresponde a $D^{<}\left(t_{1}, t_{1}^{\prime}\right)$, como definido na Eq.(2.16). A Eq.(2.23) pode então ser expressa na seguinte forma

$$
\begin{gathered}
D^{<}\left(t_{1}, t_{1}^{\prime}\right)=\int_{-\infty}^{t_{1}} d \tau A\left(t_{1}, \tau\right) B\left(\tau, t_{1}^{\prime}\right)+\int_{t_{1}}^{t_{1}^{\prime}} d \tau A\left(t_{1}, \tau\right) B\left(\tau, t_{1}^{\prime}\right) \\
+\int_{t_{1}^{\prime}}^{-\infty} d \tau A\left(t_{1}, \tau\right) B\left(\tau, t_{1}^{\prime}\right) .
\end{gathered}
$$

Reescrevendo a integral $\int_{t_{1}}^{t_{1}^{\prime}}$ na forma $\int_{t_{1}}^{t_{1}^{\prime}}=\int_{t_{1}}^{-\infty}+\int_{-\infty}^{t_{1}^{\prime}}$, a equação acima torna-se

$$
\begin{aligned}
D^{<}\left(t_{1}, t_{1}^{\prime}\right)= & \int_{-\infty}^{t_{1}} d \tau A\left(t_{1}, \tau\right) B\left(\tau, t_{1}^{\prime}\right)+\int_{t_{1}}^{-\infty} d \tau A\left(t_{1}, \tau\right) B\left(\tau, t_{1}^{\prime}\right) \\
& +\int_{-\infty}^{t_{1}^{\prime}} d \tau A\left(t_{1}, \tau\right) B\left(\tau, t_{1}^{\prime}\right)+\int_{t_{1}^{\prime}}^{-\infty} d \tau A\left(t_{1}, \tau\right) B\left(\tau, t_{1}^{\prime}\right) .
\end{aligned}
$$

A Eq.(2.25) equivale ao contorno ilustrado na Fig.2.1(b). O procedimento de se deformar o contorno $C$ para efetuar a continuação analítica de um produto de funções de Green foi originalmente proposto por D. C. Langreth [72].

Aplicando as definições de função de Green menor e maior obtemos, por exemplo, para o primeiro termo $\int_{-\infty}^{t_{1}} d t A^{>}\left(t_{1}, t\right) B^{<}\left(t, t_{1}^{\prime}\right)$. Note que temos $A^{>}\left(t_{1}, t\right)$ e $B^{<}\left(t, t_{1}^{\prime}\right)$ pois a integral $\int_{-\infty}^{t_{1}}$ envolve tempos $t$ menores do que $t_{1}$ e $t_{1}^{\prime}$ no sentido do contorno. A expressão completa está apresentada abaixo

$$
\begin{aligned}
D^{<}\left(t_{1}, t_{1}^{\prime}\right) & =\int_{-\infty}^{t_{1}} d t A^{>}\left(t_{1}, t\right) B^{<}\left(t, t_{1}^{\prime}\right)+\int_{t_{1}}^{-\infty} d t A^{<}\left(t_{1}, t\right) B^{<}\left(t, t_{1}^{\prime}\right) \\
& +\int_{-\infty}^{t_{1}^{\prime}} d t A^{<}\left(t_{1}, t\right) B^{<}\left(t, t_{1}^{\prime}\right)+\int_{t_{1}^{\prime}}^{-\infty} d t A^{<}\left(t_{1}, t\right) B^{>}\left(t, t_{1}^{\prime}\right) .
\end{aligned}
$$

Nessa última equação não precisamos mais falar de um ordenamento ao longo do contorno pois as funções de Green menor e maior já possuem um ordenamento bem definido. Invertendo agora os limites de integração no segundo e quarto termos e os agrupando com o primeiro e terceiro, respectivamente, vem

$$
\begin{aligned}
D^{<}\left(t_{1}, t_{1}^{\prime}\right) & =\int_{-\infty}^{t_{1}} d t\left[A^{>}\left(t_{1}, t\right)-A^{<}\left(t_{1}, t\right)\right] B^{<}\left(t, t_{1}^{\prime}\right) \\
& +\int_{-\infty}^{t_{1}^{\prime}} d t A^{<}\left(t_{1}, t\right)\left[B^{<}\left(t, t_{1}^{\prime}\right)-B^{>}\left(t, t_{1}^{\prime}\right)\right] .
\end{aligned}
$$


Podemos estender os limites de integração até $+\infty$, utilizando funções degrau (step function) como abaixo,

$$
\begin{aligned}
D^{<}\left(t_{1}, t_{1}^{\prime}\right) & =\int_{-\infty}^{+\infty} d t \theta\left(t_{1}-t\right)\left[A^{>}\left(t_{1}, t\right)-A^{<}\left(t_{1}, t\right)\right] B^{<}\left(t, t_{1}^{\prime}\right) \\
& +\int_{-\infty}^{+\infty} d t A^{<}\left(t_{1}, t\right) \theta\left(t_{1}^{\prime}-t\right)\left[B^{<}\left(t, t_{1}^{\prime}\right)-B^{>}\left(t, t_{1}^{\prime}\right)\right] .
\end{aligned}
$$

Usando agora as definições de funções de Green avançada e retardada, Eqs.(2.20)(2.21) obtemos

$$
D^{<}\left(t_{1}, t_{1}^{\prime}\right)=\int_{-\infty}^{+\infty} d t A^{r}\left(t_{1}, t\right) B^{<}\left(t, t_{1}^{\prime}\right)+\int_{-\infty}^{+\infty} d t A^{<}\left(t_{1}, t\right) B^{a}\left(t, t_{1}^{\prime}\right) .
$$

Para a função de Green maior $D^{>}\left(t_{1}, t_{1}^{\prime}\right)$ basta trocar $<$ por $>$ na equação (2.29). Esse resultado pode ser facilmente generalizado para um produto de três funções de Green. Se tivermos, por exemplo, um produto de funções de Green $D=A B E^{4}$, obtemos para $D^{<}$o seguinte

$$
D^{<}=A^{r} B^{r} E^{<}+A^{r} B^{<} E^{a}+A^{<} B^{a} E^{a} .
$$

A função de Green retardada é obtida substituindo (2.29) e a respectiva expressão para $D^{>}\left(t_{1}, t_{1}^{\prime}\right)$ na Eq.(2.21). Com isso vem

$$
\begin{aligned}
D^{r}\left(t_{1}, t_{1}^{\prime}\right) & =\theta\left(t_{1}-t_{1}^{\prime}\right)\left[D^{>}\left(t_{1}, t_{1}^{\prime}\right)-D^{<}\left(t_{1}, t_{1}^{\prime}\right)\right] \\
& =\theta\left(t_{1}-t_{1}^{\prime}\right) \int_{-\infty}^{\infty} d t\left[A^{r}\left(B^{>}-B^{<}\right)+\left(A^{>}-A^{<}\right) B^{a}\right] \\
& =\theta\left(t_{1}-t_{1}^{\prime}\right) \int_{-\infty}^{\infty} d t\left[A^{r}\left(B^{r}-B^{a}\right)+\left(A^{r}-A^{a}\right) B^{a}\right] \\
& =\theta\left(t_{1}-t_{1}^{\prime}\right) \int_{-\infty}^{\infty} d t\left[A^{r} B^{r}-A^{a} B^{a}\right]=\int_{-\infty}^{\infty} d t A^{r}\left(t_{1}, t\right) B^{r}\left(t, t_{1}^{\prime}\right) .
\end{aligned}
$$

Portanto se $D=A B$, a função $D^{r}$ é simplesmente o produto das funções de Green retardada $A^{r}$ e $B^{r}$. Para a função de Green avançada temos analogamente $D^{a}\left(t_{1}, t_{1}^{\prime}\right)=\int_{-\infty}^{\infty} d t A^{a}\left(t_{1}, t\right) B^{a}\left(t, t_{1}^{\prime}\right)$. Em algumas análises precisamos ainda calcular $D^{\gtrless}$ ou $E^{\gtrless}$ onde $D\left(\tau_{1}, \tau_{1}^{\prime}\right)=A\left(\tau_{1}, \tau_{1}^{\prime}\right) B\left(\tau_{1}, \tau_{1}^{\prime}\right)$ e $E\left(\tau_{1}, \tau_{1}^{\prime}\right)=A\left(\tau_{1}, \tau_{1}^{\prime}\right) B\left(\tau_{1}^{\prime}, \tau\right)$. Os resultados são simplesmente [72]

$$
\begin{aligned}
& D^{\gtrless}\left(t_{1}, t_{1}^{\prime}\right)=A^{\gtrless}\left(t_{1}, t_{1}^{\prime}\right) B^{\gtrless}\left(t_{1}, t_{1}^{\prime}\right) \\
& E^{\gtrless}\left(t_{1}, t_{1}^{\prime}\right)=A^{\gtrless}\left(t_{1}, t_{1}^{\prime}\right) B^{\lessgtr}\left(t_{1}^{\prime}, t_{1}\right) .
\end{aligned}
$$

Essas equações constituem a base da continuação analítica desenvolvida por David Langreth [72]. A continuação analítica pode ser aplicada, por exemplo, na equação de Dyson (2.22) para encontrarmos $G^{<}, G^{>}, G^{r}$ e $G^{a}$. Seguimos calculando $G^{<}$, o que irá gerar a equação de Keldysh.

\footnotetext{
${ }^{4}$ Nessa notação compacta o produto de duas funções de Green corresponde a uma integração na variável temporal.
} 


\subsection{Equação de Keldysh}

A equação de Keldysh é a equação para $G^{<}$obtida a partir da equação de Dyson (2.22) via continuação analítica. Vimos na seção anterior que se a função de Green é um produto de três funções de Green. Aplicando a equação (2.30) na equação (2.22) obtemos

$$
G^{<}=G^{0<}+G^{0 r} \Sigma^{r} G^{<}+G^{0 r} \Sigma^{<} G^{a}+G^{0<} \Sigma^{a} G^{a} .
$$

Na equação acima adotamos a seguinte notação compacta: cada produto de funções de Green corresponde a uma integral temporal (e possivelmente espacial). Não escrevemos explicitamente as integrais para simplificar a notação e facilitar a leitura das equações na demonstração que se segue. As auto-energias $\Sigma^{r}, \Sigma^{a}$ e $\Sigma^{<}$também podem ser vistas como funções de Green para efeitos de aplicação das fórmulas de continuação analítica.

Substituindo a própria equação (2.34) no seu segundo termo, isto é, iterando uma vez, encontramos

$$
\begin{aligned}
G^{<} & =G^{0<}+G^{0 r} \Sigma^{r} G^{0<}+G^{0 r} \Sigma^{r} G^{0 r} \Sigma^{r} G^{<}+G^{0 r} \Sigma^{r} G^{0 r} \Sigma^{<} G^{a}+G^{0 r} \Sigma^{r} G^{0<} \Sigma^{a} G^{a} \\
& +G^{0 r} \Sigma^{<} G^{a}+G^{0<} \Sigma^{a} G^{a} .
\end{aligned}
$$

Substituindo agora $G^{<}$no terceiro termo (iterando uma segunda vez) vem

$$
\begin{aligned}
G^{<} & =G^{0<}+G^{0 r} \Sigma^{r} G^{0<}+G^{0 r} \Sigma^{r} G^{0 r} \Sigma^{r} G^{0<} \\
& +G^{0 r} \Sigma^{r} G^{0 r} \Sigma^{r} G^{0 r} \Sigma^{r} G^{0<}+G^{0 r} \Sigma^{r} G^{0 r} \Sigma^{r} G^{0 r} \Sigma^{r} G^{0 r} \Sigma^{r} G^{<} \\
& +G^{0 r} \Sigma^{r} G^{0 r} \Sigma^{r} G^{0 r} \Sigma^{r} G^{0 r} \Sigma^{<} G^{a}+G^{0 r} \Sigma^{r} G^{0 r} \Sigma^{r} G^{0 r} \Sigma^{r} G^{0<} \Sigma^{a} G^{a} \\
& +G^{0 r} \Sigma^{r} G^{0 r} \Sigma^{r} G^{0 r} \Sigma^{<} G^{a}+G^{0 r} \Sigma^{r} G^{0 r} \Sigma^{r} G^{0<} \Sigma^{a} G^{a} \\
& +G^{0 r} \Sigma^{r} G^{0 r} \Sigma^{<} G^{a}+G^{0 r} \Sigma^{r} G^{0<} \Sigma^{a} G^{a} \\
& +G^{0 r} \Sigma^{<} G^{a}+G^{0<} \Sigma^{a} G^{a} .
\end{aligned}
$$

Agrupando os termos denotados pela mesma cor na Eq.(2.36), obtemos

$$
\begin{aligned}
G^{<} & =G^{0<}+\left[G^{0 r}+G^{0 r} \Sigma^{r} G^{0 r}+G^{0 r} \Sigma^{r} G^{0 r} \Sigma^{r} G^{0 r}\right] \Sigma^{r} G^{0<} \\
& +\left[G^{0 r}+G^{0 r} \Sigma^{r} G^{0 r}+G^{0 r} \Sigma^{r} G^{0 r} \Sigma^{r} G^{0 r}\right] \Sigma^{r} G^{0<} \Sigma^{a} G^{a} \\
& +\left[G^{0 r}+G^{0 r} \Sigma^{r} G^{0 r}+G^{0 r} \Sigma^{r} G^{0 r} \Sigma^{r} G^{0 r}+G^{0 r} \Sigma^{r} G^{0 r} \Sigma^{r} G^{0 r} \Sigma^{r} G^{0 r}\right] \Sigma^{<} G^{a} \\
& +G^{0 r} \Sigma^{r} G^{0 r} \Sigma^{r} G^{0 r} \Sigma^{r} G^{0 r} \Sigma^{r} G^{<}+G^{0<} \Sigma^{a} G^{a}
\end{aligned}
$$

Podemos concluir da equação acima que uma iteração até infinita ordem resulta em

$$
G^{<}=G^{0<}+G^{r} \Sigma^{r} G^{0<}+G^{r} \Sigma^{r} G^{0<} \Sigma^{a} G^{a}+G^{r} \Sigma^{<} G^{a}+G^{0<} \Sigma^{a} G^{a} .
$$

Agrupando nessa equação os termos em preto, vermelho e azul obtemos

$$
G^{<}=\left[1+G^{r} \Sigma^{r}\right] G^{0<}\left[1+\Sigma^{a} G^{a}\right]+G^{r} \Sigma^{<} G^{a},
$$


que é a equação integral de Keldysh [57]. Comparando essa equação com a forma integral da equação de Boltzmann [73], reconhece-se que o primeiro termo corresponde ao decaimento do estado inicial do sistema, enquanto o segundo é o termo de espalhamento. Essa analogia entre as equações de Keldysh e de Boltzmann foi desenvolvida por A. P. Jauho [74].

Para completar a descrição do transporte precisamos das funções de Green $G^{r}$ e $G^{a}$. Aplicando as equações de Langreth na Eq.(2.22) obtemos

$$
G^{r, a}=G^{0 r, 0 a}+G^{0 r, 0 a} \Sigma^{r, a} G^{r, a} .
$$

As Eqs.(2.39) e (2.40) constituem a base da nossa descrição de transporte quântico em sistemas de ponto e poço quântico.

Nos próximos capítulos aplicaremos o formalismo descrito aqui para calcular a corrente e o ruído (ruído térmico e shot noise) em um sistema de ponto quântico acoplado a eletrodos ferromagnéticos (Caps.3-4). Também calcularemos corrente num sistema de ponto quântico acoplado a múltiplos terminais (Cap.5) e corrente e ruído em um poço quântico acoplado a contatos DMS (Cap.6). 


\section{Capítulo 3}

\section{Formulação do transporte para o sistema FM-PQ-FM}

\subsection{Introdução}

Aqui aplicaremos o formalismo de funções de Green de não equilíbrio (introduzido no capítulo anterior) para calcular corrente $I$ e ruído $S$ em um sistema de ponto quântico acoplado a terminais ferromagnéticos [Fig.(3.1)]. A interação de Coulomb e espalhamento de spin no ponto quântico são levados em conta. Derivamos equações para a corrente e para o ruído que permitem avaliar os efeitos da interação de Coulomb, do espalhamento de spin, da polarização e do alinhamento magnético (paralelo ou antiparalelo) dos eletrodos sobre $I$ e sobre $S$ [58].

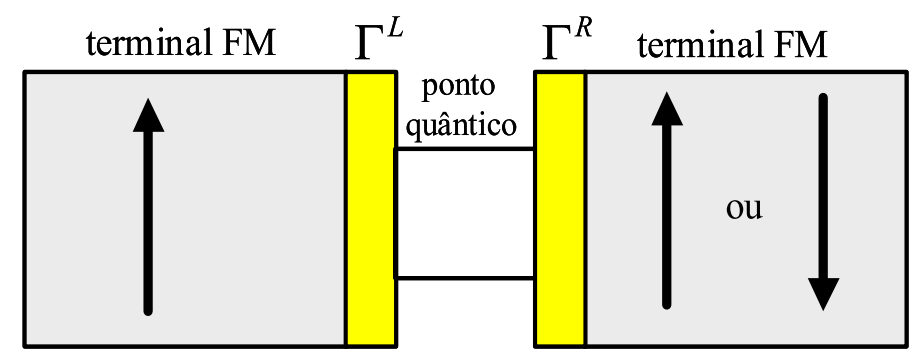

Figura 3.1: Ilustração de um sistema formado por um ponto quântico acoplado a dois terminais ferromagnéticos (FM-PQ-FM). Na presença de uma tensão $V$ surge uma corrente spin-polarizada e flutuações correspondentes. Nesse capítulo apresentamos o modelo utilizado para se estudar transporte (corrente e ruído) nesse sistema. 


\subsection{Modelo}

Para descrever o sistema da Fig.(3.1) utilizamos o seguinte Hamiltoniano

$$
H=H_{L}+H_{R}+H_{D}+H_{T}
$$

onde $H_{L}$ e $H_{R}$ descrevem os terminais da esquerda e da direita, respectivamente, $H_{D}$ o ponto quântico e $H_{T}$ é o termo de tunelamento. Note que os três primeiros termos $\left(H_{L}, H_{R}, H_{D}\right)$ correspondem a três regiões espaciais distintas, e $H_{T}$ hibridiza essas três regiões, permitindo assim a transição de elétrons de uma região a outra. Assim, o termo $H_{T}$ é responsável pela corrente elétrica na presença de uma tensão externa.

Mais especificamente, temos o seguinte Hamiltoniano para os terminais ferromagnéticos

$$
H_{\eta}=\sum_{\mathbf{k} \sigma} \epsilon_{\mathbf{k} \sigma \eta} c_{\mathbf{k} \sigma \eta}^{\dagger} c_{\mathbf{k} \sigma \eta},
$$

onde $\epsilon_{\mathbf{k} \sigma \eta}$ é a energia de um elétron na banda de condução do terminal de índice $\eta$, vetor de onda $\mathbf{k}$ e spin $\sigma$. Temos $\eta=L$ para o terminal da esquerda e $\eta=R$ para o terminal da direita. Assumimos que essa energia tem a forma $\epsilon_{\mathbf{k} \sigma \eta}=\epsilon_{\mathbf{k} \eta}+\sigma \Delta$, onde $\Delta$ dá o desdobramento das bandas para spin $\uparrow$ e $\downarrow$. O operador $c_{\mathbf{k} \sigma \eta}^{\dagger}\left(c_{\mathbf{k} \sigma \eta}\right)$ cria (destrói) um elétron com vetor de onda $\mathbf{k}$ e spin $\sigma$ no eletrodo $\eta$.

O Hamiltoniano para o ponto quântico é

$$
H_{D}=\sum_{\sigma} \epsilon_{\sigma} d_{\sigma}^{\dagger} d_{\sigma}+U n_{\uparrow} n_{\downarrow}+R\left(d_{\uparrow}^{\dagger} d_{\downarrow}+d_{\downarrow}^{\dagger} d_{\uparrow}\right)
$$

onde $\epsilon_{\sigma}$ é o nível do ponto quântico, e $d_{\sigma}\left(d_{\sigma}^{\dagger}\right)$ destrói (cria) um elétron com spin $\sigma$ no ponto quântico [75]. Consideramos um nível degenerado em spin $\epsilon_{\uparrow}=\epsilon_{\downarrow}=\epsilon_{d}$. A posição do nível $\epsilon_{d}$, com relação aos potenciais químicos do emissor $\mu_{L}$ e do coletor $\mu_{R}$, varia com a tensão $V$ entre eletrodos. Assumimos uma variação linear de tensão através da amostra, de modo que $\epsilon_{d}=\epsilon_{0}-e V / 2$, onde $e$ é a carga do elétron em módulo $(e>0)$, e $\epsilon_{0}$ o nível do ponto quântico para $V=0$. Os potenciais químicos $\mu_{L}$ e $\mu_{R}$ estão relacionados por $\mu_{L}-\mu_{R}=e V$. Em nossos cálculos $\mu_{L}$ é constante e define a origem de energia do sistema. A interação de Coulomb é levada em conta através do termo de Hubbard com parâmetro de correlação $U$. Esse termo dará origem ao bloqueio de Coulomb em nosso sistema. O último termo simula algum mecanismo de "espalhamento de spin", por exemplo, campos magnéticos girantes na geometria de electron spin resonance (ESR) [76].

Para efeitos de cálculo, é conveniente reescrever o Hamiltoniano (3.3) através da seguinte transformação [77]

$$
\begin{aligned}
& d_{\uparrow}=\frac{d_{1}+d_{2}}{\sqrt{2}} \\
& d_{\downarrow}=\frac{d_{2}-d_{1}}{\sqrt{2}},
\end{aligned}
$$


em que $d_{1}$ e $d_{2}$ são os operadores destruição na nova base $\{1,2\}$. De maneira compacta essa transformação pode ser expressa na seguinte forma

$$
d_{\sigma}=\frac{1}{\sqrt{2}} \sum_{i=1,2}(-1)^{i \delta_{\sigma \downarrow}} d_{i},
$$

onde $\delta_{\sigma \downarrow}$ é a delta de Kronecker.

Através dessa transformação o Hamiltoniano do ponto quântico assume a seguinte forma

$$
H_{D}=\sum_{i} \epsilon_{i} d_{i}^{\dagger} d_{i}+U n_{1} n_{2}
$$

onde $\epsilon_{i}=\epsilon_{d}+(-1)^{i} R$, e $n_{i}=d_{i}^{\dagger} d_{i}$. Observe que o espalhamento de spin introduz um desdobramento do nível $\epsilon_{d}$, isto é, $\epsilon_{d} \rightarrow \epsilon_{d} \pm R$.

Por último temos o termo de tunelamento, que é dado por

$$
H_{T}=\sum_{\mathbf{k} \sigma \eta}\left(t_{\mathbf{k} \sigma} c_{\mathbf{k} \sigma \eta}^{\dagger} d_{\sigma}+t_{\mathbf{k} \sigma}^{*} d_{\sigma}^{\dagger} c_{\mathbf{k} \sigma \eta}\right)
$$

onde o elemento de matriz $t_{\mathbf{k} \sigma}$ conecta um nível do contato a um nível do ponto quântico. Note que o spin se conserva no processo de tunelamento, isto é, o elemento de matriz $t_{\mathbf{k} \sigma}$ não mistura diferentes componentes de spin. Aplicando a transformação (3.5) em (3.7) obtemos

$$
H_{T}=\sum_{\mathbf{k} \sigma \eta i} \frac{(-1)^{i \delta_{\sigma \downarrow}}}{\sqrt{2}}\left\{t_{\mathbf{k} \sigma}^{*} d_{i}^{\dagger} c_{\mathbf{k} \eta \sigma}+t_{\mathbf{k} \eta \sigma} c_{\mathbf{k} \eta \sigma}^{\dagger} d_{i}\right\} .
$$

Com isso o Hamiltoniano total do sistema fica

$$
\begin{aligned}
H & =\sum_{\mathbf{k} \sigma \eta} \epsilon_{\mathbf{k} \sigma \eta} c_{\mathbf{k} \sigma \eta}^{\dagger} c_{\mathbf{k} \sigma \eta} \\
& +\sum_{i} \epsilon_{i} d_{i}^{\dagger} d_{i}+U n_{1} n_{2} \\
& +\sum_{\mathbf{k} \sigma \eta i} \frac{(-1)^{i \delta_{\sigma \downarrow}}}{\sqrt{2}}\left\{t_{\mathbf{k} \sigma}^{*} d_{i}^{\dagger} c_{\mathbf{k} \eta \sigma}+t_{\mathbf{k} \eta \sigma} c_{\mathbf{k} \eta \sigma}^{\dagger} d_{i}\right\}
\end{aligned}
$$

Com esse Hamiltoniano podemos calcular as propriedades de transporte do sistema, como corrente $I$ e ruído $S$. Vamos então derivar equações para $I$ e $S$ em termos de funções de Green do ponto quântico e dos terminais ferromagnéticos.

\subsection{Corrente}

Para calcularmos a corrente no terminal $\eta$, utilizamos a definição $I_{\eta}=-e\left\langle\dot{N}_{\eta}\right\rangle$, onde $N_{\eta}$ é o operador número total de elétrons no contato $\eta$, isto é, $N_{\eta}=\sum_{\mathbf{k} \sigma} c_{\mathbf{k} \sigma \eta}^{\dagger} c_{\mathbf{k} \sigma \eta}$. Utilizando a equação de Heisenberg $\dot{N}_{\eta}=(i / \hbar)\left[H, N_{\eta}\right]$, temos

$$
\dot{N}_{\eta}=(i / \hbar)\left[H_{\eta}, N_{\eta}\right]+(i / \hbar)\left[H_{D}, N_{\eta}\right]+(i / \hbar)\left[H_{T}, N_{\eta}\right]
$$


Sendo $\left[H_{\eta}, N_{\eta}\right]=0$ e $\left[H_{D}, N_{\eta}\right]=0$, nos resta $\dot{N}_{\eta}=(i / \hbar)\left[H_{T}, N_{\eta}\right]$, o que fornece

$$
\dot{N}_{\eta}=\frac{i}{\hbar} \sum_{\mathbf{k} \sigma i} \frac{(-1)^{i \delta_{\sigma \downarrow}}}{\sqrt{2}} t_{\mathbf{k} \sigma}^{*} d_{i}^{\dagger}(t) c_{\mathbf{k} \sigma \eta}(t)-\frac{i}{\hbar} \sum_{\mathbf{k} \sigma i} \frac{(-1)^{i \delta_{\sigma \downarrow}}}{\sqrt{2}} t_{\mathbf{k} \sigma} c_{\mathbf{k} \sigma \eta}^{\dagger}(t) d_{i}(t) .
$$

Substituindo esse resultado na definição da corrente vem

$$
\begin{aligned}
I_{\eta} & =\frac{e i}{\hbar}\left\{\sum_{\mathbf{k} \sigma i} t_{\mathbf{k} \sigma} \frac{(-1)^{i \delta_{\sigma \downarrow}}}{\sqrt{2}}\left\langle c_{\mathbf{k} \sigma \eta}^{\dagger}(t) d_{i}(t)\right\rangle-\sum_{\mathbf{k} \sigma} t_{\mathbf{k} \sigma}^{*} \frac{(-1)^{i \delta_{\sigma \downarrow}}}{\sqrt{2}}\left\langle d_{i}^{\dagger}(t) c_{\mathbf{k} \sigma \eta}(t)\right\rangle\right\} \\
& =\frac{2 e}{\hbar} \operatorname{Re} \sum_{\mathbf{k} \sigma i} t_{\mathbf{k} \sigma} \frac{(-1)^{i \delta_{\sigma \downarrow} \downarrow}}{\sqrt{2}} i\left\langle c_{\mathbf{k} \sigma \eta}^{\dagger}(t) d_{i}(t)\right\rangle .
\end{aligned}
$$

Definindo a função de Green menor (lesser) $G_{i \mathbf{k} \sigma \eta}^{<}(t, t)=i\left\langle c_{\mathbf{k} \sigma \eta}^{\dagger}(t) d_{i}(t)\right\rangle$, podemos reescrever (3.12) da seguinte forma

$$
I_{\eta}=\frac{2 e}{\hbar} \operatorname{Re} \sum_{\mathbf{k} \sigma i} t_{\mathbf{k} \sigma} \frac{(-1)^{i \delta_{\sigma \downarrow}}}{\sqrt{2}} G_{i \mathbf{k} \sigma \eta}^{<}(t, t) .
$$

O passo seguinte consiste em obter $G_{i \mathbf{k} \sigma \eta}^{<}(t, t)$ via continuação analítica da função de Green ordenada no contorno $G_{i \mathbf{k} \sigma \eta}\left(\tau, \tau^{\prime}\right)=-i\left\langle T_{c} d_{i}(\tau) c_{\mathbf{k} \sigma \eta}^{\dagger}\left(\tau^{\prime}\right)\right\rangle$. Os cálculos estão apresentados no apêndice A. O resultado obtido está dado abaixo,

$$
G_{i \mathbf{k} \sigma \eta}^{<}\left(t, t^{\prime}\right)=\sum_{i_{1}} t_{\mathbf{k} \sigma}^{*} \frac{(-1)^{i_{1} \delta_{\sigma \downarrow}}}{\sqrt{2}} \int d t_{1}\left[G_{i i_{1}}^{r}\left(t, t_{1}\right) g_{\mathbf{k} \sigma \eta}^{<}\left(t_{1}, t^{\prime}\right)+G_{i i_{1}}^{<}\left(t, t_{1}\right) g_{\mathbf{k} \sigma \eta}^{a}\left(t_{1}, t^{\prime}\right)\right],
$$

onde $G_{i i_{1}}^{r}\left(t, t_{1}\right)$ e $G_{i i_{1}}^{<}\left(t, t_{1}\right)$ são funções de Green do ponto quântico e $g_{\mathbf{k} \sigma \eta}^{<, a}\left(t_{1}, t^{\prime}\right)$ dos terminais. Essas funções de Green são definidas da seguinte forma

$$
\begin{aligned}
G_{i i_{1}}^{r}\left(t, t_{1}\right) & =-i \theta\left(t-t^{\prime}\right)\left\langle\left\{d_{i}(t), d_{i_{1}}^{\dagger}\left(t_{1}\right)\right\}\right\rangle \\
G_{i i_{1}}^{<}\left(t, t_{1}\right) & =i\left\langle d_{i_{1}}^{\dagger}\left(t_{1}\right) d_{i}(t)\right\rangle \\
g_{\mathbf{k} \sigma \eta}^{<}\left(t_{1}, t^{\prime}\right) & =i\left\langle\widetilde{c}_{\mathbf{k} \sigma \eta}^{\dagger}\left(t^{\prime}\right) \widetilde{c}_{\mathbf{k} \sigma \eta}\left(t_{1}\right)\right\rangle \\
g_{\mathbf{k} \sigma \eta}^{a}\left(t_{1}, t^{\prime}\right) & =i \theta\left(t^{\prime}-t_{1}\right)\left\langle\left\{\widetilde{c}_{\mathbf{k} \sigma \eta}\left(t_{1}\right), \widetilde{c}_{\mathbf{k} \sigma \eta}^{\dagger}\left(t^{\prime}\right)\right\}\right\rangle,
\end{aligned}
$$

onde o símbolo $\sim$ sobre os operadores dos terminais $\left(c_{\mathbf{k} \sigma \eta}\right)$ indica que esses estão na representação de interação, isto é, sua evolução temporal não depende do operador $H_{T}$. Portanto as funções de Green $g_{\mathbf{k} \sigma \eta}^{<}$e $g_{\mathbf{k} \sigma \eta}^{a}$ correspondem a elétrons livres, e são de fácil solução. A parte "difícil" do problema reside nas funções de Green do ponto quântico $G_{i i_{1}}^{r}$ e $G_{i i_{1}}^{<}$, visto que essas incluem acoplamento entre ponto quântico e contatos, espalhamento de spin e interação de Coulomb.

Substituindo a Eq.(3.14) na Eq.(3.13) encontramos

$$
\begin{aligned}
I_{\eta}=\frac{2 e}{\hbar} \operatorname{Re} \sum_{\mathbf{k} \sigma i i_{1}}\left|t_{\mathbf{k} \sigma}\right|^{2} \frac{(-1)^{i \delta_{\sigma \downarrow}}}{\sqrt{2}} \frac{(-1)^{i_{1} \delta_{\sigma \downarrow}}}{\sqrt{2}} \int d t_{1} \\
{\left[G_{i i_{1}}^{r}\left(t, t_{1}\right) g_{\mathbf{k} \sigma \eta}^{<}\left(t_{1}, t\right)+G_{i i_{1}}^{<}\left(t, t_{1}\right) g_{\mathbf{k} \sigma \eta}^{a}\left(t_{1}, t\right)\right] . }
\end{aligned}
$$


Definindo as auto-energias associadas ao acoplamento ponto-quântico-terminais

$$
\Sigma_{j i}^{\eta(a,<)}\left(t_{1}, t\right)=\frac{1}{2} \sum_{\mathbf{k} \sigma}\left|t_{\mathbf{k} \sigma}\right|^{2}(-1)^{(i+j) \delta_{\sigma \downarrow}} g_{\mathbf{k} \sigma \eta}^{(a,<)}\left(t_{1}, t\right),
$$

a Eq.(3.16) pode ser reescrita da seguinte forma

$$
I_{\eta}=\frac{2 e}{\hbar} \operatorname{Re} \sum_{i j} \int d t_{1}\left\{G_{i j}^{r}\left(t, t_{1}\right) \Sigma_{j i}^{\eta<}\left(t_{1}, t\right)+G_{i j}^{<}\left(t, t_{1}\right) \Sigma_{j i}^{\eta a}\left(t_{1}, t\right)\right\} .
$$

Para um Hamiltoniano independente do tempo, as funções de Green variam segundo a diferença $t-t_{1}$. Nesse caso a transformada de Fourier nos leva à seguinte equação

$$
\begin{aligned}
I_{\eta} & =\frac{2 e}{\hbar} \operatorname{Re} \int \frac{d \epsilon}{2 \pi} \sum_{i j}\left\{G_{i j}^{r}(\epsilon) \Sigma_{j i}^{\eta<}(\epsilon)+G_{i j}^{<}(\epsilon) \Sigma_{j i}^{\eta a}(\epsilon)\right\} \\
& =\frac{e}{\hbar} \int \frac{d \epsilon}{2 \pi} \sum_{i j}\left\{\left[G_{i j}^{r}(\epsilon)-G_{i j}^{a}(\epsilon)\right] \Sigma_{j i}^{\eta<}(\epsilon)+G_{i j}^{<}(\epsilon)\left[\Sigma_{j i}^{\eta a}(\epsilon)-\Sigma_{j i}^{\eta r}(\epsilon)\right]\right\} .
\end{aligned}
$$

Agora sendo

$$
\begin{aligned}
\sum_{j i}^{\eta<}(\epsilon) & =\frac{1}{2} \sum_{\mathbf{k} \sigma}\left|t_{\mathbf{k} \sigma}\right|^{2}(-1)^{(i+j) \delta_{\sigma \downarrow}} g_{\mathbf{k} \sigma \eta}^{<}(\epsilon) \\
& =\frac{1}{2} \sum_{\mathbf{k} \sigma}\left|t_{\mathbf{k} \sigma}\right|^{2}(-1)^{(i+j) \delta_{\sigma \downarrow}} 2 \pi i \delta\left(\epsilon-\epsilon_{\mathbf{k} \sigma \eta}\right) n_{\eta}(\epsilon),
\end{aligned}
$$

podemos definir $\Gamma_{\sigma}^{\eta}=2 \pi \sum_{\mathbf{k}}\left|t_{\mathbf{k} \sigma}\right|^{2} \delta\left(\epsilon-\epsilon_{\mathbf{k} \sigma \eta}\right)$, de modo a escrever

$$
\Sigma_{j i}^{\eta<}(\epsilon)=\frac{i}{2} n_{\eta}(\epsilon) \sum_{\sigma}(-1)^{(i+j) \delta_{\sigma \downarrow}} \Gamma_{\sigma}^{\eta}=i n_{\eta}(\epsilon) \Gamma_{j i}^{\eta}(\epsilon),
$$

onde $\Gamma_{j i}^{\eta}=(1 / 2) \sum_{\sigma}(-1)^{(i+j) \delta_{\sigma \downarrow}} \Gamma_{\sigma}^{\eta}$. A grandeza $\Gamma_{\sigma}^{\eta}$ fornece a taxa com que os elétrons de spin $\sigma$ tunelam entre o ponto quântico e o terminal $\eta$. Além disso temos que

$$
\begin{aligned}
\sum_{j i}^{\eta a}(\epsilon)-\Sigma_{j i}^{\eta r}(\epsilon) & =\frac{1}{2} \sum_{\mathbf{k} \sigma}\left|t_{\mathbf{k} \sigma}\right|^{2}(-1)^{(i+j) \delta_{\sigma \downarrow}}\left[g_{\mathbf{k} \sigma \eta}^{a}(\epsilon)-g_{\mathbf{k} \sigma \eta}^{r}(\epsilon)\right] \\
& =\frac{1}{2} \sum_{\mathbf{k} \sigma}\left|t_{\mathbf{k} \sigma}\right|^{2}(-1)^{(i+j) \delta_{\sigma \downarrow}}\left[\frac{1}{\epsilon-\epsilon_{\mathbf{k} \sigma \eta}-i \delta}-\frac{1}{\epsilon-\epsilon_{\mathbf{k} \sigma \eta}+i \delta}\right] \\
& =\frac{1}{2} \sum_{\mathbf{k} \sigma}\left|t_{\mathbf{k} \sigma}\right|^{2}(-1)^{(i+j) \delta_{\sigma \downarrow}} 2 \pi i \delta\left(\epsilon-\epsilon_{\mathbf{k} \sigma \eta}\right) \\
& =\frac{i}{2} \sum_{\sigma}(-1)^{(i+j) \delta_{\sigma \downarrow}} \Gamma_{\sigma}^{\eta} \\
& =i \Gamma_{j i}^{\eta} .
\end{aligned}
$$

Substituindo os resultados (3.21) e (3.22) em (3.19) encontramos

$$
I_{\eta}=\frac{i e}{\hbar} \int \frac{d \epsilon}{2 \pi} \sum_{i j}\left\{\left[G_{i j}^{r}(\epsilon)-G_{i j}^{a}(\epsilon)\right] n_{\eta}(\epsilon)+G_{i j}^{<}(\epsilon)\right\} \Gamma_{j i}^{\eta} .
$$


Reescrevendo (3.23) em termos matriciais obtemos a seguinte equação

$$
I_{\eta}=\frac{i e}{\hbar} \int \frac{d \epsilon}{2 \pi} \operatorname{Tr}\left\{\boldsymbol{\Gamma}^{\eta}\left\{\left[\mathbf{G}^{r}(\epsilon)-\mathbf{G}^{a}(\epsilon)\right] n_{\eta}(\epsilon)+\mathbf{G}^{<}(\epsilon)\right\}\right\},
$$

onde Tr denota o traço matricial, $\left[\mathbf{G}^{a, r,<}\right]_{i j}=G_{i j}^{a, r,<}$, e

$$
\Gamma^{\eta}=\frac{1}{2}\left(\begin{array}{cc}
\Gamma_{\uparrow}^{\eta}+\Gamma_{\downarrow}^{\eta} & \Gamma_{\uparrow}^{\eta}-\Gamma_{\downarrow}^{\eta} \\
\Gamma_{\uparrow}^{\eta}-\Gamma_{\downarrow}^{\eta} & \Gamma_{\uparrow}^{\eta}+\Gamma_{\downarrow}^{\eta}
\end{array}\right)
$$

Podemos ainda escrever para cada componente de spin o seguinte

$$
I_{\eta}^{\sigma}=\frac{i e}{\hbar} \int \frac{d \epsilon}{2 \pi} \operatorname{Tr}\left\{\frac{\Gamma_{\sigma}^{\eta}}{2}\left(\begin{array}{cc}
1 & (-1)^{\delta_{\sigma \downarrow}} \\
(-1)^{\delta_{\sigma \downarrow}} & 1
\end{array}\right)\left[n_{\eta}\left(\mathbf{G}^{r}-\mathbf{G}^{a}\right)+\mathbf{G}^{<}\right]\right\},
$$

de modo que $I_{\eta}=I_{\eta}^{\uparrow}+I_{\eta}^{\downarrow}$.

\subsection{Expressão para a corrente no caso $U=0$ e $R=0$}

Se trabalharmos diretamente na base $\{\uparrow, \downarrow\}$ ao invés da base $\{1,2\}$ dada pela transformação (3.5), a equação para a corrente pode ser expressa numa forma idêntica a Eq.(3.24),

$$
I_{\eta}=\frac{i e}{\hbar} \int \frac{d \epsilon}{2 \pi} \operatorname{Tr}\left\{\boldsymbol{\Gamma}_{\uparrow \downarrow}^{\eta}\left\{\left[\mathbf{G}^{r}(\epsilon)-\mathbf{G}^{a}(\epsilon)\right] n_{\eta}(\epsilon)+\mathbf{G}^{<}(\epsilon)\right\}\right\},
$$

exceto pela matriz $\Gamma_{\uparrow \downarrow}^{\eta}$ que é dada por

$$
\Gamma_{\uparrow \downarrow}^{\eta}=\left(\begin{array}{cc}
\Gamma_{\uparrow}^{\eta} & 0 \\
0 & \Gamma_{\downarrow}^{\eta}
\end{array}\right)
$$

e as funções de Green tem a seguinte forma

$$
\mathbf{G}^{r, a,<}=\left(\begin{array}{cc}
G_{\uparrow \uparrow}^{r, a,<} & 0 \\
0 & G_{\downarrow \downarrow}^{r, a,<}
\end{array}\right) .
$$

As funções de Green são diagonais visto que consideramos $R=0$ (espalhamento de spin nulo). Para o caso não interagente e sem espalhamento de spin a função de Green retardada é simplesmente $G_{\sigma \sigma}^{r}=1 /\left(\epsilon-\epsilon_{\sigma}+i \Gamma_{\sigma} / 2\right)$, onde $\Gamma_{\sigma}=\Gamma_{\sigma}^{L}+\Gamma_{\sigma}^{R}$. Com isso temos

$$
\begin{aligned}
G_{\sigma \sigma}^{r}-G_{\sigma \sigma}^{a} & =\frac{1}{\epsilon-\epsilon_{\sigma}+i \Gamma_{\sigma} / 2}-\frac{1}{\epsilon-\epsilon_{\sigma}-i \Gamma_{\sigma} / 2} \\
& =\frac{-i \Gamma_{\sigma}}{\left(\epsilon-\epsilon_{\sigma}\right)^{2}+\left(\frac{\Gamma_{\sigma}}{2}\right)^{2}} .
\end{aligned}
$$


Também temos

$$
\begin{aligned}
G_{\sigma \sigma}^{<} & =G_{\sigma \sigma}^{r} \Sigma_{\sigma \sigma}^{<} G_{\sigma \sigma}^{a}=\frac{1}{\epsilon-\epsilon_{\sigma}+i \Gamma_{\sigma} / 2} i\left(n_{L} \Gamma_{\sigma}^{L}+n_{R} \Gamma_{\sigma}^{R}\right) \frac{1}{\epsilon-\epsilon_{\sigma}-i \Gamma_{\sigma} / 2} \\
& =i \frac{n_{L} \Gamma_{\sigma}^{L}+n_{R} \Gamma_{\sigma}^{R}}{\left(\epsilon-\epsilon_{\sigma}\right)^{2}+\left(\frac{\Gamma_{\sigma}}{2}\right)^{2}}
\end{aligned}
$$

Substituindo (3.30) e (3.31) na Eq.(3.27) obtemos para $I_{L}(\eta=L)$

$$
\begin{aligned}
I_{L} & =\frac{i e}{\hbar} \int \frac{d \epsilon}{2 \pi} \sum_{\sigma} \Gamma_{\sigma}^{L}\left[\frac{-i\left(\Gamma_{\sigma}^{L}+\Gamma_{\sigma}^{R}\right) n_{L}}{\left(\epsilon-\epsilon_{\sigma}\right)^{2}+\left(\frac{G_{\sigma}}{2}\right)}+i \Gamma_{\sigma}^{L} \frac{n_{L} \Gamma_{\sigma}^{L}+n_{R} \Gamma_{\sigma}^{R}}{\left(\epsilon-\epsilon_{\sigma}\right)^{2}+\left(\frac{\Gamma_{\sigma}}{2}\right)^{2}}\right] \\
& =\frac{e}{\hbar} \int \frac{d \epsilon}{2 \pi} \sum_{\sigma}\left(n_{L}-n_{R}\right) \frac{\Gamma_{\sigma}^{L} \Gamma_{\sigma}^{R}}{\left(\epsilon-\epsilon_{\sigma}\right)^{2}+\left(\frac{\Gamma_{\sigma}}{2}\right)^{2}} .
\end{aligned}
$$

Definindo $T_{0}^{\sigma}=\Gamma_{\sigma}^{L} \Gamma_{\sigma}^{R} /\left[\left(\epsilon-\epsilon_{\sigma}\right)^{2}+\left(\Gamma_{\sigma} / 2\right)^{2}\right]$, podemos escrever a corrente assim

$$
I_{L}=\frac{e}{\hbar} \int d \epsilon \sum_{\sigma} T_{0}^{\sigma}\left(n_{L}-n_{R}\right)
$$

que é formalmente idêntica a equação de Landauer-Büttiker [64]. Para tensões grande o suficiente aproximamos $n_{L}=1$ e $n_{R}=0$ na equação acima, que, ao ser integrada, resulta em

$$
I_{L}=\frac{e}{\hbar} \sum_{\sigma} \frac{\Gamma_{\sigma}^{L} \Gamma_{\sigma}^{R}}{\Gamma_{\sigma}^{L}+\Gamma_{\sigma}^{R}},
$$

que é uma expressão bastante conhecida na literatura para sistemas de dupla barreira em regime de tunelamento ressonante [64].

Utilizando a mesma metodologia de cálculo, seguimos apresentando uma forma sistemática de se obter o ruído na presença de terminais ferromagnéticos e espalhamento de spin no interior do ponto quântico.

\subsection{Ruído}

Nesta seção derivamos uma equação para o ruído que inclui espalhamento de spin e interação de Coulomb dentro do ponto quântico, além de levar em conta os terminais ferromagnéticos. Matematicamente o ruído é definido como a função auto-correlação dos operadores de flutuação de corrente

$$
S_{\eta \eta^{\prime}}=\left\langle\left\{\delta \widehat{I}_{\eta}(t), \delta \widehat{I}_{\eta^{\prime}}\left(t^{\prime}\right)\right\}\right\rangle=\left\langle\left\{\hat{I}_{\eta}(t), \hat{I}_{\eta^{\prime}}\left(t^{\prime}\right)\right\}\right\rangle-2 I_{\eta} I_{\eta^{\prime}}
$$

onde $\delta \widehat{I}_{\eta}(t)=\widehat{I}_{\eta}(t)-I_{\eta}$, sendo $I_{\eta}=\left\langle\widehat{I}_{\eta}(t)\right\rangle$. Substituindo o operador corrente

$$
\widehat{I}_{\eta}=\frac{e i}{\hbar}\left\{\sum_{\mathbf{k} \sigma i} t_{\mathbf{k} \sigma} \frac{(-1)^{i \delta_{\sigma \downarrow}}}{\sqrt{2}} c_{\mathbf{k} \sigma \eta}^{\dagger}(t) d_{i}(t)-\sum_{\mathbf{k} \sigma i} t_{\mathbf{k} \sigma}^{*} \frac{(-1)^{i \delta_{\sigma \downarrow}}}{\sqrt{2}} d_{i}^{\dagger}(t) c_{\mathbf{k} \sigma \eta}(t)\right\}
$$


em (3.35) obtemos

$$
\begin{aligned}
S_{\eta \eta^{\prime}}\left(t, t^{\prime}\right)= & \left(\frac{i e}{\hbar}\right)^{2} \sum_{\mathbf{k} \mathbf{k}^{\prime} \sigma \sigma^{\prime} i j} \frac{1}{2}(-1)^{i \delta_{\sigma \downarrow}}(-1)^{j \delta_{\sigma^{\prime} \downarrow}} \\
& \left\{t_{\mathbf{k} \sigma} t_{\mathbf{k}^{\prime} \sigma^{\prime}}\left\langle c_{\mathbf{k} \sigma \eta}^{\dagger}(t) d_{i}(t) c_{\mathbf{k}^{\prime} \sigma^{\prime} \eta^{\prime}}^{\dagger}\left(t^{\prime}\right) d_{j}\left(t^{\prime}\right)\right\rangle-t_{\mathbf{k} \sigma} t_{\mathbf{k}^{\prime} \sigma^{\prime}}^{*}\left\langle c_{\mathbf{k} \sigma \eta}^{\dagger}(t) d_{i}(t) d_{j}^{\dagger}\left(t^{\prime}\right) c_{\mathbf{k}^{\prime} \sigma^{\prime} \eta^{\prime}}\left(t^{\prime}\right)\right\rangle\right. \\
& \left.-t_{\mathbf{k} \sigma}^{*} t_{\mathbf{k}^{\prime} \sigma^{\prime}}\left\langle d_{i}^{\dagger}(t) c_{\mathbf{k} \sigma \eta}(t) c_{\mathbf{k}^{\prime} \sigma^{\prime} \eta^{\prime}}^{\dagger}\left(t^{\prime}\right) d_{j}\left(t^{\prime}\right)\right\rangle+t_{\mathbf{k} \sigma^{*}}^{*} t_{\mathbf{k}^{\prime} \sigma^{\prime}}^{*}\left\langle d_{i}^{\dagger}(t) c_{\mathbf{k} \sigma \eta}(t) d_{j}^{\dagger}\left(t^{\prime}\right) c_{\mathbf{k}^{\prime} \sigma^{\prime} \eta^{\prime}}\left(t^{\prime}\right)\right\rangle\right\} \\
& + \text { h.c. }-2 I_{\eta} I_{\eta^{\prime}} .
\end{aligned}
$$

Definindo as funções de Green abaixo

$$
\begin{aligned}
g_{1}^{(2)>}\left(t, t^{\prime}\right) & =i^{2}\left\langle c_{\mathbf{k} \sigma \eta}^{\dagger}(t) d_{i}(t) c_{\mathbf{k}^{\prime} \sigma^{\prime} \eta^{\prime}}^{\dagger}\left(t^{\prime}\right) d_{j}\left(t^{\prime}\right)\right\rangle \\
g_{2}^{(2)>}\left(t, t^{\prime}\right) & =i^{2}\left\langle c_{\mathbf{k} \sigma \eta}^{\dagger}(t) d_{i}(t) d_{j}^{\dagger}\left(t^{\prime}\right) c_{\mathbf{k}^{\prime} \sigma^{\prime} \eta^{\prime}}\left(t^{\prime}\right)\right\rangle \\
g_{3}^{(2)>}\left(t, t^{\prime}\right) & =i^{2}\left\langle d_{i}^{\dagger}(t) c_{\mathbf{k} \sigma \eta}(t) c_{\mathbf{k}^{\prime} \sigma^{\prime} \eta^{\prime}}^{\dagger}\left(t^{\prime}\right) d_{j}\left(t^{\prime}\right)\right\rangle \\
g_{4}^{(2)>}\left(t, t^{\prime}\right) & =i^{2}\left\langle d_{i}^{\dagger}(t) c_{\mathbf{k} \sigma \eta}(t) d_{j}^{\dagger}\left(t^{\prime}\right) c_{\mathbf{k}^{\prime} \sigma^{\prime} \eta^{\prime}}\left(t^{\prime}\right)\right\rangle
\end{aligned}
$$

podemos reescrever a Eq.(3.37) da seguinte forma

$$
\begin{aligned}
S_{\eta \eta^{\prime}}\left(t, t^{\prime}\right)= & \left(\frac{e}{\hbar}\right)^{2} \sum_{\mathbf{k}^{\prime} \sigma \sigma^{\prime} i j} \frac{1}{2}(-1)^{i \delta_{\sigma \downarrow}}(-1)^{j \delta_{\sigma^{\prime} \downarrow}} \\
& \left\{t_{\mathbf{k} \sigma} t_{\mathbf{k}^{\prime} \sigma^{\prime}} g_{1}^{(2)>}\left(t, t^{\prime}\right)-t_{\mathbf{k} \sigma} t_{\mathbf{k}^{\prime} \sigma^{\prime}}^{*} g_{2}^{(2)>}\left(t, t^{\prime}\right)-\right. \\
& \left.t_{\mathbf{k} \sigma}^{*} t_{\mathbf{k}^{\prime} \sigma^{\prime}} g_{3}^{(2)>}\left(t, t^{\prime}\right)+t_{\mathbf{k} \sigma}^{*} t_{\mathbf{k}^{\prime} \sigma^{\prime}}^{*} g_{4}^{(2)>}\left(t, t^{\prime}\right)\right\} \\
& + \text { h.c. }-2 I_{\eta} I_{\eta^{\prime}} .
\end{aligned}
$$

Para obtermos as funções de Green $g_{i}^{(2)>}\left(t, t^{\prime}\right)$ adotamos o mesmo procedimento algébrico utilizado no cálculo de $G_{i \mathbf{k} \sigma \eta}^{<}\left(t, t^{\prime}\right)$ [veja Eq.(3.14)]. Lembre-se que para $G_{i \mathbf{k} \sigma \eta}^{<}\left(t, t^{\prime}\right)$ seguimos os seguintes passos: (1) definimos uma função de Green ordenada no contorno $G_{i \mathbf{k} \sigma \eta}\left(\tau, \tau^{\prime}\right)$, (2) expandimos a matriz $S$ e reagrupamos termos, e (3) aplicamos as regras de Langreth para encontrar $G_{i \mathbf{k} \sigma \eta}^{<}\left(t, t^{\prime}\right)$. Agora, (1) definire$\operatorname{mos} g_{i}^{(2)}\left(\tau, \tau^{\prime}\right)$, (2) faremos a expansão da matriz $S$ e reagruparemos termos, e (3) obteremos $g_{i}^{(2)>}\left(t, t^{\prime}\right)$ via continuação analítica. Como o cálculo é longo optamos por apresentá-lo no apêndice B. Aqui simplesmente mostramos o resultado final para o ruído obtido a partir da Eq.(3.42). Para o caso $\eta=\eta^{\prime}=L$ temos a seguinte equação no limite de baixas freqüências [79]

$$
\begin{aligned}
S_{L L}(\omega \rightarrow 0) & =\frac{2 e^{2}}{\hbar} \int \frac{d \epsilon}{2 \pi} \operatorname{Tr}\left\{\left\{i n_{L} \boldsymbol{\Gamma}^{L} \mathbf{G}^{>}-i\left[1-n_{L}\right] \boldsymbol{\Gamma}^{L} \mathbf{G}^{<}\right\}\right. \\
& +\left(\mathbf{G}^{r}-\mathbf{G}^{a}\right)\left(n_{L} \boldsymbol{\Gamma}^{L} \mathbf{G}^{>} \boldsymbol{\Gamma}^{L}-\left[1-n_{L}\right] \boldsymbol{\Gamma}^{L} \mathbf{G}^{<} \boldsymbol{\Gamma}^{L}\right) \\
& \left.-n_{L}\left[1-n_{L}\right]\left(\mathbf{G}^{a} \boldsymbol{\Gamma}^{L} \mathbf{G}^{a} \boldsymbol{\Gamma}^{L}+\mathbf{G}^{r} \boldsymbol{\Gamma}^{L} \mathbf{G}^{r} \boldsymbol{\Gamma}^{L}\right)+\mathbf{G}^{<} \boldsymbol{\Gamma}^{L} \mathbf{G}^{>} \boldsymbol{\Gamma}^{L}\right\} .
\end{aligned}
$$

O limite $\omega \rightarrow 0$ significa que estamos numa escala de freqüência muito menor do que o recíproco do tempo de transito dos elétrons pelo sistema (tipicamente $\sim$ $\mathrm{GHz}$ ). Do ponto de vista experimental, o limite de freqüência nula corresponde a freqüências entre $1 \mathrm{kHz}$ e $100 \mathrm{kHz}$, visto que o ruído só irá apresentar alguma 
estrutura em freqüências muito maiores do que essas. É importante ressaltar que em geral para freqüências menores do que $1 \mathrm{kHz}$ o ruído $1 / f$ predomina. Portanto o limite $\omega \rightarrow 0$ não corresponde estritamente a freqüência nula, mas sim freqüências da ordem de $1 \mathrm{kHz}$ a $100 \mathrm{kHz}$, que é uma faixa de freqüências em que o ruído é aproximadamente constante. Medidas experimentais do ruído como função da freqüência podem ser vistas em [46] para um sistema de dupla-barreira de tunelamento ressonante, em [52] para um sistema de pontos quânticos autoorganizados, e em [53] para um sistema de um único ponto quântico.

Pode-se constatar nesses experimentos uma faixa de freqüência em que o ruído é aproximadamente constante, e a divergência em torno de $\omega=0$ proveniente do ruído $1 / f$.

Na equação (3.43) apresentamos apenas a componente $S_{L L}$ do ruído. As demais componentes $S_{R R}, S_{L R}$ e $S_{R L}$ estão apresentadas no apêndice B. Vale salientar que basta calcular uma dessas componentes, por exemplo $S_{L L}$, pois $S_{L L}=S_{R R}=-S_{L R}=-S_{R L}$. Algumas vezes ao longo do texto nos referimos ao ruído simplesmente por $S$, sendo $S=S_{L L}=S_{R R}=-S_{L R}=-S_{R L}$.

$\mathrm{Na}$ ausência de interação de Coulomb $(U=0)$ o Hamiltoniano (3.9) fica quadrático, e com isso a expressão (3.43) torna-se exata, levando em conta espalhamento de spin e terminais ferromagnéticos. Para $U \neq 0$, porém, a Eq.(3.43) é aproximada. Conforme discutimos no apêndice B, para obtermos (3.43) truncamos a expansão diagramática da função de Green $g_{i}^{(2)}\left(\tau, \tau^{\prime}\right)$, quebrando médias do tipo $\left\langle T_{c} d_{i}(\tau) d_{j}^{\dagger}\left(\tau^{\prime}\right) d_{i_{2}}\left(\tau_{2}\right) d_{i_{1}}^{\dagger}\left(\tau_{1}\right)\right\rangle$ em produto de médias de dois operadores, por exemplo, $\left\langle T_{c} d_{i}(\tau) d_{j}^{\dagger}\left(\tau^{\prime}\right)\right\rangle\left\langle T_{c} d_{i_{2}}\left(\tau_{2}\right) d_{i_{1}}^{\dagger}\left(\tau_{1}\right)\right\rangle$. Esse procedimento só é exato para $U=0$. Na seção seguinte, vamos deduzir uma versão simplificada da Eq.(3.43) para o caso $U=0$ e $R=0$.

\subsection{Expressão para o ruído no caso $U=0$ e $R=0$}

Na ausência de espalhamento de spin $(R=0)$ e interação de Coulomb $(U=0)$ temos a seguinte expressão exata para o ruído

$$
\begin{aligned}
S_{L L}(\omega \rightarrow 0) & =\frac{2 e^{2}}{\hbar} \int \frac{d \epsilon}{2 \pi} \operatorname{Tr}\left\{\left\{i n_{L} \boldsymbol{\Gamma}_{\uparrow \downarrow}^{L} \mathbf{G}^{>}-i\left[1-n_{L}\right] \boldsymbol{\Gamma}_{\uparrow \downarrow}^{L} \mathbf{G}^{<}\right\}\right. \\
& +\left(\mathbf{G}^{r}-\mathbf{G}^{a}\right)\left(n_{L} \boldsymbol{\Gamma}_{\uparrow \downarrow}^{L} \mathbf{G}^{>} \boldsymbol{\Gamma}_{\uparrow \downarrow}^{L}-\left[1-n_{L}\right] \boldsymbol{\Gamma}_{\uparrow \downarrow}^{L} \mathbf{G}^{<} \boldsymbol{\Gamma}_{\uparrow \downarrow}^{L}\right) \\
& \left.-n_{L}\left[1-n_{L}\right]\left(\mathbf{G}^{a} \boldsymbol{\Gamma}_{\uparrow \downarrow}^{L} \mathbf{G}^{a} \boldsymbol{\Gamma}_{\uparrow \downarrow}^{L}+\mathbf{G}^{r} \boldsymbol{\Gamma}_{\uparrow \downarrow}^{L} \mathbf{G}^{r} \boldsymbol{\Gamma}_{\uparrow \downarrow}^{L}\right)+\mathbf{G}^{<} \boldsymbol{\Gamma}_{\uparrow \downarrow}^{L} \mathbf{G}^{>} \boldsymbol{\Gamma}_{\uparrow \downarrow}^{L}\right\},
\end{aligned}
$$

onde as funções de Green são dadas pela Eq.(3.29) e a matriz $\Gamma_{\uparrow \downarrow}^{L}$ é dada pela Eq.(3.28). Note que aqui optamos por trabalhar na base $\{\uparrow, \downarrow\}$ ao invés da base $\{1,2\}$, visto que não há espalhamento de spin para acoplar os canais $\uparrow$ e $\downarrow$. No regime $k_{B} T<<\mathrm{eV}$ (altas tensões) podemos aproximar $1-n_{L}=0$ e $n_{L}=1 \mathrm{de}$ 
modo que a Eq.(3.44) fica

$$
\begin{aligned}
S_{L L}(0) & =\frac{2 e^{2}}{\hbar} \int \frac{d \epsilon}{2 \pi} \sum_{\sigma}\left\{i \Gamma_{\sigma}^{L} G_{\sigma \sigma}^{>}+\right. \\
& =\frac{2 e^{2}}{\hbar} \int \frac{d \epsilon}{2 \pi} \sum_{\sigma} i \Gamma_{\sigma}^{L} G_{\sigma \sigma}^{>}\left\{1-i\left(G_{\sigma \sigma}^{r}-G_{\sigma \sigma}^{a}\right) \Gamma_{\sigma}^{L}+i G_{\sigma \sigma}^{<} \Gamma_{\sigma}^{L}\right\} \\
& =\frac{2 e^{2}}{\hbar} \int \frac{d \epsilon}{2 \pi} \sum_{\sigma} i \Gamma_{\sigma}^{L} G_{\sigma \sigma}^{>}\left\{1-i \Gamma_{\sigma}^{L} G_{\sigma \sigma}^{>}\right\}
\end{aligned}
$$

onde usamos o fato de $G_{\sigma \sigma}^{>}=G_{\sigma \sigma}^{r}-G_{\sigma \sigma}^{a}+G_{\sigma \sigma}^{<}$. Definindo $T_{0}^{\sigma}=i \Gamma_{\sigma}^{L} G_{\sigma \sigma}^{>}$, podemos escrever

$$
S_{L L}(0)=\frac{2 e^{2}}{\hbar} \int \frac{d \epsilon}{2 \pi} \sum_{\sigma} T_{0}^{\sigma}\left(1-T_{0}^{\sigma}\right),
$$

que é a fórmula de Landauer-Büttiker para o ruído [64]. Substituindo a expressão do coeficiente de transmissão,

$$
T_{0}^{\sigma}=i \Gamma_{\sigma}^{L} G_{\sigma \sigma}^{>}=i \Gamma_{\sigma}^{L}\left\{G_{\sigma \sigma}^{r}-G_{\sigma \sigma}^{a}+G_{\sigma \sigma}^{<}\right\}=\frac{\Gamma_{\sigma}^{L} \Gamma_{\sigma}^{R}}{\left(\epsilon-\epsilon_{\sigma}\right)^{2}+\left(\frac{\Gamma_{\sigma}}{2}\right)^{2}},
$$

na equação do ruído (3.46), e efetuando a integração obtemos

$$
S_{L L}=\frac{2 e^{2}}{\hbar} \sum_{\sigma} \frac{\Gamma_{\sigma}^{L} \Gamma_{\sigma}^{R}\left(\Gamma_{\sigma}^{L^{2}}+\Gamma_{\sigma}^{R^{2}}\right)}{\left(\Gamma_{\sigma}^{L}+\Gamma_{\sigma}^{R}\right)^{3}} .
$$

Esse resultado é bastante familiar dentro da literatura sobre ruído [64]. Com isso vemos que a nossa equação (3.43) contém, em um determinado regime $(U=0$, $\left.R=0, k_{B} T<<e V\right)$, resultados usuais da literatura.

\subsection{Funções de Green do Ponto Quântico}

Para se utilizar as Eqs.(3.24) e (3.43) precisamos conhecer as diferentes funções de Green do ponto quântico, isto é, $\mathbf{G}^{r}, \mathbf{G}^{a}, \mathbf{G}^{<}$e $\mathbf{G}^{>}$. Essas funções de Green são obtidas a partir de $\mathbf{G}\left(\tau, \tau^{\prime}\right)$ via continuação analítica. Como $\mathbf{G}\left(\tau, \tau^{\prime}\right)$ é estruturalmente idêntica a $\mathbf{G}\left(t, t^{\prime}\right)$, calculamos uma equação para $\mathbf{G}\left(t, t^{\prime}\right)$ e em seguida passamos das variáveis $\left(t, t^{\prime}\right)$ para os tempos ordenados ao longo do contorno $\left(\tau, \tau^{\prime}\right)$.

Comecemos então calculando $\mathbf{G}\left(t, t^{\prime}\right)$. Derivando $G_{i j}\left(t, t^{\prime}\right)=-i\left\langle T d_{i}(t) d_{j}^{\dagger}\left(t^{\prime}\right)\right\rangle$ com relação ao tempo $t$, obtemos a seguinte equação de movimento

$$
\left(i \frac{\partial}{\partial t}-\epsilon_{i}\right) G_{i j}\left(t, t^{\prime}\right)=\delta_{i j} \delta\left(t-t^{\prime}\right)+U G_{i j}^{(2)}\left(t, t^{\prime}\right)+\sum_{i_{1}} \int d t_{1} \Sigma_{i i_{1}}\left(t, t_{1}\right) G_{i_{1} j}\left(t_{1}, t^{\prime}\right)
$$

onde $G_{i j}^{(2)}\left(t, t^{\prime}\right)=-i\left\langle T n_{\bar{i}}(t) d_{i}(t) d_{j}^{\dagger}\left(t^{\prime}\right)\right\rangle$, e

$$
\Sigma_{i i_{1}}\left(t, t_{1}\right)=\sum_{\mathbf{k} \sigma \eta} \frac{(-1)^{\left(i+i_{1}\right) \delta_{\sigma \downarrow}}}{2}\left|t_{\mathbf{k} \sigma}\right|^{2} g_{\mathbf{k} \sigma \eta}\left(t, t_{1}\right),
$$


sendo $g_{\mathbf{k} \sigma \eta}\left(t, t_{1}\right)=-i\left\langle T \widetilde{c}_{\mathbf{k} \sigma \eta}(t) \widetilde{c}_{\mathbf{k} \sigma \eta}^{\dagger}\left(t_{1}\right)\right\rangle$ a função de Green dos elétrons livres nos contatos ferromagnéticos. Calculando agora a equação de movimento para $G_{i j}^{(2)}\left(t, t^{\prime}\right)$ encontramos

$$
\begin{aligned}
\left(i \frac{\partial}{\partial t}-\epsilon_{i}-U\right) G_{i j}^{(2)}\left(t, t^{\prime}\right) & =\delta\left(t-t^{\prime}\right)\left[\delta_{i j}\left\langle n_{\bar{i}}\right\rangle-\delta_{\bar{i} j}\left\langle d_{\bar{i}}^{\dagger} d_{i}\right\rangle\right] \\
& +\sum_{\mathbf{k} \sigma \eta} \frac{(-1)^{\bar{i} \delta_{\sigma \downarrow}}}{\sqrt{2}} t_{\mathbf{k} \sigma}^{*}(-i)\left\langle T d_{\bar{i}}^{\dagger}(t) c_{\mathbf{k} \sigma \eta}(t) d_{i}(t) d_{j}^{\dagger}\left(t^{\prime}\right)\right\rangle \\
& +\sum_{\mathbf{k} \sigma \eta} \frac{(-1)^{i \delta \sigma \downarrow}}{\sqrt{2}} t_{\mathbf{k} \sigma}^{*}(-i)\left\langle T n_{\bar{i}}(t) c_{\mathbf{k} \sigma \eta}(t) d_{j}^{\dagger}\left(t^{\prime}\right)\right\rangle \\
& -\sum_{\mathbf{k} \sigma \eta} \frac{(-1)^{\bar{i} \delta_{\sigma \downarrow}}}{\sqrt{2}} t_{\mathbf{k} \sigma}(-i)\left\langle T c_{\mathbf{k} \sigma \eta}^{\dagger}(t) d_{\bar{i}}(t) d_{i}(t) d_{j}^{\dagger}\left(t^{\prime}\right)\right\rangle
\end{aligned}
$$

O próximo passo seria calcular as equações de movimento para cada uma das três funções de Green em (3.51). Esse procedimento geraria novas funções de Green que por sua vez precisariam ser calculadas pelo método de equações de movimento, e assim sucessivamente. Desse modo teríamos um sistema infinito de equações diferenciais acopladas. Em algum momento então precisamos truncar essa expansão de modo a fechar o sistema de equações. Optamos por truncá-la no presente estágio do cálculo, em virtude de uma série de complicações matemáticas que surgem em aproximações de mais alta ordem. Desse modo fazemos a seguinte aproximação [57]

$$
\begin{aligned}
\left\langle T d_{\bar{i}}(t) d_{i}(t) c_{\mathbf{k} \sigma \eta}^{\dagger}(t) d_{j}^{\dagger}\left(t^{\prime}\right)\right\rangle & =0 \\
\left\langle T d_{\bar{i}}^{\dagger}(t) d_{i}(t) c_{\mathbf{k} \sigma \eta}(t) d_{j}^{\dagger}\left(t^{\prime}\right)\right\rangle & =\left\langle d_{\bar{i}}^{\dagger} d_{i}\right\rangle\left\langle T c_{\mathbf{k} \sigma \eta}(t) d_{j}^{\dagger}\left(t^{\prime}\right)\right\rangle \\
\left\langle T d_{\bar{i}}^{\dagger}(t) d_{\bar{i}}(t) c_{\mathbf{k} \sigma \eta}(t) d_{j}^{\dagger}\left(t^{\prime}\right)\right\rangle & =\left\langle d_{\bar{i}}^{\dagger} d_{\bar{i}}\right\rangle\left\langle T c_{\mathbf{k} \sigma \eta}(t) d_{j}^{\dagger}\left(t^{\prime}\right)\right\rangle
\end{aligned}
$$

onde desprezamos a média que envolve dois operadores destruição do tipo $d$ no mesmo instante de tempo. Com isso a equação de movimento para $G_{i j}^{(2)}$ fica

$$
\begin{aligned}
\left(i \frac{\partial}{\partial t}-\epsilon_{i}-U\right) G_{i j}^{(2)}\left(t, t^{\prime}\right) & =\delta\left(t-t^{\prime}\right)\left[\delta_{i j}\left\langle n_{\bar{i}}\right\rangle-\delta_{\bar{i} j}\left\langle d_{\bar{i}}^{\dagger} d_{i}\right\rangle\right] \\
& +\sum_{\mathbf{k} \sigma \eta} \frac{(-1)^{i \delta_{\sigma \downarrow}}}{\sqrt{2}} t_{\mathbf{k} \sigma}^{*}\left\langle n_{\bar{i}}\right\rangle \Gamma_{\mathbf{k} \sigma \eta j}\left(t, t^{\prime}\right) \\
& -\sum_{\mathbf{k} \sigma \eta} \frac{(-1)^{\bar{i} \delta_{\sigma \downarrow}}}{\sqrt{2}} t_{\mathbf{k} \sigma}^{*}\left\langle d_{\bar{i}}^{\dagger} d_{i}\right\rangle \Gamma_{\mathbf{k} \sigma \eta j}\left(t, t^{\prime}\right),
\end{aligned}
$$

onde $\Gamma_{\mathbf{k} \sigma \eta j}\left(t, t^{\prime}\right)=-i\left\langle T c_{\mathbf{k} \sigma \eta}(t) d_{j}^{\dagger}\left(t^{\prime}\right)\right\rangle$. Calculando a equação de movimento para $\Gamma_{\mathbf{k} \sigma \eta j}\left(t, t^{\prime}\right)$ obtemos

$$
\Gamma_{\mathbf{k} \sigma \eta j}\left(t, t^{\prime}\right)=\sum_{i_{1}} \frac{(-1)^{i_{1} \delta_{\sigma \downarrow}}}{\sqrt{2}} t_{\mathbf{k} \sigma} \int d t_{1} g_{\mathbf{k} \sigma \eta}\left(t, t_{1}\right) G_{i_{1} j}\left(t_{1}, t^{\prime}\right) .
$$


Substituindo (3.53) em (3.52) vem

$$
\begin{aligned}
\left(i \frac{\partial}{\partial t}-\epsilon_{i}-U\right) G_{i j}^{(2)}\left(t, t^{\prime}\right) & =\delta\left(t-t^{\prime}\right)\left[\delta_{i j}\left\langle n_{\bar{i}}\right\rangle-\delta_{\bar{i} j}\left\langle d_{\bar{i}}^{\dagger} d_{i}\right\rangle\right] \\
& +\left\langle n_{\bar{i}}\right\rangle \sum_{\mathbf{k} \sigma \eta i_{1}} \frac{(-1)^{\left(i+i_{1}\right) \delta_{\sigma \downarrow}}}{2}\left|t_{\mathbf{k} \sigma}\right|^{2} \int d t_{1} g_{\mathbf{k} \sigma \eta}\left(t, t_{1}\right) G_{i_{1} j}\left(t_{1}, t^{\prime}\right) \\
& -\left\langle d_{\bar{i}}^{\dagger} d_{i}\right\rangle \sum_{\mathbf{k} \sigma \eta i_{1}} \frac{(-1)^{\left(\bar{i}+i_{1}\right) \delta_{\sigma \downarrow}}}{2}\left|t_{\mathbf{k} \sigma}\right|^{2} \int d t_{1} g_{\mathbf{k} \sigma \eta}\left(t, t_{1}\right) G_{i_{1} j}\left(t_{1}, t^{\prime}\right),
\end{aligned}
$$

que pode ser escrita da seguinte forma

$$
\begin{aligned}
\left(i \frac{\partial}{\partial t}-\epsilon_{i}-U\right) G_{i j}^{(2)}\left(t, t^{\prime}\right) & =\delta\left(t-t^{\prime}\right)\left[\delta_{i j}\left\langle n_{\bar{i}}\right\rangle-\delta_{\bar{i} j}\left\langle d_{\bar{i}}^{\dagger} d_{i}\right\rangle\right] \\
& +\sum_{i_{1}} \int d t_{1}\left[\left\langle n_{\bar{i}}\right\rangle \Sigma_{i i_{1}}\left(t, t_{1}\right)-\left\langle d_{\bar{i}}^{\dagger} d_{i}\right\rangle \Sigma_{\bar{i} i_{1}}\left(t, t_{1}\right)\right] G_{i_{1} j}\left(t_{1}, t^{\prime}\right)
\end{aligned}
$$

quando utilizamos a definição (3.50). Além disso, definindo a função de Green $G_{i j}^{0(2)}$ tal que

$$
\left(i \frac{\partial}{\partial t}-\epsilon_{i}-U\right) G_{i j}^{0(2)}\left(t, t^{\prime}\right)=\delta_{i j} \delta\left(t-t^{\prime}\right)\left\langle n_{\bar{i}}\right\rangle-\delta_{\bar{i} j} \delta\left(t-t^{\prime}\right)\left\langle d_{\bar{i}}^{\dagger} d_{i}\right\rangle,
$$

a Eq.(3.55) pode ser reescrita assim

$$
G_{i j}^{(2)}\left(t, t^{\prime}\right)=G_{i j}^{0(2)}\left(t, t^{\prime}\right)+\sum_{i_{1} i_{2}} \iint d t_{1} d t_{2} G_{i i_{2}}^{0(2)}\left(t, t_{2}\right) \Sigma_{i_{2} i_{1}}\left(t_{2}, t_{1}\right) G_{i_{1} j}\left(t_{1}, t^{\prime}\right) .
$$

Substituindo a Eq.(3.57) na Eq.(3.49) encontramos

$$
\begin{aligned}
\left(i \frac{\partial}{\partial t}-\epsilon_{i}\right) G_{i j}\left(t, t^{\prime}\right) & =\delta_{i j} \delta\left(t-t^{\prime}\right)+U G_{i j}^{0(2)}\left(t, t^{\prime}\right) \\
& +\sum_{i_{1}} \int d t_{1} \Sigma_{i i_{1}}\left(t, t_{1}\right) G_{i_{1} j}\left(t_{1}, t^{\prime}\right) \\
& +U \sum_{i_{1} i_{2}} \iint d t_{1} d t_{2} G_{i i_{2}}^{0(2)}\left(t, t_{2}\right) \Sigma_{i_{2} i_{1}}\left(t_{2}, t_{1}\right) G_{i_{1} j}\left(t_{1}, t^{\prime}\right)
\end{aligned}
$$

$\mathrm{Ou}$

$$
\begin{aligned}
G_{i j}\left(t, t^{\prime}\right) & =G_{i j}^{0}\left(t, t^{\prime}\right)+\sum_{i_{1}} \int d t_{1} G_{i i_{1}}^{0}\left(t, t_{1}\right) U G_{i_{1} j}^{0(2)}\left(t_{1}, t^{\prime}\right) \\
& +\sum_{i_{1} i_{2}} \int d t_{1} d t_{2} G_{i i_{2}}^{0}\left(t, t_{2}\right) \Sigma_{i_{2} i_{1}}\left(t_{2}, t_{1}\right) G_{i_{1} j}\left(t_{1}, t^{\prime}\right) \\
& +\sum_{i_{1} i_{2} i_{3}} \iiint d t_{1} d t_{2} d t_{3} G_{i i_{3}}^{0}\left(t, t_{3}\right) U G_{i_{3} i_{2}}^{0(2)}\left(t_{3}, t_{2}\right) \Sigma_{i_{2} i_{1}}\left(t_{2}, t_{1}\right) G_{i_{1} j}\left(t_{1} t^{\prime}\right),
\end{aligned}
$$

onde $G_{i j}^{0}$ é a função de Green do ponto quântico isolado e não-interagente, i.e.,

$$
\left(i \frac{\partial}{\partial t}-\epsilon_{i}\right) G_{i j}^{0}\left(t, t^{\prime}\right)=\delta_{i j} \delta\left(t-t^{\prime}\right)
$$


Para se obter a equação de Dyson para a função de Green Eq.(3.59) definimos

$$
\widetilde{G}_{i j}^{0}\left(t, t^{\prime}\right)=G_{i j}^{0}\left(t, t^{\prime}\right)+\sum_{i_{1}} \int d t_{1} G_{i i_{1}}^{0}\left(t, t_{1}\right) U G_{i_{1 j}}^{0(2)}\left(t_{1}, t^{\prime}\right)
$$

de modo que a Eq.(3.59) fica

$$
G_{i j}\left(t, t^{\prime}\right)=\widetilde{G}_{i j}^{0}\left(t, t^{\prime}\right)+\sum_{i_{1} i_{2}} \iint d t_{1} d t_{2} \widetilde{G}_{i i_{2}}^{0}\left(t, t_{2}\right) \sum_{i_{2} i_{1}}\left(t_{2}, t_{1}\right) G_{i_{1 j} j}\left(t_{1}, t^{\prime}\right) .
$$

Usando a analogia formal entre funções de Green de equilíbrio e de não-equilíbrio, reescrevemos (3.62) com tempos $\tau$ e $\tau^{\prime}$ definidos ao longo do contorno,

$$
G_{i j}\left(\tau, \tau^{\prime}\right)=\widetilde{G}_{i j}^{0}\left(\tau, \tau^{\prime}\right)+\sum_{i_{1} i_{2}} \iint d \tau_{1} d \tau_{2} \widetilde{G}_{i i_{2}}^{0}\left(\tau, \tau_{2}\right) \Sigma_{i_{2} i_{1}}\left(\tau_{2}, \tau_{1}\right) G_{i_{1} j}\left(\tau_{1}, \tau^{\prime}\right)
$$

Aplicando as regras de Langreth, sumarizadas no Cap. 2, obtemos o seguinte sistema de equações acopladas

$$
\begin{aligned}
\mathbf{G}^{r}\left(t, t^{\prime}\right) & =\widetilde{\mathbf{G}}^{0 r}\left(t, t^{\prime}\right)+\iint d t_{1} d t_{2} \widetilde{\mathbf{G}}^{0 r}\left(t, t_{1}\right) \Sigma^{r}\left(t_{1}, t_{2}\right) \mathbf{G}^{r}\left(t_{2}, t^{\prime}\right) \\
\mathbf{G}^{a}\left(t, t^{\prime}\right) & =\widetilde{\mathbf{G}}^{0 a}\left(t, t^{\prime}\right)+\iint d t_{1} d t_{2} \widetilde{\mathbf{G}}^{0 a}\left(t, t_{1}\right) \Sigma^{a}\left(t_{1}, t_{2}\right) \mathbf{G}^{a}\left(t_{2}, t^{\prime}\right) \\
\mathbf{G}^{<}\left(t, t^{\prime}\right) & =\iiint \int d t_{1} d t_{2} d t_{3} d t_{4}\left[\delta\left(t-t_{1}\right) \delta\left(t_{1}-t_{2}\right)+\mathbf{G}^{r}\left(t, t_{1}\right) \Sigma^{r}\left(t_{1}, t_{2}\right)\right] \widetilde{\mathbf{G}}^{0<}\left(t_{2}, t_{3}\right) \\
& \times\left[\delta\left(t_{3}-t_{4}\right) \delta\left(t_{4}-t^{\prime}\right)+\Sigma^{a}\left(t_{3}, t_{4}\right) \mathbf{G}^{a}\left(t_{4}, t^{\prime}\right)\right] \\
& +\iint d t_{1} d t_{2} \mathbf{G}^{r}\left(t, t_{1}\right) \Sigma^{<}\left(t_{1}, t_{2}\right) \mathbf{G}^{a}\left(t_{2}, t^{\prime}\right),
\end{aligned}
$$

onde as funções de Green foram denotadas matricialmente. Para um Hamiltoniano independente do tempo as funções de Green variam com $t-t^{\prime}$. Logo a transformada de Fourier leva às seguintes equações

$$
\begin{aligned}
\mathbf{G}^{r}(\omega) & =\widetilde{\mathbf{G}}^{0 r}(\omega)+\widetilde{\mathbf{G}}^{0 r}(\omega) \Sigma^{r}(\omega) \mathbf{G}^{r}(\omega) \\
\mathbf{G}^{a}(\omega) & =\widetilde{\mathbf{G}}^{0 a}(\omega)+\widetilde{\mathbf{G}}^{0 a}(\omega) \Sigma^{a}(\omega) \mathbf{G}^{a}(\omega) \\
\mathbf{G}^{<}(\omega) & =\mathbf{G}^{r}(\omega) \Sigma^{<}(\omega) \mathbf{G}^{a}(\omega),
\end{aligned}
$$

onde

$$
\begin{aligned}
\widetilde{\mathbf{G}}^{0 r} & =\left(\begin{array}{cc}
\frac{1}{\omega-\epsilon_{1}} & 0 \\
0 & \frac{1}{\omega-\epsilon_{2}}
\end{array}\right)+\left(\begin{array}{cc}
\frac{1}{\omega-\epsilon_{1}} & 0 \\
0 & \frac{1}{\omega-\epsilon_{2}}
\end{array}\right) U\left(\begin{array}{cc}
\frac{\left\langle\widehat{n}_{2}\right\rangle}{\omega-\epsilon_{1}-U} & -\frac{\left\langle\widehat{n}_{21}\right\rangle}{\omega-\epsilon_{1}-U} \\
-\frac{\left\langle\hat{n}_{12}\right\rangle}{\omega-\epsilon_{2}-U} & \frac{\left\langle\hat{n}_{1}\right\rangle}{\omega-\epsilon_{2}-U}
\end{array}\right) \\
& =\left(\begin{array}{cc}
\frac{\omega-\epsilon_{1}-U\left(1-\left\langle\widehat{n}_{2}\right\rangle\right)}{\left(\omega-\epsilon_{1}\right)\left(\omega-\epsilon_{1}-U\right)} & \frac{-\left\langle\widehat{n}_{21}\right\rangle U}{\left(\omega-\epsilon_{1}\right)\left(\omega-\epsilon_{1}-U\right)} \\
\frac{-U\left\langle\hat{n}_{12}\right\rangle}{\left(\omega-\epsilon_{2}\right)\left(\omega-\epsilon_{2}-U\right)} & \frac{\omega-\epsilon_{2}-U\left(1-\left\langle\hat{n}_{1}\right\rangle\right)}{\left(\omega-\epsilon_{2}\right)\left(\omega-\epsilon_{2}-U\right)}
\end{array}\right),
\end{aligned}
$$

sendo $\left\langle\widehat{n}_{i}\right\rangle=\left\langle d_{i}^{\dagger} d_{i}\right\rangle$, e $\left\langle\widehat{n}_{i j}\right\rangle=\left\langle d_{j}^{\dagger} d_{i}\right\rangle$. 
Para calcularmos $\mathbf{G}^{>}$utilizamos a relação $\mathbf{G}^{>}-\mathbf{G}^{<}=\mathbf{G}^{r}-\mathbf{G}^{a}$, e para as auto-energias temos

$$
\begin{aligned}
\Sigma_{j i}^{r, a}(\epsilon) & =\frac{1}{2} \sum_{\mathbf{k} \sigma \eta}\left|t_{\mathbf{k} \sigma}\right|^{2}(-1)^{(i+j) \delta_{\sigma \downarrow}} g_{\mathbf{k} \sigma \eta}^{r, a}(\epsilon) \\
& =\frac{1}{2} \sum_{\mathbf{k} \sigma \eta}\left|t_{\mathbf{k} \sigma}\right|^{2}(-1)^{(i+j) \delta_{\sigma \downarrow}}\left[P \frac{1}{\epsilon-\epsilon_{\mathbf{k} \sigma \eta}} \mp \pi i \delta\left(\epsilon-\epsilon_{\mathbf{k} \sigma \eta}\right)\right] \\
& =\Lambda_{i j}(\epsilon) \mp \frac{i}{2} \Gamma_{i j}(\epsilon),
\end{aligned}
$$

onde $\Lambda_{i j}(\epsilon)=\frac{1}{2} \sum_{\mathbf{k} \sigma \eta}\left|t_{\mathbf{k} \sigma}\right|^{2}(-1)^{(i+j) \delta_{\sigma \downarrow}} P \frac{1}{\epsilon-\epsilon_{\mathbf{k} \sigma \eta}}$, e $\Gamma_{i j}(\epsilon)=\frac{1}{2} 2 \pi \sum_{\mathbf{k} \sigma \eta}\left|t_{\mathbf{k} \sigma}\right|^{2}(-1)^{(i+j) \delta_{\sigma \downarrow}}$ $\delta\left(\epsilon-\epsilon_{\mathbf{k} \sigma \eta}\right)$. Considerando a aproximação de banda larga (wide-band limit), proposta por Jauho, Wingreen e Meir [7], temos que $\Lambda_{i j}(\epsilon)=0$ e $\Gamma_{i j}(\epsilon)=\Gamma_{i j}=$ cte. Nessa aproximação a densidade de estados dos terminais, $\rho\left(\epsilon_{\mathbf{k} j \sigma}\right)$, é considerada constante e o acoplamento $t_{\mathbf{k} \sigma}$ independe do vetor de onda $\mathbf{k}$. Com isso a matriz de auto-energia fica

$$
\begin{aligned}
\boldsymbol{\Sigma}^{r} & =-\frac{i}{2}\left(\begin{array}{cc}
\Gamma_{11} & \Gamma_{12} \\
\Gamma_{21} & \Gamma_{22}
\end{array}\right)=-\frac{i}{2}\left(\begin{array}{cc}
\Gamma_{11}^{L} & \Gamma_{12}^{L} \\
\Gamma_{21}^{L} & \Gamma_{22}^{L}
\end{array}\right)-\frac{i}{2}\left(\begin{array}{cc}
\Gamma_{11}^{R} & \Gamma_{12}^{R} \\
\Gamma_{21}^{R} & \Gamma_{22}^{R}
\end{array}\right), \\
& =-\frac{i}{4}\left(\begin{array}{cc}
\Gamma_{\uparrow}^{L}+\Gamma_{\downarrow}^{L} & \Gamma_{\uparrow}^{L}-\Gamma_{\downarrow}^{L} \\
\Gamma_{\uparrow}^{L}-\Gamma_{\downarrow}^{L} & \Gamma_{\uparrow}^{L}+\Gamma_{\downarrow}^{L}
\end{array}\right)-\frac{i}{4}\left(\begin{array}{cc}
\Gamma_{\uparrow}^{R}+\Gamma_{\downarrow}^{R} & \Gamma_{\uparrow}^{R}-\Gamma_{\downarrow}^{R} \\
\Gamma_{\uparrow}^{R}-\Gamma_{\downarrow}^{R} & \Gamma_{\uparrow}^{R}+\Gamma_{\downarrow}^{R}
\end{array}\right),
\end{aligned}
$$

onde $\Gamma_{\sigma}^{\eta}=2 \pi \sum_{\mathbf{k}}\left|t_{\mathbf{k} \sigma}\right|^{2} \delta\left(\epsilon-\epsilon_{\mathbf{k} \sigma \eta}\right)$. A auto-energia $\boldsymbol{\Sigma}^{r}$ dá origem ao alargamento dos níveis $\epsilon_{1}$ e $\epsilon_{2}$ do ponto quântico, visto que essa introduz o acoplamento entre esses níveis localizados e as bandas de condução dos terminais ferromagnéticos. Para $\boldsymbol{\Sigma}^{a}$ temos simplesmente $\boldsymbol{\Sigma}^{a}=\boldsymbol{\Sigma}^{r^{*}}$, e para $\boldsymbol{\Sigma}^{<}$obtemos

$$
\begin{aligned}
\boldsymbol{\Sigma}^{<} & =i n_{L}\left(\begin{array}{cc}
\Gamma_{11}^{L} & \Gamma_{12}^{L} \\
\Gamma_{21}^{L} & \Gamma_{22}^{L}
\end{array}\right)+i n_{R}\left(\begin{array}{cc}
\Gamma_{11}^{R} & \Gamma_{12}^{R} \\
\Gamma_{21}^{R} & \Gamma_{22}^{R}
\end{array}\right) \\
& =\frac{i}{2} n_{L}\left(\begin{array}{cc}
\Gamma_{\uparrow}^{L}+\Gamma_{\downarrow}^{L} & \Gamma_{\uparrow}^{L}-\Gamma_{\downarrow}^{L} \\
\Gamma_{\uparrow}^{L}-\Gamma_{\downarrow}^{L} & \Gamma_{\uparrow}^{L}+\Gamma_{\downarrow}^{L}
\end{array}\right)+\frac{i}{2} n_{R}\left(\begin{array}{cc}
\Gamma_{\uparrow}^{R}+\Gamma_{\downarrow}^{R} & \Gamma_{\uparrow}^{R}-\Gamma_{\downarrow}^{R} \\
\Gamma_{\uparrow}^{R}-\Gamma_{\downarrow}^{R} & \Gamma_{\uparrow}^{R}+\Gamma_{\downarrow}^{R}
\end{array}\right)
\end{aligned}
$$

As auto-energias (3.70) e (3.71) são substituídas nas Eqs.(3.65)-(3.67) de modo a obtermos as funções de Green.

A formulação desenvolvida nesse capítulo será a base de cálculo para o próximo capítulo, em que aplicaremos as equações (3.24) e (3.43) para calcular corrente e ruído em um sistema de ponto quântico acoplado a eletrodos ferromagnéticos. 


\section{Capítulo 4}

\section{Resultados para o sistema FM-PQ-FM}

Nesse capítulo apresentamos os resultados de transporte obtidos para o sistema de um ponto quântico acoplado, via barreiras de tunelamento, a contatos ferromagnéticos (FM-PQ-FM). Os cálculos foram feitos utilizando-se a formulação desenvolvida no capítulo anterior. Mais especificamente, estudamos a função espectral $A(\epsilon)=i \operatorname{Tr}\left[\mathbf{G}^{r}(\epsilon)-\mathbf{G}^{a}(\epsilon)\right]$, as ocupações eletrônicas $n_{\uparrow}$ e $n_{\downarrow}$ do ponto quântico, as correntes $I_{\uparrow}$ e $I_{\downarrow}$ e os ruídos $S_{\uparrow}$ e $S_{\downarrow}$. Observamos que a função espectral do ponto quântico depende do alinhamento magnético e da polarização dos terminais. Em particular, quando temos $100 \%$ de polarização no alinhamento paralelo, observamos a formação de um pico estreito na função espectral. Isso está relacionado ao fato do estado de spin oposto a magnetização dos eletrodos se desacoplarem dos terminais. Com relação às ocupações, encontramos um acúmulo de spin no ponto quântico e portanto uma magnetização $m$ não nula. Mostramos que $m$ pode ser ajustado em intensidade e sinal através da tensão externa $V$ e também através do alinhamento magnético dos eletrodos. Por exemplo, podemos flipar a polarização do ponto quântico variando o alinhamento magnético dos eletrodos de $\mathrm{P}$ para AP.

A magnetização não nula do ponto quântico resulta num bloqueio de Coulomb dependente de spin. Esse efeito nos permite controlar o sinal e a intensidade da polarização da corrente através de uma tensão externa. Os efeitos do bloqueio de Coulomb dependente de spin sobre o ruído também foram analisados. Discutimos também os efeitos do espalhamento de spin sobre a corrente e o ruído. Encontramos que, enquanto a corrente na configuração AP varia monotonicamente com $R$ (parâmetro de espalhamento de spin), o ruído varia não monotônicamente, sendo suprimido e depois intensificado como função de $R$. Através de uma analogia entre o sistema FM-PQ-FM e um sistema de tripla-barreira de tunelamento, damos uma interpretação física para a supressão do ruído devido a $R$. Além desses efeitos, uma série de outros resultados estão ilustrados ao longo do capítulo. 


\subsection{Procedimento Numérico}

Como vimos no capítulo anterior, para se obter a corrente e o ruído devemos calcular as várias funções de Green do ponto quântico $\mathbf{G}^{r}, \mathbf{G}^{a}, \mathbf{G}^{>}$e $\mathbf{G}^{<}$. Para isso, precisamos especificar os valores de $\epsilon_{1}, \epsilon_{2}, U, \Gamma_{\sigma}^{\eta}$ e $\left\langle\widehat{n}_{1}\right\rangle,\left\langle\widehat{n}_{2}\right\rangle,\left\langle\widehat{n}_{12}\right\rangle$ e $\left\langle\widehat{n}_{21}\right\rangle$. Os níveis $\epsilon_{1}$ e $\epsilon_{2}$ são dados por $\epsilon_{i}=\epsilon_{d}+(-1)^{i} R$, onde $\epsilon_{d}=\epsilon_{0}-e V / 2$, sendo $e$ a carga do elétron em módulo, $V$ a tensão externa aplicada entre eletrodos, e $\epsilon_{0}$ o nível do ponto quântico na ausência de tensão $(V=0)$. Em nosso modelo adotamos $\epsilon_{0}=0.5$ meV, de modo que o nível do ponto quântico está acima dos potenciais químicos do emissor $\mu_{L}$ e do coletor $\mu_{R}$ (adotamos $\mu_{L}=0$ e $\mu_{R}=\mu_{L}-e V$ ), quando a voltagem é nula. A tensão $\mathrm{eV}$ varia tipicamente entre 0 e $5 \mathrm{meV}$, que é uma faixa de valores suficiente para observarmos os efeitos físicos relevantes. Os valores dos parâmetros $R$ e $U$ serão especificados no decorrer do texto. Descrevemos o magnetismo dos eletrodos através dos parâmetros dependentes de spin $\Gamma_{\sigma}^{\eta}$, que são dados através das equações [80],

$$
\begin{aligned}
\Gamma_{\sigma}^{L} & =\Gamma_{0}^{L}\left[1+(-1)^{\delta_{\sigma \downarrow}} p_{L}\right] \\
\Gamma_{\sigma}^{R} & =\Gamma_{0}^{R}\left[1 \pm(-1)^{\delta_{\sigma \downarrow}} p_{R}\right],
\end{aligned}
$$

onde $L(R)$ é o índice para o terminal da esquerda (direita). Os parâmetros $\Gamma_{0}^{L}$ e $\Gamma_{0}^{R}$ dão as intensidades dos acoplamentos entre ponto-quântico e eletrodos, e $p_{L}$ e $p_{R}$ fornecem a polarização desses eletrodos. Os sinais \pm que aparecem em $\Gamma_{\sigma}^{R}$ correspondem ao alinhamento magnético paralelo $(+)$ e anti-paralelo $(-)$ entre os reservatórios. Essa mudança de sinal se dá somente em $\Gamma_{\sigma}^{R}$, pois assumimos que o terminal da esquerda (L) tem uma magnetização fixa (hard side), enquanto o da direita $(\mathrm{R})$ tem uma magnetização variável entre $\uparrow$ e $\downarrow$ (soft side). Além dos sinais \pm em $\Gamma_{\sigma}^{R}$, temos que o fator $(-1)^{\delta_{\sigma \downarrow}}$ vale +1 para spin $\uparrow$ e -1 para spin $\downarrow$. Isso nos fornece as populações de spin majoritária e minoritária nos terminais. Por exemplo, para $p_{L}=1$ temos $\Gamma_{\uparrow}^{L}=2 \Gamma_{0}^{L}$ e $\Gamma_{\downarrow}^{L}=0$, logo o eletrodo da esquerda é $100 \%$ polarizado com spin $\uparrow$. Se $p_{L}<1$ teremos ambas as populações de spin, porém a população $\uparrow$ será majoritária, o que irá gerar $\Gamma_{\uparrow}^{L}>\Gamma_{\downarrow}^{L}$. Para $p_{R}=1$ temos $\Gamma_{\uparrow}^{R}=2 \Gamma_{0}^{R}$ e $\Gamma_{\downarrow}^{R}=0$ se o sinal de + for utilizado e o contrário, $\Gamma_{\uparrow}^{R}=0$ e $\Gamma_{\downarrow}^{R}=2 \Gamma_{0}^{R}$, se adotarmos o sinal negativo. Na maioria dos nossos resultados consideramos $\Gamma_{0}^{L}=\Gamma_{0}^{R}=\Gamma_{0}=10 \mu \mathrm{eV}$ [81], porém, quando analisamos o ruído em sistemas fortemente assimétricos, adotamos $\Gamma_{0}^{R}=100 \Gamma_{0}^{L}$.

O cálculo dos valores médios $\left\langle\widehat{n}_{1}\right\rangle,\left\langle\widehat{n}_{2}\right\rangle,\left\langle\widehat{n}_{12}\right\rangle$ e $\left\langle\widehat{n}_{21}\right\rangle$ são feitos através da função de Green menor $\mathbf{G}^{<}$, visto que

$$
\left\langle\widehat{n}_{i j}\right\rangle=\left\langle d_{i}^{\dagger} d_{j}\right\rangle=-i G_{j i}^{<}(t, t)=-i \int \frac{d \epsilon}{2 \pi} G_{j i}^{<}(\epsilon) .
$$


Com isso temos as seguintes equações

$$
\begin{array}{lll}
n_{1}=\left\langle\widehat{n}_{1}\right\rangle=\int \frac{d \epsilon}{2 \pi i} G_{11}^{<}(\epsilon), & n_{12}=\left\langle\widehat{n}_{12}\right\rangle=\int \frac{d \epsilon}{2 \pi i} G_{21}^{<}(\epsilon), \\
n_{2}=\left\langle\widehat{n}_{2}\right\rangle=\int \frac{d \epsilon}{2 \pi i} G_{22}^{<}(\epsilon), & n_{21}=\left\langle\widehat{n}_{21}\right\rangle=\int \frac{d \epsilon}{2 \pi i} G_{12}^{<}(\epsilon) .
\end{array}
$$

Por outro lado, a função de Green $\mathbf{G}^{<}$depende das médias $\left\langle\widehat{n}_{1}\right\rangle,\left\langle\widehat{n}_{2}\right\rangle,\left\langle\widehat{n}_{12}\right\rangle$ e $\left\langle\widehat{n}_{21}\right\rangle$ [veja Eqs.(3.65)-(3.68)]. Desse modo, precisamos efetuar um cálculo autoconsistente, para obtermos $\mathbf{G}^{<}$e as ocupações $\left\langle\widehat{n}_{i j}\right\rangle$. O procedimento numérico está ilustrado na Fig.(4.1). Iniciamos o cálculo definindo os parâmetros e atribuindo valores para as médias $n_{1}, n_{2}, n_{12}$ e $n_{21}$. Com esse valores calculamos as funções de Green do ponto quântico $\mathbf{G}^{r}, \mathbf{G}^{a}, \mathbf{G}^{<}$e $\mathbf{G}^{>}$. Em seguida utilizamos essas funções de Green para calcular os novos valores de $n_{1}, n_{2}, n_{12}$ e $n_{21}$. Repetimos esse procedimento até a convergência dos resultados. Por último, calculamos grandezas de interesse como a função espectral, ocupação eletrônica, corrente e ruído, que estão discutidos nas seções seguintes.

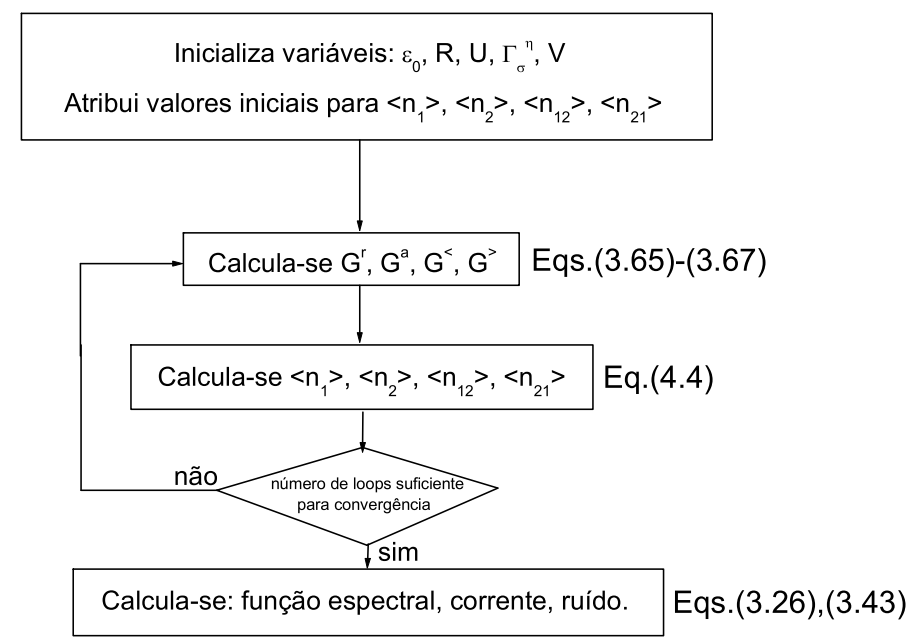

Figura 4.1: Esquema do procedimento numérico utilizado no cálculo auto-consistente das funções de Green $\mathbf{G}^{r}, \mathbf{G}^{a}, \mathbf{G}^{<}$e $\mathbf{G}^{>}$e das médias $\left\langle n_{1}\right\rangle,\left\langle n_{2}\right\rangle,\left\langle n_{12}\right\rangle$ e $\left\langle n_{21}\right\rangle$. O cálculo auto-consistente converge em média com seis iterações.

\subsection{Função Espectral}

Uma quantidade relevante em transporte é a função espectral $A(\epsilon)=i \operatorname{Tr}\left[\mathbf{G}^{r}(\epsilon)-\right.$ $\left.\mathbf{G}^{a}(\epsilon)\right]$, que nos fornece a densidade de estados do ponto quântico e as energias necessárias para excitar elétrons dos terminais para o ponto quântico. Note que $A(\epsilon)$ aparece em alguns termos de (3.24) e (3.43), indicando a dependência da corrente e do ruído com os estados do ponto quântico. Em particular, no caso 
não-interagente $(U=0)$ e espalhamento de spin nulo $(R=0)$, a função de Green $\mathbf{G}^{r}(\epsilon)$ assume a seguinte forma

$$
\mathbf{G}^{r}(\epsilon)=\left(\begin{array}{cc}
\frac{1}{\epsilon-\epsilon_{\uparrow}+i \Gamma_{\uparrow} / 2} & 0 \\
0 & \frac{1}{\epsilon-\epsilon_{\downarrow}+i \Gamma_{\downarrow} / 2}
\end{array}\right) .
$$

Com isso a função espectral $A(\epsilon)$ é simplesmente uma soma de lorentzianas centradas em $\epsilon_{\uparrow}$ e $\epsilon_{\downarrow}$ e de larguras $\Gamma_{\uparrow}=\Gamma_{\uparrow}^{L}+\Gamma_{\uparrow}^{R}$ e $\Gamma_{\downarrow}=\Gamma_{\downarrow}^{L}+\Gamma_{\downarrow}^{R}$, isto é,

$$
A(\epsilon)=\frac{\Gamma_{\uparrow}}{\left(\epsilon-\epsilon_{\uparrow}\right)^{2}+\left(\frac{\Gamma_{\uparrow}}{2}\right)^{2}}+\frac{\Gamma_{\downarrow}}{\left(\epsilon-\epsilon_{\downarrow}\right)^{2}+\left(\frac{\Gamma_{\downarrow}}{2}\right)^{2}} .
$$

A partir dessa expressão para $A(\epsilon)$, observamos que o magnetismo dos terminais afeta $A(\epsilon)$ através da largura de linha $\Gamma_{\sigma}$. Quanto menor a largura de linha $\Gamma_{\sigma}$, menos o estado $\epsilon_{\sigma}$ está acoplado aos terminais. No caso limite $\Gamma_{\sigma} \rightarrow 0$ temos um estado $\epsilon_{\sigma}$ totalmente desacoplado, e por isso surge uma função delta correspondente ao estado $\epsilon_{\sigma}$, conforme veremos nas próximas figuras. Também analisaremos os efeitos da interação de Coulomb e do espalhamento de spin sobre $A(\epsilon)$.

\subsubsection{Efeitos do ferromagnetismo dos eletrodos sobre a função espectral}

Comecemos avaliando os efeitos dos eletrodos ferromagnéticos sobre a função espectral. Na figura (4.2) mostramos $A(\epsilon)$ para diferentes parâmetros de polarização $p_{L}$ e $p_{R}\left(p_{L}=p_{R}=p\right)$, nas configurações paralela (a) e anti-paralela (b), mantendo $U=0$ e $V=0$. Para $p=0$ as configurações paralela (P) e anti-paralela (AP) são idênticas, visto que não há polarização nos terminais. O pico centrado em 0.5 corresponde aos níveis degenerados $\epsilon_{\uparrow}$ e $\epsilon_{\downarrow}$. Variando $p$ de 0 (caso não-magnético) a 1 (caso $100 \%$ polarizado) notamos que $A(\epsilon)$ apresenta comportamentos contrastantes entre os casos P e AP. Por exemplo, no caso paralelo, o aumento da polarização leva ao aparecimento de um pico estreito sobre uma banda larga na função espectral. Já no caso anti-paralelo, $A(\epsilon)$ é insensível a variação de $p$ (as curvas para os vários $p$ 's estão superpostas).

Esse contraste entre as configurações $\mathrm{P}$ e AP deve-se aos valores que as taxas $\Gamma_{\sigma}^{L}$ e $\Gamma_{\sigma}^{R}$ assumem em cada caso. Por exemplo, a taxa de tunelamento para spin $\downarrow$, na configuração paralela, tende a zero em ambos os terminais quando $p \rightarrow 1$. Desse modo, o nível $\epsilon_{\downarrow}$ se desacopla dos contatos ferromagnéticos, resultando no pico estreito da Fig.4.2(a). Já na configuração AP os níveis degenerados $\epsilon_{\uparrow} \mathrm{e}$ $\epsilon_{\downarrow}$ permanecem acoplados a um dos terminais quando $p=1$. Note que nessa configuração a largura de linha para spin $\uparrow$ é $\Gamma_{\uparrow}^{L}+\Gamma_{\uparrow}^{R}=\Gamma_{0}(1+p)+\Gamma_{0}(1-p)=2 \Gamma_{0}$ e para spin $\downarrow$ temos $\Gamma_{\downarrow}^{L}+\Gamma_{\downarrow}^{R}=\Gamma_{0}(1-p)+\Gamma_{0}(1+p)=2 \Gamma_{0}$. Portanto ambas as 

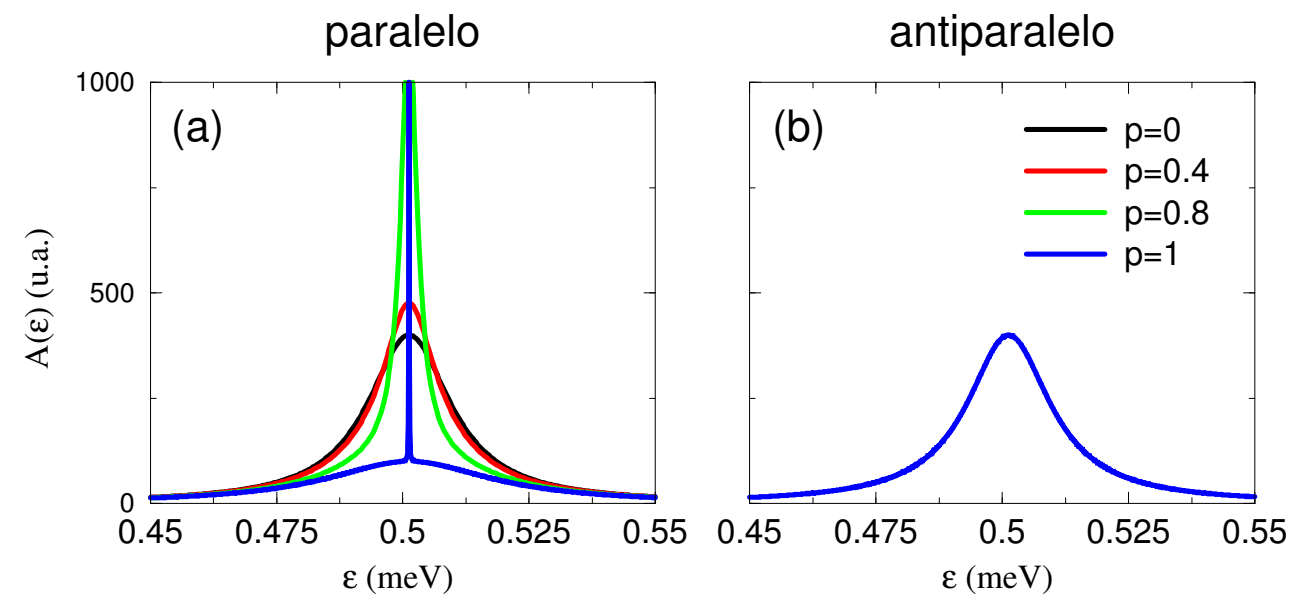

Figura 4.2: Função espectral $A(\epsilon)$ nas configurações (a) paralela (P) e (b) anti-paralela (AP) para diferentes valores de $p_{L}=p_{R}=p$. Enquanto $A(\epsilon)$ é insensível ao valor de $p$ na configuração AP, a função espectral varia apreciavelmente no caso paralelo, chegando a formar um pico estreito para $p=1$. Esse pico deve-se ao estado $\epsilon_{\downarrow}$ que se desacopla dos terminais no caso $100 \%$ polarizado. Adotamos aqui $U=0, R=0, \epsilon_{0}=0.5, V=0$.

larguras de linha não variam com $p$. Por isso a função espectral no alinhamento antiparalelo independe do valor de $p$, conforme vemos na Fig.4.2(b).

Na figura (4.3) mostramos a função espectral resolvida em spin $A_{\sigma}(\epsilon)$ para o alinhamento paralelo. A partir de $A_{\uparrow}(\epsilon)$ [Fig.4.3(a)] vemos que o aumento da polarização dos eletrodos leva a um alargamento do nível $\epsilon_{\uparrow}$. Tal alargamento deve-se ao aumento do parâmetro $\Gamma_{\uparrow}^{\eta}=\Gamma_{0}^{\eta}(1+p)$, o que tende a aumentar o acoplamento entre $\epsilon_{\uparrow}$ e os eletrodos, e conseqüentemente a sua largura de linha. Já para $A_{\downarrow}(\epsilon)$ [Fig.4.3(b)] vemos que o aumento de $p$ leva a uma diminuição da largura de linha de $\epsilon_{\downarrow}$. Isso decorre do fato de $\Gamma_{\downarrow}^{\eta}=\Gamma_{0}^{\eta}(1-p)$ tender a zero. Como na Fig.(4.2) temos a soma $A_{\uparrow}+A_{\downarrow}$, concluímos que o pico estreito observado na Fig.(4.2)(a) se deve a $A_{\downarrow}$, enquanto que a banda larga é proveniente de $A_{\uparrow}$. Vale salientar que, apesar de termos níveis degenerados $\epsilon_{\uparrow}=\epsilon_{\downarrow}$, os alargamentos de $\epsilon_{\uparrow}$ e $\epsilon_{\downarrow}$ diferem entre si devido a polarização dos terminais.

\subsubsection{Efeitos do espalhamento de spin sobre $A(\epsilon)$}

Analisemos agora os efeitos do espalhamento de spin sobre $A(\epsilon)$. Na figura (4.4) mostramos $A(\epsilon)$ para diferentes valores do parâmetro de espalhamento de spin $R$, mantendo $p_{L}$ e $p_{R}$ fixos $\left(p_{L}=p_{R}=1\right)$ e $U=0$. Para $R=0$ e configuração paralela [Fig.4.4(a)], temos um pico estreito sobre uma banda larga, como observado na Fig.4.2(a). À medida que $R$ aumenta, o pico estreito se alarga devido ao acoplamento indireto (provocado pelo espalhamento de spin) entre o nível $\epsilon_{\downarrow}$ e os terminais. Para $R$ suficientemente grande, surgem dois picos provenientes do desdobramento $\epsilon_{d} \rightarrow \epsilon_{d} \pm R$. Na configuração AP também temos o desdobramento 

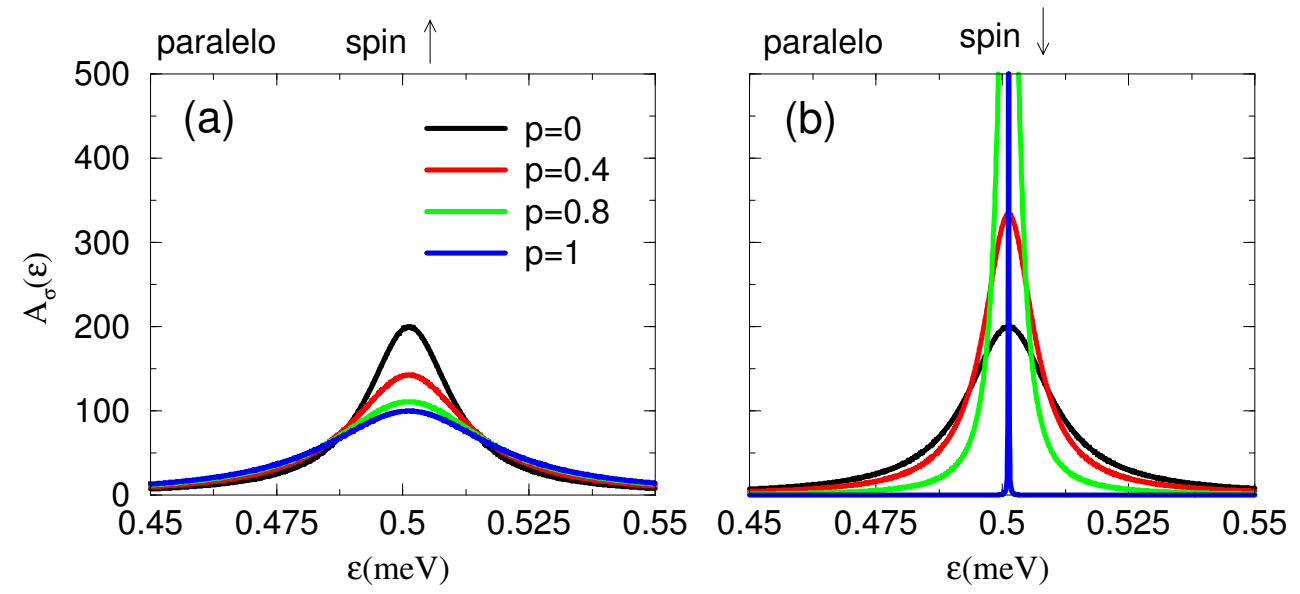

Figura 4.3: Função espectral resolvida em spin (a) $A_{\uparrow}(\epsilon)$ e (b) $A_{\downarrow}(\epsilon)$ para a configuração paralela. Enquanto $A_{\uparrow}$ se alarga com o aumento de $p, A_{\downarrow}$ torna-se cada vez mais estreito. $\mathrm{O}$ pico em $A_{\downarrow}$ origina o pico estreito observado na Fig.4.2(a).

do nível $\epsilon_{d}$ em $\epsilon_{d} \pm R$. Em particular, para $R=30 \mu e V$ a função espectral nas configurações $\mathrm{P}$ e AP se assemelham. Isso mostra que para $R$ suficientemente grande o alinhamento magnético dos terminais torna-se irrelevante para a função espectral. Veremos que a corrente e o ruído também independem do alinhamento magnético para $R$ suficientemente grande.
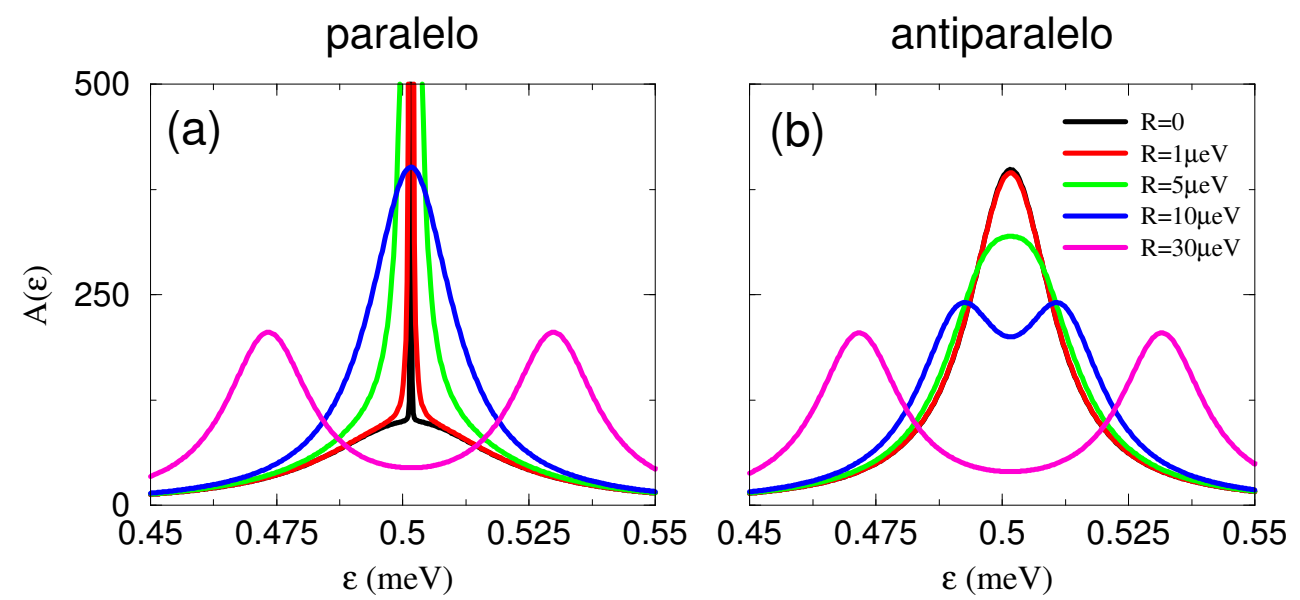

Figura 4.4: Função espectral nas configurações (a) paralela e (b) anti-paralela, $p_{L}=$ $p_{R}=1, U=0$, e diferentes valores do parâmetro de espalhamento de spin $R$. O pico estreito visto no caso $\mathrm{P}$ alarga-se com o aumento de $R$, devido ao acoplamento indireto entre $\epsilon_{\downarrow}$ e os contatos provocado pelo espalhamento de spin. Em ambos os alinhamentos magnéticos o pico $\epsilon_{d}$ desdobra-se em $\epsilon_{d}+R$ e $\epsilon_{d}-R$, e para $R$ suficientemente grande temos que $A(\epsilon)$ é o mesmo nos dois alinhamentos. 


\subsubsection{Efeitos da interação de Coulomb sobre a função es- pectral}

Vejamos agora os efeitos da interação de Coulomb sobre a função espectral. A figura (4.5) mostra $A(\epsilon)$ nas configurações P e AP, $R=30 \mu \mathrm{eV}, p=0.8$ e diferentes valores de $U$. Para $U=0$ temos somente dois picos: $\epsilon_{1}=\epsilon_{d}-R$ e $\epsilon_{2}=\epsilon_{d}+R$ como na Fig.4.4. Para $U \neq 0$, além dos picos $\epsilon_{1}$ e $\epsilon_{2}$, surgem picos em $\epsilon_{1}+U$ e $\epsilon_{2}+U$. Aumentando $U$, os picos $\epsilon_{1}+U$ e $\epsilon_{2}+U$ deslocam-se para a direita no gráfico. Quando os picos $\epsilon_{1}+U$ e $\epsilon_{2}$ se sobrepõem (para $U \approx \epsilon_{2}-\epsilon_{1}$ ) há a formação de um pico estreito na configuração paralela, semelhantemente ao da Fig.4.2(a) para $p=0.8$ e $R=0$. Note que na configuração AP, a sobreposição dos picos $\epsilon_{1}+U$ e $\epsilon_{2}$ não leva ao aparecimento de um pico estreito.

Como esse pico estreito é uma característica de $p$ grande (digamos $p=0.8$, $p=1$ ) e $R \rightarrow 0$, conforme vemos na Fig.4.4(a), temos um efeito bastante curioso na Fig.(4.5), onde observamos um pico estreito para $R$ relativamente grande $(R=30$ $\mu \mathrm{eV}$ ), e não para $R \rightarrow 0$ como era de se esperar. Isso sugere que quando os níveis $\epsilon_{1}+U$ e $\epsilon_{2}$ se cruzam, o sistema age como se o espalhamento de spin fosse suprimido, o que levaria ao aparecimento do pico estreito. Veremos posteriormente que os efeitos do cruzamento de $\epsilon_{1}+U$ e $\epsilon_{2}$ sobre o transporte podem ser descritos qualitativamente em termos de um coeficiente de transmissão que leva em conta um mecanismo de interferência entre os canais $\epsilon_{1}+U$ e $\epsilon_{2}$, e essa interferência tende a suprimir o transporte.

\subsection{Ocupação eletrônica no ponto quântico}

Nessa seção aplicamos o formalismo de funções de Green de não-equilíbrio para calcular a ocupação eletrônica do ponto quântico variando alguns parâmetros como a tensão $e V$, a interação de Coulomb $U$, o parâmetro de acoplamento $\Gamma_{0}^{R}$ e os parâmetros de polarização $p_{L}$ e $p_{R}$.

A ocupação eletrônica do ponto quântico é obtida através do cálculo autoconsistente discutido anteriormente e esquematizado na Fig.(4.1). Como as nossas funções de Green estão representados na base $\{1,2\}$, obtemos as médias $\left\langle d_{1}^{\dagger} d_{1}\right\rangle$, $\left\langle d_{2}^{\dagger} d_{2}\right\rangle,\left\langle d_{1}^{\dagger} d_{2}\right\rangle$ e $\left\langle d_{2}^{\dagger} d_{1}\right\rangle$ a partir das Eqs.(4.4). Para encontrarmos as ocupações resolvidas em spin $n_{\uparrow}=\left\langle d_{\uparrow}^{\dagger} d_{\uparrow}\right\rangle$ e $n_{\downarrow}=\left\langle d_{\downarrow}^{\dagger} d_{\downarrow}\right\rangle$, utilizamos as seguintes relações

$$
\begin{aligned}
& n_{\uparrow}=\left\langle d_{\uparrow}^{\dagger} d_{\uparrow}\right\rangle=\frac{1}{2}\left[\left\langle d_{1}^{\dagger} d_{1}\right\rangle+\left\langle d_{1}^{\dagger} d_{2}\right\rangle+\left\langle d_{2}^{\dagger} d_{1}\right\rangle+\left\langle d_{2}^{\dagger} d_{2}\right\rangle\right] \\
& n_{\downarrow}=\left\langle d_{\downarrow}^{\dagger} d_{\downarrow}\right\rangle=\frac{1}{2}\left[\left\langle d_{1}^{\dagger} d_{1}\right\rangle-\left\langle d_{1}^{\dagger} d_{2}\right\rangle-\left\langle d_{2}^{\dagger} d_{1}\right\rangle+\left\langle d_{2}^{\dagger} d_{2}\right\rangle\right],
\end{aligned}
$$

que são obtidas a partir da transformação (3.4). As curvas para $n_{\uparrow}$ e $n_{\downarrow}$ estão apresentadas a seguir em diferentes regimes. 

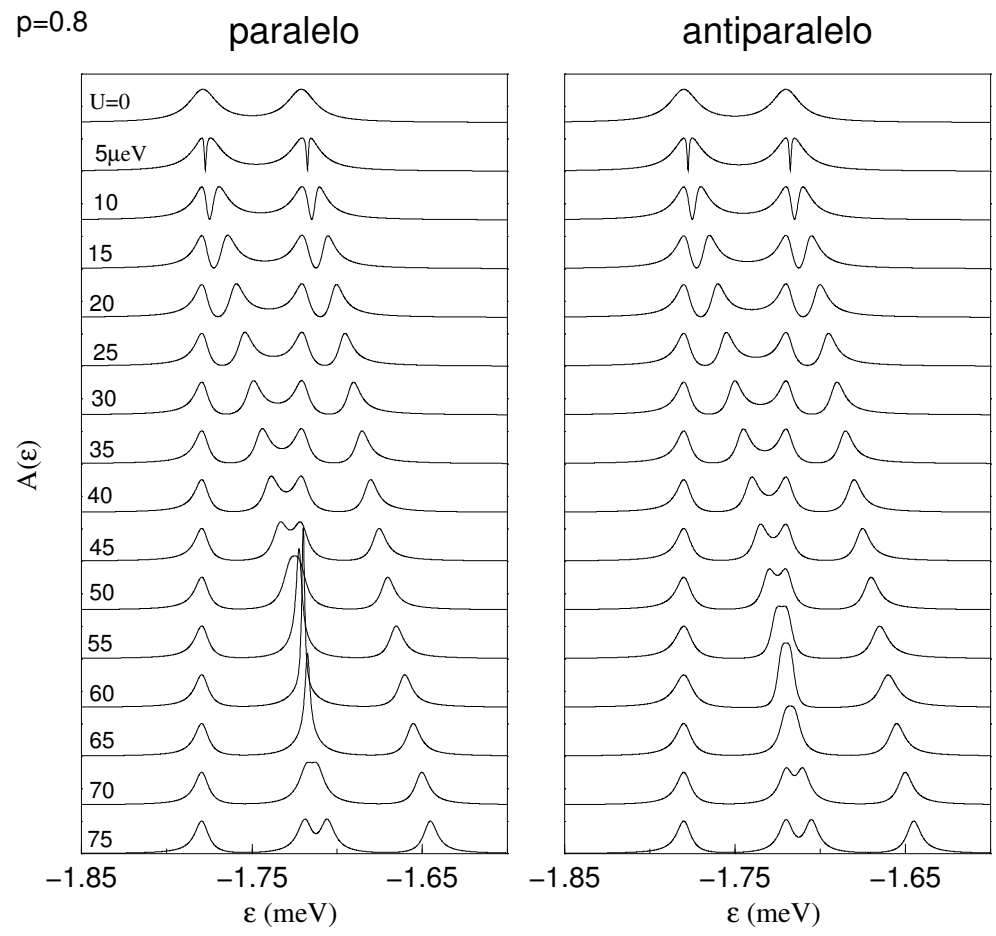

Figura 4.5: Função espectral nas configurações $\mathrm{P}$ e AP, $R=30 \mu \mathrm{eV}$ e diferentes valores de $U$. Para $U=0$ temos os picos $\epsilon_{1}$ e $\epsilon_{2}$. Para $U \neq 0$ surgem os picos $\epsilon_{1}+U$ e $\epsilon_{2}+U$ que se deslocam para a direita com o aumento de $U$. Quando $U \approx \epsilon_{2}-\epsilon_{1}$ os picos $\epsilon_{1}+U$ e $\epsilon_{2}$ se sobrepõem, dando origem a um pico estreito na configuração paralela. Esse estreitamento deve-se a um mecanismo de interferência que tende a desacoplar as componentes de spin $\uparrow$ e $\downarrow$, mesmo na presença de espalhamento de spin. Esse efeito será discutido posteriormente. Observe que na configuração AP a sobreposição de $\epsilon_{1}+U$ com $\epsilon_{2}$ não leva ao aparecimento de um pico estreito. Para $U \gtrsim 75 \mathrm{meV}$ a função espectral nas duas configurações se assemelham.

\subsubsection{Ocupação eletrônica no caso simétrico $p_{L}=p_{R}$}

Comecemos avaliando as ocupações $n_{\uparrow}$ e $n_{\downarrow}$ para o caso em que os terminais emissor e coletor são do mesmo material. Isso aparece em nosso formalismo através dos parâmetros de polarização $p_{L}$ e $p_{R}$. Quando $p_{L}=p_{R}$ temos emissor e coletor de mesmo material, e quando $p_{L} \neq p_{R}$ temos materiais diferentes. Na subseção seguinte analisamos o caso $p_{L} \neq p_{R}$.

Na figura (4.6) mostramos $n_{\uparrow}$ e $n_{\downarrow}$ contra tensão externa $V$ para $U=0, U=1$ meV, e configurações $\mathrm{P}$ e AP. Os triângulos $\triangle$ e $\nabla$ denotam spins $\uparrow$ e $\downarrow$, respectivamente. Enquanto na configuração paralela [painel (a)] as ocupações $n_{\uparrow}$ e $n_{\downarrow}$ coincidem, temos $n_{\uparrow} \neq n_{\downarrow}$ no alinhamento anti-paralelo [painel (b)]. Esse desbalanço das populações de spin obtido no caso AP é conhecido, no contexto de pontos quânticos metálicos, como acúmulo de spin [37],[82], e deve-se aos valores de $\Gamma_{\sigma}^{L}$ e $\Gamma_{\sigma}^{R}$.

Por exemplo, no caso paralelo temos $\Gamma_{\sigma}^{L}=\Gamma_{\sigma}^{R}$, assim, a taxa com que os elétrons 
de spin $\sigma$ entram no ponto quântico (emissor $\rightarrow \mathrm{PQ}$ ) é igual a taxa com que eles saem (PQ $\rightarrow$ coletor). Desse modo, o acúmulo de spins é o mesmo para as duas componentes $\uparrow$ e $\downarrow$. Já no caso AP, se a tensão é positiva ${ }^{1}$, a taxa de entrada de spins $\uparrow\left(\Gamma_{\uparrow}^{L}=14 \mu \mathrm{eV}\right)$, é maior do que a taxa de saída $\Gamma_{\uparrow}^{R}=6 \mu \mathrm{eV}$, enquanto para spins $\downarrow$ ocorre o oposto $\left(\Gamma_{\downarrow}^{L}=6 \mu \mathrm{eV}<\Gamma_{\downarrow}^{R}=14 \mu \mathrm{eV}\right)$. Como os spins $\uparrow$ entram no ponto quântico mais facilmente do que os spins $\downarrow\left(\Gamma_{\uparrow}^{L}>\Gamma_{\downarrow}^{L}\right)$ e saem com maior dificuldade $\left(\Gamma_{\uparrow}^{R}<\Gamma_{\downarrow}^{R}\right)$, o acúmulo é preferencialmente de spin $\uparrow$, resultando em $n_{\uparrow}>n_{\downarrow}$ [Fig.4.6(b)]. Para tensões negativas o terminal da direita funciona como emissor e o da esquerda como coletor de elétrons, o que leva a uma inversão no fluxo de portadores, e a análise em termos de taxas aplica-se da mesma forma trocando-se as taxas de entrada pelas de saída e vice-versa. Com isso obtemos $n_{\uparrow}<n_{\downarrow}$ [veja as curvas para $V<0$ na Fig.4.6(b)]. Vale ressaltar que encontramos um acúmulo de spin também na configuração paralela quando $p_{L} \neq p_{R}$, conforme discutiremos na seção seguinte.

Nas figuras 4.6(c) e (d) mostramos curvas da magnetização $m=n_{\uparrow}-n_{\downarrow}$ como função da tensão, para os alinhamentos $\mathrm{P}$ e AP, respectivamente. No caso paralelo [painel (c)] a magnetização é aproximadamente nula, visto que $n_{\uparrow} \approx n_{\downarrow}$. A pequena variação observada deve-se a diferença das larguras de linha dos níveis para spin $\uparrow$ e $\downarrow$. No alinhamento anti-paralelo vemos que $m$ troca de sinal quando passamos de tensões positivas para tensões negativas. Isso se deve a inversão das populações de spin de $V>0$ para $V<0$. As curvas com interação de Coulomb (linhas vermelhas) estão discutidas no próximo parágrafo.

\footnotetext{
${ }^{1}$ Para tensão positiva $(V>0)$ o terminal da esquerda $(\mathrm{L})$ funcional como o emissor de elétrons, e o terminal da direita $(\mathrm{R})$ como coletor. Para tensões negativas temos o contrário, o terminal da esquerda é o coletor, e o terminal da direita o emissor.
} 
paralelo
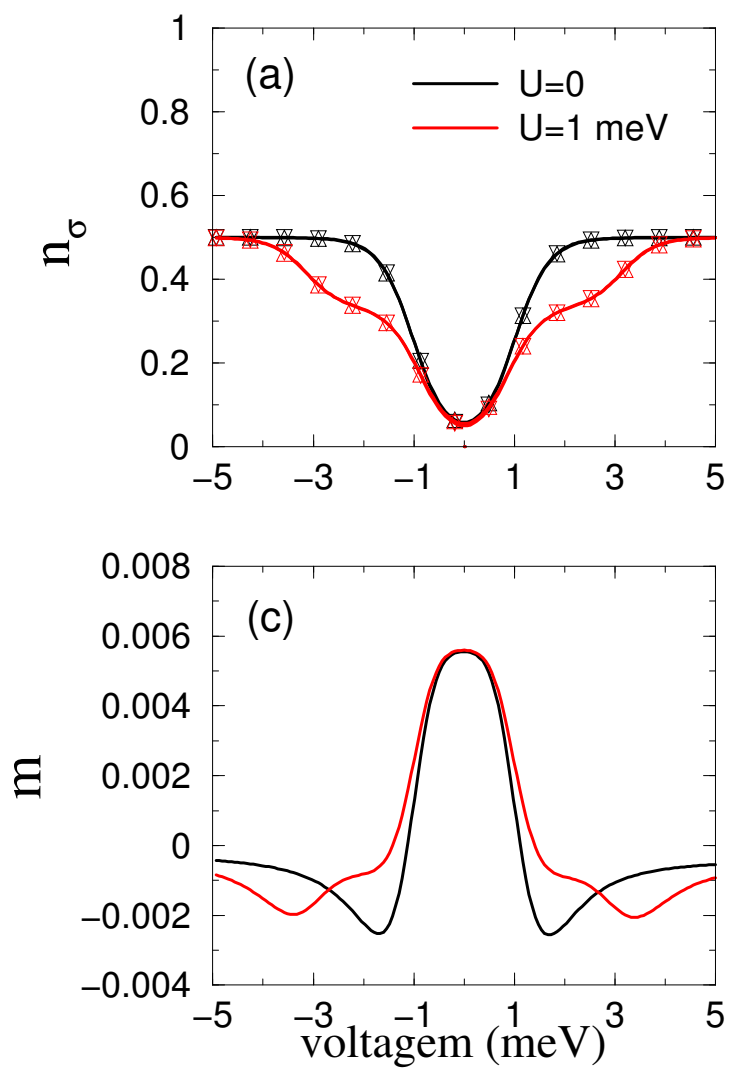

antiparalelo
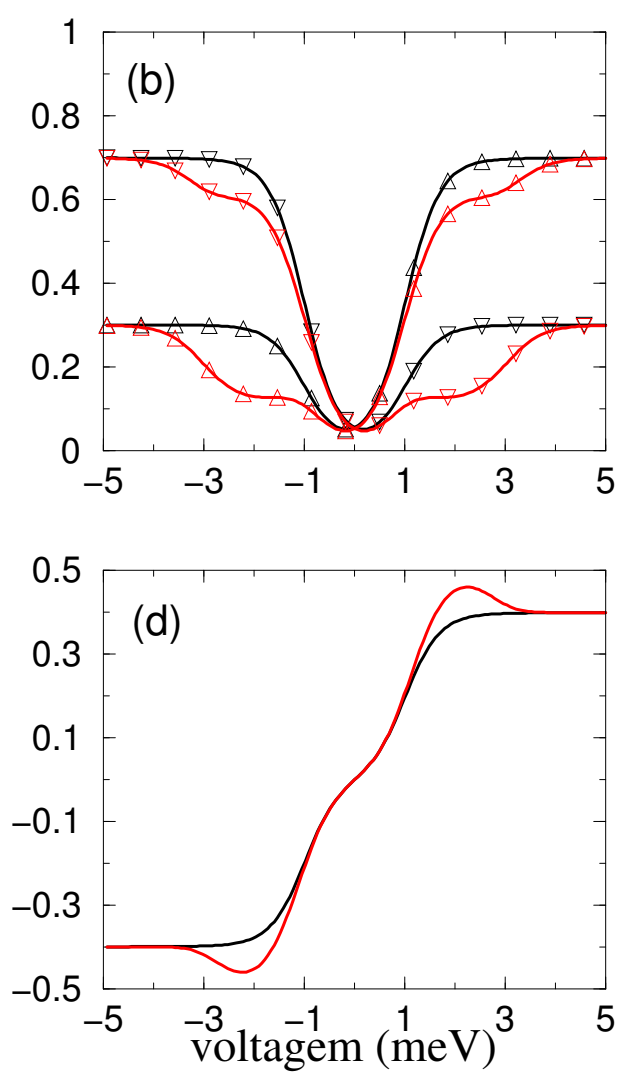

Figura 4.6: Ocupações $n_{\uparrow}(\triangle)$ e $n_{\downarrow}(\nabla)$ e magnetização $m=n_{\uparrow}-n_{\downarrow}$ contra tensão $\mathrm{eV}$ para $U=0$ (curvas pretas), $U=1 \mathrm{meV}$ (curvas vermelhas), e configurações paralela e anti-paralela. Enquanto no alinhamento paralelo temos $n_{\uparrow} \approx n_{\downarrow}$, no alinhamento antiparalelo obtemos $n_{\uparrow}>n_{\downarrow}$ para $V>0$ e $n_{\uparrow}<n_{\downarrow}$ para $V<0$. Esse desbalanço das populações de spin deve-se as diferentes taxas de tunelamento para os elétrons entrarem e saírem do ponto quântico. No painel (c) vemos que $m$ é aproximadamente zero no alinhamento $\mathrm{P}$, e apresenta uma pequena modulação em torno da voltagem nula, que se deve à diferença das larguras dos níveis para spin $\uparrow$ e $\downarrow$. Em (d) temos que $m$ é positivo para $V>0$ e negativo para $V<0$. Além disso, essa magnetização se intensifica na faixa de tensões em que as populações $n_{\uparrow}$ e $n_{\downarrow}$ são suprimidas pelo bloqueio de Coulomb $(1 \lesssim e V \lesssim 3 \mathrm{meV})$. Os parâmetros utilizados foram $p_{L}=p_{R}=0.4, k_{B} T=167 \mu \mathrm{eV}$, $\Gamma_{0}=10 \mu \mathrm{eV}, \epsilon_{0}=0.5 \mathrm{meV}$. 
Comparando os casos $U=0$ e $U=1 \mathrm{meV}$ na Fig.(4.6), notamos que a interação de Coulomb reduz as ocupações na faixa de tensões que varia de $1 \mathrm{meV}$ a $3 \mathrm{meV}$. Isso deve-se ao bloqueio de Coulomb que ocorre quando um elétron no ponto quântico impede a entrada de um segundo elétron via repulsão coulombiana. A Fig.(4.7) ilustra a condição em que ocorre esse bloqueio. Para $\mathrm{eV}=0$ [Fig.4.7(a)] temos que o nível $\epsilon_{d}$ encontra-se acima de $\mu_{L}$ e $\mu_{R}$ e portanto deve estar vazio, visto que os elétrons dos reservatórios não tem energia suficiente para ocupar esse nível. Quando $e V / 2=\epsilon_{d}$ [Fig.4.7(b)] o nível $\epsilon_{d}$ entra em ressonância com os estados do emissor, permitindo o tunelamento de um elétron para dentro do ponto quântico. Esse elétron permanecerá no ponto quântico por um certo tempo até sair para o terminal coletor. Nesse tempo de permanência no ponto quântico, um segundo elétron fica proibido de entrar a menos que tenha energia suficiente para vencer a repulsão coulombiana $U$. Essa situação está ilustrada na Fig.4.7(b) onde indicamos que não há transição de um segundo elétron do terminal para o ponto quântico, visto que o canal $\epsilon_{d}+U$ encontra-se muito acima de $\mu_{L}$.

Quando $e V / 2=\epsilon_{d}+U$, os elétrons do emissor adquirem energia suficiente para superar o bloqueio de Coulomb, podendo então entrar no ponto quântico. Note que o canal $\epsilon_{d}+U$ entra em ressonância com os estados do emissor a partir de $e V / 2=\epsilon_{d}+U$. Essa situação está ilustrada na Fig.4.7(c). Nesse caso dizemos que a tensão externa forneceu energia suficiente para os elétrons do emissor vencerem o bloqueio de Coulomb. Na Fig.4.6 isso ocorre para $|e V| \gtrsim 3 \mathrm{meV}$, quando as populações $n_{\uparrow}$ e $n_{\downarrow}$ (curvas vermelhas) tornam a crescer.

Retomando a figura (4.6), observamos ainda que, no caso AP [Fig.4.6(b)], a supressão da população minoritária $\left(n_{\downarrow}\right.$ para $V>0$ e $n_{\uparrow}$ para $\left.V<0\right)$ é maior do que a supressão da população majoritária $\left(n_{\uparrow}\right.$ para $V>0$ e $n_{\downarrow}$ para $\left.V<0\right)$. Isso ocorre porque quanto maior for a ocupação de um determinado spin, maior será o bloqueio de Coulomb sobre o spin oposto. Tomemos como exemplo a faixa de tensões positivas. Como $n_{\uparrow}>n_{\downarrow}$, quando há interação de Coulomb $(U=1$ meV) a ocupação $n_{\downarrow}$ é mais fortemente suprimida do que a ocupação $n_{\uparrow}$ dentro do regime de bloqueio de Coulomb. A esse efeito chamamos de bloqueio de Coulomb dependente de spin ${ }^{2}$. No caso $\mathrm{P}$ [Fig.4.6(a)] observamos apenas o bloqueio de Coulomb usual (não dependente de spin), visto que a magnetização no ponto quântico é essencialmente nula. Esse bloqueio de Coulomb dependente de spin leva a efeitos interessantes sobre a polarização da corrente, conforme veremos na Sec.4.4.

Notamos ainda que a magnetização intensifica-se no regime de bloqueio de Coulomb dependente de spin [compare curvas preta e vermelha no painel (d)]. Isso ocorre porque, como a supressão sobre uma dada população de spin é maior do que sobre a outra, a diferença $n_{\uparrow}-n_{\downarrow}$ tende a aumentar (em módulo).

\footnotetext{
${ }^{2}$ Um tipo de bloqueio de Coulomb dependente de spin na junção metálica ferromagnetometal-normal-ferromagneto (FM-NM-FM) foi analisada teoricamente em [82].
} 


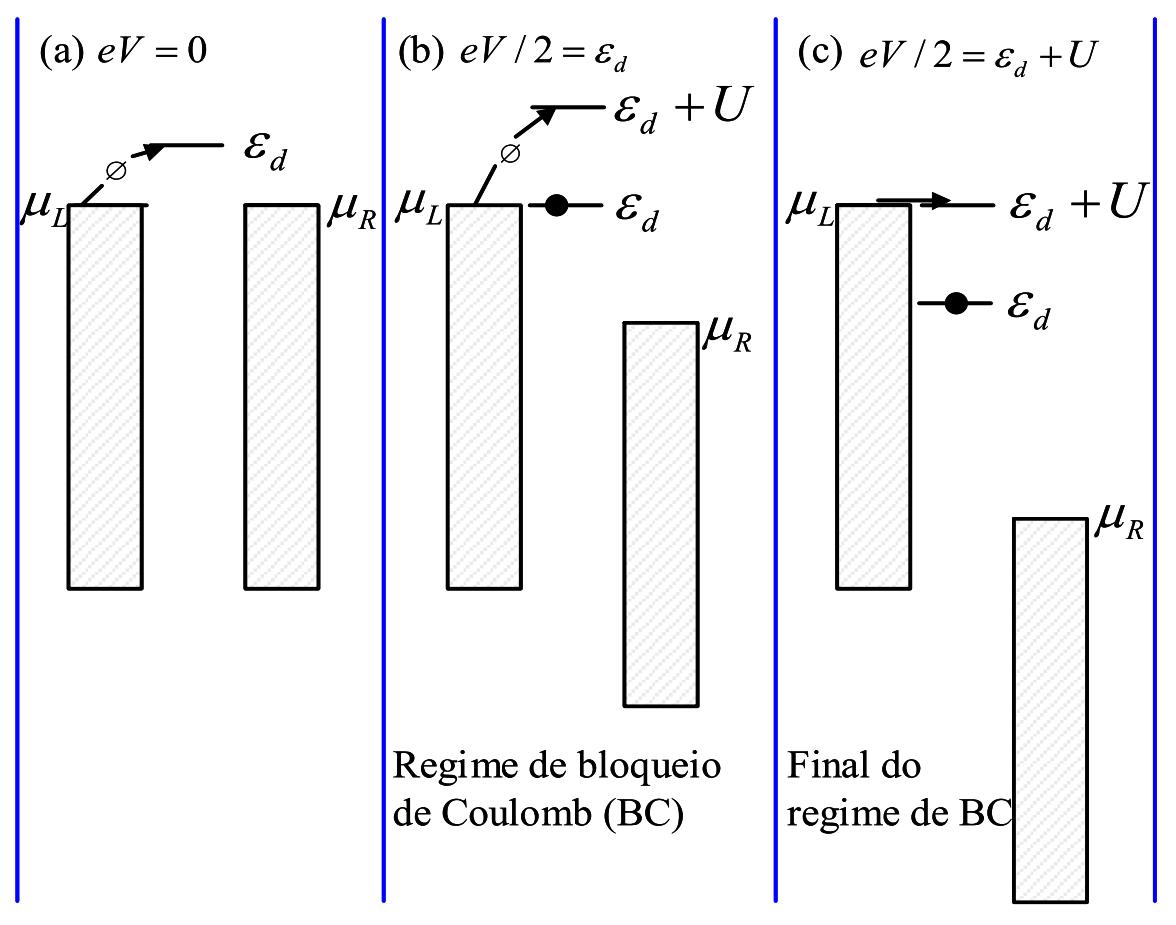

Figura 4.7: Diagrama de níveis de energia para o sistema de dois terminais acoplados a um ponto quântico. Consideramos que os terminais emissor e coletor tem uma banda constante que está populada até $\mu_{L}$ para o terminal da esquerda e $\mu_{R}$ para o terminal da direita. O ponto quântico apresenta um nível discreto $\epsilon_{d}$. Para $e V=0$ [painel (a)] temos que $\epsilon_{d}$ está acima de $\mu_{L}$ e de $\mu_{R}$, e portanto não está populado. Quando $e V / 2=\epsilon_{d}$ [painel (b)] o nível $\epsilon_{d}$ entra em ressonância com os estados do terminal esquerdo, podendo assim ser ocupado por um elétron. Enquanto esse estado estiver ocupado, um segundo elétron só poderá entrar no ponto quântico se tiver energia suficiente para vencer a repulsão coulombiana. No painel (b), por exemplo, indicamos que um segundo elétron não pode "entrar" no ponto quântico, visto que sua energia $\left(\mu_{L}\right)$ é menor do que a energia de excitação $\epsilon_{d}+U$. Quando a tensão vale $e V / 2=\epsilon_{d}+U$ os elétrons do emissor adquirem energia suficiente para entrar no ponto quântico, visto que $\epsilon_{d}+U$ entra em ressonância com os estados do emissor [painel (c)]. 


\subsubsection{Ocupação eletrônica no caso assimétrico $p_{L} \neq p_{R}$}

Analisamos na subseção anterior as populações $n_{\uparrow}$ e $n_{\downarrow}$ no caso $p_{L}=p_{R}$, isto é, quando os terminais emissor e coletor são do mesmo material. Quando o terminal emissor é de um material e o terminal coletor de outro $\left(p_{L} \neq p_{R}\right)$, por exemplo um de $\mathrm{Ni}$ e outro de $\mathrm{Co}$, surge um efeito adicional, conforme observamos na próxima figura.

Na Fig.(4.8) apresentamos curvas semelhantes as da Fig.(4.6), exceto pelos valores de $p_{L}$ e de $p_{R}$ utilizados, $p_{L}=0.23$ e $p_{R}=0.35$ [83]. No caso antiparalelo [Fig.4.8(b)] não observamos nenhum contraste com relação ao caso $p_{L}=$ $p_{R}$ [Fig.4.6(b)]. Já no alinhamento paralelo [Fig.4.8(a)] temos $n_{\uparrow}<n_{\downarrow}$ para $V>0$ e $n_{\uparrow}>n_{\downarrow}$ para $V<0$, desigualdades que não ocorriam no caso $p_{L}=p_{R}$ [Fig.4.6(a)]. Essas desigualdades dão origem a uma magnetização não nula do ponto que pode levar ao bloqueio de Coulomb dependente de spin no caso paralelo.

O desbalanço das ocupações $n_{\uparrow}$ e $n_{\downarrow}$, observado no caso paralelo, pode ser entendido através das taxas $\Gamma_{\sigma}^{L}$ e $\Gamma_{\sigma}^{R}$. Para $p_{L}=0.23$ e $p_{R}=0.35$ temos os seguintes valores para a configuração paralela:

$$
\begin{aligned}
& \Gamma_{\uparrow}^{L}=12.3 \mu \mathrm{eV}, \Gamma_{\uparrow}^{R}=13.5 \mu \mathrm{eV} \\
& \Gamma_{\downarrow}^{L}=7.7 \mu \mathrm{eV}, \Gamma_{\downarrow}^{R}=6.5 \mu \mathrm{eV},
\end{aligned}
$$

onde adotamos $\Gamma_{0}^{L}=\Gamma_{0}^{R}=\Gamma_{0}=10 \mu \mathrm{eV}$ [81]. Com esses parâmetros vemos que, para tensões positivas $V>0$, os elétrons de spin $\uparrow$ deixam o ponto quântico mais rápido do que entram $\left(\Gamma_{\uparrow}^{L}<\Gamma_{\uparrow}^{R}\right)$, e o oposto se dá para spins $\downarrow\left(\Gamma_{\downarrow}^{L}>\Gamma_{\downarrow}^{R}\right)$. Logo, há um acúmulo preferencial de spins $\downarrow$ no ponto quântico, o que explica o desbalanço $n_{\uparrow}<n_{\downarrow}$ [Fig.4.8(a)]. Para $V<0$ trocamos as taxas de entrada pela de saída e vice-versa, resultando numa inversão das populações $\uparrow$ e $\downarrow$. No alinhamento AP invertemos as taxas da direita $\Gamma_{\sigma}^{R}\left(\Gamma_{\uparrow}^{R} \rightleftarrows \Gamma_{\downarrow}^{R}\right)$, o que resulta em $n_{\uparrow}>n_{\downarrow}$ para $V>0$ e $n_{\uparrow}<n_{\downarrow}$ para $V<0$, conforme observamos na Fig.4.8(b).

Nos painéis (c) e (d) da Fig.(4.8) mostramos os gráficos das magnetizações $m=n_{\uparrow}-n_{\downarrow}$. Como para $p_{L} \neq p_{R}$ temos $n_{\uparrow} \neq n_{\downarrow}$ (no alinhamento P), a magnetização aqui é bem maior do que a obtida no caso $p_{L}=p_{R}[$ Fig.4.6(c)]. Além disso, observamos uma intensificação de $m$ no regime de bloqueio de Coulomb [compare curvas preta e vermelha da Fig.4.8(c)], semelhantemente ao caso antiparalelo [Fig.4.8(d)]. Comparando os painéis (c) e (d) vemos que é possível flipar a magnetização do ponto quântico variando o alinhamento magnético dos eletrodos. Por exemplo, para $\mathrm{eV}=5 \mathrm{meV}$ temos uma magnetização negativa no caso paralelo e positiva no alinhamento anti-paralelo. 

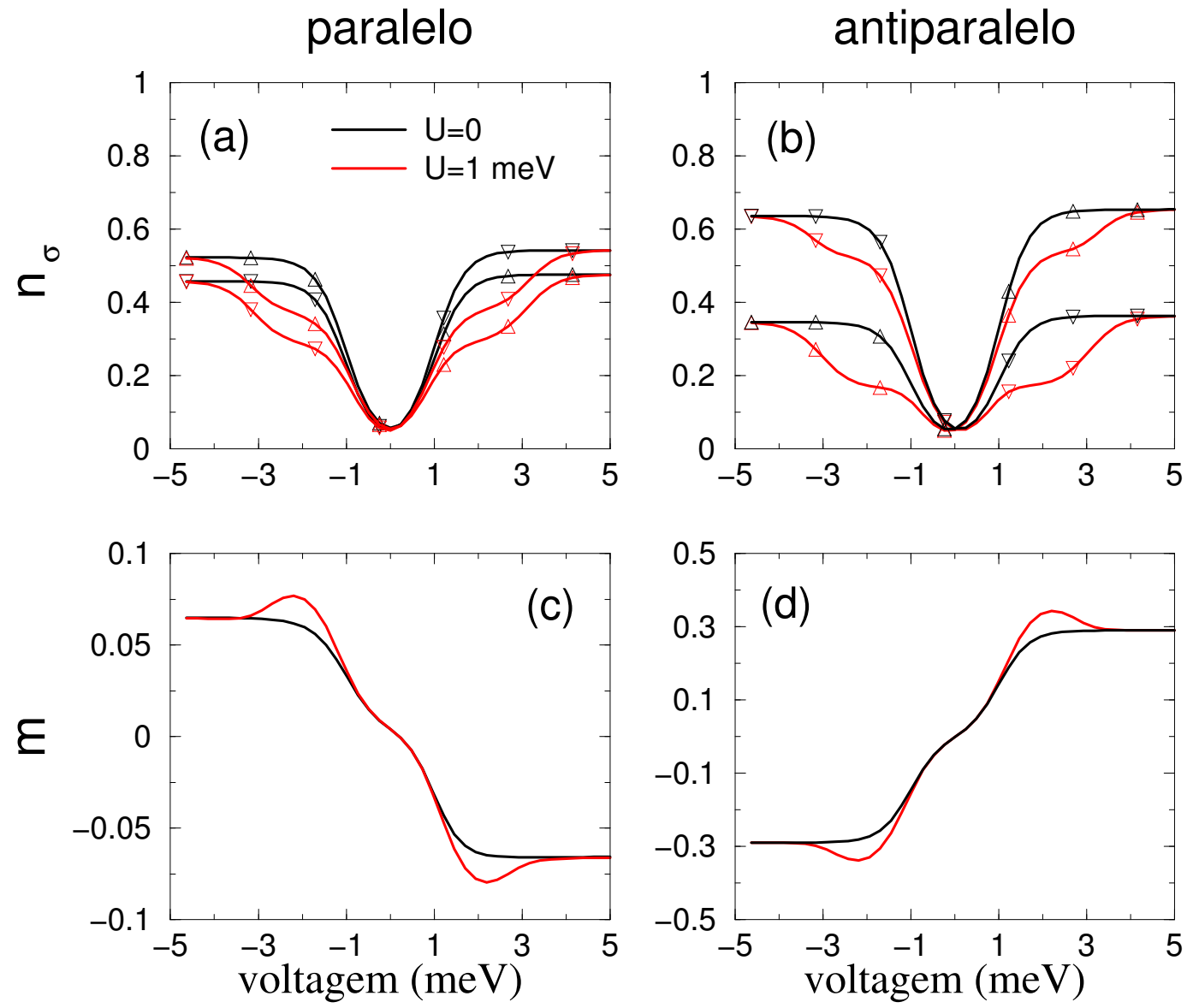

Figura 4.8: Curvas análogas as da Fig.(4.6) exceto pelos valores de $p_{L}$ e de $p_{R}$ utilizados, $p_{L}=0.23$ e $p_{R}=0.35$. A principal diferença dessas curvas com relação as ilustradas no caso simétricos $p_{L}=p_{R}$ [Fig.(4.6)] está no fato de termos uma diferença apreciável entre $n_{\uparrow}$ e $n_{\downarrow}$ no alinhamento paralelo. Note que $n_{\uparrow}<n_{\downarrow}$ para $\mathrm{eV}>0$ e $n_{\uparrow}>n_{\downarrow}$ para $e V<0$ nesse alinhamento. Com relação às magnetizações, vemos que $m$ muda de sinal ao passarmos de tensões positivas para tensões negativas. Ressaltamos ainda que, para uma dada voltagem (digamos $e V=5 \mathrm{meV}$ ), a magnetização do ponto quântico muda de sinal quando o alinhamento magnético varia de $\mathrm{P}$ para AP, o que mostra ser possível controlar a magnetização do ponto quântico através da configuração magnética do sistema. Os parâmetros adotados aqui foram: $p_{L}=0.23, p_{R}=0.35, k_{B} T=167 \mu \mathrm{eV}$, $\Gamma_{0}=10 \mu \mathrm{eV}, \epsilon_{0}=0.5 \mathrm{meV}$. 


\subsubsection{Ocupações como função de $\mathbf{U}[\mathrm{eV}=4 \mathrm{meV}, R=0]$}

Na figura (4.9) mostramos $n_{\uparrow}$ e $n_{\downarrow}$ como função de $U$ para diferentes parâmetros de acoplamento $\Gamma_{0}^{R}$, e uma tensão constante $(e V=4 \mathrm{meV})$. O parâmetro $\Gamma_{0}^{L}$ foi fixado em $10 \mu \mathrm{eV}$. Note que para tensões positivas $\Gamma_{0}^{L}$ corresponde a taxa de entrada de elétrons no ponto quântico e $\Gamma_{0}^{R}$ a taxa de saída. Portanto, ao diminuirmos $\Gamma_{0}^{R}$ estamos "dificultando" a saída de elétrons do ponto quântico, e portanto esses tendem a se acumular mais no ponto quântico. A tensão $e V=4 \mathrm{meV}$ permite que os canais $\epsilon_{d}$ e $\epsilon_{d}+U$ estejam em ressonância com os estados do emissor para $U<1.5 \mathrm{meV}$, isto é, estamos acima do regime de bloqueio de Coulomb.

Na configuração paralela [Fig.4.9(a)] as ocupações $n_{\uparrow}$ e $n_{\downarrow}\left(n_{\uparrow} \approx n_{\downarrow}\right)$ são inicialmente constantes $(U<1 \mathrm{meV})$, começam a decrescer a partir de $U \approx 1 \mathrm{meV}$, e atingem valores novamente constantes para $U>2 \mathrm{meV}$. Essa supressão das populações de spin deve-se ao fato do nível $\epsilon_{d}+U$ sair de ressonância em torno de $U \approx 1.5 \mathrm{meV}$. Como $\epsilon_{d}=\epsilon_{0}-e V / 2=-1.5 \mathrm{meV}$, o nível $\epsilon_{d}+U$ esta originalmente $(U \rightarrow 0)$ bem abaixo de $\mu_{L}=0$, mas, à medida que $U$ aumenta, esse nível cresce em energia e eventualmente fica acima de $\mu_{L}$ saindo de ressonância com os estados do emissor. Quando isso ocorre dizemos que o sistema entra no regime de bloqueio de Coulomb, e conseqüentemente as populações decrescem. Na Fig.(4.9) o bloqueio de Coulomb ocorre para $U=1.5 \mathrm{meV}$, porém, devido a largura dos níveis e efeitos térmicos, a supressão das ocupações se dá um pouco antes $(U \approx 1$ $\mathrm{meV})$.

Note que para $U<1 \mathrm{meV}$, as ocupações $n_{\uparrow}$ e $n_{\downarrow}$ tendem a 1 à medida que $\Gamma_{0}^{R} \rightarrow 0$. Isso ocorre porque a diminuição de $\Gamma_{0}^{R}$ leva a um maior acúmulo de elétrons no ponto quântico (os elétrons se empilham mais), favorecendo assim dupla ocupação ${ }^{3}$. No regime de bloqueio de Coulomb $(U>2 \mathrm{meV})$, dupla ocupação é proibida, visto que um elétron bloqueia a entrada de um segundo elétron. Logo, $n_{\uparrow}$ e $n_{\downarrow}$ tendem a 0.5 quando $\Gamma_{0}^{R} \rightarrow 0$, o que corresponde a ocupação simples (um único elétron no ponto quântico). Note que a soma $n_{\uparrow}+n_{\downarrow}$ não ultrapassa 1 no regime de bloqueio de Coulomb, visto que o ponto quântico comporta no máximo um elétron nesse regime. Já para $U<1 \mathrm{meV}$ temos que $n_{\uparrow}+n_{\downarrow} \rightarrow 2$ quando $\Gamma_{0}^{R} \rightarrow 0$, o que equivale a dupla ocupação.

Na configuração antiparalela [Fig.4.9(b)] também se observa a supressão das ocupações para $U \gtrsim 1 \mathrm{meV}$, e os limites $\left(n_{\uparrow}+n_{\downarrow}\right) \rightarrow 2$ para $U<1.5 \mathrm{meV}$ e $\left(n_{\uparrow}+n_{\downarrow}\right) \rightarrow 1$ para $U>1.5 \mathrm{meV}$, porém a variação como função de $\Gamma_{0}^{R}$ difere do caso paralelo. Enquanto $n_{\downarrow}$ cresce monotonicamente à medida que $\Gamma_{0}^{R}$ decresce, $n_{\uparrow}$ apresenta um comportamento não monotônico. Para $U>1.5 \mathrm{meV}$ temos que $n_{\uparrow}$ inicialmente cresce (compare linhas preta, vermelha e verde) e em seguida decresce (veja linha azul) à medida que $\Gamma_{0}^{R}$ diminui. Essa variação deve-se a uma competição

\footnotetext{
${ }^{3}$ Isso é verdade somente para $V>0$, onde $\Gamma_{0}^{R}$ corresponde a taxa de saída de elétrons do ponto quântico.
} 
entre dois efeitos: (1) por um lado $n_{\uparrow}$ aumenta devido a diminuição de $\Gamma_{0}^{R}$, que tende a "empilhar" mais elétrons, e por outro (2) $n_{\uparrow}$ tende a ser mais bloqueado coulombicamente pela população $n_{\downarrow}$, que cresce a medida que $\Gamma_{0}^{R}$ diminui. Dessa competição resulta a variação não monotônica de $n_{\uparrow}$ observada para $U>1.5 \mathrm{meV}$ no alinhamento AP.
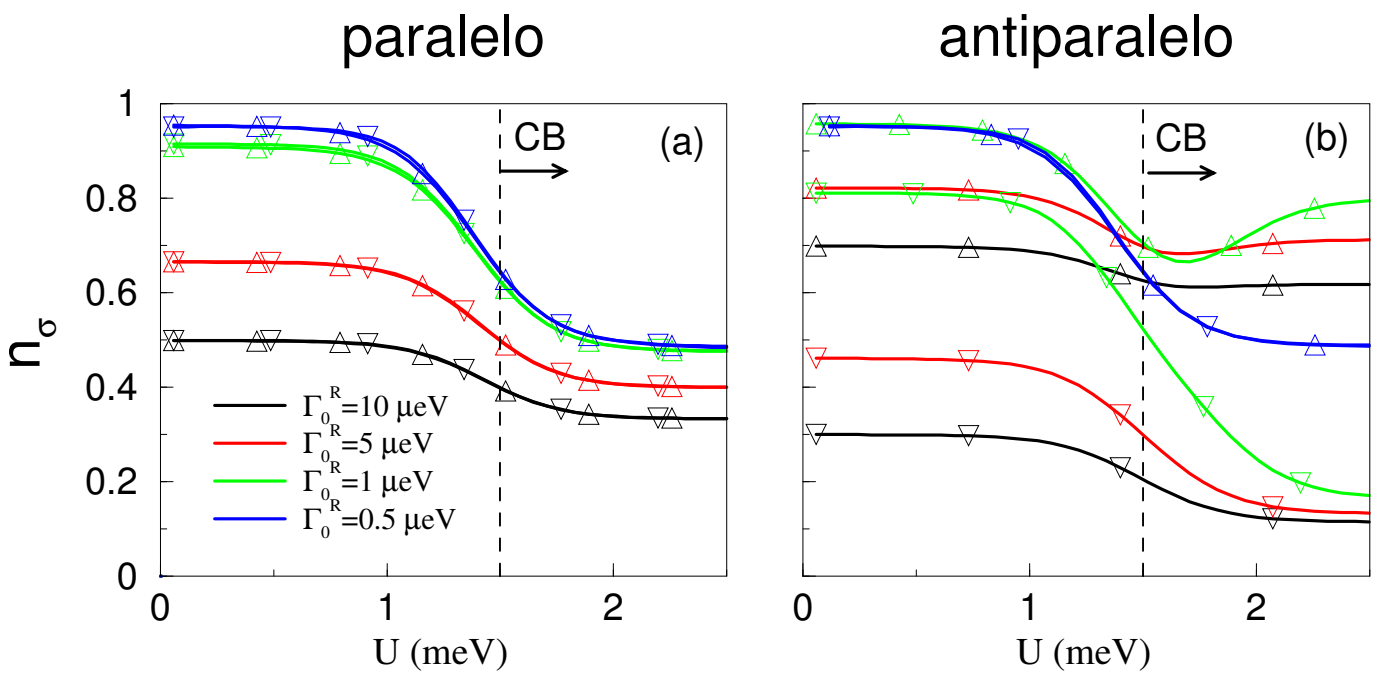

Figura 4.9: Ocupações $n_{\uparrow}(\triangle)$ e $n_{\downarrow}(\nabla)$ versus $U$ para diferentes valores de $\Gamma_{0}^{R}$. Observamos a supressão de $n_{\uparrow}$ e $n_{\downarrow}$ para $U \gtrsim 1 \mathrm{meV}$ nas duas configurações magnéticas $\mathrm{P}$ e AP. Essa supressão deve-se ao bloqueio de Coulomb. A tensão utilizada foi $\mathrm{eV}=4$ meV. Com isso temos $\epsilon_{d}=\epsilon_{0}-e V / 2=-1.5 \mathrm{meV}$, que é um valor menor do que $\mu_{L}$ $\left(\mu_{L}=0\right)$. Para $U \geqslant 1.5 \mathrm{meV}$ o canal $\epsilon_{d}+U$ adquire energia superior a $\mu_{L}$ e com isso os elétrons do emissor deixam de tunelar para o ponto quântico, o que leva a redução das ocupações $n_{\uparrow}$ e $n_{\downarrow}$ observada nessa figura. Notamos que, para $\Gamma_{0}^{R} \rightarrow 0$, ambas as ocupações $n_{\uparrow}$ e $n_{\downarrow}$ tendem a 1 para $U \lesssim 1 \mathrm{meV}$. Isso significa que dupla-ocupação é favorecida a medida que $\Gamma_{0}^{R}$ diminui, isto é, $\left(n_{\uparrow}+n_{\downarrow}\right) \rightarrow 2$. No regime de bloqueio de Coulomb $\left(U \gtrsim 1.5 \mathrm{meV}\right.$ ) essas ocupações tendem a $0.5 \mathrm{com}$ a diminuição de $\Gamma_{0}^{R}$, o que corresponde a ocupação simples, $\left(n_{\uparrow}+n_{\downarrow}\right) \rightarrow 1$. Notemos que no caso AP [painel (b)] a ocupação $n_{\uparrow}$ inicialmente aumenta com a diminuição de $\Gamma_{0}^{R}$ (veja curvas preta, vermelha e verde), e em seguida diminui (curva azul) para $\Gamma_{0}^{R}=0.5 \mu \mathrm{eV}$. Esse comportamento não-monotônico deve-se a uma competição entre a taxa de tunelamento $\Gamma_{0}^{R}$ e o bloqueio de Coulomb. Outros parâmetros: $p_{L}=p_{R}=0.4, k_{B} T=0.17 \mathrm{meV}, \epsilon_{0}=0.5 \mathrm{meV}$. 


\subsubsection{Efeitos do espalhamento de spin sobre $n_{\uparrow}$ e $n_{\downarrow}$}

Por último, analisamos os efeitos do espalhamento de spin sobre as populações $n_{\uparrow}$ e $n_{\downarrow}$. A figura 4.10 mostra $n_{\uparrow}$ e $n_{\downarrow}$ contra tensão $V$ para diferentes parâmetros de espalhamento de spin: $R=0,6,30 \mu \mathrm{eV}$. Observamos que o espalhamento de spin mistura as populações $n_{\uparrow}$ e $n_{\downarrow}$, tendendo a equilibrá-las. Como na configuração $\mathrm{P}$ já temos naturalmente $n_{\uparrow}=n_{\downarrow}$, o espalhamento de spin não produz nenhum efeito nesse caso. Já no caso AP temos $n_{\uparrow}>n_{\downarrow}$, logo o espalhamento de spin tende a suprimir $n_{\uparrow}$ e aumentar $n_{\downarrow}$, de modo a igualar essas duas populações. Observamos ainda que as curvas para $n_{\uparrow}$ e $n_{\downarrow}$ no alinhamento AP tendem para o mesmo valor do caso P quando $R$ é grande o suficiente [compare a curva da Fig.4.10(a) com as curvas para $R=30 \mu \mathrm{eV}$ do painel (b)].
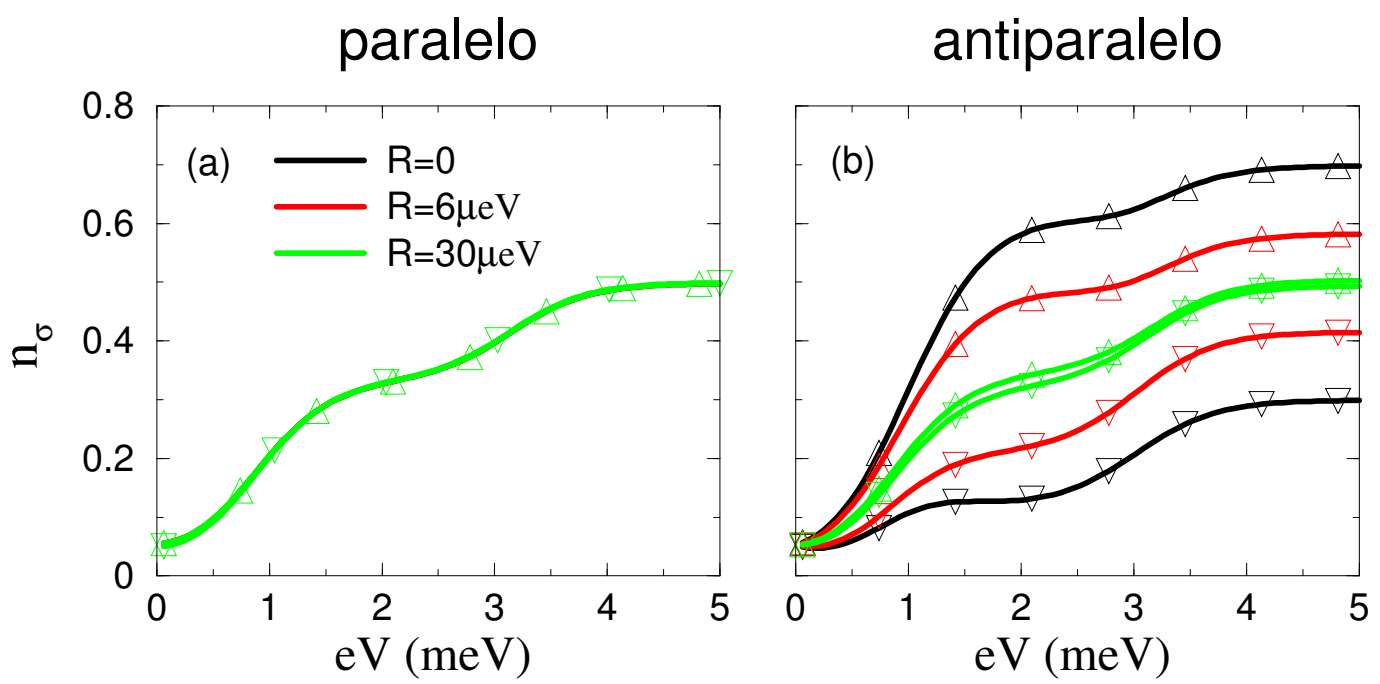

Figura 4.10: Ocupações $n_{\uparrow}(\triangle)$ e $n_{\downarrow}(\nabla)$ versus tensão nas configurações (a) paralela e (b) anti-paralela e diferentes parâmetros de espalhamento de spin. Aumentando o parâmetro de espalhamento de spin $R$ observamos que as ocupações $n_{\uparrow}$ e $n_{\downarrow}$ tendem a se igualar na configuração AP e são insensíveis a $R$ no alinhamento P. Portanto temos que o principal efeito do espalhamento de spin é equilibrar as populações de spin $n_{\uparrow} \mathrm{e}$ $n_{\downarrow}$. Como no alinhamento paralelo temos naturalmente $n_{\uparrow}=n_{\downarrow}$, o espalhamento de spin não surte nenhum efeito sobre essas populações. Os parâmetros utilizados aqui foram $U=1 \mathrm{meV}, p_{L}=p_{R}=0.4, \Gamma_{0}^{L}=\Gamma_{0}^{R}=10 \mu \mathrm{eV}, k_{B} T=0.17 \mathrm{meV}$.

\subsection{Corrente}

Nessa seção apresentamos gráficos para a corrente e a sua polarização como função da tensão externa $V$, e dos parâmetros $R$ e $U$. Alguns gráficos para $n_{\uparrow}$ e $n_{\downarrow}$ apresentados na seção anterior, foram reproduzidos aqui para facilitar nossa análise do comportamento da corrente. 


\subsubsection{Corrente e sua polarização no caso simétrico $\left[p_{L}=p_{R}\right]$}

Na figura (4.11) mostramos as ocupações $n_{\uparrow}(\triangle)$ e $n_{\downarrow}(\nabla)$, as correntes resolvidas em spin $I_{\uparrow}(\triangle)$ e $I_{\downarrow}(\nabla)$, e a polarização da corrente $\wp=\left(I_{\uparrow}-I_{\downarrow}\right) /\left(I_{\uparrow}+I_{\downarrow}\right)$ nas configurações paralela e anti-paralela, $U=0$ e $U=1 \mathrm{meV}$. As curvas para $n_{\uparrow}$ e $n_{\downarrow}$ [Fig.4.11(a)-(b)] já foram discutidas na seção anterior, por isso passamos diretamente para a análise da corrente. Nas figuras 4.11(c)-(d) temos $I_{\uparrow}$ e $I_{\downarrow}$ com e sem interação de Coulomb, linhas vermelha e preta, respectivamente, e nas configurações paralela [painel (c)] e antiparalela [painel (d)]. No caso paralelo temos $\left|I_{\uparrow}\right|>\left|I_{\downarrow}\right|$, tanto para $U=0$ quanto para $U=1 \mathrm{meV}$. A supressão das correntes $I_{\uparrow}$ e $I_{\downarrow}$ na faixa de tensões que varia de $1 \mathrm{meV}$ a $3 \mathrm{meV}$ deve-se ao bloqueio de Coulomb. Como nessa faixa de voltagem um segundo elétron fica energeticamente proibido de tunelar para o ponto quântico, a corrente tende a diminuir, conforme observamos nos gráficos.

No caso antiparalelo temos $I_{\uparrow}=I_{\downarrow}$ quando não há interação de Coulomb [Fig.4.11(d)], porém, na presença de interação de Coulomb as duas componentes $I_{\uparrow}$ e $I_{\downarrow}$ são suprimidas no regime de bloqueio de Coulomb, sendo $I_{\downarrow}$ mais intensamente suprimida do que $I_{\uparrow}$ para $V>0$ e o contrário para $V<0$. Essa diferença nas supressões de $I_{\uparrow}$ e $I_{\downarrow}$ deve-se justamente à diferença de ocupações $n_{\uparrow}$ e $n_{\downarrow}$ observadas na Fig.4.11(b). Como $n_{\uparrow}>n_{\downarrow}$ para $V>0$ temos que a corrente $I_{\downarrow}$ é mais fortemente suprimida [Fig.4.11(d)]. Do mesmo modo a corrente $I_{\uparrow}$ é mais suprimida do que $I_{\downarrow}$ para $V<0$ pois temos $n_{\uparrow}<n_{\downarrow}$ na faixa de tensões negativas.

A diferença nas supressões de $I_{\uparrow}$ e $I_{\downarrow}$ é claramente observada nas curvas de polarização apresentadas nos painéis (e)-(f). No caso paralelo a polarização da corrente com e sem interação de Coulomb é essencialmente a mesma visto que não há bloqueio de Coulomb dependente de spin $\left[n_{\uparrow} \approx n_{\downarrow}\right]$. O inset no painel (e) mostra uma ampliação da polarização na faixa em que ocorre uma pequena variação de sua intensidade. Essa variação deve-se a diferença das larguras dos níveis para spin $\uparrow \mathrm{e} \downarrow$. Vale ressaltar que esse mesmo efeito se manifesta na curva de magnetização do ponto quântico [Fig.4.6(c)]

Com relação à configuração AP, temos uma forte intensificação da polarização quando "ligamos" a interação de Coulomb [compare curvas preta e vermelha no painel (f)]. Em particular, para tensões negativas, além da polarização se intensificar (em módulo) ocorre uma inversão do seu sinal, isto é, para $V>0$ temos uma polarização positiva e para $V<0$ uma polarização negativa. Esse efeito se deve ao bloqueio de Coulomb dependente de spin que surge quando $n_{\uparrow} \neq n_{\downarrow}$. O sistema FM-PQ-FM então permite inverter a polarização da corrente trocando-se o sinal da tensão externa. Vale salientar que isso só ocorre na presença de interação de Coulomb. No caso $U=0$, a polarização é sempre nula no alinhamento anti-paralelo e aproximadamente constante na configuração paralela. Seguimos analisando o caso assimétrico $p_{L} \neq p_{R}$. 
paralelo
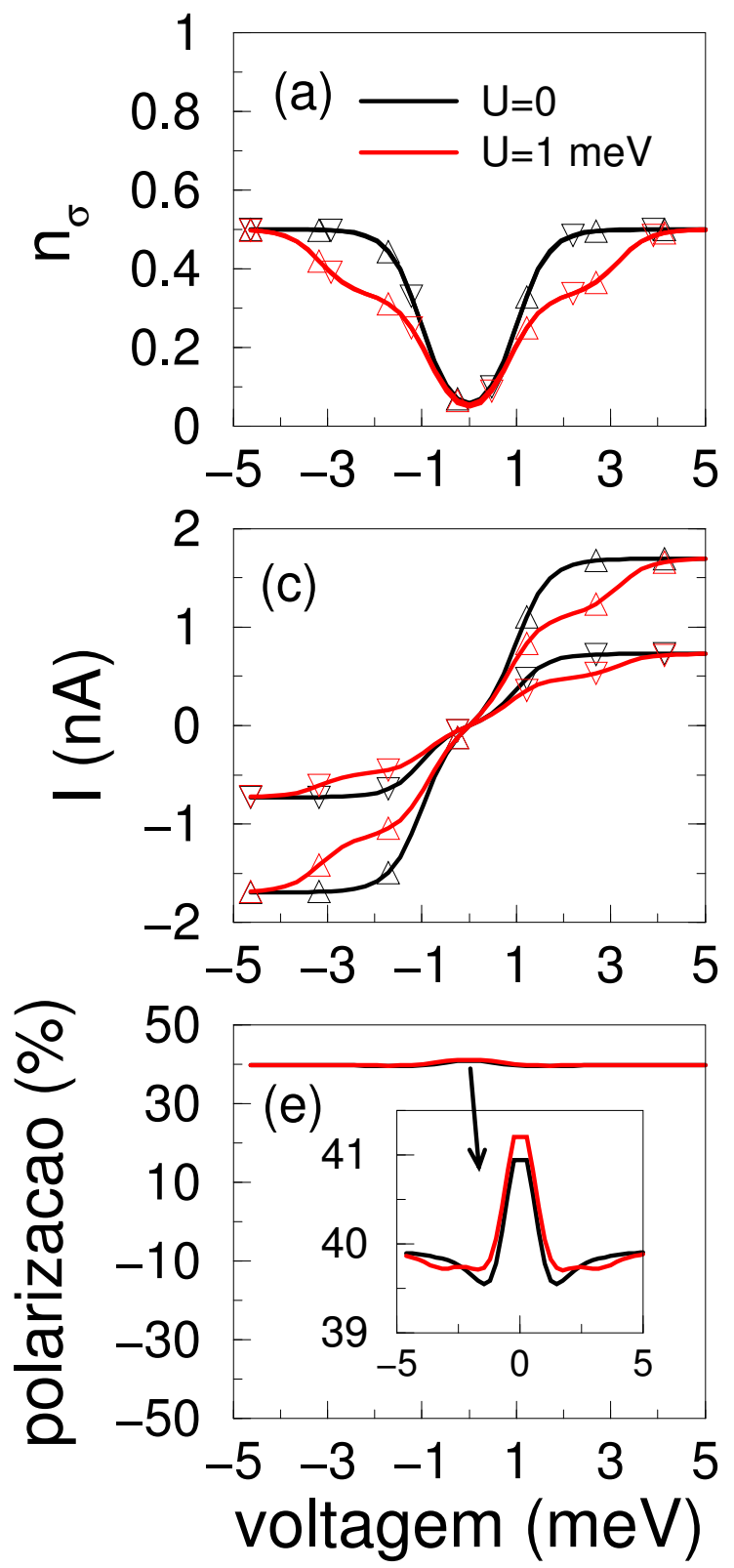

antiparalelo
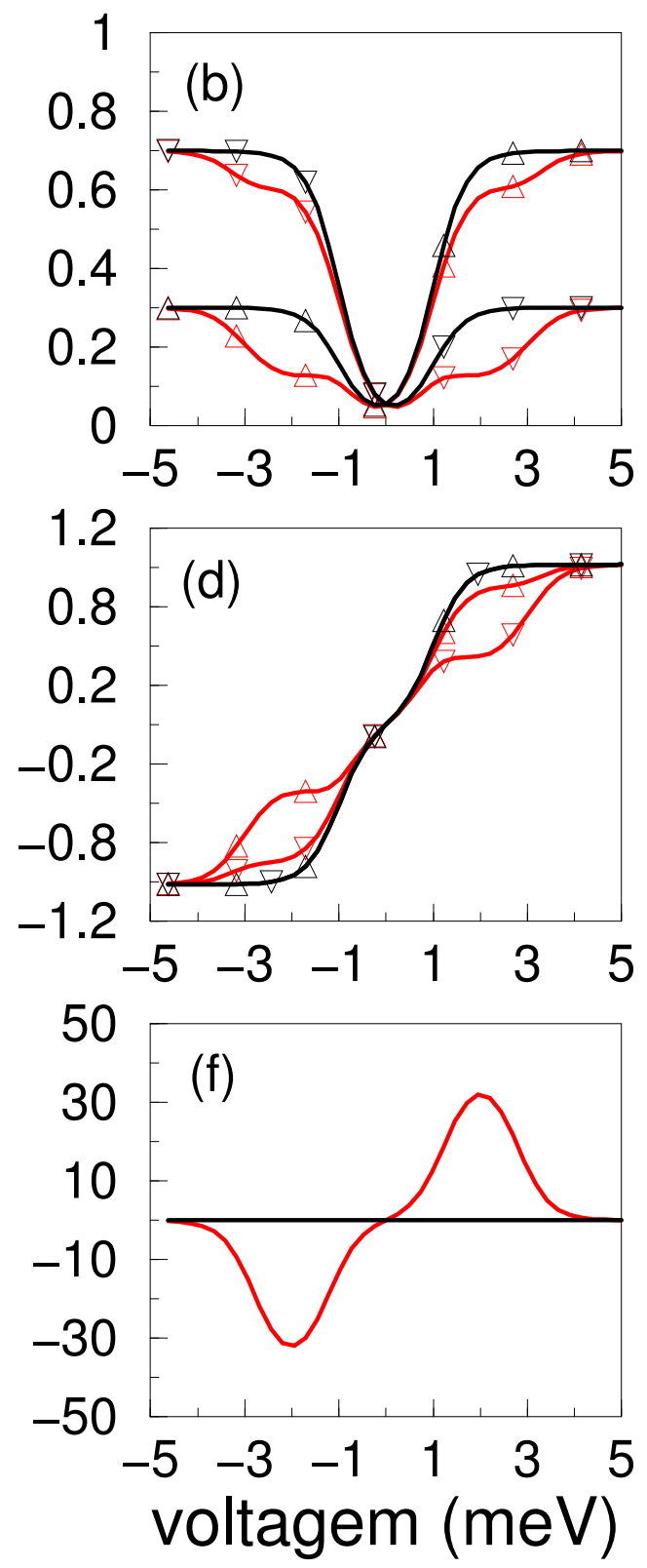

Figura 4.11: (a)-(b) Ocupações $n_{\uparrow}$ e $n_{\downarrow}$, (c)-(d) correntes $I_{\uparrow}$ e $I_{\downarrow}$, e (e)-(f) polarização nas configurações $\mathrm{P}$ e AP como função da voltagem para $p_{L}=p_{R}=0.4$. Os painéis (a)-(b) já foram apresentados anteriormente e estão aqui para facilitar nossa análise. No caso paralelo [painel (c)] temos $\left|I_{\uparrow}\right|>\left|I_{\downarrow}\right|$ tanto para $U=0$ quanto para $U=1 \mathrm{meV}$. Já na configuração AP [painel (d)] temos que $I_{\uparrow}=I_{\downarrow}$ para $U=0$ e $I_{\uparrow} \neq I_{\downarrow}$ para $U=1 \mathrm{meV}$ e regime de bloqueio de Coulomb. Essa diferença das correntes $I_{\uparrow}$ e $I_{\downarrow}$ é provocada pelo bloqueio de Coulomb dependente de spin. A polarização da corrente no caso paralelo é essencialmente constante, e no caso antiparalelo observamos uma grande variação de $\wp$ como função da tensão. Essa variação deve-se ao bloqueio de Coulomb dependente de spin que resulta numa polarização positiva para $V>0$ e negativa para $V<0$, isto é, temos uma polarização da corrente sintonizável pelo potencial. 


\subsubsection{Corrente e sua polarização no caso assimétrico $\left[p_{L} \neq\right.$}

$\left.p_{R}\right]$

Na figura (4.12) mostramos as curvas de (a)-(b) $n_{\sigma}$, (c)-(d) $I_{\sigma}$ e (e)-(f) polarização como função da tensão para os parâmetros $p_{L}=0.23$ e $p_{R}=0.35$. Os gráficos das ocupações já foram apresentados na seção anterior e estão aqui para guiar nossa discussão. Nos painéis (c)-(d) temos as curvas das correntes $I_{\uparrow}$ e $I_{\downarrow}$. No caso paralelo temos $\left|I_{\uparrow}\right|>\left|I_{\downarrow}\right|$ independentemente do sinal da tensão. Note que o mesmo ocorre no caso $p_{L}=p_{R}$ [Fig.4.11(c)]. Com relação ao caso antiparalelo [painel (d)] temos que $\left|I_{\downarrow}\right|$ é ligeiramente maior do que $\left|I_{\uparrow}\right|$ quando $U=0$, o que contrasta com o caso $p_{L}=p_{R}$ onde obtemos $I_{\uparrow}=I_{\downarrow}$ para $U=0$ [veja Fig.4.11(d)].

$\mathrm{Na}$ presença de interação de Coulomb, as correntes $I_{\uparrow}$ e $I_{\downarrow}$ são suprimidas na faixa de tensão correspondentes ao bloqueio de Coulomb $(1 \lesssim|e V| \lesssim 3 \mathrm{meV})$. Nesse caso $\left(p_{L} \neq p_{R}\right)$ temos um bloqueio de Coulomb dependente de spin em ambos os alinhamentos magnéticos $\mathrm{P}$ e AP, ao contrário do caso $p_{L}=p_{R}$ em que o bloqueio de Coulomb dependente de spin se manifestava somente na configuração AP. Isso ocorre porque quando $p_{L} \neq p_{R}$ temos uma magnetização não nula do ponto quântico em ambas as configurações P e AP, o que resulta num bloqueio de Coulomb dependente de spin nos dois alinhamento.

Em particular no alinhamento AP [Fig.4.12(d)], temos que a supressão de $I_{\downarrow}$ é maior do que a de $I_{\uparrow}$ para $V>0$ e o oposto para $V<0$, em semelhança ao que ocorre no caso $p_{L}=p_{R}$ [Fig.4.11(d)]. Essa diferença nas supressões de $I_{\uparrow} \mathrm{e}$ $I_{\downarrow}$ resulta numa inversão do sinal da polarização da corrente, conforme veremos a seguir.

Nos painéis (e)-(f) temos os gráficos de $\wp$. No caso paralelo [painel (e)] vemos que a interação de Coulomb leva a uma supressão de $\wp$ no regime de bloqueio de Coulomb para $V>0$ e a uma intensificação de $\wp$ para $V<0$. Isso ocorre porque para tensões positivas a corrente $I_{\uparrow}$ é mais intensamente suprimida do que a corrente $I_{\downarrow}$, em virtude de termos $n_{\downarrow}>n_{\uparrow}$. Logo a polarização $\wp$ tende a diminuir. Já para tensões negativas, como $n_{\uparrow}>n_{\downarrow}$, a corrente $I_{\downarrow}$ é mais fortemente suprimida, o que tende a aumentar a polarização. No caso antiparalelo temos que a polarização se comporta semelhantemente ao caso $p_{L}=p_{R}[\operatorname{compare~Figs.4.11(f)~}$ e 4.12(f)]. Porém, no caso $p_{L} \neq p_{R}$ temos $\wp<0$ para $U=0$ independentemente da tensão. Na presença de interação de Coulomb, a polarização se intensifica no regime de bloqueio de Coulomb, tornando-se positiva para uma certa faixa de tensão $V>0$, e ainda mais negativa numa outra faixa de tensão $V<0$. 
paralelo
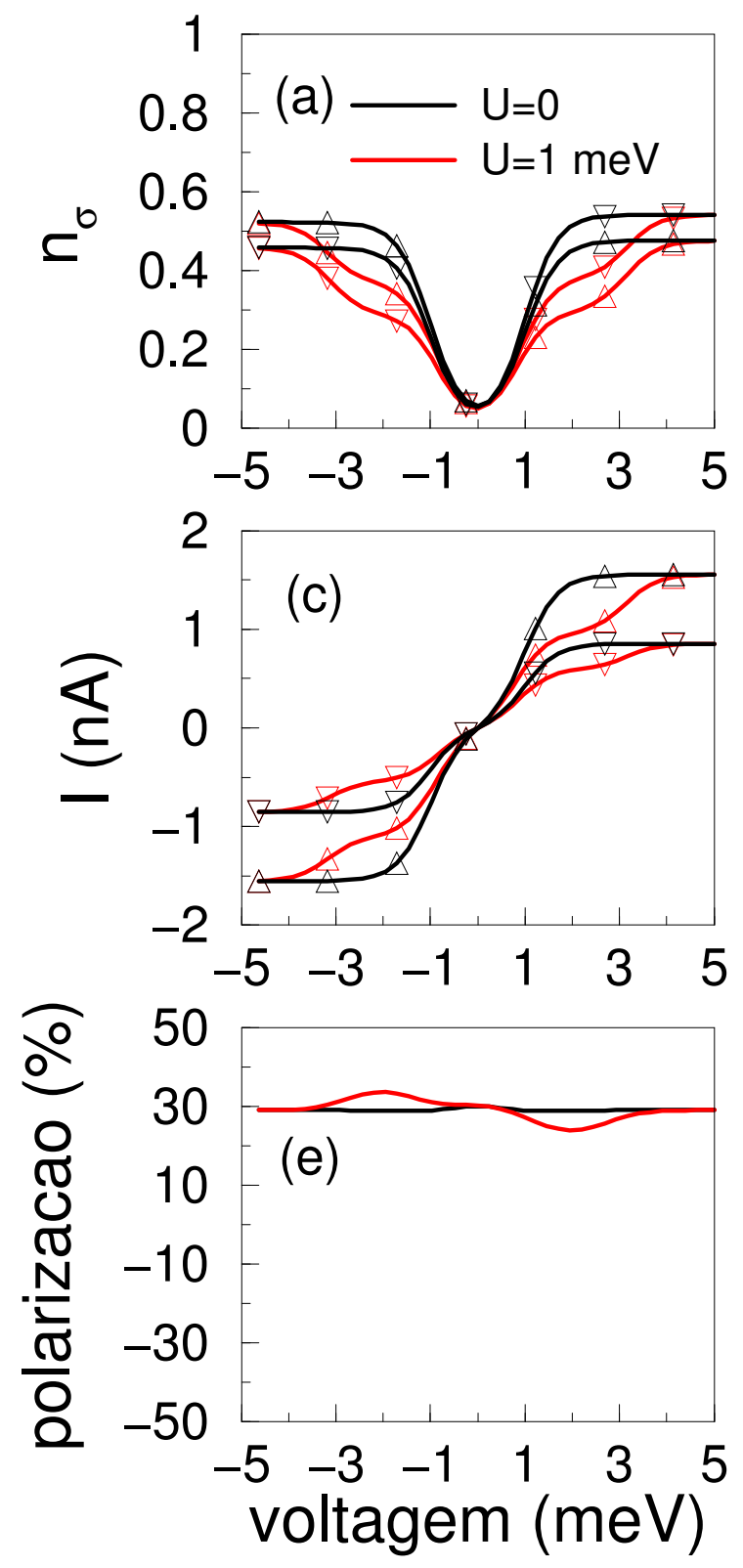

antiparalelo
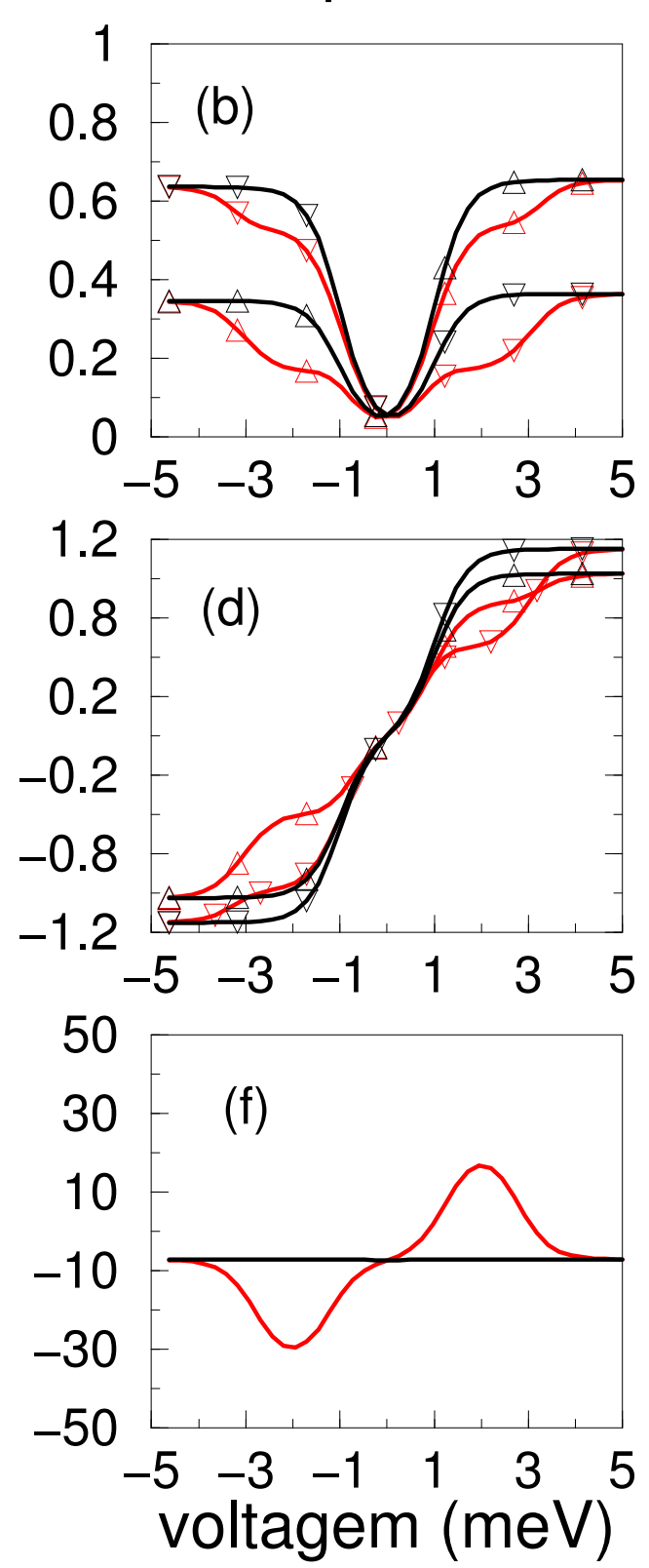

Figura 4.12: (a)-(b) Ocupações $n_{\uparrow}$ e $n_{\downarrow}$, (c)-(d) correntes $I_{\uparrow}$ e $I_{\downarrow}$, e (e)-(f) polarização nas configurações $\mathrm{P}$ e AP como função da voltagem para $p_{L}=0.23$ e $p_{R}=0.35$. As curvas para as ocupações $n_{\uparrow}$ e $n_{\downarrow}$ já foram graficadas na seção anterior para ambas as configurações. Com relação a corrente temos $\left|I_{\uparrow}\right|>\left|I_{\downarrow}\right|$ no alinhamento paralelo, o que se assemelha ao caso $p_{L}=p_{R}$ [Fig.4.11(c)]. Já no alinhamento antiparalelo temos $\left|I_{\downarrow}\right|>$ $\left|I_{\uparrow}\right|$, o que contrasta com o caso $p_{L}=p_{R}$, onde temos $I_{\uparrow}=I_{\downarrow}$ [Fig.4.11(d)]. A polarização da corrente no caso $\mathrm{P}$ apresenta uma supressão para $V>0$ e uma intensificação para $V<0$ devido ao bloqueio de Coulomb dependente de spin. No caso AP a polarização para $U=0$ é constante e negativa e na presença de interação de Coulomb sofre uma intensificação para uma certa faixa de tensão positiva (bloqueio de Coulomb) e uma supressão para tensões negativas. 


\subsection{Fator de supressão de corrente}

As supressões das correntes $I_{\uparrow}$ e $I_{\downarrow}$ observadas nas Figs.4.11 e 4.12, podem ser descritas através de um parâmetros que denominamos de fator de supressão. Um aspecto interessante desse fator é que o seu valor é o mesmo para as corrente $I_{\uparrow}$ e $I_{\downarrow}$ quando $n_{\uparrow}=n_{\downarrow}$, e diferente quando $n_{\uparrow} \neq n_{\downarrow}$. Portanto o fator de supressão nos diz se o bloqueio de Coulomb é ou não dependente de spin. Consideremos a diferença da corrente $I_{\sigma}$ com e sem interação de Coulomb, medida no ponto mediano do primeiro plateau da curva $I-V$ [em torno de $e V=2$ meV nas Figs.(4.11)-(4.12)],

$$
\begin{aligned}
& \Delta I_{\uparrow}=I_{\uparrow}^{U=0}-I_{\uparrow}^{U=1} \\
& \Delta I_{\downarrow}=I_{\downarrow}^{U=0}-I_{\downarrow}^{U=1},
\end{aligned}
$$

onde $I_{\sigma}^{U=1}$ é a corrente $\sigma$ medida no ponto central do plateau $(e V \approx 2 \mathrm{meV})$ com interação de Coulomb, e $I_{\sigma}^{U=0}$ a corrente no caso $U=0$ medida nesse mesmo ponto. Definimos o fator de supressão $\alpha_{\sigma}$ através da seguinte relação

$$
\begin{aligned}
& I_{\uparrow}^{U=1}=\frac{I_{\uparrow}^{U=0}}{\alpha_{\uparrow}}, \\
& I_{\downarrow}^{U=1}=\frac{I_{\downarrow}^{U=0}}{\alpha_{\downarrow}},
\end{aligned}
$$

onde dizemos que a corrente no ponto central do primeiro plateau ( $\mathrm{eV} \approx 2 \mathrm{meV}$ ), na presença de interação de Coulomb, é uma fração da corrente no caso $U=0$. Com isso a supressão $\Delta I_{\sigma}$ fica

$$
\begin{aligned}
& \Delta I_{\uparrow}=I_{\uparrow}^{U=0}-\frac{I_{\uparrow}^{U=0}}{\alpha_{\uparrow}}=I_{\uparrow}^{U=0}\left(1-\frac{1}{\alpha_{\uparrow}}\right) \\
& \Delta I_{\downarrow}=I_{\downarrow}^{U=0}-\frac{I_{\downarrow}^{U=0}}{\alpha_{\downarrow}}=I_{\downarrow}^{U=0}\left(1-\frac{1}{\alpha_{\downarrow}}\right)
\end{aligned}
$$

No caso $p_{L}=p_{R}$ e configuração paralela, vemos na Fig.(4.11) que o valor médio da corrente em $e V \approx 2 \mathrm{meV}$ vale $I_{\uparrow}=1.62 \mathrm{nA}$, e $I_{\downarrow}=0.70 \mathrm{nA}$. Na presença de interação de Coulomb esses valores médios diminuem [veja Fig.(4.11)], indo para $I_{\uparrow}=1.13 \mathrm{nA}$ e $I_{\downarrow}=0.49 \mathrm{nA}$ (sempre medindo em torno de $e V=2 \mathrm{meV}$ ). Com isso obtemos $\Delta I_{\uparrow}=0.49 \mathrm{nA}$ e $\Delta I_{\downarrow}=0.21 \mathrm{nA}$. Agora fazendo a razão $\Delta I_{\sigma} / I_{\sigma}^{U=0}$ encontramos

$$
\frac{\Delta I_{\uparrow}}{I_{\uparrow}^{U=0}}=\frac{\Delta I_{\downarrow}}{I_{\downarrow}^{U=0}}=0.30 .
$$

Como $\left(1-\frac{1}{\alpha_{\sigma}}\right)=\frac{\Delta I_{\sigma}}{I_{\sigma}^{U=0}}$, obtemos $\alpha_{\uparrow}=\alpha_{\downarrow}=1.43$. Isso mostra que o fator de supressão é o mesmo para ambas as componentes de spin. Já para o caso $p_{L}=0.23$ e $p_{R}=0.35$ (e configuração paralela) temos os seguintes valores das correntes na região do plateau positivo $(e V \approx 2 \mathrm{meV}): I_{\uparrow}^{U=0}=1.50 \mathrm{nA}, I_{\downarrow}^{U=0}=0.83 \mathrm{nA}$, $I_{\uparrow}^{U=1}=0.97 \mathrm{nA}$, e $I_{\downarrow}^{U=1}=0.60 \mathrm{nA}$. Esses valores levam a $\alpha_{\uparrow}=1.55$ e $\alpha_{\downarrow}=1.38$, o que nos mostra que a supressão da corrente $\uparrow$ é maior do que da corrente $\downarrow$. Já as 
correntes na faixa do plateau negativo $(e V \approx-2 \mathrm{meV})$ valem: $I_{\uparrow}^{U=0}=-1.56 \mathrm{nA}$, $I_{\downarrow}^{U=0}=-0.82 \mathrm{nA}, I_{\uparrow}^{U=1}=-1.15 \mathrm{nA}$, e $I_{\downarrow}^{U=1}=-0.57 \mathrm{nA}$. Com isso encontramos $\alpha_{\uparrow}=1.36$ e $\alpha_{\downarrow}=1.44$, de onde vemos que a supressão é mais intensa sobre a componente $\downarrow$ da corrente. Logo, para $V>0$ temos uma supressão maior da componente $\uparrow$ e para $V<0$ uma supressão maior sobre a componente $\downarrow$. Esse efeito deve-se ao bloqueio de Coulomb dependente de spin, que tende a suprimir mais uma das componentes de spin.

$\underline{\text { Em resumo, }}$ quando $\alpha_{\uparrow}=\alpha_{\downarrow}$ o bloqueio de Coulomb não depende do spin, e para $\alpha_{\uparrow} \neq \alpha_{\downarrow}$ temos um bloqueio de Coulomb dependente de spin.

\subsection{Corrente e magnetoresistência de tunelamento}

Nesta seção analisamos os efeitos da interação de Coulomb e do espalhamento de spin sobre a corrente total $I=I_{\uparrow}+I_{\downarrow}$ e a magnetoresistência de tunelamento (TMR). A TMR mede a variação de resistência do sistema quando passamos da configuração P para AP. Podemos definir a TMR da seguinte forma [32],

$$
T M R=\frac{I_{P}-I_{A P}}{I_{A P}},
$$

onde $I_{P}$ é a corrente na configuração paralela $(\mathrm{P})$ e $I_{A P}$ a corrente no alinhamento anti-paralelo (AP).

Na figura (4.13) mostramos o gráfico da corrente total e da magneto resistência de tunelamento (TMR) como função da tensão externa, variando o parâmetro de espalhamento de spin $R$. As setas verticais indicam o sentido de crescimento de $R$, cujos valores estão listados sobre o painel (c). Consideramos os casos $U=0$ e $U=1 \mathrm{meV}$, linhas pretas e vermelhas, respectivamente. Nessa figura adotamos o caso simétrico $p_{L}=p_{R}$.

Comparando as curvas em (a) e (b) vemos que a corrente no alinhamento AP é menor do que no caso paralelo. Esse efeito de variar o alinhamento magnético dos eletrodos e obter uma mudança na corrente é o que dá origem à magneto resistência. Quando o espalhamento de spin está presente, essa variação da corrente como função do alinhamento diminui, levando assim a uma supressão da TMR. Observamos que enquanto a corrente $I_{P}$ independe de $R$, a corrente $I_{A P}$ intensificase com $R$. Ressaltamos que para $R$ suficientemente grande temos $I_{A P}$ coincidindo com $I_{P}$. Como no numerador de (4.13) temos a diferença $I_{P}-I_{A P}$, obtemos uma supressão da TMR, com o aumento de $R$. Esse efeito também foi previsto teoricamente por Rudziński e Barnaś [32], via equações de taxa, em um sistema como o nosso (FM-PQ-FM). Também observamos que, na presença de interação de Coulomb, a corrente $I_{A P}$ converge mais rapidamente para $I_{P}$ com o aumento de $R$ [compare, por exemplo, o crescimento da corrente com e sem interação de Coulomb no ponto $e V=5 \mathrm{meV}$ ]. Isso indica que a interação de Coulomb renormaliza o 
parâmetro $R$ de modo a intensificar os efeitos do espalhamento de spin. Esse aspecto também pode ser entendido através de um modelo de interferência entre canais de condução, conforme discutiremos na próxima seção.
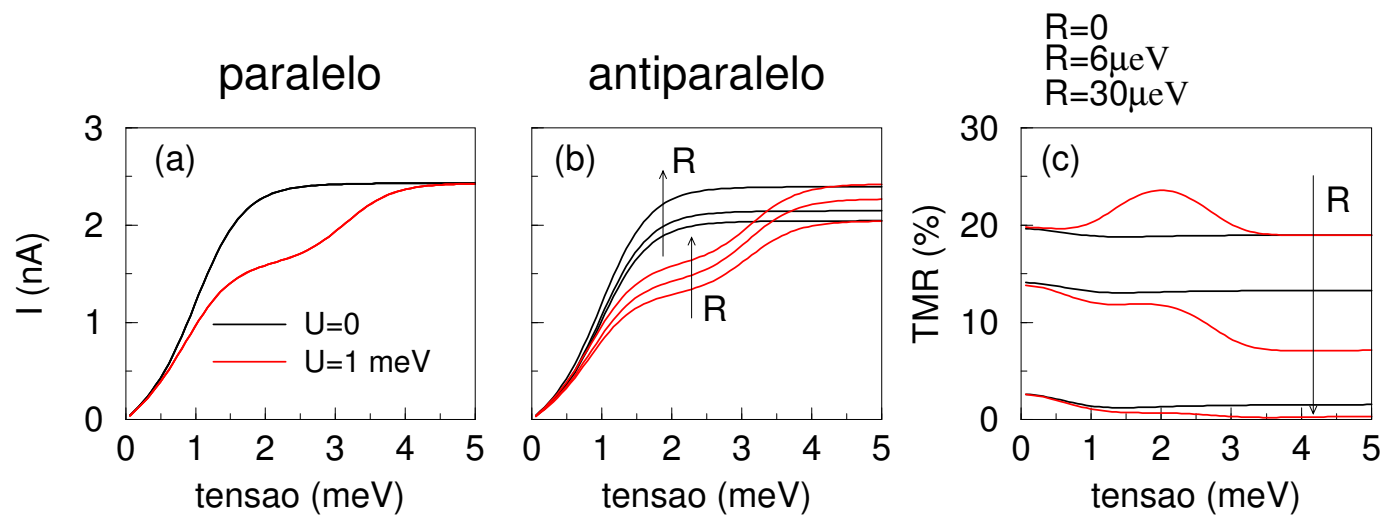

Figura 4.13: Correntes na configuração (a) paralela $I_{P}$, (b) anti-paralela $I_{A P}$ e (c) magneto-resistência de tunelamento TMR como função da tensão $V$ para diferentes valores de $R$ e de $U$. Enquanto a corrente na configuração $\mathrm{P}$ não varia com $R$ (as curvas para diferentes valores de $R$ estão superpostas), a corrente $I_{A P}$ aumenta com $R$, tendendo para o seu valor no caso paralelo. Esse aumento de $I_{A P}$ é mais rápido na presença de interação de Coulomb. O TMR intensifica-se no regime de bloqueio de Coulomb quando $R=0$. Na presença de espalhamento de spin o TMR diminui e essa supressão é mais intensa no caso $U=1 \mathrm{meV}$. Note, por exemplo, que para $U=1 \mathrm{meV}$ e $R=30 \mu \mathrm{eV}$ o TMR atinge valores próximos de zero.

A figura 4.13(c) mostra o gráfico do TMR, que foi obtido através das correntes $I_{P}$ e $I_{A P}$, ilustradas nas Figs.4.13(a)-(b). Para $R=0$ vemos que a interação de Coulomb intensifica a TMR no bloqueio de Coulomb [1 meV $\lesssim \mathrm{eV} \lesssim 3 \mathrm{meV}$. Isso ocorre porque a interação de Coulomb aumenta a resistência do sistema, dificultando a passagem de elétrons via bloqueio de Coulomb. Na presença de espalhamento de spin $(R=6 \mu \mathrm{eV}$ e $R=30 \mu \mathrm{eV})$ temos uma supressão da TMR. Em particular, para $R=30 \mu \mathrm{eV}$ e $U=1 \mathrm{meV}$ a TMR atinge valores próximos de zero. Note que a supressão da TMR, devido a espalhamento de spin, é maior na presença de interação de Coulomb (as curvas para $U=1 \mathrm{meV}$ estão abaixo das curvas para $U=0$ quando $R \neq 0$ ). Isso mostra que a interação de Coulomb, combinada com o espalhamento de spin, leva a uma supressão adicional da TMR. Esse efeito está de acordo com o fato da corrente $I_{A P}$ crescer mais rápido com $R$ na presença de interação de Coulomb, conforme mencionamos anteriormente. Uma explicação para esse efeito combinado da interação de Coulomb e do espalhamento de spin está discutido na seção seguinte.

Devemos ressaltar que a supressão da TMR devido a espalhamento de spin foi observado experimentalmente em uma junção do tipo FM-I-FM (I=isolante), 
mais especificamente $\mathrm{Co} / \mathrm{Al}_{2} \mathrm{O}_{3} / \mathrm{NiFe}$. Note que nesse sistema ao invés de um ponto quântico na região central tem-se uma barreira de tunelamento. Átomos de $\mathrm{Co}, \mathrm{Pd}, \mathrm{Cu}$ e $\mathrm{Ni}$ foram adicionados ao óxido de modo a servirem como centros espalhadores de spin. Os autores observaram que, com o aumento da espessura da barreira, a magneto resistência de tunelamento decrescia. Essa supressão foi atribuída ao espalhamento de spin, que se intensifica com o aumento dessa espessura. Rudziński e Barnaś [32] também obtiveram teoricamente a supressão da TMR devido a espalhamento de spin no sistema FM-PQ-FM, porém, a abordagem nesse caso foi em termos de equações de taxa.

\subsection{Efeitos de Interferência}

Aqui derivamos uma equação para o coeficiente de transmissão através do sistema FM-PQ-FM que tem a forma de uma equação de interferência entre amplitudes de probabilidade de tunelamento. Com isso conseguimos explicar a supressão adicional da TMR quando a interação de Coulomb e o espalhamento de spin estão presentes simultaneamente.

\subsubsection{Dedução de uma expressão analítica para a corrente}

$$
(U=0) \text {. }
$$

O espalhamento de spin dentro do ponto quântico dá origem aos canais de condução $\epsilon_{1}=\epsilon_{0}-R$ e $\epsilon_{2}=\epsilon_{0}+R$, isto é, o canal $\epsilon_{0}$ se desdobra nos canais $\epsilon_{1}$ e $\epsilon_{2}$, conforme vimos no Cap.3. Podemos mostrar que esses dois níveis se interferem quando estão energeticamente próximos entre si, o que nos auxilia na compreensão dos resultados da Fig.(4.13). Por simplicidade de argumento, consideramos a configuração antiparalela, $100 \%$ polarizada $\left(p_{L}=p_{R}=1\right), U=0$ e tensão $V$ grande o suficiente para que os canais $\epsilon_{1}$ e $\epsilon_{2}$ estejam dentro da janela de condução (i.e., $\mu_{R}<\epsilon_{i}<\mu_{L}$, $i=1,2)$

Para esses parâmetros, podemos deduzir uma expressão analítica para $I$ a partir da seguinte equação [Eq.(3.24)],

$$
I_{L}=\frac{i e}{\hbar} \int \frac{d \epsilon}{2 \pi} \operatorname{Tr}\left\{\boldsymbol{\Gamma}^{L}\left\{\left[\mathbf{G}^{r}(\epsilon)-\mathbf{G}^{a}(\epsilon)\right] n_{L}(\epsilon)+\mathbf{G}^{<}(\epsilon)\right\}\right\} .
$$

A matriz $\Gamma^{L}$ no caso $100 \%$ polarizado tem o seguinte aspecto

$$
\Gamma^{L}=\frac{1}{2}\left(\begin{array}{cc}
\Gamma_{\uparrow}^{L}+\Gamma_{\downarrow}^{L} & \Gamma_{\uparrow}^{L}-\Gamma_{\downarrow}^{L} \\
\Gamma_{\uparrow}^{L}-\Gamma_{\downarrow}^{L} & \Gamma_{\uparrow}^{L}+\Gamma_{\downarrow}^{L}
\end{array}\right)=\Gamma_{0}\left(\begin{array}{cc}
1 & 1 \\
1 & 1
\end{array}\right),
$$

visto que $\Gamma_{\uparrow}^{L}=\Gamma_{0}\left(1+p_{L}\right)=2 \Gamma_{0}$, e $\Gamma_{\downarrow}^{L}=\Gamma_{0}\left(1-p_{L}\right)=0$ no caso de $p_{L}=1$. Com isso a equação da corrente torna-se

$$
I_{L}=\frac{i e}{\hbar} \int \frac{d \epsilon}{2 \pi} \Gamma_{0} \operatorname{Tr}\left\{\left(\begin{array}{ll}
1 & 1 \\
1 & 1
\end{array}\right)\left[\mathbf{G}^{<}+n_{L}\left(\mathbf{G}^{r}-\mathbf{G}^{a}\right)\right]\right\} .
$$


A equação para $\mathbf{G}^{<}$é obtida a partir da equação de Keldysh,

$$
\begin{aligned}
& \mathbf{G}^{<}=\mathbf{G}^{r} \boldsymbol{\Sigma}^{<} \mathbf{G}^{a}= \mathbf{G}^{r}\left[\frac{i n_{L}}{2}\left(\begin{array}{cc}
\Gamma_{\uparrow}^{L}+\Gamma_{\downarrow}^{L} & \Gamma_{\uparrow}^{L}-\Gamma_{\downarrow}^{L} \\
\Gamma_{\uparrow}^{L}-\Gamma_{\downarrow}^{L} & \Gamma_{\uparrow}^{L}+\Gamma_{\downarrow}^{L}
\end{array}\right)+\right. \\
&\left.\frac{i n_{R}}{2}\left(\begin{array}{cc}
\Gamma_{\uparrow}^{R}+\Gamma_{\downarrow}^{R} & \Gamma_{\uparrow}^{R}-\Gamma_{\downarrow}^{R} \\
\Gamma_{\uparrow}^{R}-\Gamma_{\downarrow}^{R} & \Gamma_{\uparrow}^{R}+\Gamma_{\downarrow}^{R}
\end{array}\right)\right] \mathbf{G}^{a} \\
&=\mathbf{G}^{r} \frac{i}{2}\left[n_{L} 2 \Gamma_{0}\left(\begin{array}{cc}
1 & 1 \\
1 & 1
\end{array}\right)+n_{R} 2 \Gamma_{0}\left(\begin{array}{cc}
1 & -1 \\
-1 & 1
\end{array}\right)\right] \mathbf{G}^{a} .(1
\end{aligned}
$$

Para tensões grandes o suficiente temos $n_{L}=1$ e $n_{R}=0$, de modo que a equação acima simplifica em

$$
\mathbf{G}^{<}=i \Gamma_{0} \mathbf{G}^{r}\left(\begin{array}{ll}
1 & 1 \\
1 & 1
\end{array}\right) \mathbf{G}^{a} .
$$

Para a função de Green retardada utilizamos as Eqs.(3.65) e (3.70), o que nos leva a

$$
\begin{aligned}
\mathbf{G}^{r} & =\left(\mathbf{G}^{0^{-1}}-\boldsymbol{\Sigma}^{r}\right)^{-1} \\
& =\left(\left(\begin{array}{cc}
\epsilon-\epsilon_{1} & 0 \\
0 & \epsilon-\epsilon_{2}
\end{array}\right)+\frac{i}{4}\left(\begin{array}{cc}
2 \Gamma_{0} & 2 \Gamma_{0} \\
2 \Gamma_{0} & 2 \Gamma_{0}
\end{array}\right)+\frac{i}{4}\left(\begin{array}{cc}
2 \Gamma_{0} & -2 \Gamma_{0} \\
-2 \Gamma_{0} & 2 \Gamma_{0}
\end{array}\right)\right)^{-1} \\
& =\left(\begin{array}{cc}
\frac{1}{\epsilon-\epsilon_{1}+i \Gamma_{0}} & 0 \\
0 & \frac{1}{\epsilon-\epsilon_{2}+i \Gamma_{0}}
\end{array}\right)
\end{aligned}
$$

Com isso a diferença $\mathbf{G}^{r}-\mathbf{G}^{a}$ vale

$$
\mathbf{G}^{r}-\mathbf{G}^{a}=\left(\begin{array}{cc}
\frac{-2 i \Gamma_{0}}{\left(\epsilon-\epsilon_{1}\right)^{2}+\Gamma_{0}^{2}} & 0 \\
0 & \frac{-2 i \Gamma_{0}}{\left(\epsilon-\epsilon_{2}\right)^{2}+\Gamma_{0}^{2}}
\end{array}\right) .
$$

Substituindo as Eqs.(4.18) e (4.20) na expressão da corrente (4.16) encontramos

$$
\begin{aligned}
I_{L}= & \frac{i e}{\hbar} \int \frac{d \epsilon}{2 \pi} i \Gamma_{0}^{2} \operatorname{Tr}\left\{\left(\begin{array}{ll}
1 & 1 \\
1 & 1
\end{array}\right) \times\right. \\
& {\left[\left(\begin{array}{cc}
\frac{1}{\epsilon-\epsilon_{1}+i \Gamma_{0}} & 0 \\
0 & \frac{1}{\epsilon-\epsilon_{2}+i \Gamma_{0}}
\end{array}\right)\left(\begin{array}{ll}
1 & 1 \\
1 & 1
\end{array}\right)\left(\begin{array}{cc}
\frac{1}{\epsilon-\epsilon_{1}-i \Gamma_{0}} & 0 \\
0 & \frac{1}{\epsilon-\epsilon_{2}-i \Gamma_{0}}
\end{array}\right)-\right.} \\
& \left.2\left(\begin{array}{cc}
\frac{1}{\left(\epsilon-\epsilon_{1}\right)^{2}+\Gamma_{0}^{2}} & 0 \\
0 & \frac{1}{\left(\epsilon-\epsilon_{2}\right)^{2}+\Gamma_{0}^{2}}
\end{array}\right)\right] .
\end{aligned}
$$

Após uma série de multiplicações matriciais obtemos

$$
\begin{aligned}
I_{L}= & \frac{e \Gamma_{0}^{2}}{\hbar} \int \frac{d \epsilon}{2 \pi}\left\{\frac{1}{\left(\epsilon-\epsilon_{1}\right)^{2}+\Gamma_{0}^{2}}+\frac{1}{\left(\epsilon-\epsilon_{2}\right)^{2}+\Gamma_{0}^{2}}\right. \\
& \left.-\frac{1}{\left(\epsilon-\epsilon_{1}-i \Gamma_{0}\right)\left(\epsilon-\epsilon_{2}+i \Gamma_{0}\right)}-\frac{1}{\left(\epsilon-\epsilon_{1}+i \Gamma_{0}\right)\left(\epsilon-\epsilon_{2}-i \Gamma_{0}\right)}\right\} .
\end{aligned}
$$


Definindo as amplitudes $t_{\epsilon_{1}}=\frac{1}{\epsilon-\epsilon_{1}+i \Gamma_{0}}$ e $t_{\epsilon_{2}}=\frac{1}{\epsilon-\epsilon_{2}+i \Gamma_{0}}$, a corrente fica

$$
I_{L}=\frac{e \Gamma_{0}^{2}}{\hbar} \int \frac{d \epsilon}{2 \pi}\left\{\left|t_{\epsilon_{1}}\right|^{2}+\left|t_{\epsilon_{2}}\right|^{2}-2 \operatorname{Re}\left(t_{\epsilon_{1}} t_{\epsilon_{2}}^{*}\right)\right\}
$$

de onde podemos definir um coeficiente de transmissão

$$
T=\Gamma_{0}^{2}\left\{\left|t_{\epsilon_{1}}\right|^{2}+\left|t_{\epsilon_{2}}\right|^{2}-2 \operatorname{Re}\left(t_{\epsilon_{1}} t_{\epsilon_{2}}^{*}\right)\right\}
$$

de modo a expressar a corrente assim:

$$
I=\frac{e}{h} \int T(\epsilon) d \epsilon
$$

que tem a mesma forma da equação de Landauer-Büttiker [64]. O coeficiente $T$ descreve a transmissão coerente através dos canais $\epsilon_{1}$ e $\epsilon_{2}$. O termo $2 \operatorname{Re}\left(t_{\epsilon_{1}} t_{\epsilon_{2}}^{*}\right)$ descreve a interferência entre esse canais. Note que para $R=0$ temos $\epsilon_{1}=$ $\epsilon_{0}-R=\epsilon_{0}$ e $\epsilon_{2}=\epsilon_{0}+R=\epsilon_{0}$, portanto $\epsilon_{1}=\epsilon_{2}$. Nesse caso $t_{\epsilon_{1}} t_{\epsilon_{2}}^{*}=\left|t_{\epsilon_{1}}\right|^{2}=\left|t_{\epsilon_{2}}\right|^{2}$, e com isso $\left|t_{\epsilon_{1}}\right|^{2}+\left|t_{\epsilon_{2}}\right|^{2}=2 \operatorname{Re}\left(t_{\epsilon_{1}} t_{\epsilon_{2}}^{*}\right)$, resultando num coeficiente de transmissão nulo $T=0$. Dizemos então que para $R=0$ ocorre uma interferência destrutiva máxima entre os canais $\epsilon_{1}$ e $\epsilon_{2}$ que resulta numa corrente nula. Vale ressaltar que, como os terminais são anti-paralelamente alinhados e $100 \%$ polarizados, só pode haver corrente na presença de espalhamento de spin. Portanto o resultado $T=0$ para $R=0$ era esperado. A seguir generalizamos essas idéias para o caso $U \neq 0$.

\subsubsection{Caso $U \neq 0$}

Quando há interação de Coulomb, além dos canais $\epsilon_{1}$ e $\epsilon_{2}$ discutidos acima, temos também os canais $\epsilon_{1}+U$ e $\epsilon_{2}+U$. Os efeitos gerados pela superposição dos canais $\epsilon_{1}+U$ e $\epsilon_{2}$ podem ser modelados qualitativamente através de um coeficiente de transmissão semelhante ao dado na Eq.(4.24).

O painel superior da figura (4.14) ilustra esquematicamente os quatro canais de condução $\epsilon_{1}, \epsilon_{2}, \epsilon_{1}+U$ e $\epsilon_{2}+U$, em três regimes de transporte: $U \ll \epsilon_{2}-\epsilon_{1}, U \approx \epsilon_{2}-$ $\epsilon_{1}$ e $U \gg \epsilon_{2}-\epsilon_{1}$, denotados por I, II e III, respectivamente. Cada um desses regimes possui características de transporte distintas, que serão analisadas no decorrer desta seção. Na Fig. 4.14(a) temos a função espectral $A(\epsilon)$ na configuração antiparalela para valores crescentes de $U$. Os casos I, II e III estão indicados nesse gráfico. Note que para $U=0$ temos apenas os picos $\epsilon_{1}$ e $\epsilon_{2}$ espaçados de $2 R$ (utilizamos $R=20 \mu \mathrm{eV}$ ). Com o aumento de $U$ os picos $\epsilon_{1}+U$ e $\epsilon_{2}+U$ se deslocam para a direita. Quando $U$ é da ordem do espaçamento entre os níveis $\epsilon_{1}$ e $\epsilon_{2}$ $\left(U \approx \epsilon_{2}-\epsilon_{1}\right)$ ocorre uma superposição dos picos $\epsilon_{1}+U$ e $\epsilon_{2}$ [veja região II].

Fazendo o gráfico da corrente $I_{A P}$ como função de $U$ para $R=20 \mu \mathrm{eV}$ [Fig.4.14(b)], obtida através da equação geral (3.24), observamos uma supressão (dip) de $I_{A P}$ quando os picos $\epsilon_{1}+U$ e $\epsilon_{2}$ se cruzam. Assumindo que o mesmo mecanismo que gerou a supressão da corrente no caso $U=0$ quando $\epsilon_{1}=\epsilon_{2}$, também gera a 
supressão da corrente quando $\epsilon_{1}+U=\epsilon_{2}$, escrevemos o seguinte coeficiente de transmissão

$$
T_{\epsilon_{1}+U, \epsilon_{2}}=\left|t_{\epsilon_{1}+U}\right|^{2}+\left|t_{\epsilon_{2}}\right|^{2}-2 \operatorname{Re}\left(t_{\epsilon_{1}+U} t_{\epsilon_{2}}^{*}\right),
$$

onde $t_{\widetilde{\epsilon}}=1 /\left(\epsilon-\widetilde{\epsilon}+i \Gamma_{0}\right)$, sendo $\widetilde{\epsilon}=\epsilon_{1}+U$ ou $\epsilon_{2}$. Vale salientar que não temos uma expressão analítica para o coeficiente de transmissão na presença de interação de Coulomb, a Eq.(4.26) é um ansatz motivado pela Eq.(4.24). A integral $I_{\epsilon_{1}+U, \epsilon_{2}}=\int T_{\epsilon_{1}+U, \epsilon_{2}}(\epsilon) d \epsilon$, que é semelhante a Eq.(4.23), está graficada na Fig.4.14(c). Observe que $I_{\epsilon_{1}+U, \epsilon_{2}}$ tem um mínimo quando $\epsilon_{1}+U=\epsilon_{2}$, semelhantemente ao cálculo numérico [Fig.4.14(b)]. Portanto, conseguimos reproduzir qualitativamente, através da Eq.(4.26), o comportamento da corrente $I_{A P}$. 


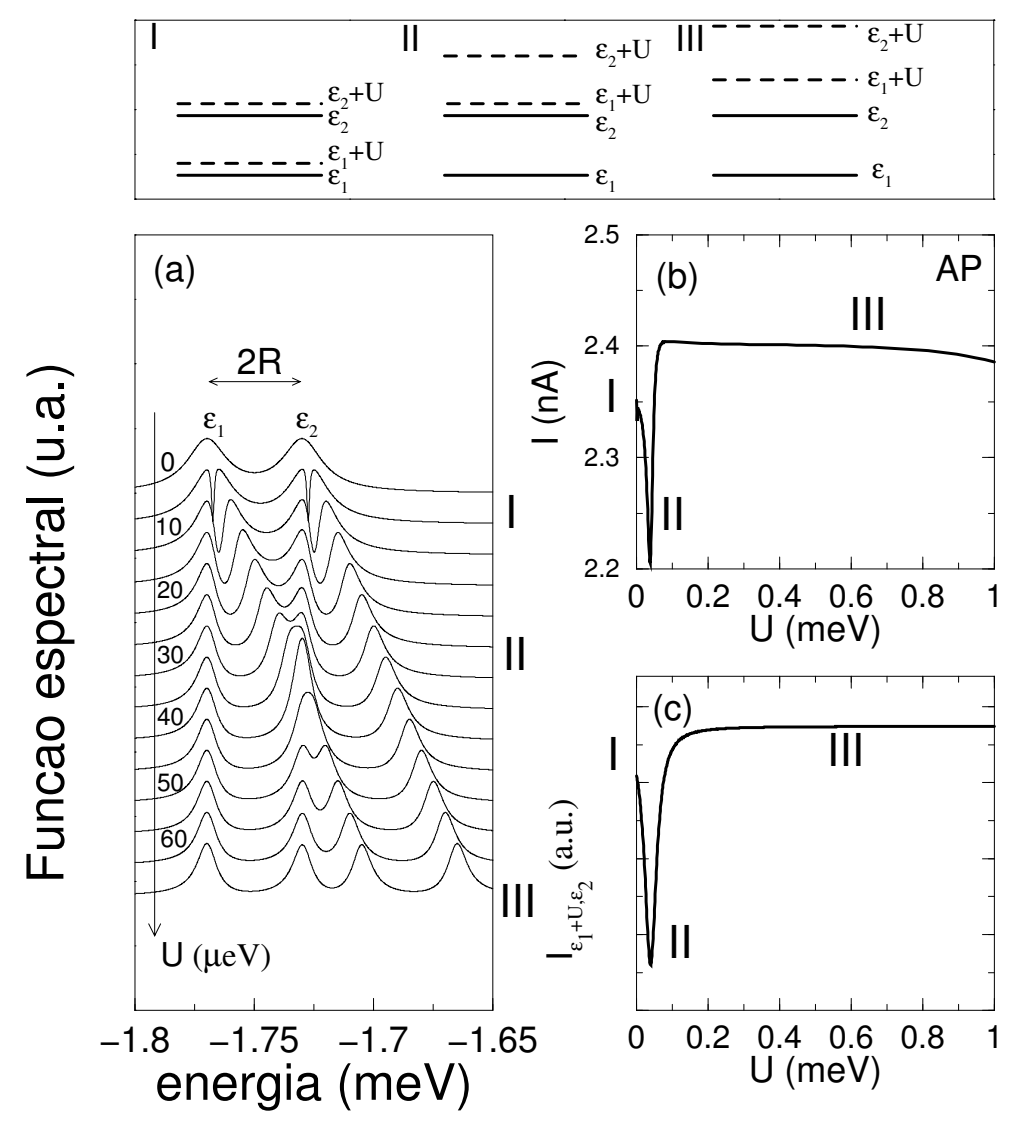

Figura 4.14: (a) Função espectral $A(\epsilon)$, (b) corrente anti-paralela $I_{A P}$ (cálculo numérico) e (c) corrente no modelo de interferência $I_{\epsilon_{1}+U, \epsilon_{2}}$ como função de $U$. No topo ilustramos os vários canais de condução $\epsilon_{1}, \epsilon_{2}, \epsilon_{1}+U$ e $\epsilon_{2}+U$ em três regimes distintos: (I) $U \simeq 0$, (II) $U \simeq\left(\epsilon_{2}-\epsilon_{1}\right)$ e (III) $U>>\left(\epsilon_{2}-\epsilon_{1}\right)$. Esses regimes também estão indicados nos demais painéis. Na função espectral vemos que os picos $\epsilon_{1}+U$ e $\epsilon_{2}+U$ deslocam-se para a direita a medida que $U$ aumenta. Em particular, para $U=40 \mu \mathrm{eV}$ temos que $\epsilon_{1}+U=\epsilon_{2}$ [região II]. Nessa condição a corrente apresenta uma supressão [veja região II no painel (b)]. Além disso a corrente é maior em III do que em I, isto é, para um $R$ fixo $(R=20 \mu \mathrm{eV})$ a corrente $I_{A P}$ é maior para $U=1 \mathrm{meV}$ do que para $U=0$. Reproduzimos qualitativamente o comportamento da corrente $I_{A P}$ [painel (b)] através da função $I_{\epsilon_{1}+U, \epsilon_{2}}$, que descreve a interferência entre os canais $\epsilon_{1}+U$ e $\epsilon_{2}$. Isso indica que o mínimo da corrente $I_{A P}$ observado em II e a sua diferença entre os limites I e III devese a uma interferência entre os canais do ponto quântico. Adotamos $p_{L}=p_{R}=p=0.4$, $\Gamma_{0}^{L}=\Gamma_{0}^{R}=10 \mu \mathrm{eV}, k_{B} T=0.17 \mathrm{meV}, R=20 \mu \mathrm{eV}, \mathrm{eV}=4 \mathrm{meV}$. 
Assim como no caso $U=0$, onde o cruzamento dos canais $\epsilon_{1}$ e $\epsilon_{2}$ corresponde a uma supressão do espalhamento de spin $(R=0)$, o cruzamento dos canais $\epsilon_{1}+U$ e $\epsilon_{2}$ também tende a suprimir o espalhamento de spin. Esse efeito, além de estar indicado pela corrente $I_{A P}$ que apresenta um mínimo quando $\epsilon_{1}+U=\epsilon_{2}$, também aparece na função espectral $A(\epsilon)$ quando o sistema está alinhado paralelamente. Na figura (4.5) observamos o aparecimento de um pico estreito na função espectral quando $\epsilon_{1}+U=\epsilon_{2}$. Esse pico estreito deve-se ao nível $\epsilon_{\downarrow}$ que tende a se desacoplar dos terminais quando $\epsilon_{1}+U=\epsilon_{2}$ em virtude da supressão do espalhamento de spin quando $\epsilon_{1}+U=\epsilon_{2}$.

Um outro aspecto relevante é o fato da corrente $I_{A P}$ ser maior para $U=1$ do que para $U=0$, conforme vemos na figura 4.14(b). Podemos entender isso notando que para $U$ pequeno $(U \rightarrow 0)$, os picos $\epsilon_{1}+U$ e $\epsilon_{2}$ estão mais próximos entre si do que no caso $U=1 \mathrm{meV}$. Assim, há uma interferência maior entre esses canais quando $U \rightarrow 0$, resultando numa maior supressão da corrente $I_{A P}$ para $U=0$ do que para $U=1 \mathrm{meV}$. Ou em outras palavras, quando $U=1 \mathrm{meV}$ podemos dizer que o espalhamento de spin fica mais eficaz.

$T M R$. Sendo a corrente $I_{A P}$ maior para $U=1 \mathrm{meV}$ do que para $U=0$, a magneto resistência de tunelamento tende a ser menor para $U=1 \mathrm{meV}$ do que para $U=0$, visto que $T M R=\left(I_{P}-I_{A P}\right) / I_{A P}$. Isso está de acordo com a supressão adicional do $T M R$ na presença de espalhamento de spin e interação de Coulomb observada na veja Fig. 4.13(c).

\subsection{Modelando o espalhamento de spin através de uma associação de resistores}

O problema de dupla barreira com tunelamento ressonante pode ser descrito fenomenologicamente através de um circuito elétrico, em que cada resistor corresponde a uma barreira de tunelamento [16]. Aqui estenderemos essa fenomenologia para o espalhamento de spin, e mostraremos que seus efeitos sobre a corrente podem ser reproduzidos qualitativamente quando associamos um resistor ao parâmetro de espalhamento de spin $R$.

A figura (4.15) ilustra um arranjo de resistores equivalente ao sistema FMPQ-FM. Nessa figura temos que as resistências $r_{\uparrow}^{L}$ e $r_{\downarrow}^{L}$ estão relacionadas com as taxas de tunelamento $\Gamma_{\uparrow}^{L}$ e $\Gamma_{\downarrow}^{L}$, enquanto as resistências $r_{\uparrow}^{R}$ e $r_{\downarrow}^{R}$ equivalem as taxas $\Gamma_{\uparrow}^{R}$ e $\Gamma_{\downarrow}^{R}$, respectivamente. O ramo superior, dado pelas resistências $r_{\uparrow}^{L} \mathrm{e}$ $r_{\uparrow}^{R}$, corresponde ao canal para spins $\uparrow$, e o ramo inferior (resistências $r_{\downarrow}^{L}$ e $r_{\downarrow}^{R}$ ) ao canal para spins $\downarrow$. A resistência intermediária $r$ acopla o ramo superior ao ramo inferior, fazendo o papel do espalhamento de spin nesta descrição. Não levamos em conta aqui interação de Coulomb. Para se descrever os efeitos de Coulomb nessa visão de circuito elétrico, costuma-se utilizar capacitores associados em paralelo às 
resistências [16],[84].

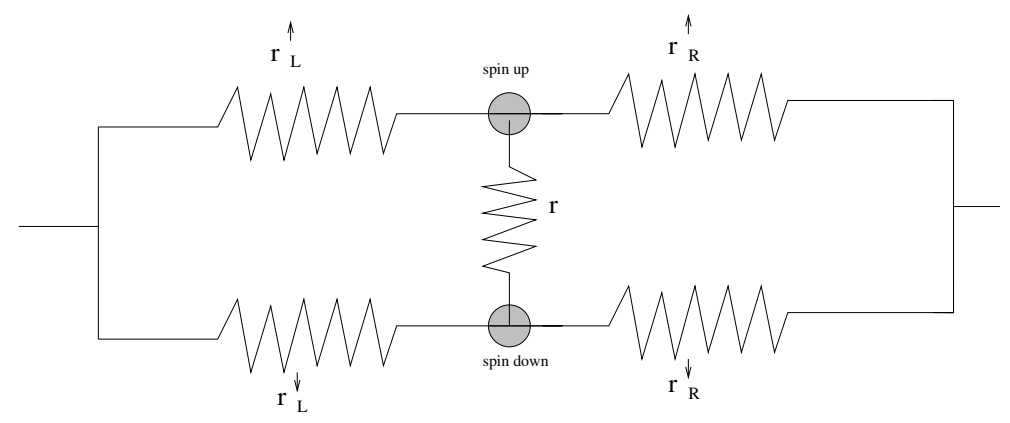

Figura 4.15: Associação de resistores que modela qualitativamente os efeitos do espalhamento de spin sobre a corrente. Os resistores $r_{\eta}^{\sigma}$ correspondem aos parâmetros de acoplamento $\Gamma_{\sigma}^{\eta}$, e o resistor $r$ equivale ao parâmetro de espalhamento de spin $R$. Para $r \rightarrow \infty$ temos que o ramo superior se desacopla do ramo inferior, logo $r$ é inversamente proporcional a $R$. A medida que $r$ diminui (ou $R$ aumenta) a resistência equivalente do circuito diminui, resultando assim num aumento da corrente.

A configuração magnética paralela é obtida adotando-se $r_{L}^{\uparrow}=r_{R}^{\uparrow}$ e $r_{L}^{\downarrow}=r_{R}^{\downarrow}$. Isso é equivalente a $\Gamma_{\sigma}^{L}=\Gamma_{\sigma}^{R}$. Para a configuração anti-paralela temos $r_{\uparrow}^{L}=r_{\downarrow}^{R}$ e $r_{L}^{\downarrow}=$ $r_{R}^{\uparrow}$, de maneira equivalente a $\Gamma_{\uparrow}^{L}=\Gamma_{\downarrow}^{R}$ e $\Gamma_{\downarrow}^{L}=\Gamma_{\uparrow}^{R}$. Eliminamos o "espalhamento de spin" nessa associação de resistores tomando o limite $r \rightarrow \infty$, de modo a desacoplar o ramo superior do ramo inferior. Temos então que a resistência $r$ e o parâmetro de espalhamento de spin são inversamente proporcionais, isto é, para $R=0$ (espalhamento de spin nulo) temos $r \rightarrow \infty$.

Considerando uma tensão externa $V$, obtemos a seguinte expressão para a corrente no sistema da Fig.(4.15)

$$
I=\frac{r^{2}}{r^{2}-\widetilde{r}^{\uparrow} \widetilde{r}^{\downarrow}}\left[\frac{\widetilde{r}^{\uparrow} \widetilde{r}^{\downarrow}}{r}\left(\frac{1}{r_{L}^{\uparrow} r_{R}^{\downarrow}}+\frac{1}{r_{L}^{\downarrow} r_{R}^{\uparrow}}\right)+\frac{\widetilde{r}^{\uparrow}}{r_{L}^{\uparrow} r_{R}^{\uparrow}}+\frac{\widetilde{r}^{\downarrow}}{r_{L}^{\downarrow} r_{R}^{\downarrow}}\right] V,
$$

onde $\frac{1}{\widetilde{r}_{\sigma}}=\frac{1}{r_{L}^{\sigma}}+\frac{1}{r_{R}^{\sigma}}+\frac{1}{r}$. Observe que, para $R=0(r \rightarrow \infty)$, obtemos

$$
I=\left(\frac{1}{r_{L}^{\uparrow}+r_{R}^{\uparrow}}+\frac{1}{r_{L}^{\downarrow}+r_{R}^{\downarrow}}\right) V
$$

que é um resultado esperado quando o segmento da resistência $r$ inexiste, isto é, quando se trata de um sistema de resistores equivalentes $r_{L}^{\uparrow}+r_{R}^{\uparrow}$ e $r_{L}^{\downarrow}+r_{R}^{\downarrow}$ em paralelo.

Ressaltamos que as Eqs.(4.27)-(4.28) corresponde a lei de Ohm, e portanto trata-se de resposta linear. Já os cálculos via funções de Green para o sistema físico real (FM-PQ-FM) são totalmente não lineares. Portanto, não podemos estudar a dependência da corrente como função de $V$ através dessa associação de resistores. Aqui $V$ entra simplesmente como um parâmetro que deve ser fixado em $e V=\Gamma_{0}$, 
para que possamos reganhar alguns resultados obtidos via funções de Green. Os resistores nessa visão entram simplesmente para descrever taxas de tunelamento.

Na figura 4.16 graficamos as correntes $I_{P}$ e $I_{A P}$ como função do parâmetro de espalhamento de spin $R$ (ou de $1 / r$ ) obtidas via o modelo de resistores [painel (a)] e através do cálculo numérico [painel (b)]. Para gerar a curvas do painel (a) utilizamos a seguinte parametrização

$$
\begin{aligned}
r_{\sigma}^{\eta} & =\frac{\hbar}{e^{2}} \frac{\Gamma_{0}}{\Gamma_{\sigma}^{\eta}} \\
r & =\frac{\hbar}{e^{2}} \frac{\Gamma_{0}}{R} .
\end{aligned}
$$

Observamos que $I_{P}$ independe do valor de $R$ [Fig.4.16(a)], assim como o resultado numérico obtido para o sistema FM-PQ-FM [Fig.4.16(b)]. Na configuração AP temos que $I_{A P}$ aumenta com $R$ (ou $1 / r$ ), tendendo para o seu valor na configuração P. Observamos o mesmo comportamento para a corrente $I_{A P}$ no sistema FM-PQFM (obtida via o formalismo de funções de Green).
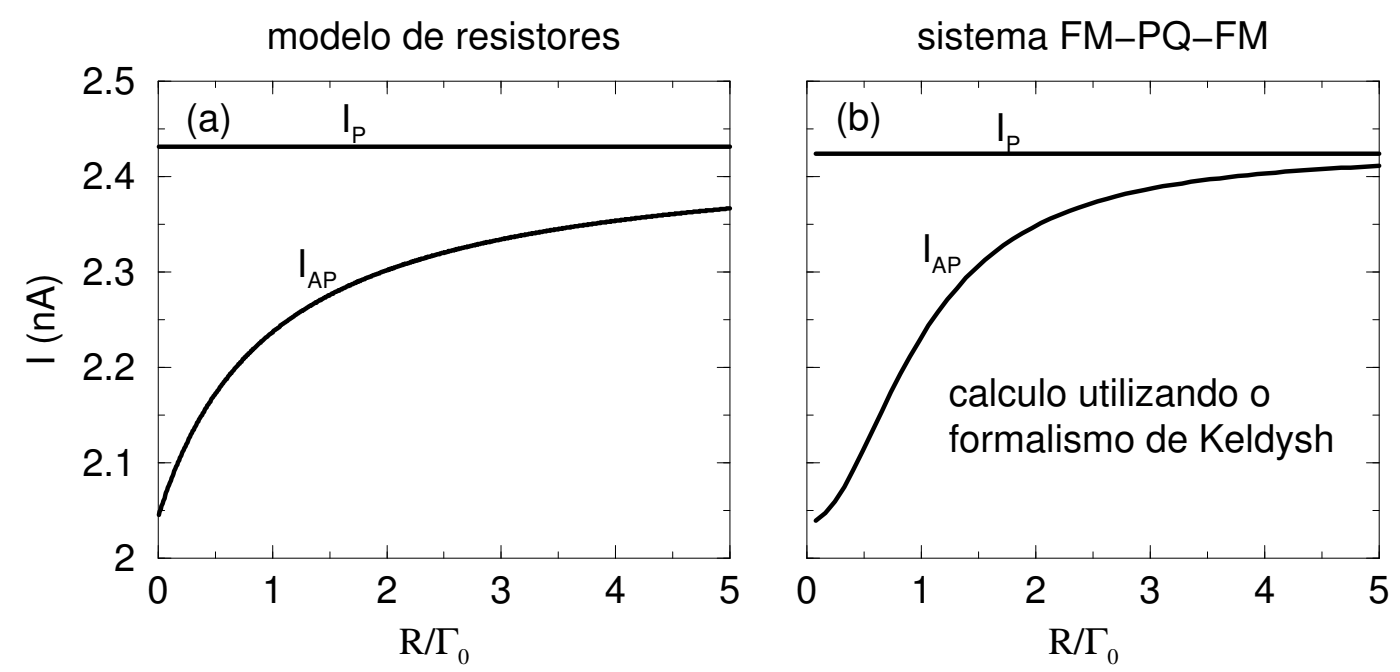

Figura 4.16: Correntes $I_{P}$ e $I_{A P}$ como função do parâmetro de espalhamento de spin $R$ para (a) a associação de resistores ilustrada na Fig.(4.15), e (b) o sistema FM-PQ-FM. Observamos que a corrente $I_{P}$ independe do parâmetro $R$ nos dois sistemas, enquanto a corrente $I_{A P}$ aumenta com $R$. O aumento da corrente $I_{A P} \operatorname{com} R$ deve-se a diminuição da resistência total do sistema. 


\subsection{Ruído}

Dedicamos essa última seção ao estudo do ruído no sistema FM-PQ-FM. Encontramos que o bloqueio de Coulomb dependente de spin também se manifesta nas curvas do ruído, resultando numa supressão maior de uma dada componente de spin. Também notamos que o espalhamento de spin, combinado com a polarização dos terminais ferromagnéticos, pode levar a uma forte supressão do ruído $(\gamma \approx 0.2)$, quando o sistema está na configuração magnética AP. Apresentamos ainda, através do cálculo do fator de Fano, uma analogia entre o nosso sistema, e o problema de tripla-barreira de tunelamento. Uma análise do ruído térmico também será apresentada.

\subsubsection{Efeitos do bloqueio de Coulomb sobre o ruído}

Caso simétrico $p_{L}=p_{R}$. Comecemos avaliando os efeitos do bloqueio de Coulomb dependente de spin sobre o ruído. A figura (4.17) mostra os gráficos das corrente $I_{\uparrow}$ e $I_{\downarrow}$ [painéis (a)-(b)] e dos seus respectivos ruídos $S_{\uparrow}$ e $S_{\downarrow}$ [painéis (c)-(d)] contra tensão para as configurações $\mathrm{P}$ e $\mathrm{AP}$, e caso $p_{L}=p_{R}$. Os gráficos de corrente já foram apresentados anteriormente, e estão reproduzidos aqui para efeitos de comparação com o ruído.

Para $U=0$ temos $S_{\uparrow}>S_{\downarrow}$ no alinhamento paralelo [painel (c)] e $S_{\uparrow}=S_{\downarrow}$ no caso anti-paralelo [painel (d)], assim como se dá com a corrente. Quando ligamos a interação de Coulomb $(U=1 \mathrm{meV})$, o ruído passa a ser suprimido no regime de bloqueio de Coulomb. Essa supressão depende do spin (bloqueio de Coulomb dependente de spin), sendo maior sobre a componente de spin $\downarrow$ no alinhamento AP [veja Fig.4.17(d)]. Efeito semelhante ocorre sobre a corrente [Fig.4.17(b)], conforme já discutimos anteriormente. No alinhamento paralelo [Fig.4.17(c)], temos a falsa impressão de que o ruído $S_{\uparrow}$ é mais fortemente suprimido do que o ruído $S_{\downarrow}$. Porém, quando medimos a supressão relativa (fator de supressão) de ambas as componentes de spin, vemos que as suas supressões são iguais. Essa análise está feita na seção seguinte.

Um outro aspecto interessante é o declive negativo observado na curva $S_{\downarrow}$ para a configuração AP [Fig. 4.17(d)]. Declive semelhante ocorre também em algumas outras curvas, como por exemplo na curva de $I_{\downarrow}$ e configuração AP. Porém, devido a efeitos térmicos $\left(k_{B} T\right)$, essa inclinação é mais evidente na curva de $S_{\downarrow}$ do que na de $I_{\downarrow}$. Esse efeito está relacionado a uma competição entre o aumento da transmissão do sistema, a medida que o nível $\epsilon_{0}$ começa a entrar em ressonância, e o bloqueio de Coulomb. Em outras palavras, quando o nível $\epsilon_{0}$ começa a entrar em ressonância (alinhando-se ao potencial químico do emissor), a transmissão por esse canal aumenta. Com resultado disso a corrente e o ruído também aumentam. Por outro lado, o nível $\epsilon_{0}$ se popula mais a medida em que vai se alinhando ao potencial 
químico do emissor, resultando no bloqueio de Coulomb que tende a suprimir o transporte. A competição entre esses dois efeitos "opostos" leva ao comportamento não-monotônico observado em $S_{\downarrow}$ como função de $e V$ [Fig. 4.17(d)].

Caso $p_{L} \neq p_{R}$. Na figura (4.18) mostramos gráficos para corrente e ruído no caso assimétrico $p_{L} \neq p_{R} \operatorname{com} p_{L}=0.23$ e $p_{R}=0.35$. A principal diferença dessa figura com relação ao caso $p_{L}=p_{R}$ está nas desigualdades $I_{\downarrow}>I_{\uparrow}$ e $S_{\downarrow}>S_{\uparrow}$ que ocorrem na configuração antiparalela e $U=0$. Note que para $p_{L}=p_{R}$ [Fig.(4.17)] temos $I_{\downarrow}=I_{\uparrow}$ e $S_{\downarrow}=S_{\uparrow}$ no alinhamento AP e $U=0$. Na presença de interação de Coulomb $(U=1 \mathrm{meV})$ a componente $\downarrow$ da corrente e do ruído é mais suprimida do que a componente $\uparrow$ devido ao bloqueio de Coulomb dependente de spin, conforme discutimos para a corrente na seção anterior.

Uma última observação é que, enquanto a corrente é nula para $\mathrm{eV} \rightarrow 0$, o ruído tem um valor diferente de zero nesse limite. Isso deve-se ao ruído térmico, que está presente mesmo em equilíbrio $(V=0)$. Em uma seção seguinte analisaremos melhor o ruído térmico.
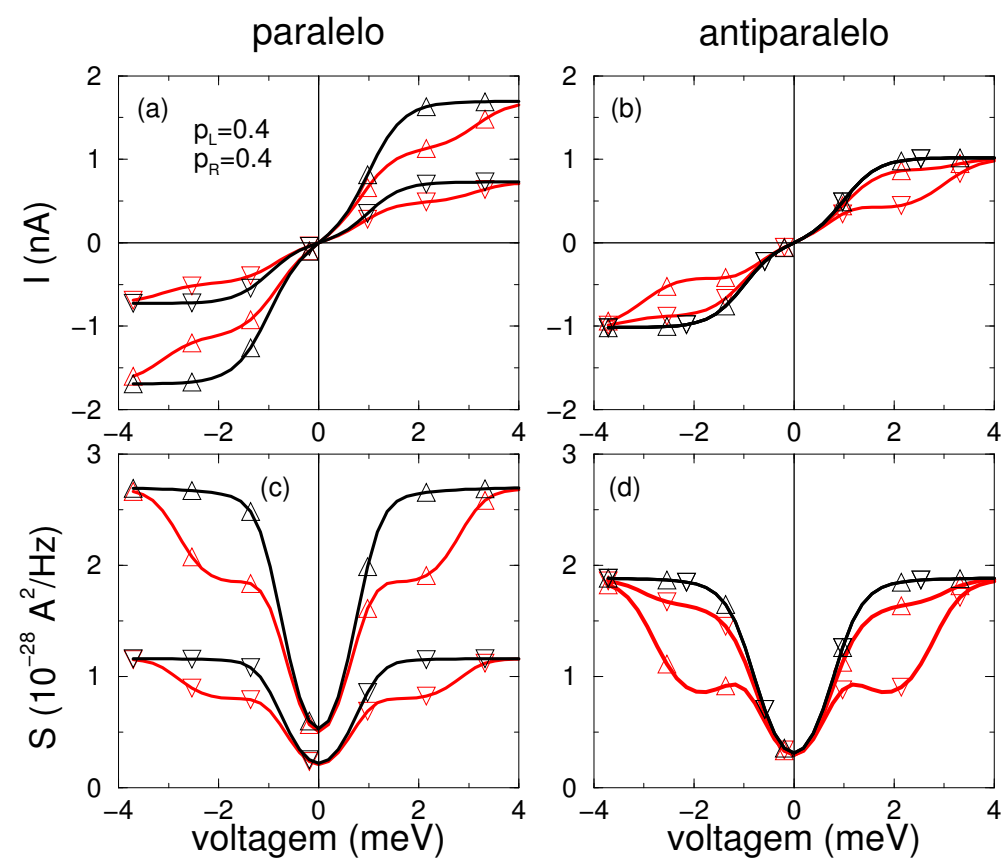

Figura 4.17: (a)-(b) Corrente e (c)-(d) ruído resolvidos em spin $\left(\triangle\right.$ para $I_{\uparrow}$ e $S_{\uparrow}$ e $\nabla$ para $I_{\downarrow}$ e $S_{\downarrow}$ ) como função da tensão $V, U=0$ (curvas pretas), $U=1 \mathrm{meV}$ (curvas vermelhas) e $p_{L}=p_{R}=0.4$. Os gráficos das correntes são idênticos aos da Fig.(4.11), e estão aqui apenas para efeitos de comparação com o ruído. Observamos que, assim como a corrente, o ruído também apresenta efeitos do bloqueio de Coulomb dependente de spin. Isso é evidente no painel (d) no qual temos para $U=0 S_{\uparrow}=S_{\downarrow}$ e para $U=1$ meV encontramos $S_{\uparrow} \neq S_{\downarrow}$, visto que uma das componentes de spin é mais fortemente suprimida. Parâmetros utilizados: $\Gamma_{0}^{L}=\Gamma_{0}^{R}=10 \mu \mathrm{eV}, \epsilon_{0}=0,5 \mathrm{meV}, k_{B} T=0.17 \mathrm{meV}$, $R=0$. 

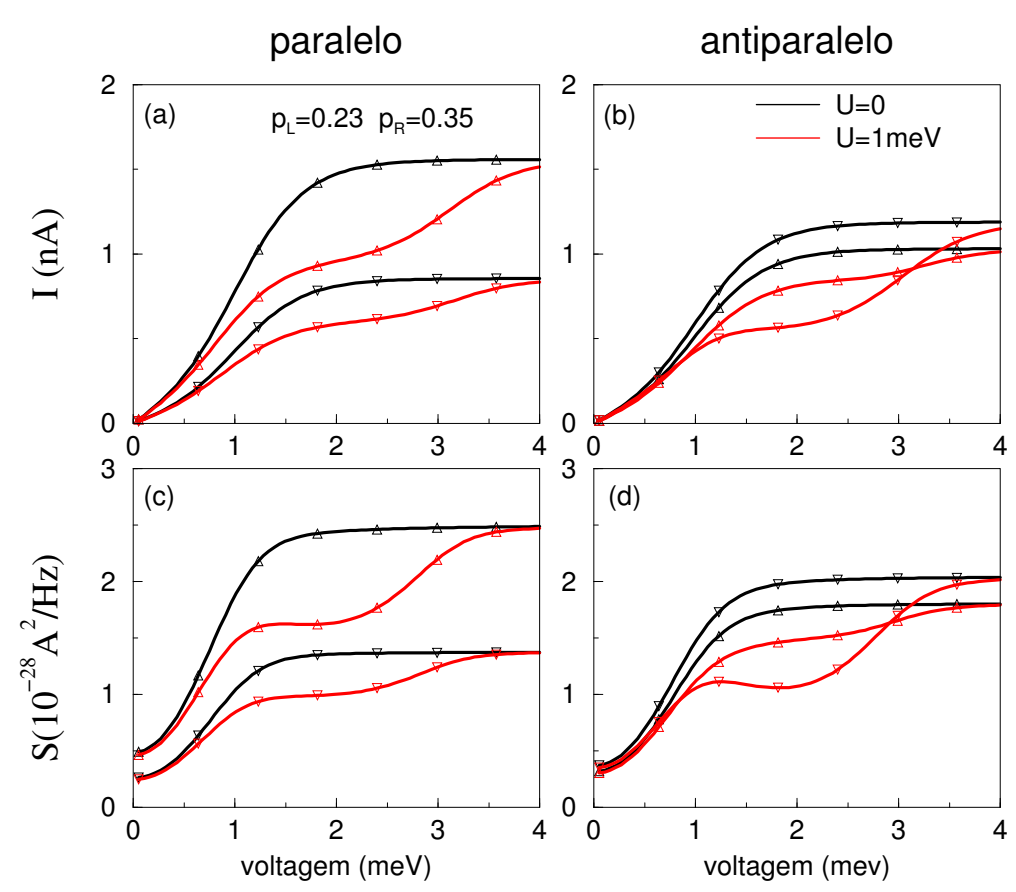

Figura 4.18: (a)-(b) Corrente e (c)-(d) ruído resolvidos em spin como função da tensão $V, U=0$ (curvas pretas), $U=1 \mathrm{meV}$ (curvas vermelhas), $p_{L}=0.23$, e $p_{R}=0.35$. A corrente e o ruído apresentam comportamentos parecidos. Por exemplo, no regime de bloqueio de Coulomb temos uma supressão do ruído assim como se dá com a corrente. Em particular, na configuração AP temos que $S_{\uparrow}<S_{\downarrow}$ para $U=0$ e o contrário, $S_{\uparrow}>S_{\downarrow}$, para $U=1 \mathrm{meV}$. A inversão dessa desigualdade, que também é observada na corrente [painel (b)], decorre do bloqueio de Coulomb dependente de spin. Parâmetros utilizados: $\Gamma_{0}^{L}=\Gamma_{0}^{R}=10 \mu \mathrm{eV}, \epsilon_{0}=0,5 \mathrm{meV}, k_{B} T=0.17 \mathrm{meV}, R=0$.

\subsubsection{Fator de supressão de ruído.}

Assim como para a corrente, podemos definir um parâmetro para quantificar a supressão do ruído no regime de bloqueio de Coulomb. Tomemos a variação $\Delta S_{\sigma}$ do ruído entre os casos $U=0$ e $U=1$ na região média do plateau das curvas $I-V$ e $S-V[e V \approx 2 \mathrm{meV}$ nas Figs.(4.17) e (4.18)], isto é,

$$
\begin{aligned}
\Delta S_{\uparrow} & =S_{\uparrow}^{U=0}-S_{\uparrow}^{U=1} \\
\Delta S_{\downarrow} & =S_{\downarrow}^{U=0}-S_{\downarrow}^{U=1} .
\end{aligned}
$$

Definindo o fator de supressão $\beta_{\sigma}$ de maneira análoga a definição de $\alpha_{\sigma}$ para a corrente,

$$
\begin{aligned}
& S_{\uparrow}^{U=1}=\frac{S_{\uparrow}^{U=0}}{\beta_{\uparrow}} \\
& S_{\downarrow}^{U=1}=\frac{S_{\downarrow}^{U=0}}{\beta_{\downarrow}},
\end{aligned}
$$


obtemos

$$
\begin{aligned}
& \Delta S_{\uparrow}=S_{\uparrow}^{U=0}-\frac{S_{\uparrow}^{U=0}}{\beta_{\uparrow}}=S_{\uparrow}^{U=0}\left(1-\frac{1}{\beta_{\uparrow}}\right) \\
& \Delta S_{\downarrow}=S_{\downarrow}^{U=0}-\frac{S_{\downarrow}^{U=0}}{\beta_{\downarrow}}=S_{\downarrow}^{U=0}\left(1-\frac{1}{\beta_{\downarrow}}\right) .
\end{aligned}
$$

Apliquemos agora essas equações para a configuração paralela e caso $p_{L}=p_{R}$. Extraímos da Fig.4.17(c) os seguintes valores no ponto médio do plateau ( $\mathrm{eV} \approx 2$ $\mathrm{meV}$ ): $S_{\uparrow}^{U=0}=2.64, S_{\downarrow}^{U=0}=1.15, S_{\uparrow}^{U=1}=1.86$ e $S_{\downarrow}^{U=1}=0.80$ (em unidades de $10^{-28}$ $\mathrm{A}^{2} / \mathrm{Hz}$ ). Com isso obtemos $\Delta S_{\uparrow}=0.78$ e $\Delta S_{\downarrow}=0.35$. Substituindo esses dados nas equações (4.33) e (4.34) vem

$$
\begin{aligned}
& \left(1-\frac{1}{\beta_{\uparrow}}\right)=\frac{\Delta S_{\uparrow}}{S_{\uparrow}^{U=0}}=0.30 \Rightarrow \beta_{\uparrow}=1.43 \\
& \left(1-\frac{1}{\beta_{\downarrow}}\right)=\frac{\Delta S_{\downarrow}}{S_{\downarrow}^{U=0}}=0.30 \Rightarrow \beta_{\downarrow}=1.43 .
\end{aligned}
$$

Portanto a supressão dos ruídos $S_{\uparrow}$ e $S_{\downarrow}$ no regime de bloqueio de Coulomb tem a mesma intensidade para ambas as componentes de spin na configuração paralela. Repetindo o mesmo cálculo para a configuração paralela do caso $p_{L} \neq p_{R}$ [Fig.4.18(c)] vem $S_{\uparrow}^{U=0}=2.44, S_{\downarrow}^{U=0}=1.35, S_{\uparrow}^{U=1}=1.64$ e $S_{\downarrow}^{U=1}=1.00$. Com isso temos $\Delta S_{\uparrow}=0.80$ e $\Delta S_{\downarrow}=0.35$ e portanto $\Delta S_{\uparrow} / S_{\uparrow}^{U=0}=0.33$ e $\Delta S_{\downarrow} / S_{\downarrow}^{U=0}=$ 0.26. Logo $\beta_{\uparrow}=1.50$ e $\beta_{\downarrow}=1.35$. Vemos então que para $p_{L} \neq p_{R}$ e configuração paralela, temos fatores de supressão diferentes para cada uma das componentes de spin, sendo maior para a componente $\uparrow$, assim como se dá com os fatores de supressão $\alpha_{\sigma}$ da corrente.

\subsubsection{Ruído térmico}

Na figura (4.19) ilustramos o ruído térmico $(e V=0)$ resolvido em spin como função da posição do nível $\epsilon_{d}$ do ponto quântico. Nessa figura temos $\mu_{L}=\mu_{R}$ pois $V=0$, e ambos se encontram no ponto zero (linha vertical verde). Observamos que, na configuração paralela, o ruído térmico é maior para spins $\uparrow(\triangle)$ do que para os spins $\downarrow(\nabla)$. Já na configuração antiparalela não há uma "polarização" do ruído térmico, isto é, as curvas para spins $\uparrow$ e $\downarrow$ coincidem. Esses resultados refletem a condutância $G$ do sistema, visto que o ruído térmico está relacionado a $G$ através da relação $S_{\sigma}=4 k_{B} T G_{\sigma}$ [64]. Logo, o que vemos na Fig.4.19(a) é simplesmente o fato de $G_{\uparrow}>G_{\downarrow}$ na configuração $\mathrm{P}$, e $G_{\uparrow}=G_{\downarrow}$ na configuração AP [Fig.4.19(b) ${ }^{4}$. O ruído térmico total $\left(S=S_{\uparrow}+S_{\downarrow}\right)$ também está ilustrado (linha tracejada). Observamos que ao passarmos da configuração P para AP, o ruído térmico $S$ tende a diminuir ligeiramente.

\footnotetext{
${ }^{4}$ Disso decorre a polarização da corrente $I_{\uparrow}>I_{\downarrow}$ no caso $\mathrm{P}$ e $I_{\uparrow}=I_{\downarrow}$ no caso AP, para $p_{L}=p_{R}=p$.
} 
Para entendermos o comportamento das curvas da Fig.(4.19), isto é, o fato de apresentarem um pico em torno de $\mu_{L}=\mu_{R}=0$, utilizamos a Fig.(4.20), onde mostramos a função espectral, cujo pico está centrado em $\epsilon_{d}$, e as funções de Fermi dos terminais $n_{L}$ e $n_{R}$. Nessa figura temos $n_{L}=n_{R}$ visto que $e V=0$. Para $\epsilon_{d} \approx \mu_{L}$ os elétrons dos reservatórios tem energia térmica suficiente para sofrerem transições dos terminais para o ponto-quântico e vice-versa, gerando assim um ruído térmico no sistema.

Quando $\epsilon_{d}=1 \mathrm{meV}>\mu_{L}$ o ruído térmico tende a zero, visto que não há energia térmica suficiente para excitação eletrônica entre eletrodos e ponto quântico. $\mathrm{O}$ ruído térmico também vai a zero para $\epsilon_{d}=-1 \mathrm{meV}<\mu_{L}$. Isso decorre da limitações no espaço de fase: os elétrons não são transferidos termicamente de um terminal a outro (via ponto quântico) pois os estados eletrônicos dos eletrodos estão todos preenchidos abaixo do nível de Fermi. Com isso temos então uma forma de se controlar a intensidade do ruído térmico através da tensão externa.

paralelo

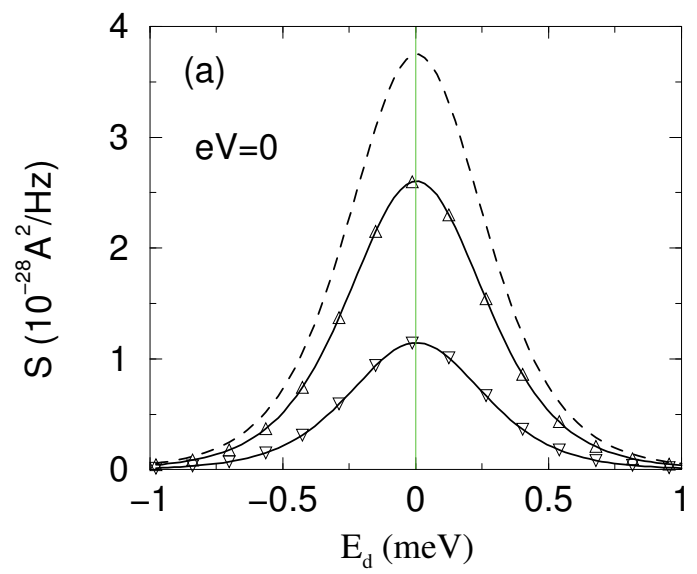

antiparalelo

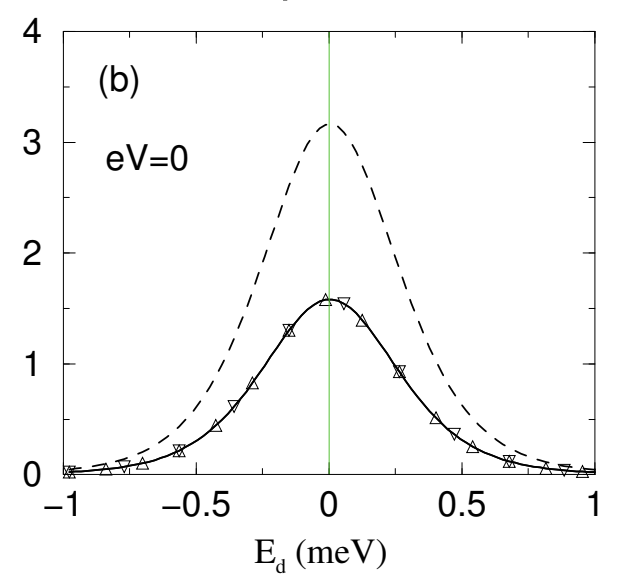

Figura 4.19: Ruido térmico como função da posição do nível $\epsilon_{d}$ nas configurações (a) paralela e (b) anti-paralela. Resolvemos em spin o ruído térmico, indicando spin $\uparrow$ por $\triangle$ e spin $\downarrow$ por $\nabla$. O ruído total $S_{\uparrow}+S_{\downarrow}$ está ilustrado através da linha tracejada. Os potenciais químicos $\mu_{L}$ e $\mu_{R}$ são iguais e estão posicionados em zero (linha vertical verde). Notamos que, ao passarmos da configuração $\mathrm{P}$ para AP, o ruído térmico total tende a diminuir. Também observamos uma "polarização" do ruído térmico no alinhamento paralelo $\left(S_{\uparrow}>S_{\downarrow}\right)$, ao contrário do caso AP em que $S_{\uparrow}=S_{\downarrow}$. Observamos que para $\epsilon_{d} \approx 0$ temos um ruído térmico máximo em ambos os alinhamentos magnéticos. Isso ocorre porque nesse caso, os elétrons dos terminais tem energia térmica suficiente para tunelar ressonantemente pelo ponto quântico. Para $\epsilon_{d}=1 \mathrm{meV}$ a energia térmica é insuficiente para ocorrer esse tunelamento, resultando num ruído térmico nulo tanto em (a) quanto em (b). Para $\epsilon_{d}=-1 \mathrm{meV}$, apesar dos elétrons terem energia térmica suficiente, os estados eletrônicos dos terminais estão todos ocupados. Logo não há espaço de fase disponível para haver transições de um terminal a outro (via ponto quântico). Outros parâmetros: $U=0, R=0, k_{B} T=0.17 \mathrm{meV}, p_{L}=p_{R}=0.4$. 


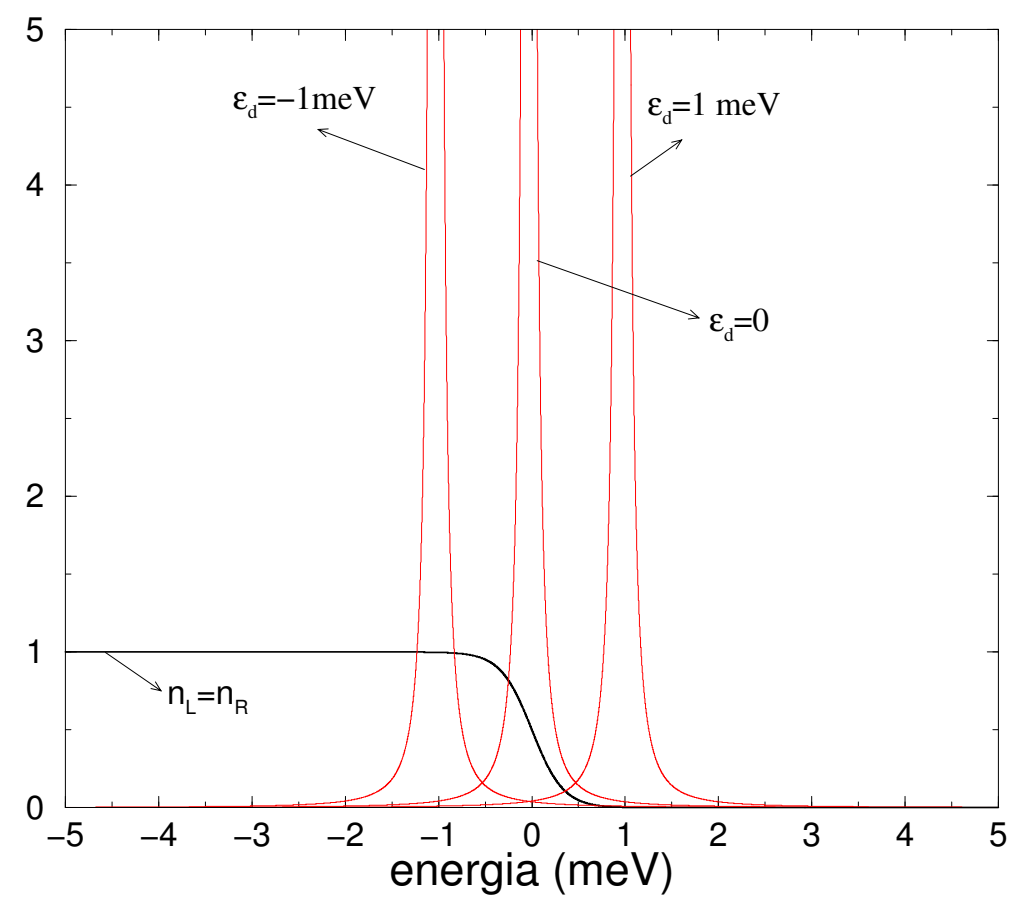

Figura 4.20: Função espectral (linhas vermelhas) para três valores de $\epsilon_{d}$. As funções de Fermi $n_{L}$ e $n_{R}$ também estão graficadas $\left(n_{L}=n_{R}\right.$ pois $\left.V=0\right)$. Para $\epsilon_{d}=1 \mathrm{meV}$ temos que o nível do ponto quântico (linha vermelha) está acima de $\mu_{L}$, logo os elétrons dos terminais não tem energia térmica suficiente para tunelar ressonantemente. Isso resulta num ruído térmico nulo. Para $\epsilon_{d}=-1 \mathrm{meV}$ o ruído térmico também vale zero, pois como $\epsilon_{d}$ está abaixo de $\mu_{L}$ e de $\mu_{R}$, não há estados eletrônicos acessíveis para que haja fluxo aleatório de elétrons entre os terminais. Para $\epsilon_{d}=0$ pode have transições de elétrons de um terminal a outro, pois um elétron de um terminal pode atravessar o ponto quântico e ocupar um estado vazio do terminal oposto. 
Efeitos do espalhamento de spin sobre o ruído térmico. Na figura (4.21) ilustramos o ruído térmico $(e V=0)$ como função da posição do nível $\epsilon_{d}$, para diferentes valores do parâmetro de espalhamento de spin $R$. Encontramos que o ruído térmico na configuração anti-paralela aumenta à media em que $R$ cresce (adota$\operatorname{mos} R=0, R=6 \mu \mathrm{eV}$ e $R=30 \mu \mathrm{eV}$ ). Por outro lado, o ruído no caso paralelo é insensível a $R$ (linha azul). Para $R$ suficientemente grande temos que o ruído térmico nas duas configurações magnéticas se igualam. Vale lembrar que um efeito análogo foi observado para as correntes $I_{P}$ e $I_{A P}$, onde $I_{A P}$ convergia para $I_{P}$ com o aumento de $R$ [veja Fig.(4.13)]. A diferença é que aqui estamos tratando de um sinal de equilíbrio (ruído térmico a $\mathrm{eV}=0$ ).

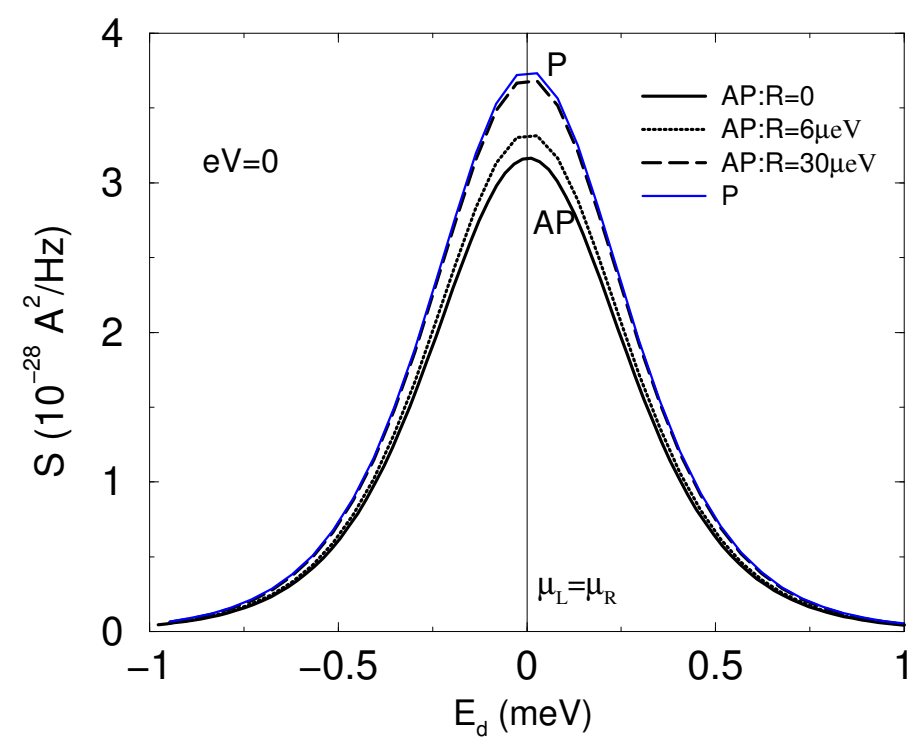

Figura 4.21: Ruído térmico como função da posição do nível $\epsilon_{d}$ para diferentes valores do parâmetro de espalhamento de spin $R$. Observamos que o ruído no alinhamento AP converge para o seu valor no caso $\mathrm{P}$ a medida em que $R$ aumenta.

\subsubsection{Shot noise na presença de espalhamento de spin}

Vejamos agora os efeitos do espalhamento de spin sobre o ruído. Na figura (4.22) mostramos o ruído como função da tensão externa para $U=0, U=1 \mathrm{meV}$, e diferentes parâmetros de espalhamento de spin $R$. Também mostramos curvas para a condutância diferencial $G=d I / d V$ multiplicada por $4 k_{B} T$, isto é, $4 k_{B} T G$. Observamos que, no caso paralelo [painel (a)], o ruído é insensível ao parâmetro $R$, semelhantemente à corrente [Fig.4.13(a)]. A condutância $G$ também não depende de $R$ no alinhamento paralelo. Já na configuração AP o ruído varia com $R$. Observe que o shot noise [veja limite $\mathrm{eV} \rightarrow 5 \mathrm{meV}$ ] tem um comportamento não-monotônico com $R$. Seu valor inicialmente diminui e em seguida aumenta, apresentando assim um ponto de mínimo para um determinado $R$. Esse comportamento não monotônico será analisado em maiores detalhes na próxima figura. 
paralelo

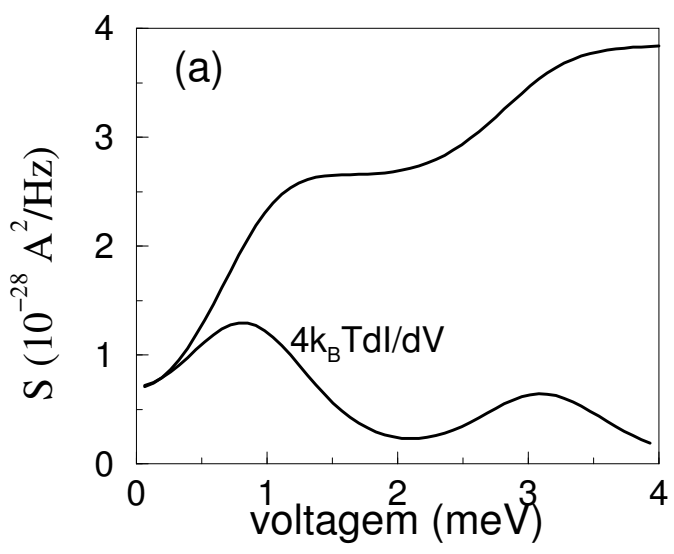

antiparalelo

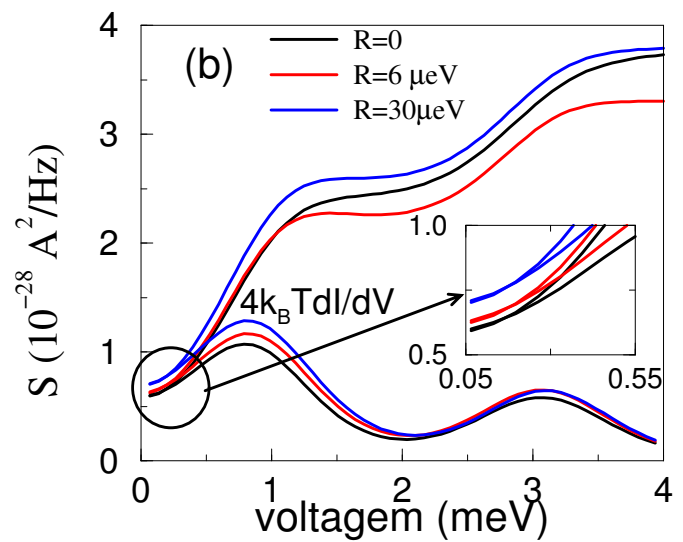

Figura 4.22: Ruído total $\left(S_{\uparrow}+S_{\downarrow}\right)$ versus tensão nos alinhamentos (a) paralelo e (b) antiparalelo. O ruído no caso $\mathrm{P}$ é insensível a $R$. Já na configuração AP temos uma variação não monotônica de $S$, que é inicialmente suprimido para $R=6 \mu \mathrm{eV}$ e em seguida aumenta para $R=30 \mu \mathrm{eV}$. Curvas para a função $4 k_{B} T d I / d V$ também estão apresentadas. Note que para $e V \rightarrow 0$ (resposta linear) o ruído $S$ tende a $4 k_{B} T d I / d V$. No inset do painel (b) fazemos uma ampliação das curvas na região próxima da origem, onde podemos perceber que $S \rightarrow 4 k_{B} T d I / d V$ mesmo na presença de espalhamento de spin. Outros parâmetros $U=1 \mathrm{meV}, k_{B} T=0.17 \mathrm{meV}, \epsilon_{0}=0.5 \mathrm{meV}$.

Com relação à condutância $G$, os dois pontos de máximo correspondem aos canais de condução $\epsilon_{1}$ e $\epsilon_{2}$ (primeiro pico), $\epsilon_{1}+U$ e $\epsilon_{2}+U$ (segundo pico) ${ }^{5}$. No limite de tensão nula verificamos que $S=4 k_{B} T G$, conforme o esperado [64] $]^{6}$. Note que $G$ não depende de $R$ na configuração paralela, mas varia ligeiramente no caso AP.

A dependência não-monotônica do ruído na configuração AP está melhor ilustrada na figura (4.23), onde mostramos a corrente [painel (a)], o ruído $S$ [painel (b)] e o fator de Fano $\gamma=S /(2 e I)$ [painel (c)] contra $R$ para diferentes valores do parâmetro de polarização $p_{L}=p_{R}=p$. Os casos $U=0$ e $U=1 \mathrm{meV}$ foram graficados (linhas sólidas e tracejadas, respectivamente). A tensão adotada nessa figura foi de $\mathrm{eV}=4 \mathrm{meV}$, e portanto estamos acima do bloqueio de Coulomb no presente gráfico.

A corrente $I_{A P}$ cresce monotonicamente com $R$, tendendo para o seu valor na configuração paralela $I_{P}$. O ruído, por outro lado, tem um comportamento nãomonotônico com $R$, apresentando um mínimo para um dado valor de $R$. Para $R$ suficientemente grande, temos que $S_{A P}$ (ruído na configuração AP) tende para $S_{P}$ (ruído na configuração $\mathrm{P}$ ). Ressaltamos que $S_{P}$ é insensível a $R$, assim como a corrente $I_{P}$. A presença de interação de Coulomb no sistema faz com que as

\footnotetext{
${ }^{5}$ Os picos $\epsilon_{1}$ e $\epsilon_{1}$ estão tão próximos ( $R$ pequeno) que não são distinguidos pela condutância.

${ }^{6}$ Vale salientar que corrente e ruído são calculados de maneira distintas, assim a verificação da relação $S=4 k_{B} T d I / d V$ em baixas tensões é uma forma de testar os resultados numéricos.
} 
curvas de $I_{A P}$ subam mais rápido como função de $R$, indicando que a interação de Coulomb aumenta a "eficácia" do espalhamento de spin. Do mesmo modo, $S_{A P}$ atinge seu mínimo primeiro do que no caso $U=0$ e em seguida sobe mais rápido. Esse comportamento também indica que o efeito combinado da interação de Coulomb e do espalhamento de spin leva a uma intensificação do mecanismo de espalhamento de spin. 

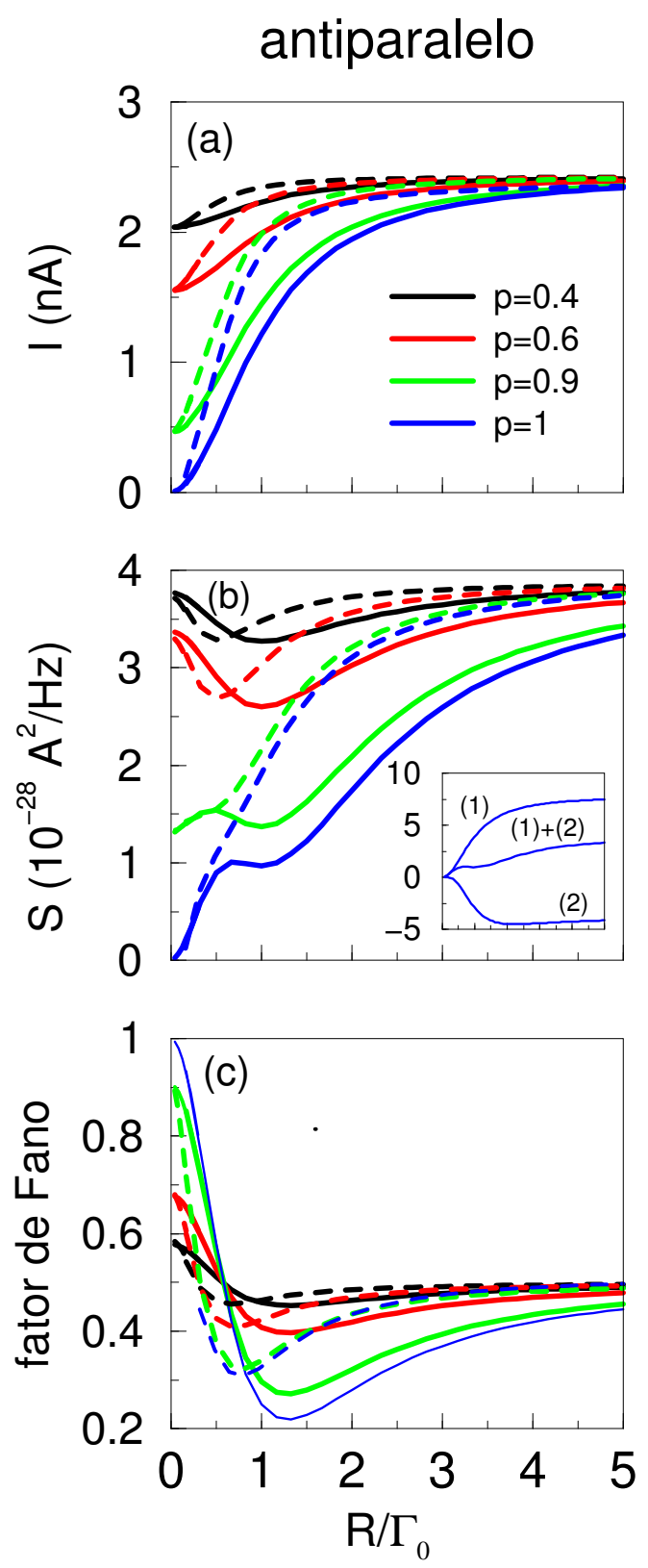

Figura 4.23: (a) Corrente, (b) ruído e (c) fator de Fano como função de $R$, para diferentes parâmetros de polarização $p_{L}$ e $p_{R}\left(p_{L}=p_{R}=p\right), U=0$ (linha sólida) e $U=1 \mathrm{meV}$ (linha tracejada). Enquanto a corrente cresce monotonicamente com $R$, o ruído apresenta um mínimo. Essa supressão do ruído também é observada no fator de Fano [painel (c)], que atinge valores inferiores a 0.5. Isso indica que o espalhamento de spin introduz correlações adicionais no sistema que resultam em $\gamma<0.5$. Observamos ainda que essas correlações intensificam-se a medida que a polarização dos eletrodos aumenta, pois quanto maior $p$, menor são os valores atingidos por $\gamma$. No inset mostramos as soluções analíticas para o ruído no caso $p=1$, que coincide com o resultado numérico do painel (b). Também apresentamos os dois termos analíticos $\left[(1) \propto \int T\right]$ e $\left[(2) \propto \int T^{2}\right]$ separadamente. 


\subsubsection{Fator de Fano}

Uma outra forma de analisar a dependência do ruído como função de $R$ é através do fator de Fano $\gamma=S /(2 e I)$. Salientamos que $\gamma$ mede o quão correlacionado é a transmissão de elétrons pelo sistema. Por exemplo, para $\gamma=1$ temos $S=2 e I$, que é o valor clássico (descorrelacionado) do ruído. Quando $\gamma<1$ há algum tipo de correlação, como Pauli, Coulomb, que pode levar a um atraso na transmissão de elétrons, e conseqüentemente suprimir o ruído.

Observamos que o espalhamento de spin leva a uma supressão do fator de Fano. Lembremos que, para um sistema de dupla-barreira com tunelamento ressonante e sem interações, espera-se uma supressão de $\gamma=0.5$ para barreiras simétricas, e $0.5<\gamma<1$ para barreiras assimétricas. Portanto, a supressão adicional observada na Fig.4.23(c) deve-se a correlações adicionais no sistema provenientes do espalhamento de spin. Conforme veremos em seções subseqüentes, essa correlação adicional nada mais é do que uma intensificação da correlação gerada pelo princípio de Pauli. Em particular, para $p=1$ e configuração AP obtemos uma supressão máxima em torno de $\gamma \approx 0.2$. Na configuração paralela, o espalhamento de spin não produz nenhum efeito sobre o fator de Fano, que permanece em torno de 0.5.

\subsubsection{Expressão analítica para o ruído}

Podemos obter expressões analíticas para o ruído e o fator de Fano no caso AP que nos ajudem a entender a supressão adicional do ruído observada na Fig.4.23(c). Assumimos $p_{L}=p_{R}=1$ (caso $100 \%$ polarizado), $U=0$ e tensão grande o suficiente para que os níveis $\epsilon_{1}$ e $\epsilon_{2}$ estejam dentro da "janela" de condução: intervalo entre $\mu_{L}$ e $\mu_{R}$. Com esses parâmetros podemos obter uma expressão analítica para o ruído a partir da Eq.(3.43). Como em tensão suficientemente grande podemos fazer $n_{L}=1$ e $1-n_{L}=0$, a Eq.(3.43) fica

$$
S=\frac{2 e^{2}}{\hbar} \int \frac{d \epsilon}{2 \pi} \operatorname{Tr}\left\{i \boldsymbol{\Gamma}^{L} \mathbf{G}^{>}+\left(\mathbf{G}^{r}-\mathbf{G}^{a}\right) \boldsymbol{\Gamma}^{L} \mathbf{G}^{>} \boldsymbol{\Gamma}^{L}+\mathbf{G}^{<} \boldsymbol{\Gamma}^{L} \mathbf{G}^{>} \boldsymbol{\Gamma}^{L}\right\} .
$$

Usando agora a igualdade $\mathbf{G}^{>}=\mathbf{G}^{r}-\mathbf{G}^{a}+\mathbf{G}^{<}$na equação (4.36) vem

$$
\begin{aligned}
S=\frac{2 e^{2}}{\hbar} \int \frac{d \epsilon}{2 \pi} & \operatorname{Tr}\left\{i \boldsymbol{\Gamma}^{L}\left(\mathbf{G}^{r}-\mathbf{G}^{a}\right)+i \boldsymbol{\Gamma}^{L} \mathbf{G}^{<}+\left(\mathbf{G}^{r}-\mathbf{G}^{a}\right) \boldsymbol{\Gamma}^{L}\left(\mathbf{G}^{r}-\mathbf{G}^{a}\right) \boldsymbol{\Gamma}^{L}\right. \\
& \left.+\left(\mathbf{G}^{r}-\mathbf{G}^{a}\right) \boldsymbol{\Gamma}^{L} \mathbf{G}^{<} \boldsymbol{\Gamma}^{L}+\boldsymbol{\Gamma}^{L} \mathbf{G}^{<} \boldsymbol{\Gamma}^{L}\left(\mathbf{G}^{r}-\mathbf{G}^{a}\right)+\boldsymbol{\Gamma}^{L} \mathbf{G}^{<} \boldsymbol{\Gamma}^{L} \mathbf{G}^{<}\right\},
\end{aligned}
$$

que pode ser escrita assim

$$
\begin{aligned}
S=\frac{2 e^{2}}{\hbar} \int \frac{d \epsilon}{2 \pi} \operatorname{Tr}\left(i \boldsymbol{\Gamma}^{L}\left(\mathbf{G}^{r}-\mathbf{G}^{a}\right)+i \boldsymbol{\Gamma}^{L} \mathbf{G}^{<}\right) \times \\
\left(1-i \boldsymbol{\Gamma}^{L}\left(\mathbf{G}^{r}-\mathbf{G}^{a}\right)-i \boldsymbol{\Gamma}^{L} \mathbf{G}^{<}\right) .
\end{aligned}
$$

Definindo $\tau=\Gamma^{L}\left(\mathbf{G}^{r}-\mathbf{G}^{a}\right)+\Gamma^{L} \mathbf{G}^{<}$vem

$$
S=\frac{2 e^{2}}{\hbar} \int \frac{d \epsilon}{2 \pi} \operatorname{Tr}\{i \tau(1-i \tau)\}
$$


e definindo $\mathbf{T}=i \tau$ encontramos

$$
S=\frac{2 e^{2}}{\hbar} \int \frac{d \epsilon}{2 \pi} \operatorname{Tr}\{\mathbf{T}(1-\mathbf{T})\}
$$

que tem a forma geral da expressão de Landauer-Büttiker encontrada na literatura [64]. Porém, ressaltamos que a nossa Eq.(4.39) leva em conta espalhamento de spin.

No inset da Fig.4.23(b) graficamos os termos $\int \operatorname{Tr} \mathbf{T}$ e $\left(-\int \operatorname{Tr} \mathbf{T}^{2}\right)$ separadamente [linhas (1) e (2), respectivamente] e a Eq.(4.39) [linha (1)+(2)]. O primeiro termo cresce monotonicamente com $R$ (semelhante a corrente), enquanto o segundo decresce. O decaimento do termo (2) dá origem a supressão do ruído. Portanto, vemos que essa supressão, gerada pelo espalhamento de spin surge através do termo quadrático no coeficiente de transmissão.

Fator de Fano. Aqui apresentamos uma fórmula para o fator de Fano que nos permite fazer uma analogia entre o nosso sistema e um problema de tripla-barreira. Essa analogia nos ajuda a entender fisicamente a origem da supressão adicional da ruído, provocada pelo espalhamento de spin.

Assumindo $p_{L}=p_{R}=1$ (portanto terminais $100 \%$ polarizados), configuração anti-paralela e $U=0$, temos para a corrente a seguinte equação

$$
I=\frac{e}{\hbar} \int \frac{d \epsilon}{2 \pi} T(\epsilon)
$$

onde $T$ está definido na Eq.(4.24), e sendo o ruído

$$
S=\frac{2 e^{2}}{\hbar} \int \frac{d \epsilon}{2 \pi} T(\epsilon)[1-T(\epsilon)]
$$

obtemos

$$
\gamma=\frac{S}{2 e I}=1-\frac{\int d \epsilon T(\epsilon) T(\epsilon)}{\int d \epsilon T(\epsilon)} .
$$

A equação acima pode ser resolvida analiticamente, o que nos dá

$$
\gamma=1-\frac{1}{2} \frac{\beta^{2}\left(5+\beta^{2}\right)}{\left(1+\beta^{2}\right)^{2}}
$$

onde $\beta=R / \Gamma_{0}$. Ressaltamos que o gráfico dessa equação, como função de $R$, coincide com a curva em azul ( $p=1)$ da Fig. 4.23(c), que foi obtida numericamente.

Curiosamente, podemos mostrar que, para um sistema de três barreiras com um único nível ressonante em cada poço [veja Fig.(4.24)] o fator de Fano é dado também pela Eq.(4.43) 7 . Isso mostra que há uma analogia entre o sistema FMPQ-FM na configuração AP $100 \%$ polarizada, $U=0$ e $R \neq 0$, com o sistema de três barreiras ilustrado na Fig.(4.24). Nessa analogia o espalhamento de spin corresponde a um processo de tunelamento através da barreira intermediária [indicada por $R$ na Fig.(4.24)]. O estado localizado na região I da Fig.(4.24) equivale a

\footnotetext{
${ }^{7}$ Utilizando alguns dos resultados da referência [85], obtidos para um sistema de três barreiras com um único nível ressonante em cada poço, podemos obter a nossa Eq.(4.43).
} 
$\epsilon_{\uparrow}$ e na região II temos $\epsilon_{\downarrow}$. Assim, para um elétron passar de $\epsilon_{\uparrow}$ para $\epsilon_{\downarrow}$ é necessário tunelar a barreira intermediária, ou equivalentemente espalhar o seu spin. Quanto maior $R$, menor é a barreira do meio, de modo que os estados $\uparrow$ e $\downarrow$ tendem a se acoplar mais. Note que a barreira de entrada (L) é dada por $\Gamma_{\uparrow}^{L}$ e a de saída por $\Gamma_{\downarrow}^{R}$. Isso deve-se a hipótese de terminais $100 \%$ polarizados (a esquerda temos polarização $\uparrow$ e a direita $\downarrow$ ). Com essa analogia, podemos entender a supressão do ruído gerada pelo espalhamento de spin, conforme discutimos no próximo parágrafo.

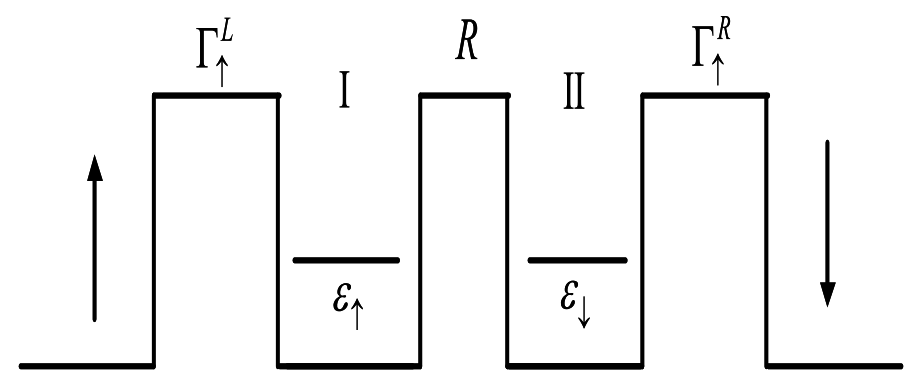

Figura 4.24: Sistema de três barreiras análogo ao sistema FM-PQ-FM no caso AP 100\% polarizado com espalhamento de spin. Nessa analogia o espalhamento de spin equivale a um processo de tunelamento através da barreira central (indicada por $R$ ).

Lembremos que, num problema usual de dupla-barreira com um nível ressonante, um elétron que sai do emissor e entra na região central (por exemplo o ponto quântico) permanece aí por um certo tempo dado pelas taxas $\Gamma_{\sigma}^{\eta}$ de entrada e de saída. Durante esse intervalo, a entrada de um segundo elétron de mesmo spin fica bloqueada pelo princípio de Pauli. Vemos então que o princípio de Pauli correlaciona o fluxo de elétrons. No caso de barreiras simétricas, essa correlação resulta em $\gamma=0.5$, e para barreiras assimétricas temos $\gamma \rightarrow 1$ a medida que a assimetria aumenta [46].

Ao contrário do sistema de duas barreiras, onde o elétron ao sair da região central indo para o terminal coletor, não retorna mais à região central, no sistema de três barreiras um elétron que sofre a transição I $\rightarrow$ II (sai da região I e vai para II) pode voltar a região I através da transição reversa II $\rightarrow$ I. Com isso o bloqueio de Pauli intensifica-se, o que leva a uma maior correlação no fluxo de portadores e conseqüentemente a uma supressão mais intensa do ruído.

Nessa visão de tripla-barreira, podemos ainda entender o fato da supressão do ruído aumentar com a polarização dos terminais [veja Fig.4.23(c)]. Sabemos que um elétron de spin $\uparrow$ bloqueia a entrada de um segundo elétron de mesmo spin, 
mas não bloqueia a entrada de um spin $\downarrow$ (não estamos considerando bloqueio de Coulomb $)^{8}$. Assim, se só houver spins $\uparrow$ no emissor $(p=1)$ o bloqueio de Pauli será máximo. Ao contrário, se $p<1$ um elétron de spin $\downarrow$ poderá entrar no ponto quântico, resultando num bloqueio de Pauli menos eficiente, e conseqüentemente numa supressão menor.

Por último, analisamos os efeitos da assimetria das barreiras de entrada e de saída do ponto quântico sobre o ruído. Sabemos que $\gamma \rightarrow 1$ em sistemas de dupla barreira assimétricas. Porém, na presença de espalhamento de spin, a supressão do ruído pode resultar em $\gamma<1$ mesmo para sistemas extremamente assimétricos. A figura (4.25) mostra o fator de Fano para a configuração AP 100\% polarizada. Os parâmetros utilizados são tais que $\Gamma_{\downarrow}^{R}$ é (a) muito maior do que $\Gamma_{\uparrow}^{L}$ e (b) igual a $\Gamma_{\uparrow}^{L}$. Desse modo temos um caso extremamente assimétrico e outro simétrico, respectivamente. Para $R$ grande o suficiente a barreira intermediária da Fig.(4.24) tende a zero e o sistema é governado pelas barreiras de entrada e de saída. Isso explica os limites assintóticos das curvas. No caso (a) o fator de Fano tende a 1 quando $R$ aumenta. Isso ocorre devido a forte assimetria do sistema. Para o caso (b) obtemos 0.5 no limite de $R$ grande. Esse valor decorre da simetria entre as barreiras de entrada e de saída. Note que, no caso extremamente assimétrico apesar de esperarmos $\gamma \approx 1$, podemos obter $\gamma \approx 0.5$ devido a espalhamento de spin.

Com esse capítulo procuramos abordar alguns aspectos da corrente e do ruído no sistema FM-PQ-FM, na presença de espalhamento de spin e interação de Coulomb. No capítulo seguinte, analisaremos a corrente spin polarizada em um sistema de ponto quântico acoplado a multiplos terminais ferromagnéticos, ao invés de somente dois terminais como aqui. Vale antecipar que o fato de se ter mais de dois terminais ferromagnéticos leva a efeitos interessantes, como a amplificação da polarização da corrente que sai de um terminal emissor e simultaneamente a inversão do seu sinal.

\footnotetext{
${ }^{8} \mathrm{Na}$ presença de interação de Coulomb, além do bloqueio de Pauli, surge o bloqueio de Coulomb.
} 


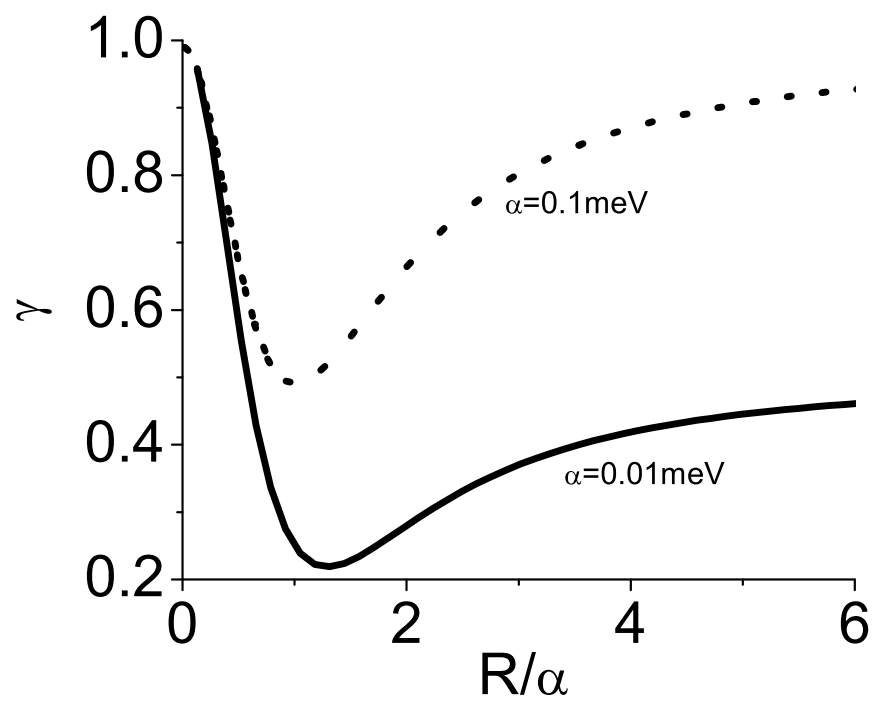

Figura 4.25: Fator de Fano como função de $R$ para a configuração AP $100 \%$ polarizada $\left(p_{L}=p_{R}=1\right)$ e $U=0$. As curvas sólida e tracejada correspondem a $\Gamma_{0}^{R}=\Gamma_{0}=10 \mu \mathrm{eV}$ e $\Gamma_{0}^{R}=100 \Gamma_{0}$, respectivamente. Em ambos os casos temos $\Gamma_{0}^{L}=\Gamma_{0}$. Logo, temos um caso simétrico (linha sólida) e outro extremamente assimétrico (linha tracejada). No caso assimétrico o fator de Fano inicia em 1, em seguida é suprimido para valores em torno de 0.5 , e retorna a 1 para $R$ 's grandes. Vemos então que, para um sistema fortemente assimétrico, o fator de Fano pode ser da ordem de 0.5 se o espalhamento de spin estiver presente. Nos limites assintóticos dessas curvas temos $\gamma=0.5$ (linha sólida) e $\gamma=1$ (linha tracejada), o que corresponde aos casos simétricos e assimétricos, respectivamente. Portanto para $R$ grandes o ruído é governado pelas barreiras de entrada e de saída do ponto quântico. 


\section{Capítulo 5}

\section{Gerador simultâneo de correntes com polarizações $\uparrow \mathbf{e} \downarrow$}

Nesse capítulo estudamos um sistema constituído por um ponto quântico acoplado, via barreiras de tunelamento, a multiplos terminais ferromagnéticos. Em particular para o caso de três terminais, mostramos que esse sistema pode amplificar a polarização da corrente em um determinado terminal, e simultaneamente mudar o sinal da polarização em um outro terminal. Portanto, temos dois efeitos combinados e simultâneos: (i) amplificação da polarização da corrente e (ii) inversão de sua polarização.

\section{$5.1 \quad$ Modelo}

O sistema estudado no presente capítulo é composto de um ponto quântico acoplado, via barreiras de tunelamento, a vários terminais ferromagnéticos, conforme ilustra a figura (5.1). Cada terminal tem um potencial químico $\mu_{j}$, que pode variar segundo uma tensão externa. As várias correntes de tunelamento indicadas pelas setas na figura (5.1), dependem das diferenças entre esses potenciais químicos $\mu_{j}$.

Para descrever o sistema de um ponto quântico acoplado a multiplos terminais, utilizamos o seguinte Hamiltoniano

$$
H=\sum_{k \sigma j} \epsilon_{k \sigma j} c_{k \sigma j}^{\dagger} c_{k \sigma j}+\sum_{\sigma} \epsilon_{\sigma} d_{\sigma}^{\dagger} d_{\sigma}+\sum_{k \sigma j}\left(t_{k \sigma j} c_{k \sigma j}^{\dagger} d_{\sigma}+t_{k \sigma j}^{*} d_{\sigma}^{\dagger} c_{k \sigma j}\right),
$$

onde $\epsilon_{k \sigma j}$ é a energia de um elétron no terminal $j$, com vetor de onda $k$ e spin $\sigma$, sendo $c_{k \sigma j}\left(c_{k \sigma j}^{\dagger}\right)$ o operador destruição (criação) de elétrons no contato de índice $j$. $\epsilon_{\sigma}$ é o nível de energia no ponto quântico, $d_{\sigma}\left(d_{\sigma}^{\dagger}\right)$ o operador destruição (criação) de elétrons no ponto quântico, e $t_{k \sigma j}$ o elemento de matriz que acopla o ponto quântico ao j-ésimo contato. No presente modelo não incluímos interação de Coulomb nem espalhamento de spin, e consideramos o nível do ponto quântico degenerado $\epsilon_{\uparrow}=\epsilon_{\downarrow}=\epsilon_{d}$. A variação desse nível em função da tensão externa é $\epsilon_{d}=\epsilon_{0}-e V / 2$, onde $\epsilon_{0}$ é a posição do nível sem tensão externa aplicada. 
Posteriormente vamos aplicar nossos resultados para o caso particular de três terminais ferromagnéticos acoplados ao ponto quântico. Porém, vamos desenvolver o formalismo para um número arbitrário de terminais de modo a se obter uma expressão geral para a corrente.

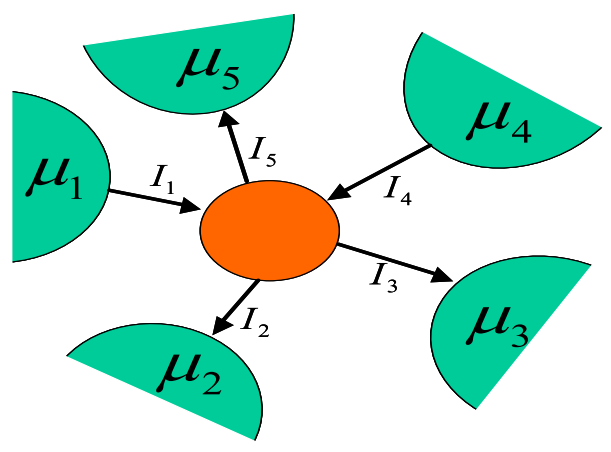

Figura 5.1: Sistema composto de vários terminais ferromagnéticos acoplados a um ponto quântico. Cada terminal tem um potencial químico $\mu_{j}(j=1,2, \ldots)$ que pode variar segundo uma tensão externa aplicada. Essas tensões estabelecem diferenças de potencial entre os vários terminais, gerando correntes de tunelamento $I_{j}$.

\subsection{Corrente}

Para calcularmos a corrente vamos partir da definição $I_{j}=-e\left\langle\dot{N}_{j}\right\rangle$, onde $\dot{N}_{j}=$ $\frac{i}{\hbar}\left[H, N_{j}\right]$, sendo $N_{j}$ o operador correspondente ao número total de elétrons no $j$-ésimo terminal $\left(N_{j}=\sum_{k \sigma} c_{k \sigma j}^{\dagger} c_{k \sigma j}\right)$. Utilizando o Hamiltoniano dado em (5.1) calculamos o comutador $\left[H, N_{j}\right]$ e encontramos para a corrente no j-ésimo terminal

$$
I_{j}=\frac{i e}{\hbar} \sum_{k \sigma}\left\{t_{k \sigma j}\left\langle c_{k \sigma j}^{\dagger} d_{\sigma}\right\rangle-t_{k \sigma j}^{*}\left\langle d_{\sigma}^{\dagger} c_{k \sigma j}\right\rangle\right\}
$$

que pode ser reescrito na forma

$$
I_{j}=-\frac{2 e}{\hbar} \operatorname{Re} \sum_{k \sigma} t_{k \sigma j}^{*} G_{k \sigma j, \sigma}^{<}(t, t),
$$

onde definimos a função de Green menor $G_{k \sigma j, \sigma}^{<}\left(t, t^{\prime}\right)=i\left\langle d_{\sigma}^{\dagger}\left(t^{\prime}\right) c_{k \sigma j}(t)\right\rangle$, em analogia a equação (3.13) do capítulo 3. Conforme discutimos na seção 3.3 e no apêndice A, para obtermos $G_{k \sigma j, \sigma}^{<}\left(t, t^{\prime}\right)$ adotamos os seguintes passos: calculamos uma equação 
para a função de Green ordenada no contorno $G_{k \sigma j, \sigma}\left(\tau, \tau^{\prime}\right)=-i\left\langle T_{c} c_{k \sigma j}(\tau) d_{\sigma}^{\dagger}\left(\tau^{\prime}\right)\right\rangle$, e em seguida efetuamos a continuação analítica (Cap.2) para encontrar $G_{k \sigma j, \sigma}^{<}\left(t, t^{\prime}\right)$. O resultado obtido é análogo à Eq.(3.14), e está dado abaixo

$$
G_{k \sigma j, \sigma}^{<}\left(t, t^{\prime}\right)=t_{k \sigma j} \int d t_{1}\left[g_{k \sigma j}^{r}\left(t, t_{1}\right) G_{\sigma \sigma}^{<}\left(t_{1}, t^{\prime}\right)+g_{k \sigma j}^{<}\left(t, t_{1}\right) G_{\sigma \sigma}^{a}\left(t_{1}, t^{\prime}\right)\right] .
$$

Substituindo esse resultado em (5.3) obtemos

$$
\begin{gathered}
I_{j}=-\frac{2 e}{\hbar} \operatorname{Re} \sum_{k \sigma}\left|t_{k \sigma j}\right|^{2} \int d t_{1}\left[g_{k \sigma j}^{r}\left(t, t_{1}\right) G_{\sigma \sigma}^{<}\left(t_{1}, t^{\prime}\right)+g_{k \sigma j}^{<}\left(t, t_{1}\right) G_{\sigma \sigma}^{a}\left(t_{1}, t^{\prime}\right)\right] \\
=-\frac{e}{\hbar} \sum_{k \sigma}\left|t_{k \sigma j}\right|^{2} \int d t_{1}\left\{\left[g_{k \sigma j}^{r}\left(t, t_{1}\right)-g_{k \sigma j}^{a}\left(t, t_{1}\right)\right] G_{\sigma \sigma}^{<}\left(t_{1}, t^{\prime}\right)\right. \\
\left.+g_{k \sigma j}^{<}\left(t, t_{1}\right)\left[G_{\sigma \sigma}^{a}\left(t_{1}, t^{\prime}\right)-G_{\sigma \sigma}^{r}\left(t_{1}, t^{\prime}\right)\right]\right\} .
\end{gathered}
$$

Efetuando transformada de Fourier vem

$$
\begin{aligned}
I_{j}=-\frac{e}{\hbar} \sum_{k \sigma}\left|t_{k \sigma j}\right|^{2} \int \frac{d \epsilon}{2 \pi}\{[ & \left.g_{k \sigma j}^{r}(\epsilon)-g_{k \sigma j}^{a}(\epsilon)\right] G_{\sigma \sigma}^{<}(\epsilon) \\
& \left.+g_{k \sigma j}^{<}(\epsilon)\left[G_{\sigma \sigma}^{a}(\epsilon)-G_{\sigma \sigma}^{r}(\epsilon)\right]\right\} .
\end{aligned}
$$

Sendo

$$
g_{k \sigma j}^{r}(\epsilon)-g_{k \sigma j}^{a}(\epsilon)=\frac{1}{\epsilon-\epsilon_{k \sigma j}+i \delta}-\frac{1}{\epsilon-\epsilon_{k \sigma j}-i \delta}=-2 \pi i \delta\left(\epsilon-\epsilon_{k \sigma j}\right)
$$

e

$$
g_{k \sigma j}^{<}(\epsilon)=2 \pi i n_{j}(\epsilon) \delta\left(\epsilon-\epsilon_{k \sigma j}\right)
$$

onde $n_{j}(\epsilon)$ é a função de Fermi do $j$-ésimo terminal [57], obtemos para a corrente

$$
I_{j}=\frac{i e}{\hbar} \int \frac{d \epsilon}{2 \pi} \sum_{\sigma} \Gamma_{\sigma}^{j}\left\{G_{\sigma \sigma}^{<}(\epsilon)+n_{j}(\epsilon)\left[G_{\sigma \sigma}^{r}(\epsilon)-G_{\sigma \sigma}^{a}(\epsilon)\right]\right\},
$$

onde $\Gamma_{\sigma}^{j}=2 \pi \sum_{k}\left|t_{k \sigma j}\right|^{2} \delta\left(\epsilon-\epsilon_{k \sigma j}\right)$. Seguimos calculando as funções de Green do ponto quântico, $G_{\sigma \sigma}^{<}, G_{\sigma \sigma}^{r}$ e $G_{\sigma \sigma}^{a}$, que são necessárias para o cálculo da corrente.

\subsection{Funções de Green do ponto quântico}

As funções de Green do ponto quântico $G_{\sigma \sigma}^{<}, G_{\sigma \sigma}^{r}$ e $G_{\sigma \sigma}^{a}$ são obtidas a partir de $G_{\sigma \sigma}\left(\tau, \tau^{\prime}\right)=-i\left\langle T_{C} d_{\sigma}(\tau) d_{\sigma}^{\dagger}\left(\tau^{\prime}\right)\right\rangle$ via continuação analítica. Para encontrar$\operatorname{mos} G_{\sigma \sigma}\left(\tau, \tau^{\prime}\right)$ seguimos o procedimento padrão das funções de Green de não equilíbrio resumido no capítulo 2 , isto é, obtemos uma equação para $G_{\sigma \sigma}^{t}\left(t, t^{\prime}\right)=$ $-i\left\langle T d_{\sigma}(t) d_{\sigma}^{\dagger}\left(t^{\prime}\right)\right\rangle$, em seguida escrevemos uma equação idêntica para a função de Green com ordenamento ao longo do contorno. Vale lembrar que esse mesmo procedimento já foi adotado na Sec.3.7. 
Calculando a equação de movimento para a função de Green $G_{\sigma \sigma}^{t}\left(t, t^{\prime}\right)=$ $-i\left\langle T d_{\sigma}(t) d_{\sigma}^{\dagger}\left(t^{\prime}\right)\right\rangle$ obtemos

$$
\left[i \frac{\partial}{\partial t}-\epsilon_{\sigma}\right] G_{\sigma \sigma}^{t}\left(t, t^{\prime}\right)=\delta\left(t-t^{\prime}\right)+\int d t_{1} \Sigma_{\sigma \sigma}^{t}\left(t, t_{1}\right) G_{\sigma \sigma}^{t}\left(t_{1}, t^{\prime}\right)
$$

onde $\Sigma_{\sigma \sigma}^{t}\left(t, t_{1}\right)=\sum_{k j}\left|t_{k \sigma j}\right|^{2} g_{k \sigma j}^{t}\left(t, t_{1}\right)$, sendo $g_{k \sigma j}^{t}\left(t, t_{1}\right)=-i\left\langle T \widetilde{c}_{k \sigma j}(t) \widetilde{c}_{k \sigma j}^{\dagger}\left(t^{\prime}\right)\right\rangle$. Definindo a função de Green do ponto quântico "livre"

$$
\left[i \frac{\partial}{\partial t}-\epsilon_{\sigma}\right] G_{\sigma \sigma}^{0 t}\left(t, t^{\prime}\right)=\delta\left(t-t^{\prime}\right)
$$

isto é, $G_{\sigma \sigma}^{0 t}\left(t, t^{\prime}\right)$ é a função de Green de um ponto quântico sem acoplamento a contatos e sem interação de Coulomb, podemos reescrever a Eq.(5.10) na forma de uma equação de Dyson

$$
G_{\sigma \sigma}^{t}\left(t, t^{\prime}\right)=G_{\sigma \sigma}^{0 t}\left(t, t^{\prime}\right)+\iint d t_{1} d t_{2} G_{\sigma \sigma}^{0 t}\left(t, t_{2}\right) \Sigma_{\sigma \sigma}^{t}\left(t_{2}, t_{1}\right) G_{\sigma \sigma}^{t}\left(t_{1}, t^{\prime}\right) .
$$

Reescrevendo essa mesma equação com tempos ordenados ao longo do contorno, vem

$$
G_{\sigma \sigma}^{t}\left(\tau, \tau^{\prime}\right)=G_{\sigma \sigma}^{0 t}\left(\tau, \tau^{\prime}\right)+\iint d \tau_{1} d \tau_{2} G_{\sigma \sigma}^{0 t}\left(\tau, \tau_{2}\right) \Sigma_{\sigma \sigma}^{t}\left(\tau_{2}, \tau_{1}\right) G_{\sigma \sigma}^{t}\left(\tau_{1}, \tau^{\prime}\right) .
$$

Agora, aplicando as regras de Langreth e transformada de Fourier, obtemos as seguintes funções de Green

$$
\begin{aligned}
G_{\sigma \sigma}^{r}(\epsilon) & =G_{\sigma \sigma}^{0 r}(\epsilon)+G_{\sigma \sigma}^{0 r}(\epsilon) \Sigma_{\sigma \sigma}^{r}(\epsilon) G_{\sigma \sigma}^{r}(\epsilon) \Rightarrow G_{\sigma \sigma}^{r}(\epsilon)=\frac{1}{G_{\sigma \sigma}^{0^{-1}}-\Sigma_{\sigma}^{r}}, \\
G_{\sigma \sigma}^{<}(\epsilon) & =G_{\sigma \sigma}^{r}(\epsilon) \Sigma_{\sigma \sigma}^{<}(\epsilon) G_{\sigma \sigma}^{a}(\epsilon)
\end{aligned}
$$

onde $G_{\sigma \sigma}^{0^{-1}}=\epsilon-\epsilon_{\sigma}$. As equações (5.14) e (5.15) são as equações de Dyson e de Keldysh, respectivamente. Analisemos agora com maior detalhe as auto-energias $\Sigma_{\sigma \sigma}^{r}(\epsilon)$ e $\Sigma_{\sigma \sigma}^{<}(\epsilon)$.

\subsubsection{Auto-energias}

Para a auto-energia retardada $\Sigma_{\sigma \sigma}^{r}(\epsilon)$ temos a seguinte expressão

$$
\begin{aligned}
\Sigma_{\sigma}^{r}(\epsilon) & =\sum_{k j}\left|t_{k \sigma j}\right|^{2} g_{k \sigma j}^{r}(\epsilon)=\sum_{j} \sum_{k}\left|t_{k \sigma j}\right|^{2} \frac{1}{\epsilon-\epsilon_{k \sigma j}+i \delta} \\
& =\sum_{j} \int d \epsilon_{k}\left|t_{k \sigma j}\right|^{2} \frac{\rho\left(\epsilon_{k \sigma j}\right)}{\epsilon-\epsilon_{k \sigma j}+i \delta},
\end{aligned}
$$

onde $\rho\left(\epsilon_{k \sigma j}\right)$ é a densidade de estados do $j$-ésimo terminal. Para uma densidade de estados constante, $\rho\left(\epsilon_{k \sigma j}\right)=\rho_{j \sigma}=$ cte, e $t_{k \sigma j}$ também constante (wide-band limit [7]), obtemos

$$
\Sigma_{\sigma \sigma}^{r}(\epsilon)=-\sum_{j} i \pi|t|^{2} \rho_{j \sigma} \int d \epsilon_{k} \delta\left(\epsilon-\epsilon_{k \sigma j}\right)=-\frac{i}{2} \sum_{j} \Gamma_{\sigma}^{j},
$$


onde $\Gamma_{\sigma}^{j}=2 \pi|t|^{2} \rho_{j \sigma}$. Para a auto-energia menor temos

$$
\Sigma_{\sigma \sigma}^{<}(\epsilon)=\sum_{k j}|t|^{2} g_{k \sigma j}^{<}(\epsilon)=\sum_{k j}|t|^{2} 2 \pi i n_{j}(\epsilon) \delta\left(\epsilon-\epsilon_{k \sigma j}\right)=i \sum_{j} n_{j}(\epsilon) \Gamma_{\sigma}^{j} \cdot(5.1
$$

Vale observar que as auto-energias (5.17) e (5.18) são generalizações das autoenergias do caso particular de dois terminais acoplados a um ponto quântico, isto é, $\Sigma_{\sigma}^{r}=-\frac{i}{2}\left[\Gamma_{\sigma}^{L}+\Gamma_{\sigma}^{R}\right]$, e $\Sigma_{\sigma}^{<}=i\left[\Gamma_{\sigma}^{L} n_{L}+\Gamma_{\sigma}^{R} n_{R}\right]$, onde os índices $L$ e $R$ indicam os terminais da esquerda (left) e da direita (right), respectivamente [7].

Com essas auto-energias as funções de Green (5.14) e (5.15) podem ser expressas como segue na próxima subseção.

\subsubsection{Função de Green retardada e equação de Keldysh}

Substituindo a Eq.(5.17) na Eq.(5.14) obtemos para a função de Green retardada o seguinte

$$
G_{\sigma \sigma}^{r}(\epsilon)=\frac{1}{G_{\sigma \sigma}^{0^{-1}}+\frac{i}{2} \sum_{j} \Gamma_{\sigma}^{j}}=\frac{1}{\epsilon-\epsilon_{\sigma}+\frac{i}{2} \sum_{j} \Gamma_{\sigma}^{j}} .
$$

A função de Green avançada é dada por $G^{a}=G^{r^{*}}$, sendo $G^{r^{*}}$ o complexo conjugado de $G^{r}$. Note que

$$
\begin{aligned}
G_{\sigma \sigma}^{r}(\epsilon)-G_{\sigma \sigma}^{a}(\epsilon) & =\frac{1}{\epsilon-\epsilon_{\sigma}+\frac{i}{2} \sum_{j} \Gamma_{\sigma}^{j}}-\frac{1}{\epsilon-\epsilon_{\sigma}-\frac{i}{2} \sum_{j} \Gamma_{\sigma}^{j}} \\
& =\frac{-i \sum_{j} \Gamma_{\sigma}^{j}}{\left(\epsilon-\epsilon_{\sigma}\right)^{2}+\left(\frac{1}{2} \sum_{j} \Gamma_{\sigma}^{j}\right)^{2}}=-i \sum_{j} \Gamma_{\sigma}^{j} G_{\sigma \sigma}^{r} G_{\sigma \sigma}^{a}
\end{aligned}
$$

que será uma equação útil em cálculos subseqüentes. Para obtermos a função de Green menor devemos substituir a Eq.(5.18) na Eq.(5.15). Com isso vem

$$
G_{\sigma \sigma}^{<}=i G_{\sigma \sigma}^{r} \sum_{j} n_{j}(\epsilon) \Gamma_{\sigma}^{j} G_{\sigma \sigma}^{a}=-\frac{\sum_{j} n_{j}(\epsilon) \Gamma_{\sigma}^{j}}{\sum_{j} \Gamma_{\sigma}^{j}}\left[G_{\sigma \sigma}^{r}(\epsilon)-G_{\sigma \sigma}^{a}(\epsilon)\right],
$$

onde o último membro foi obtido utilizando a Eq.(5.20).

\subsection{Forma explícita da corrente}

Substituindo a função de Green menor (5.21) na equação da corrente (5.9) obtemos

$$
\begin{aligned}
I_{j} & =\frac{i e}{\hbar} \int \frac{d \epsilon}{2 \pi} \sum_{\sigma} \Gamma_{\sigma}^{j}\left[n_{j}(\epsilon)-\frac{\sum_{l} n_{l}(\epsilon) \Gamma_{\sigma}^{l}}{\sum_{l} \Gamma_{\sigma}^{l}}\right]\left[G_{\sigma \sigma}^{r}(\epsilon)-G_{\sigma \sigma}^{a}(\epsilon)\right] \\
& =\frac{i e}{\hbar} \int \frac{d \epsilon}{2 \pi} \sum_{\sigma} \frac{\sum_{l}\left[n_{j}(\epsilon)-n_{l}(\epsilon)\right] \Gamma_{\sigma}^{l} \Gamma_{\sigma}^{j}}{\sum_{l} \Gamma_{\sigma}^{l}}\left[G_{\sigma \sigma}^{r}(\epsilon)-G_{\sigma \sigma}^{a}(\epsilon)\right] .
\end{aligned}
$$


Essa equação é equivalente a expressão de C. A. Stafford [86] [veja nota de rodapé] para um sistema de múltiplos terminais. Porém ressaltamos que em [86] não há efeitos dependentes de spin, visto que os terminais não são magnéticos ${ }^{1}$.

Definindo um coeficiente de transmissão $T_{j l}^{\sigma}=\frac{\Gamma_{\sigma}^{j} \Gamma_{\sigma}^{l}}{\Gamma_{\sigma}} A_{\sigma}(\epsilon)$, onde $\Gamma_{\sigma}=\sum_{l} \Gamma_{\sigma}^{l} \mathrm{e}$ $A(\epsilon)=i\left[G_{\sigma \sigma}^{r}(\epsilon)-G_{\sigma \sigma}^{a}(\epsilon)\right]$, podemos escrever a corrente como segue

$$
I_{j}=\frac{e}{2 \pi \hbar} \int d \epsilon \sum_{\sigma l} T_{j l}^{\sigma}\left(n_{j}-n_{l}\right),
$$

que é a fórmula de Landauer-Büttiker [64] generalizada para um problema de múltiplos terminais. Note que o elemento de matriz $T_{j l}^{\sigma} \propto \Gamma_{\sigma}^{j} A_{\sigma} \Gamma_{\sigma}^{l}$ contém informação das densidades de estado dos reservatórios $j$ e $l$, dadas por $\Gamma_{\sigma}^{j}$ e $\Gamma_{\sigma}^{l}$, e da densidade de estados do ponto quântico, dada por $A_{\sigma}$.

A Eq.(5.23) fornece o resultado bem conhecido para o sistema de dois terminais. Consideremos $j=L$ (terminal da esquerda) e $l=L, R$, sendo $R$ o índice para o terminal da direita. Com isso vem

$$
I_{L}=\frac{e}{\hbar} \int \frac{d \epsilon}{2 \pi} \sum_{\sigma} T_{L R}^{\sigma}\left(n_{L}-n_{R}\right) .
$$

Sendo $T_{L R}^{\sigma}=\left(\Gamma_{\sigma}^{L} \Gamma_{\sigma}^{R}\right) /\left(\Gamma_{\sigma}^{L}+\Gamma_{\sigma}^{R}\right) i\left[G_{\sigma \sigma}^{r}(\epsilon)-G_{\sigma \sigma}^{a}(\epsilon)\right]$, obtemos

$$
\begin{aligned}
T_{L R}^{\sigma} & =\frac{\Gamma_{\sigma}^{L} \Gamma_{\sigma}^{R}}{\Gamma_{\sigma}^{L}+\Gamma_{\sigma}^{R}} i\left\{\frac{1}{\epsilon-\epsilon_{\sigma}+i \frac{\Gamma_{\sigma}^{L}+\Gamma_{\sigma}^{R}}{2}}-\frac{1}{\epsilon-\epsilon_{\sigma}-i \frac{\Gamma_{\sigma}^{L}+\Gamma_{\sigma}^{R}}{2}}\right\} \\
& =\frac{\Gamma_{\sigma}^{L} \Gamma_{\sigma}^{R}}{\Gamma_{\sigma}^{L}+\Gamma_{\sigma}^{R}} i \frac{-i\left(\Gamma_{\sigma}^{L}+\Gamma_{\sigma}^{R}\right)}{\left(\epsilon-\epsilon_{\sigma}\right)^{2}+\left(\frac{\Gamma_{\sigma}^{L}+\Gamma_{\sigma}^{R}}{2}\right)^{2}} \\
& =\frac{\Gamma_{\sigma}^{L} \Gamma_{\sigma}^{R}}{\left(\epsilon-\epsilon_{\sigma}\right)^{2}+\left(\frac{\Gamma_{\sigma}^{L}+\Gamma_{\sigma}^{R}}{2}\right)^{2}},
\end{aligned}
$$

que é a fórmula de Breit-Wigner para o coeficiente de transmissão [64],[87]. Com isso obtemos para a corrente o seguinte resultado

$$
I_{L}=\frac{e}{2 \pi \hbar} \int d \epsilon \sum_{\sigma} \frac{\Gamma_{\sigma}^{L} \Gamma_{\sigma}^{R}}{\left(\epsilon-\epsilon_{\sigma}\right)^{2}+\left(\frac{\Gamma_{\sigma}^{L}+\Gamma_{\sigma}^{R}}{2}\right)^{2}}\left(n_{L}-n_{R}\right) .
$$

Agora considerando o regime de alta voltagem, isto é, $\epsilon_{\sigma}$ em total ressonância com os estados do emissor e acima dos estados do coletor $\left(\mu_{R}<<\epsilon_{\sigma}<<\mu_{L}\right)$, podemos fazer $n_{L}=1$ e $n_{R}=0$ na Eq.(5.26), e com isso encontramos

$$
I_{L}=\frac{e}{2 \pi \hbar} \sum_{\sigma} \Gamma_{\sigma}^{L} \Gamma_{\sigma}^{R} \int_{-\infty}^{\infty} d \epsilon \frac{1}{\left(\epsilon-\epsilon_{\sigma}\right)^{2}+\left(\frac{\Gamma_{\sigma}^{L}+\Gamma_{\sigma}^{R}}{2}\right)^{2}}=\frac{e}{\hbar} \sum_{\sigma} \frac{\Gamma_{\sigma}^{L} \Gamma_{\sigma}^{R}}{\Gamma_{\sigma}^{L}+\Gamma_{\sigma}^{R}} .
$$

A equação (5.27) é bastante familiar dentro da teoria de transporte por tunelamento ressonante [64]. Vale ressaltar que essa equação é válida na condição de ressonância, e na ausência de interação de Coulomb e de espalhamento de spin.

\footnotetext{
${ }^{1}$ Stafford também estudou o problema de um ponto quântico acoplado a três terminais [86]. Porém em seu modelo os terminais não são ferromagnéticos e o transporte se dá somente entre dois terminais. O terceiro terminal opera apenas como um medidor de voltagem (voltage probe).
} 


\subsection{Resposta linear}

Analisemos agora a equação (5.23) no regime de baixas tensões. Comecemos expandindo a função de Fermi nesse regime,

$$
n_{j}\left(\epsilon, \mu_{j}\right)=n_{j}\left(\epsilon, \mu_{j}^{0}\right)+\left.\frac{\partial n_{j}}{\partial \mu_{j}}\right|_{\mu_{j}=\mu_{j}^{0}}\left(\mu_{j}-\mu_{j}^{0}\right)=n_{j}\left(\epsilon, \mu_{j}^{0}\right)-\frac{\partial n_{j}^{0}}{\partial \epsilon}\left(\mu_{j}-\mu_{j}^{0}\right)
$$

onde $\mu_{j}=\mu_{j}^{0}+\Phi_{j}$, sendo $\mu_{j}^{0}$ o potencial químico do $j$-ésimo eletrodo sem tensão externa e $\phi_{j}=-e V_{j}$. Substituindo essa expansão na equação da corrente (5.23) obtemos

$$
I_{j}=\frac{e}{2 \pi \hbar} \int d \epsilon \sum_{\sigma l} T_{j l}^{\sigma} \frac{\partial n_{j}^{0}}{\partial \epsilon}\left(\mu_{l}-\mu_{j}\right)
$$

Como em baixas temperaturas temos $\frac{\partial n_{j}^{0}}{\partial \epsilon}=-\delta\left(\epsilon-\mu_{j}^{0}\right)$, a Eq.(5.29) pode ser expressa assim

$$
I_{j}=\frac{e}{h} \sum_{\sigma l} T_{j l}^{\sigma}\left(\mu_{j}^{0}\right)\left(\mu_{j}-\mu_{l}\right)
$$

que é a fórmula de Landauer-Büttiker para estruturas com múltiplos terminais [88].

\subsection{Sistema envolvendo três terminais}

Agora apliquemos a Eq.(5.23) a um sistema constituído por três terminais ferromagnéticos acoplados ao ponto quântico, conforme ilustramos na figura (5.2). Consideramos que o terminal (1) é o emissor de elétrons, e os terminais (2) e (3) são coletores, ou seja, assumimos $\mu_{1}>\mu_{2}, \mu_{3}$. As configurações magnéticas dos terminais são as seguintes: o emissor é $\uparrow$-polarizado (não necessariamente $100 \%$ polarizado), e os coletores têm magnetizações que podem variar entre $\uparrow$ e $\downarrow$, independentemente uma da outra. Na configuração denotada por (1) (setas pretas nos coletores) temos os três terminais alinhados paralelamente. Na configuração (2) (setas vermelhas) os dois terminais coletores estão alinhados paralelamente entre si, e anti-paralelamente alinhados com relação ao terminal emissor. Na terceira configuração (setas azuis) o coletor (2) tem sua magnetização alinhada paralelamente a magnetização do emissor, enquanto o coletor (3) alinha-se anti-paralelamente ao emissor. Portanto na configuração (3) as magnetizações dos coletores são antiparalelas entre si. 


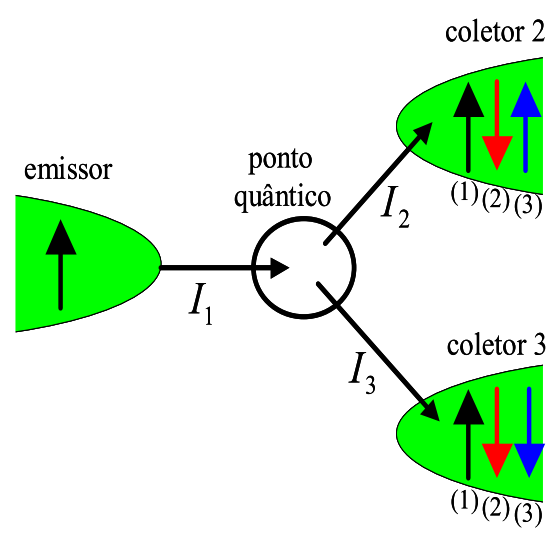

Figura 5.2: Sistema constituído por três terminais ferromagnéticos acoplados a um ponto quântico. O emissor possui uma magnetização fixa $\uparrow$, enquanto os reservatórios coletores (2) e (3) tem magnetizações variáveis entre $\uparrow \mathrm{e} \downarrow$. As três configurações magnéticas analisadas estão indicadas pelas setas nos coletores. Na configuração (1) temos todos os terminais alinhados paralelamente entre si. Na configuração (2) os coletores são alinhados anti-paralelamente com o emissor, e na configuração (3) temos um coletor alinhado paralelamente ao emissor e o outro alinhado anti-paralelamente ao emissor. 
Em analogia ao sistema de dois terminais ferromagnéticos discutidos no capítulo anterior, onde tínhamos $\Gamma_{\sigma}^{L}=\Gamma_{0}\left[1+(-1)^{\delta_{\downarrow \sigma}} p_{L}\right]$ e $\Gamma_{\sigma}^{R}=\Gamma_{0}\left[1 \pm(-1)^{\delta_{\downarrow \sigma}} p_{R}\right]$, no presente caso envolvendo três terminais, adotamos os seguintes parâmetros

$$
\begin{aligned}
\Gamma_{\sigma}^{(1)} & =\Gamma_{0}\left[1+(-1)^{\delta_{\downarrow}} p_{1}\right] \\
\Gamma_{\sigma}^{(2)} & =\Gamma_{0}\left[1 \pm(-1)^{\delta_{\downarrow} \sigma} p_{2}\right] \\
\Gamma_{\sigma}^{(3)} & =\Gamma_{0}\left[1 \pm(-1)^{\delta_{\downarrow}} p_{3}\right]
\end{aligned}
$$

onde os sinais + e - que aparecem em $\Gamma_{\sigma}^{(2)}$ e $\Gamma_{\sigma}^{(3)}$ correspondem aos alinhamentos paralelo e anti-paralelo, respectivamente, com relação ao terminal emissor. O emissor é o nosso referencial de polarização magnética, isto é, mantemos sua orientação magnética fixa, e variamos as orientações magnéticas dos coletores, passando de polarização paralela a anti-paralela, como ilustrado na Fig.(5.2).

Aplicando a Eq.(5.23) para o sistema de três terminais, obtemos a seguinte expressão para a corrente no terminal (1)

$$
\begin{aligned}
I_{1} & =\frac{e}{\hbar} \int \frac{d \epsilon}{2 \pi} \sum_{\sigma}\left[T_{12}^{\sigma}\left(n_{1}-n_{2}\right)+T_{13}^{\sigma}\left(n_{1}-n_{3}\right)\right] \\
& =\frac{i e}{\hbar} \int \frac{d \epsilon}{2 \pi} \sum_{\sigma} \frac{\Gamma_{\sigma}^{(1)}}{\Gamma_{\sigma}^{(1)}+\Gamma_{\sigma}^{(2)}+\Gamma_{\sigma}^{(3)}}\left[\Gamma_{\sigma}^{(2)}\left(n_{1}-n_{2}\right)+\Gamma_{\sigma}^{(3)}\left(n_{1}-n_{3}\right)\right]\left[G_{\sigma \sigma}^{r}(\epsilon)-G_{\sigma \sigma}^{a}(\epsilon)\right] \\
& =\frac{e}{\hbar} \int \frac{d \epsilon}{2 \pi} \sum_{\sigma} \frac{\Gamma_{\sigma}^{(1)}\left[\Gamma_{\sigma}^{(2)}\left(n_{1}-n_{2}\right)+\Gamma_{\sigma}^{(3)}\left(n_{1}-n_{3}\right)\right]}{\left(\epsilon-\epsilon_{\sigma}\right)^{2}+\left(\frac{\Gamma_{\sigma}^{(1)}+\Gamma_{\sigma}^{(2)}+\Gamma_{\sigma}^{(3)}}{2}\right)^{2}} .
\end{aligned}
$$

Analogamente temos para o terminal (2)

$$
I_{2}=\frac{e}{\hbar} \int \frac{d \epsilon}{2 \pi} \sum_{\sigma} \frac{\Gamma_{\sigma}^{(2)}\left[\Gamma_{\sigma}^{(1)}\left(n_{2}-n_{1}\right)+\Gamma_{\sigma}^{(3)}\left(n_{2}-n_{3}\right)\right]}{\left(\epsilon-\epsilon_{\sigma}\right)^{2}+\left(\frac{\Gamma_{\sigma}^{(1)}+\Gamma_{\sigma}^{(2)}+\Gamma_{\sigma}^{(3)}}{2}\right)^{2}},
$$

e para o terminal (3)

$$
I_{3}=\frac{e}{\hbar} \int \frac{d \epsilon}{2 \pi} \sum_{\sigma} \frac{\Gamma_{\sigma}^{(3)}\left[\Gamma_{\sigma}^{(1)}\left(n_{3}-n_{1}\right)+\Gamma_{\sigma}^{(2)}\left(n_{3}-n_{2}\right)\right]}{\left(\epsilon-\epsilon_{\sigma}\right)^{2}+\left(\frac{\Gamma_{\sigma}^{(1)}+\Gamma_{\sigma}^{(2)}+\Gamma_{\sigma}^{(3)}}{2}\right)^{2}} .
$$

Vamos considerar que os terminais (2) e (3) possuem o mesmo potencial químico, $\mu_{2}=\mu_{3}$. Além disso, tomemos como referencial de energia o potencial químico $\mu_{1}$, de modo a fazermos $\mu_{2}=\mu_{3}=\mu_{1}-e V$. Como $\mu_{2}=\mu_{3}$, não há corrente líquida entre os terminais (2) e (3), visto que esses terminais estão em equilíbrio entre si. A corrente flui apenas entre os terminais (1)-(2) e (1)-(3) quando $e V \neq 0$. Como $n_{2}=n_{3}$ (em virtude de $\mu_{2}=\mu_{3}$ ), as equações anteriores se reduzem a

$$
\begin{aligned}
& I_{1}=\frac{e}{\hbar} \int \frac{d \epsilon}{2 \pi} \sum_{\sigma} \frac{\Gamma_{\sigma}^{(1)}\left[\Gamma_{\sigma}^{(2)}\left(n_{1}-n_{2}\right)+\Gamma_{\sigma}^{(3)}\left(n_{1}-n_{3}\right)\right]}{\left(\epsilon-\epsilon_{\sigma}\right)^{2}+\left(\frac{\Gamma_{\sigma}^{(1)}+\Gamma_{\sigma}^{(2)}+\Gamma_{\sigma}^{(3)}}{2}\right)^{2}} \\
& I_{2}=\frac{e}{\hbar} \int \frac{d \epsilon}{2 \pi} \sum_{\sigma} \frac{\Gamma_{\sigma}^{(2)} \Gamma_{\sigma}^{(1)}\left(n_{2}-n_{1}\right)}{\left(\epsilon-\epsilon_{\sigma}\right)^{2}+\left(\frac{\Gamma_{\sigma}^{(1)}+\Gamma_{\sigma}^{(2)}+\Gamma_{\sigma}^{(3)}}{2}\right)^{2}} \\
& I_{3}=\frac{e}{\hbar} \int \frac{d \epsilon}{2 \pi} \sum_{\sigma} \frac{\Gamma_{\sigma}^{(3)} \Gamma_{\sigma}^{(1)}\left(n_{3}-n_{1}\right)}{\left(\epsilon-\epsilon_{\sigma}\right)^{2}+\left(\frac{\Gamma_{\sigma}^{(1)}+\Gamma_{\sigma}^{(2)}+\Gamma_{\sigma}^{(3)}}{2}\right)^{2}} .
\end{aligned}
$$


Note que $I_{1}+I_{2}+I_{3}=0$, indicando que há conservação de carga no sistema, isto é, a corrente que sai do emissor é igual a soma das correntes que entram nos reservatórios coletores. Essa lei de conservação se aplica também para cada componente de spin isoladamente, visto que não há espalhamento de spin no sistema.

Os resultados numéricos apresentados nesse capítulo foram obtidos via integração das Eqs.(5.37)-(5.39). Os valores dos vários parâmetros $\Gamma_{\sigma}^{(i)}$ que aparecem nessas equações (5.37)-(5.39), serão apresentados posteriormente. Seguimos resolvendo as equações (5.37)-(5.39) para um regime particular de tensão.

\subsubsection{Corrente no sistema de três terminais}

Nesta seção derivamos expressões para as correntes $I_{1}, I_{2}$ e $I_{3}$ no regime em que o nível do ponto quântico $\epsilon_{0}$ está inteiramente dentro da janela de condução do sistema, isto é, abaixo de $\mu_{1}$ e acima de $\mu_{2}=\mu_{3}$, portanto trata-se de um regime de alta voltagem. Nesse regime podemos adotar $n_{1}=1$ e $n_{2}=n_{3}=0$, nas equações (5.37)-(5.39), resultando assim nas seguintes expressões,

$$
\begin{aligned}
& I_{1}=\frac{e}{\hbar} \int_{-\infty}^{\infty} \frac{d \epsilon}{2 \pi} \sum_{\sigma} \frac{\Gamma_{\sigma}^{(1)}\left[\Gamma_{\sigma}^{(2)}+\Gamma_{\sigma}^{(3)}\right]}{\left(\epsilon-\epsilon_{\sigma}\right)^{2}+\left(\frac{\Gamma_{\sigma}^{(1)}+\Gamma_{\sigma}^{(2)}+\Gamma_{\sigma}^{(3)}}{2}\right)^{2}}, \\
& I_{2}=-\frac{e}{\hbar} \int_{-\infty}^{\infty} \frac{d \epsilon}{2 \pi} \sum_{\sigma} \frac{\Gamma_{\sigma}^{(2)} \Gamma_{\sigma}^{(1)}}{\left(\epsilon-\epsilon_{\sigma}\right)^{2}+\left(\frac{\Gamma_{\sigma}^{(1)}+\Gamma_{\sigma}^{(2)}+\Gamma_{\sigma}^{(3)}}{2}\right)^{2}}, \\
& I_{3}=-\frac{e}{\hbar} \int_{-\infty}^{\infty} \frac{d \epsilon}{2 \pi} \sum_{\sigma} \frac{\Gamma_{\sigma}^{(3)} \Gamma_{\sigma}^{(1)}}{\left(\epsilon-\epsilon_{\sigma}\right)^{2}+\left(\frac{\Gamma_{\sigma}^{(1)}+\Gamma_{\sigma}^{(2)}+\Gamma_{\sigma}^{(3)}}{2}\right)^{2}} .
\end{aligned}
$$

Como em nosso modelo os parâmetros $\Gamma_{\sigma}^{(i)}$ são constantes, isto é, não variam com a energia, podemos simplesmente efetuar a integral da Lorentziana que aparece nas três equações (5.40)-(5.42), isto é,

$$
\int_{-\infty}^{\infty} d \epsilon \frac{1}{\left(\epsilon-\epsilon_{\sigma}\right)^{2}+\left(\frac{\Gamma_{\sigma}^{(1)}+\Gamma_{\sigma}^{(2)}+\Gamma_{\sigma}^{(3)}}{2}\right)}=\frac{2 \pi}{\Gamma_{\sigma}^{(1)}+\Gamma_{\sigma}^{(2)}+\Gamma_{\sigma}^{(3)}}
$$

Com isso as correntes ficam

$$
\begin{aligned}
& I_{1}=\frac{e}{\hbar} \sum_{\sigma} \frac{\Gamma_{\sigma}^{(1)}\left[\Gamma_{\sigma}^{(2)}+\Gamma_{\sigma}^{(3)}\right]}{\Gamma_{\sigma}^{(1)}+\Gamma_{\sigma}^{(2)}+\Gamma_{\sigma}^{(3)}}, \\
& I_{2}=-\frac{e}{\hbar} \sum_{\sigma} \frac{\Gamma_{\sigma}^{(2)} \Gamma_{\sigma}^{(1)}}{\Gamma_{\sigma}^{(1)}+\Gamma_{\sigma}^{(2)}+\Gamma_{\sigma}^{(3)}}, \\
& I_{3}=-\frac{e}{\hbar} \sum_{\sigma} \frac{\Gamma_{\sigma}^{(3)} \Gamma_{\sigma}^{(1)}}{\Gamma_{\sigma}^{(1)}+\Gamma_{\sigma}^{(2)}+\Gamma_{\sigma}^{(3)}} .
\end{aligned}
$$

Observe que essas equação são uma generalização do caso particular de dois terminais acoplados a um ponto quântico [Eq.(5.27)]. Vale lembrar que nas equações (5.44)-(5.46) consideramos o nível $\epsilon_{0}$ inteiramente em ressonância com os estados do emissor e acima do potencial químico dos reservatórios coletores. 


\subsection{Função Espectral}

Por vezes nos é útil conhecer a função espectral do ponto quântico, $A_{\sigma}(\epsilon)=$ $i\left[G_{\sigma \sigma}^{r}(\epsilon)-G_{\sigma \sigma}^{a}(\epsilon)\right]$, para entendermos melhor o transporte. Utilizando a diferença $G_{\sigma \sigma}^{r}(\epsilon)-G_{\sigma \sigma}^{a}(\epsilon)$, calculada na Eq.(5.20), a função espectral para o sistema de três terminais assume a seguinte forma

$$
A_{\sigma}(\epsilon)=\frac{\Gamma_{\sigma}^{(1)}+\Gamma_{\sigma}^{(2)}+\Gamma_{\sigma}^{(3)}}{\left(\epsilon-\epsilon_{\sigma}\right)^{2}+\left(\frac{\Gamma_{\sigma}^{(1)}+\Gamma_{\sigma}^{(2)}+\Gamma_{\sigma}^{(3)}}{2}\right)^{2}} .
$$

Dessa equação vemos que o alargamento do nível $\epsilon_{\sigma}$ é dado pela soma dos parâmetros de acoplamento $\Gamma_{\sigma}^{(i)}$, portanto cada terminal tende a alargar um pouco mais o nível do ponto quântico.

\subsection{Parâmetros e metodologia de cálculo}

Os parâmetros utilizados aqui consistem de $\Gamma_{0}=10 \mu \mathrm{eV}$ [81], que dá a intensidade do acoplamento ponto-quântico-terminais [veja Eqs.(5.31)-(5.33)], o nível do ponto quântico $\epsilon_{\uparrow}=\epsilon_{\downarrow}=\epsilon_{d}=\epsilon_{0}-e V / 2$, e o potencial químico do reservatório emissor $\mu_{1}=0$, e dos coletores $\mu_{2}=\mu_{3}=\mu_{1}-e V$. A temperatura adotada foi $k_{B} T=$ $20 \mu \mathrm{eV}(T=232 \mathrm{mK})$. A tensão $e V$ é tipicamente da ordem de alguns meV, e $p_{i}$ varia entre 0 e 1 . Para $p_{i}=0$ temos $i$-ésimo terminal não magnetizado, e para $p_{i}=1$ a sua polarização é máxima (100\%).

O procedimento de cálculo é bem mais simples do que o utilizado no capítulo anterior. Aqui integramos as equações (5.37)-(5.39) para diferentes valores de tensão ou de polarização $p_{i}$. Como não incluímos interação de Coulomb, um cálculo auto-consistente não se faz necessário, ao contrário do que foi feito no capítulo 4.

\subsection{Resultados}

\subsubsection{Corrente e polarização versus voltagem}

A figura (5.3) mostra as correntes $I_{\uparrow}$ e $I_{\downarrow}$ versus tensão nos três terminais e nas três configurações magnéticas. Observamos que a corrente vale inicialmente zero $(e V \lesssim 1)$ e cresce rapidamente em torno de $e V \approx 1 \mathrm{meV}$. Esse aumento da corrente deve-se a ressonância do nível $\epsilon_{d}=0.5-\mathrm{eV} / 2$ com os estados do emissor-para $e V=1 \mathrm{meV}$ temos $\epsilon_{\sigma}=\mu_{1}=0$.

Após $\mathrm{eV} \approx 1 \mathrm{meV}$ as correntes $I_{\uparrow}$ e $I_{\downarrow}$ atingem plateaus, nos quais vemos claramente uma polarização $I_{\uparrow} \neq I_{\downarrow}$. Nas configurações 1 e 2 temos uma corrente $\uparrow$-polarizada $\left(I_{\uparrow}>I_{\downarrow}\right)$ em todos os terminais. Na configuração 3 a corrente sai do emissor $\uparrow$-polarizada, entra no coletor (2) também $\uparrow$-polarizada, porém entra 
$\downarrow$-polarizada no coletor (3). Com isso obtemos as duas polarizações $\uparrow$ e $\downarrow$ simultaneamente no sistema. Além disso, nessa configuração (3) temos que a polarização no terminal (2) intensifica-se com relação a polarização no terminal (1) [note que a diferença $I_{\uparrow}-I_{\downarrow}$ é maior no coletor (2) do que no emissor].

O fato de termos na configuração 3 corrente $\uparrow$-polarizada no coletor (2) e $\downarrow$ polarizada no coletor (3) não decorre trivialmente da magnetização $\uparrow$ do terminal (2) e $\downarrow$ do terminal (3). Em outras palavras, a polarização da corrente não necessariamente segue a polarização do terminal. Podemos, mesmo na configuração (3), ter uma corrente $\uparrow$-polarizada no terminal (3). A polarização da corrente depende do alinhamento magnético dos terminais e do valor dos parâmetros $p_{i}$. Portanto trata-se de um efeito combinado da magnetização dos eletrodos e de seus alinhamentos magnéticos. Posteriormente faremos diversos gráficos das correntes $I_{\uparrow}$ e $I_{\downarrow}$ como função dos vários $p_{i}$ 's no intuito de aprender como as correntes $I_{\uparrow} \mathrm{e}$ $I_{\downarrow}$ variam como função desses parâmetros.

$\mathrm{Na}$ figura (5.4) ilustramos a polarização versus tensão externa para as três configurações ilustradas na figura (5.3). Na configuração 1 temos que a polarização da corrente nos três terminais é a mesma, e essa diminui quando o sistema entra em ressonância $(e V \gtrsim 1) \mathrm{meV}$. Na configuração 2 a polarização encontra-se inicialmente $(e V \rightarrow 0)$ em torno de zero e aumenta na faixa de ressonância $(e V \gtrsim 1$ meV), sendo a polarização igual nos três terminais. Já na configuração 3 as polarizações nos vários terminais diferem entre si. Note que no terminal (2) temos uma amplificação da polarização frente a polarização no terminal (1), e uma supressão (e mudança de sinal) da polarização no terminal (3). Como a corrente pode ser expressa em termos da função espectral do ponto quântico $A_{\sigma}(\epsilon)$, podemos entender o comportamento dessas polarizações nas várias configurações, em parte através da função espectral $A_{\sigma}(\epsilon)$.

Na figura (5.5) ilustramos $A_{\sigma}(\epsilon)$ para as três configurações magnéticas consideradas. Na configuração 1 temos que as funções espectrais para spin $\uparrow$ e $\downarrow$ diferem em largura e amplitude. Assim, quando o nível $\epsilon_{\sigma}=0.5-\mathrm{eV} / 2$ começa a se aproximar de $\mu_{1}$, a corrente é predominantemente $\uparrow$, visto que $A_{\uparrow}(\epsilon)$ é mais largo do que $A(\epsilon)_{\downarrow}$. Observe na Fig.(5.3)-config.1 que a corrente $I_{\uparrow}$ começa primeiro do que $I_{\downarrow}$. Essa defasagem deve-se exatamente a diferença entre as larguras de $A_{\uparrow} \mathrm{e}$ $A_{\downarrow}$. Portanto, apesar de não existir nenhum desdobramento de $\operatorname{spin}\left(\epsilon_{\uparrow}=\epsilon_{\downarrow}\right)$, as correntes $I_{\uparrow}$ e $I_{\downarrow}$ podem iniciar em valores de tensão ligeiramente diferentes. Com o aumento da tensão, o nível $A_{\downarrow}(\epsilon)$ se aproxima mais de $\mu_{1}$, e conseqüentemente a corrente $I_{\downarrow}$ também aumenta, resultando na diminuição da polarização observada na Fig.5.4(a).

Na configuração 2 temos que $A_{\uparrow}(\epsilon)$ e $A_{\downarrow}(\epsilon)$ apresentam aproximadamente a mesma largura, e amplitudes diferentes $\left(A_{\uparrow}>A_{\downarrow}\right)$. Desse modo, em baixas tensões as corrente $I_{\uparrow}$ e $I_{\downarrow}$ são aproximadamente iguais, resultando em $\wp \approx 0$. Quando a tensão cresce e os estados do ponto quântico $A_{\uparrow}$ e $A_{\downarrow}$ entram em ressonância com 
os estados do emissor, a corrente $I_{\uparrow}$ cresce mais do que $I_{\downarrow}$ nos dois coletores em virtude de $A_{\uparrow}>A_{\downarrow}$. Isso leva ao aumento da polarização visto na Fig.5.4-config.2. Para a configuração 3 temos que $A_{\downarrow}>A_{\uparrow}$, o que explica em parte a polarização negativa da corrente no terceiro terminal [Fig.5.4(c)].

As correntes $I_{\uparrow}$ e $I_{\downarrow}$ nos vários terminais também foram estudadas como função de $p_{1}, p_{2}$ e $p_{3}$, conforme discutimos na próxima seção. 

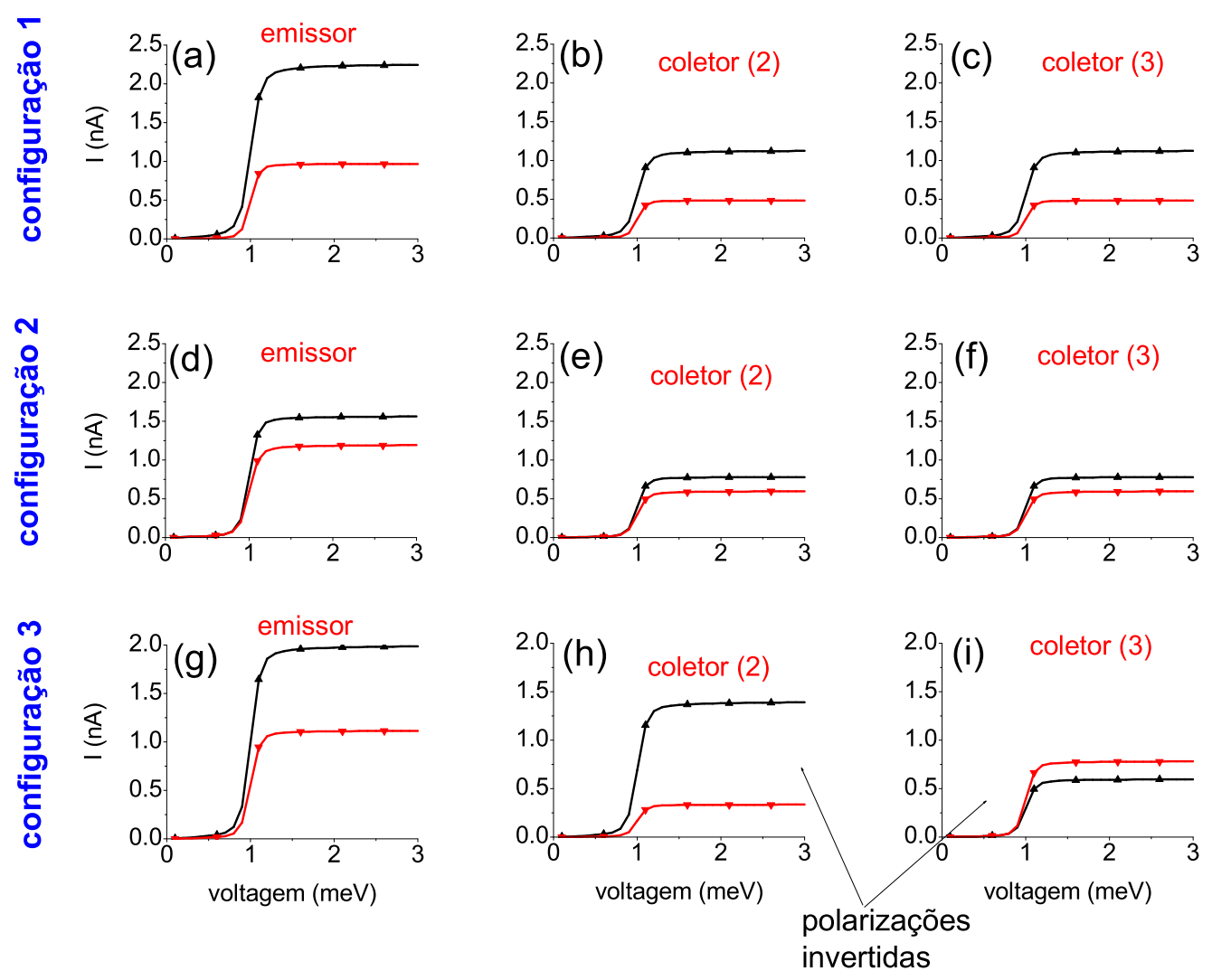

Figura 5.3: Correntes $I_{\uparrow}(\Delta)$ e $I_{\downarrow}(\nabla)$ como função da tensão externa, nos três terminais e nas três configurações magnéticas ilustradas na Fig.(5.2). Em todos os painéis as correntes valem inicialmente zero $(e V \lesssim 1 \mathrm{meV})$ e sobem rapidamente em torno de $e V \approx 1 \mathrm{meV}$, quando o nível $\epsilon_{\uparrow}=\epsilon_{\downarrow}=0.5-\mathrm{eV} / 2$ entra em ressonância com os estados do emissor $\left(\epsilon_{\uparrow}=\epsilon_{\downarrow}=\mu_{1}\right)$. Após $e V \approx 1 \mathrm{meV}$ as correntes $I_{\uparrow}$ e $I_{\downarrow}$ atingem plateaus nos quais temos $I_{\uparrow} \neq I_{\downarrow}$, e portanto uma polarização não nula. Nas configurações 1 e 2 [(a)-(f)] as correntes são todas $\uparrow$-polarizadas $\left(I_{\uparrow}>I_{\downarrow}\right)$. Porém, na configuração 3 temos que a polarização no emissor e no coletor $(2)[(\mathrm{g})-(\mathrm{h})]$ é positiva $\left(I_{\uparrow}>I_{\downarrow}\right)$, e no coletor 3 [painel (i)] negativa $\left(I_{\uparrow}<I_{\downarrow}\right)$. Essa polarização negativa no coletor $(3)$ deve-se ao efeito combinado do alinhamento magnético dos terminais e dos valores de $p_{i}$ utilizados, $p_{1}=p_{2}=p_{3}=0.4$. Na próxima figura apresentamos gráficos para a polarização da corrente nas três configurações magnéticas. 


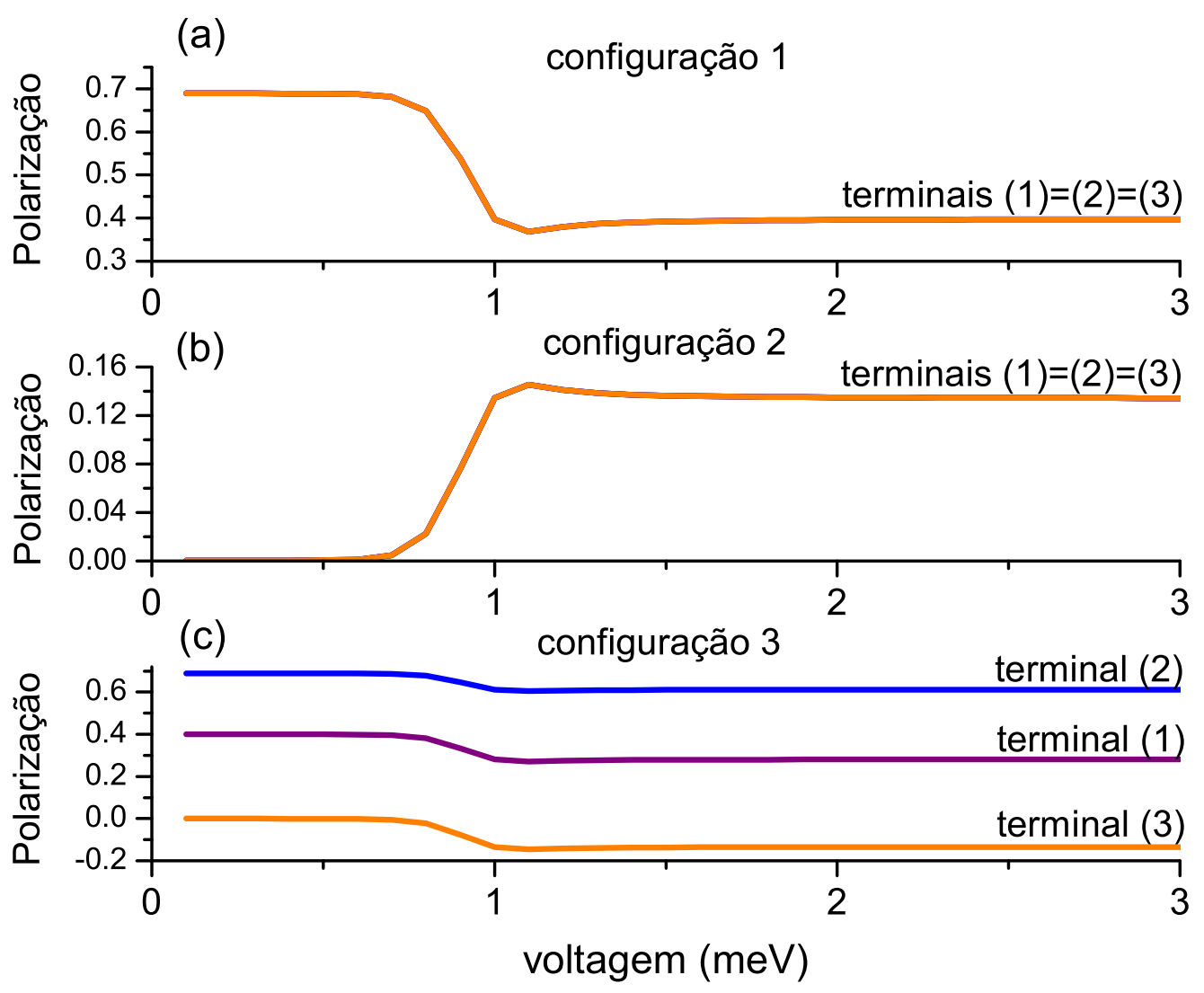

Figura 5.4: Polarização da corrente, $\wp=\left(I_{\uparrow}-I_{\downarrow}\right) /\left(I_{\uparrow}+I_{\downarrow}\right)$, nos vários terminais, como função da tensão externa aplicada. Na configuração 1 temos que as polarizações nos três terminais coindicem, sendo a polarização suprimida quando o sistema entra em ressonância $(e V \gtrsim 1 \mathrm{meV})$. Na configuração 2 temos uma amplificação da polarização quando há ressonância ( $\mathrm{V} \gtrsim 1 \mathrm{meV}$ ) no sistema. Nas configuração 1 e 2, as polarizações nos três terminais coincidem. Na configuração 3 observamos uma amplificação da polarização da corrente no terminal 2 em comparação com o terminal 1 (emissor). Já no terminal (3) temos não só uma supressão da polarização da corrente, mas também uma inversão do seu sinal. 

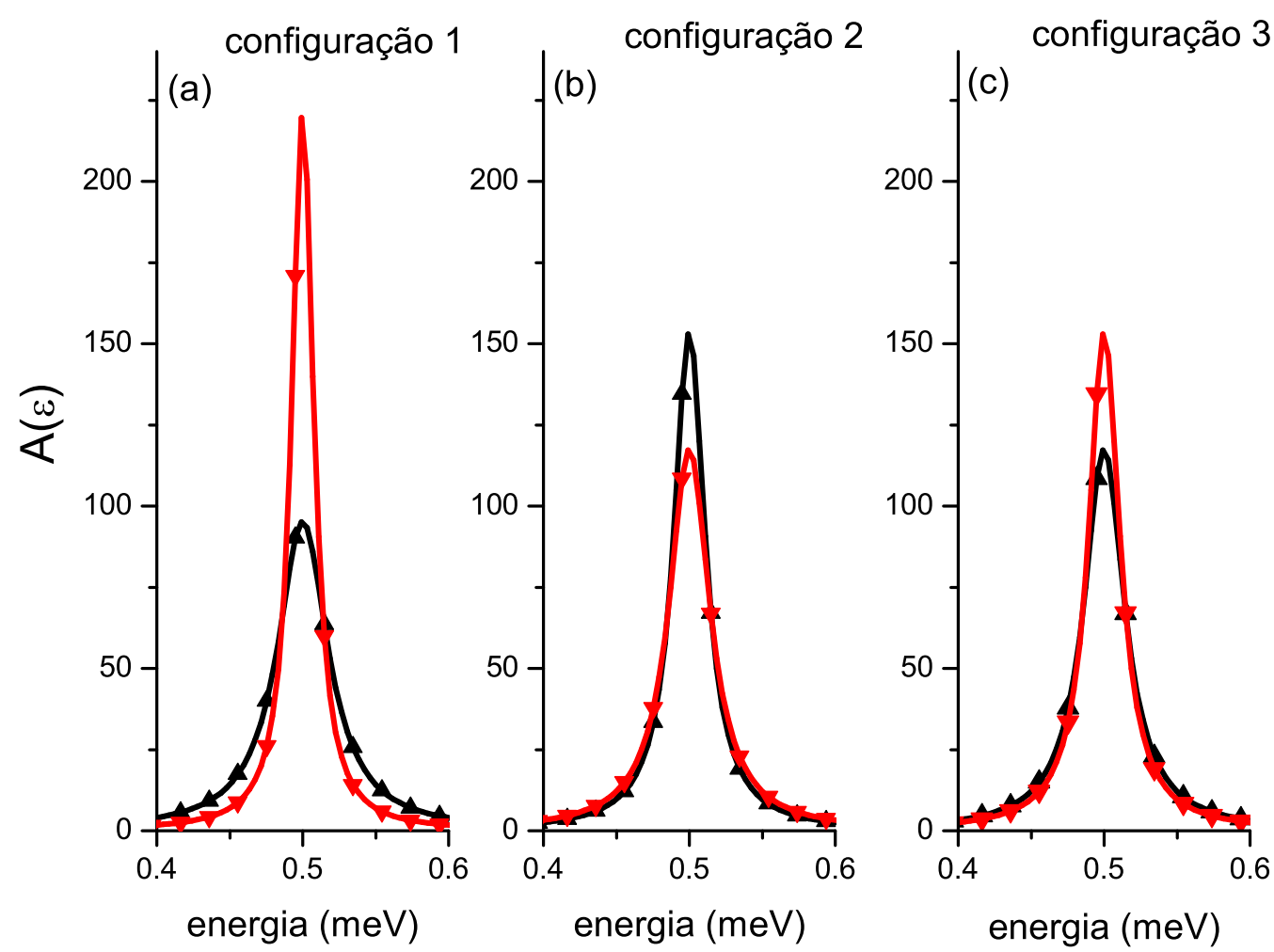

Figura 5.5: Funções espectrais $A_{\uparrow}(\epsilon)$ (linhas pretas) e $A_{\downarrow}(\epsilon)$ (linhas vermelhas) nas três configurações magnéticas dos terminais e $p_{1}=p_{2}=p_{3}$. Na configuração 1 temos $A_{\uparrow}$ mais largo do que $A_{\downarrow}$, porém de menor amplitude. Nas configurações 2 e $3, A_{\uparrow}$ e $A_{\downarrow}$ tem aproximadamente a mesma largura, sendo $A_{\uparrow}>A_{\downarrow}$ na configuração 2 e $A_{\uparrow}<A_{\downarrow}$ na configuração 3 . A forma dessas funções espectrais $A_{\uparrow}$ e $A_{\downarrow}$ [intensidades e larguras] nos auxiliam na compreensão dos resultados da Fig.(5.4). 
Magneto resistência de tunelamento (TMR). Para caracterizar o sistema anterior em termos de uma grandeza experimentalmente mais acessível, definimos abaixo TMRs adequadas, envolvendo as configurações 1, 2 e 3,

$$
T M R_{12}=\frac{I_{1}^{(1)}-I_{1}^{(2)}}{I_{1}^{(2)}}, \quad T M R_{13}=\frac{I_{1}^{(1)}-I_{1}^{(3)}}{I_{1}^{(3)}},
$$

onde os índices superiores indicam a configuração magnética do sistema (1, 2 ou 3), e o índice inferior o terminal. Note que utilizamos nessa definição somente a corrente no terminal 1 (emissor), e variamos a polarização do sistema. Definições equivalentes podem ser feitas utilizando os coletores, tendo em vista que a corrente se conserva no nosso sistema. A TMR $\mathrm{TM}_{12}$ corresponde a variação da resistência de tunelamento entre as configurações 1 e 2 , enquanto $\mathrm{TMR}_{13}$ nos dá a variação entre os alinhamentos 1 e 3 .

Na figura 5.6 mostramos curvas para a corrente total $I=I_{\uparrow}+I_{\downarrow}$ e a magneto resistência de tunelamento definidas acima, como função da tensão externa aplicada entre os eletrodos. Para tensões $\mathrm{eV}<1 \mathrm{meV}$ temos correntes nulas, visto que o nível do ponto quântico $\epsilon_{0}$ encontra-se acima do potencial químico do reservatório emissor. Quando $\mathrm{eV}=1 \mathrm{meV}$ o nível $\epsilon_{0}$ entra em ressonância e uma corrente começa a fluir pelo sistema.

Observamos que para $e V>1 \mathrm{meV}$, a corrente total é maior na configuração 1, em que todos os terminais estão alinhados paralelamente entre si (linha preta). Quando invertemos um dos terminais coletores com relação ao terminal emissor, a corrente diminui (linha azul). Quando os dois terminais coletores são alinhados antiparalelamente com relação ao emissor, a corrente diminui ainda mais (linha vermelha). Esse efeito é razoável, visto que ao passarmos da configuração 1 para a configuração 3, e depois para a configuração 2, temos um aumento sucessivo da resistência de tunelamento.

As curvas de TMR para cada configuração $\left[\mathrm{TMR}_{12}\right.$ e TMR $\left.\mathrm{TM}_{13}\right]$ apresentam dois plateaus. O primeiro plateau ocorre para tensões $\mathrm{eV}<1 \mathrm{meV}$, e o segundo para $e V>1 \mathrm{meV}$. O fato do TMR ser maior fora da ressonância $[e V<1 \mathrm{meV}]$ devese a diferença da largura de linha do nível $\epsilon_{0}$ entre as várias configuração. Por exemplo, o nível $\epsilon_{0}$ é mais largo na configuração 1 do que nas configurações $2 \mathrm{e}$ 3 [veja Fig. 5.5]. Com isso é natural que a corrente $I_{1}^{(1)}$ vá mais lentamente a zero do que $I_{1}^{(2)}$ e $I_{1}^{(3)}$ a medida em que o sistema sai de ressonância (isto é, ao passarmos por $e V=1 \mathrm{meV}$ partindo de tensões maiores). Como conseqüência disso, a diferença $I_{1}^{1}-I_{1}^{(2),(3)}$ aumenta fora da ressonância, e conseqüentemente o TMR cresce. Observamos ainda que os plateaus de TMR são maiores no caso $\mathrm{TMR}_{12}$ do que no caso $\mathrm{TMR}_{13}$. Isso ocorre porque a variação de resistência de tunelamento é maior entre as configurações 1 e 2 do que entre 1 e 3, o que está de acordo com as curvas de corrente [painel (a)].

Em suma, observamos que as variações nas TMRs acima são apreciáveis e 
distintas. Porém, não encontramos uma correlação direta entre as TMRs e as polarizações das correntes nos vários terminais. Estudos adicionais deverão ser desenvolvidos de modo a encontrar mecanismos de medida da polarização da corrente nesses eletrodos. Seguimos analisando a dependência das correntes $I_{\uparrow}$ e $I_{\downarrow}$ nos três terminais ferromagnéticos como função das polarizações $p_{1}, p_{2}$ e $p_{3}$.
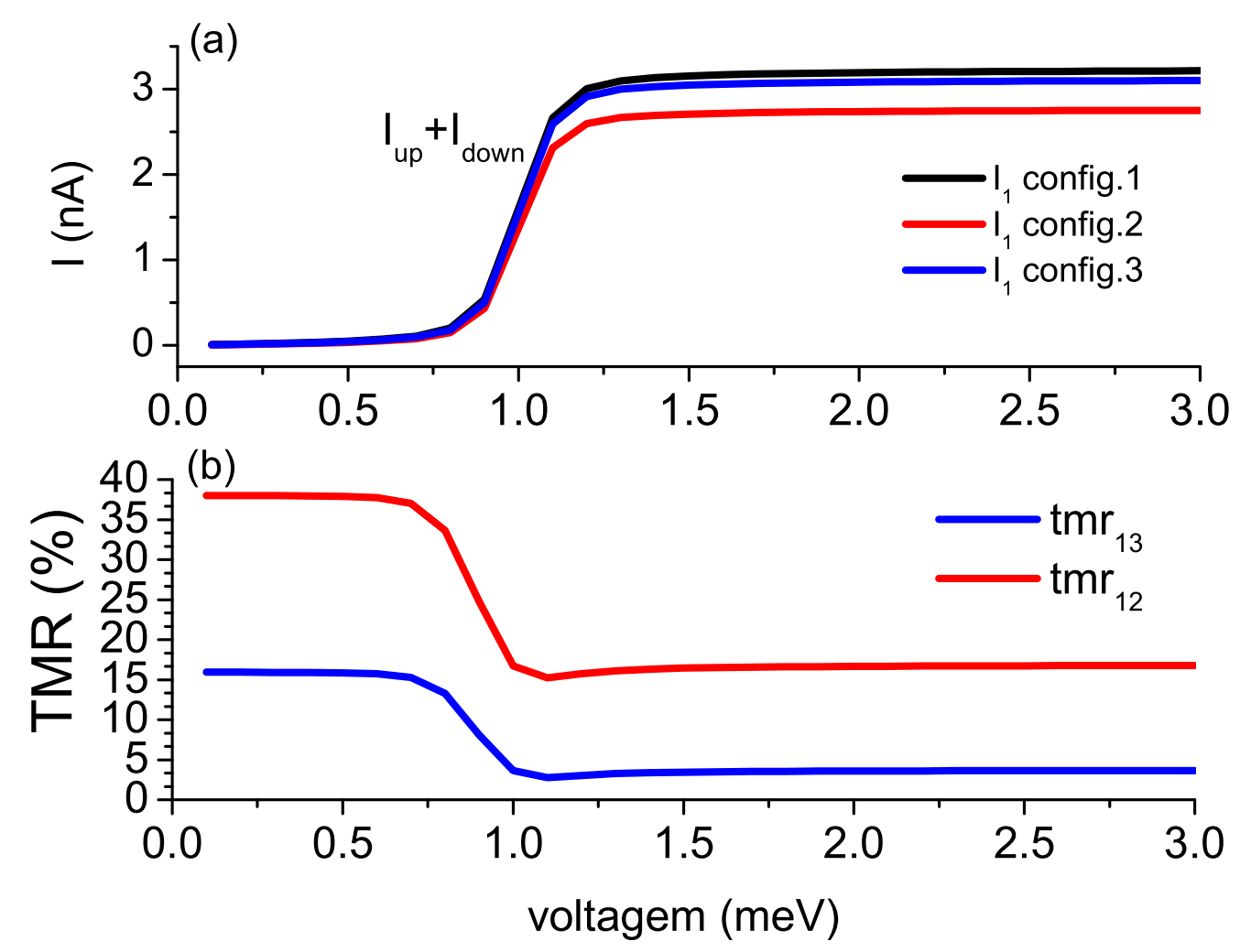

Figura 5.6: (c) Corrente total $\left(I_{\uparrow}+I_{\downarrow}\right)$, e (b) magneto resistência de tunelamento como função da voltagem. Observamos que a TMR é suprimido quando o sistema entra em ressonância $(e V=1 \mathrm{meV})$. Esse efeito deve-se a largura de linha do nível do ponto quântico $\epsilon_{0}$ nas várias configurações magnéticas. Os plateaus para $\mathrm{TMR}_{12}$ são maiores do que para $\mathrm{TMR}_{13}$. Isso se deve ao fato da variação da resistência de tunelamento ser mais apreciável entre os alinhamentos (1) e (2), do que entre (1) e (3).

\subsubsection{Dependência das correntes $I_{\uparrow}$ e $I_{\downarrow}$ como função da polarização dos eletrodos}

Nesta seção veremos como as correntes $I_{\uparrow}$ e $I_{\downarrow}$ variam com relação aos parâmetros $p_{1}, p_{2}$ e $p_{3}$, e como podemos otimizar a polarização das correntes como função desses parâmetros e do alinhamento ferromagnético dos terminais. 
Na figura (5.7) apresentamos gráficos para as corrente $I_{\uparrow}$ e $I_{\downarrow}$ no emissor, coletor (2) e coletor (3) [coluna da esquerda para a direita, respectivamente], em função de (a)-(c) $p_{1}$, (d)-(f) de $p_{2}$ ou (g)-(i) de $p_{3}$. Nos painéis em que variamos um determinado $p_{i}$, mantemos os demais fixos em 0.4. Por exemplo, nos painéis (a)(c) da Fig.(5.7) variamos $p_{1}$, e mantemos $p_{2}$ e $p_{3}$ constantes em 0.4. Observe que aumentando $p_{1}$ a corrente $I_{\uparrow}$ aumenta e a corrente $I_{\downarrow}$ diminui. Em particular, para $p_{1}=1$ temos $I_{\downarrow}=0 \mathrm{em}$ todos os terminais. Isso ocorre pois quando $p_{1}=1$, o terminal emissor fica $100 \%$ $\uparrow$-polarizado, deixando então de haver uma fonte de corrente $\downarrow$-polarizada no sistema. Enfatizamos aqui a ausência de espalhamento de spin no sistema. Veremos que quando $p_{2}=1$ ou $p_{3}=1$ (sendo $p_{1}<1$ ) a corrente $\downarrow$ não se anula em todos os terminais, como ocorre no caso $p_{1}=1$.

Ao variarmos $p_{2}$, mantendo $p_{1}$ e $p_{3}$ fixos [painéis (d)-(f)], vemos que a corrente $I_{\uparrow}$ aumenta no coletor (2), mas diminui no coletor (3). Em contraste, a corrente $I_{\downarrow}$ diminui, tendendo a zero no coletor (2), e aumenta no coletor (3). Podemos dizer que, com o aumento de $p_{2}$, os elétrons $\uparrow$ "preferem" entrar no terminal (2), em virtude do aumento do número de estados nesse terminal, enquanto os elétrons $\downarrow$ "preferem" ir para o terminal (3), devido a diminuição do número de estados $\downarrow$ no terminal (2). Em particular, para $p_{2}=1$ temos $I_{\downarrow}=0$ no coletor (2) e toda a corrente $I_{\downarrow}$ que sai do emissor entra no coletor (3).

Ao variarmos $p_{3}$ [painéis (g)-(i)] simplesmente "trocamos" o coletor (2) pelo coletor (3) e vice-versa. Ressaltamos que a corrente é sempre $\uparrow$-polarizada nesses painéis, isto é, não há mudança na polarização da corrente de um terminal a outro.

Já na configuração (2) [Fig.(5.8)] observamos que ao variarmos $p_{1}$ [painéis (a)(c)] , mantendo $p_{2}=p_{3}=0.4$, a corrente muda de polarização próxima a $p_{1} \approx 0.25$, sendo $\downarrow$-polarizada para $p_{1} \lesssim 0.25$ e $\uparrow$-polarizada para $p_{1} \gtrsim 0.25$ em todos os terminais. Note que mesmo para $p_{1}=0$ (emissor não polarizado) a corrente nesse terminal é $\downarrow$-polarizada $\left(I_{\downarrow}>I_{\uparrow}\right)$. Portanto, vemos que a polarização e o alinhamento magnético dos coletores afeta a polarização da corrente no terminal emissor, mesmo quando este não é ferromagnético.

Variando agora $p_{2}$ [Fig.5.8(d)-(f)] encontramos uma faixa de valores de $p_{2}$ [região achurada com linhas tracejadas] na qual a polarização da corrente no emissor e no coletor (2) trocam de sinal uma com relação a outra. Observe que dentro dessa faixa obtemos $I_{\uparrow}>I_{\downarrow}$ no emissor e $I_{\uparrow}<I_{\downarrow}$ no coletor (2). Já no coletor (3) [painel (f)] temos polarização $\uparrow$ para $0<p_{2}<1$, porém na região achurada do painel (f) a polarização da corrente $\left(I_{\uparrow}>I_{\downarrow}\right)$ é invertida com relação ao emissor $\left(I_{\uparrow}<I_{\downarrow}\right)$.

Nos painéis $(\mathrm{g})-(\mathrm{i})$ variamos $p_{3}$ mantendo $p_{1}=p_{2}=0.4$. Assim como nos painéis (d)-(e), aqui observamos uma faixa de valores para $p_{3}$ em que a corrente do emissor é $\uparrow$-polarizada e do coletor (3) é $\downarrow$-polarizada. Do mesmo modo temos uma faixa de valores na qual a polarização do emissor e do coletor (2) trocam de sinal um com relação ao outro. Note que na configuração (2), variar $p_{2}$ ou $p_{3}$ 
corresponde simplesmente a "trocar" terminal (2) por terminal (3) e vice-versa, assim como se dá na configuração (1).

Por último analisamos a configuração (3) na figura (5.9). Variando $p_{1}$ [painéis (a)-(c)] encontramos que a polarização da corrente nos terminais emissor e coletor (2) aumentam a medida que $p_{1}$ cresce, e para $p_{1} \rightarrow 1$ temos $I_{\downarrow}=0$, assim como ocorre nas configurações (1) e (2). Curiosamente, no coletor (3) temos que a corrente é $\downarrow$-polarizada para $p_{1} \lesssim 0.55$ [região achurada] e $\uparrow$-polarizada para $p_{1} \gtrsim$ 0.55. Portanto na região achurada temos uma inversão da polarização da corrente com relação ao emissor.

Variando $p_{2}$ [painéis (d)-(f)] vemos que as polarizações no emissor e no coletor (2) intensificam-se com o aumento de $p_{2}$, enquanto que no terminal (3) a polarização torna-se mais negativa com o aumento de $p_{2}$. Para toda a faixa de valores de $p_{2}$ a corrente no coletor (3) é $\downarrow$-polarizada, portanto temos uma polarização contrária a dos demais terminais.

Nos painéis (g)-(i) variamos $p_{3}$. Encontramos que com o aumento de $p_{3}$ a corrente no emissor torna-se menos polarizada $\left(I_{\uparrow}\right.$ se aproxima de $\left.I_{\downarrow}\right)$. Ao contrário, a polarização no terminal (2) aumenta, e no terminal (3) muda de sinal a partir de $p_{3} \approx 0.2 \mathrm{meV}$ [região achurada]. Na região achurada dos painéis (c) e (i) temos que a polarização no coletor (3) é negativa $\left(I_{\downarrow}>I_{\uparrow}\right)$, em oposição a dos demais terminais.

Os painéis apresentados aqui nos revelam a possibilidade de se gerar em um único dispositivo correntes com polarizações $\uparrow$ e $\downarrow$ simultaneamente. Sistemas que gerem correntes com duas polarizações ao mesmo tempo poderão eventualmente encontrar aplicações dentro da eletrônica de spin. 


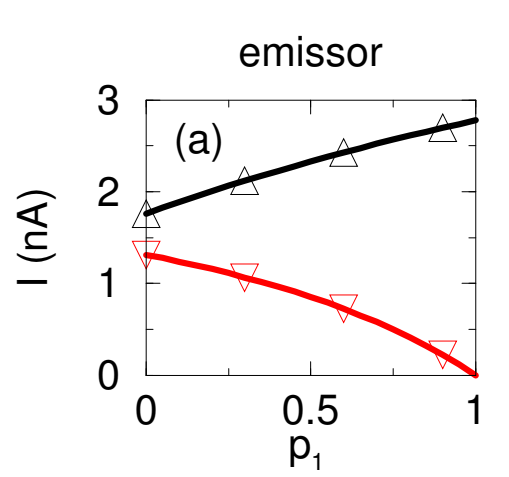

\section{Configuracao 1}

\section{coletor (2)}
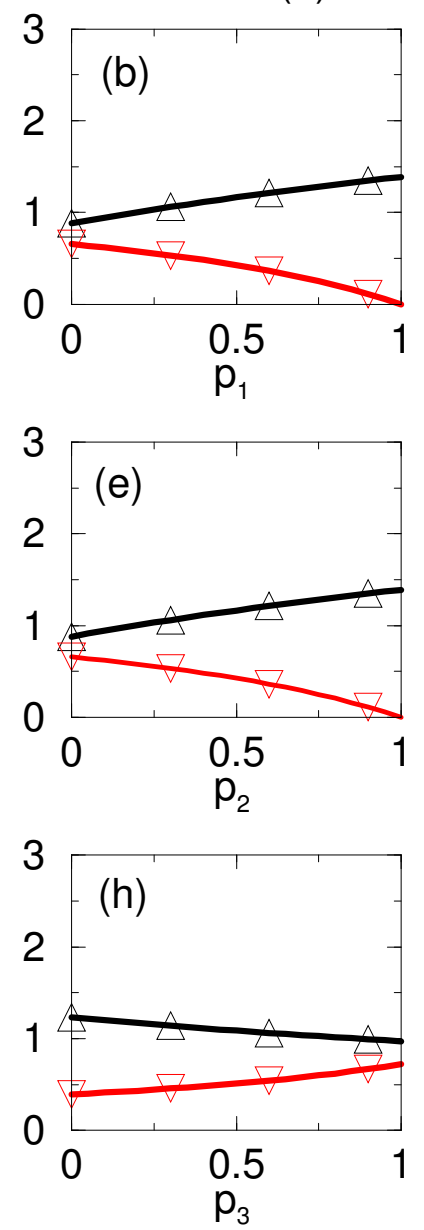

coletor (3)
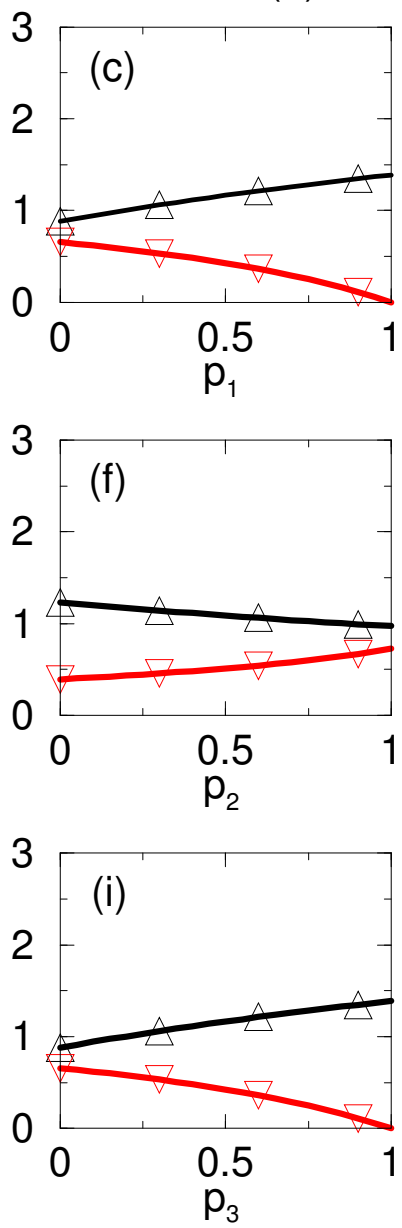

Figura 5.7: Correntes $I_{\uparrow}$ e $I_{\downarrow}$ como função de $p_{i}$, sendo $i=1$ na primeira linha de painéis [(a)-(c)], $i=2$ na segunda linha $[(\mathrm{d})-(\mathrm{f})]$ e $i=3$ na terceira linha [(g)-(i)]. Na linha em que variamos um determinado $p_{i}$, mantemos os demais constantes em 0.4. Ao aumentarmos $p_{1}$ [(a)-(c)] a diferença entre as correntes $I_{\uparrow}$ e $I_{\downarrow}$ aumenta, e portanto a polarização das correntes também aumenta. Variando $p_{2}$, a polarização da corrente aumenta no emissor e coletor (2), mas diminui no coletor (3). Os painéis (g)-(i) (onde variamos $p_{3}$ ) são idênticos aos painéis (d)-(f) trocando-se (f) por (h) e (e) por (i). Tensão externa adotada: $\mathrm{eV}=2 \mathrm{meV}$. 


\section{Configuracao 2}

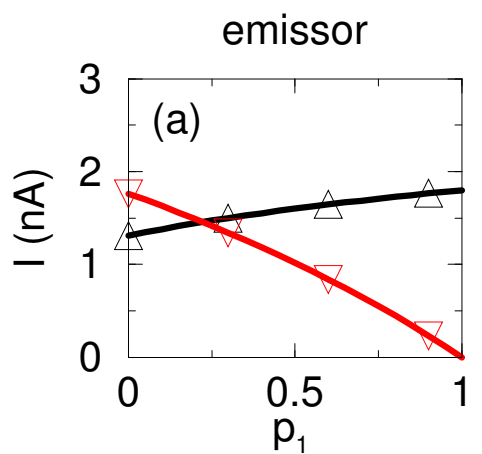

coletor (2)
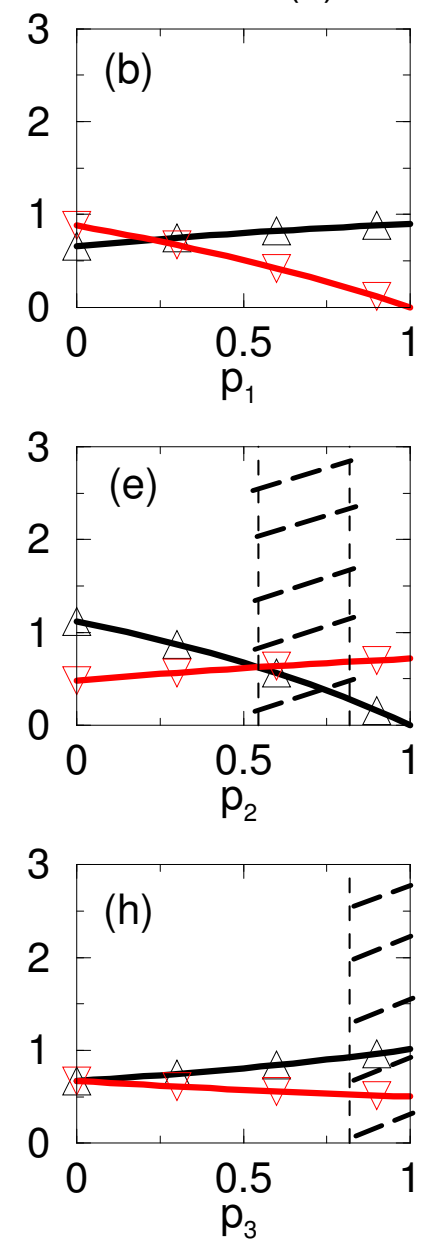

coletor (3)
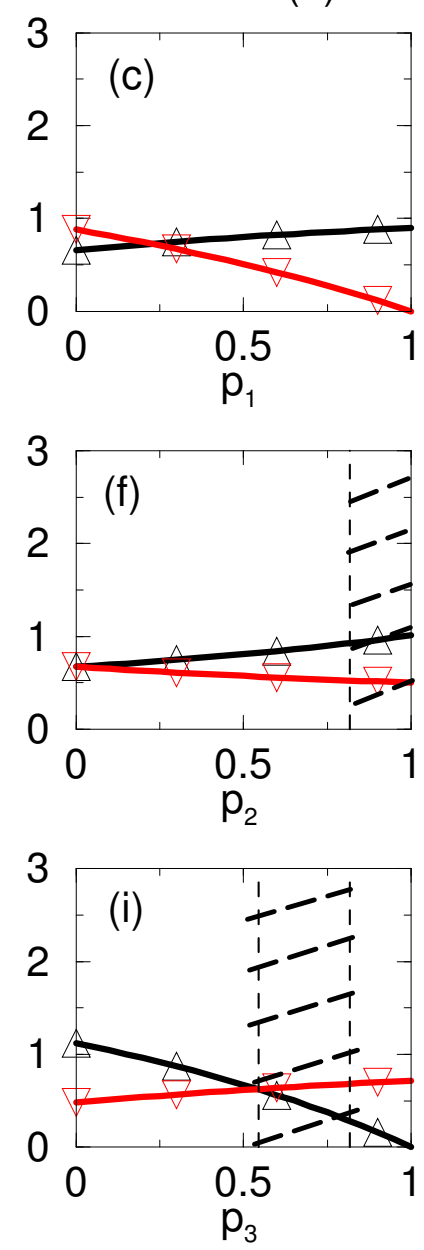

Figura 5.8: Curvas análogas as da Fig.(5.7), exceto pela configuração magnética dos terminais (configuração 2). Nesse caso ao variarmos $p_{1}$ (fixando $p_{2}=p_{3}=0.4$ ) observamos que há uma inversão na polarização da corrente em torno de $p_{1} \approx 0.25$. Variando $p_{2}$ ou $p_{3}$ encontramos faixas de valores [indicadas pelas regiões achuradas] em que as polarizações das correntes mudam de sinal de um terminal a outro. Por exemplo, comparando os painéis (d) e (e). Vemos que há uma faixa de $p_{2}$ na qual a corrente é $\uparrow$-polarizada no emissor e $\downarrow$-polarizada no coletor (2). Uma inversão da polarização da corrente também se dá entre emissor e coletor (3) conforme vemos comparando painéis (d) e (f). Os painéis (g)-(i) também mostram inversões nas polarizações das correntes. 


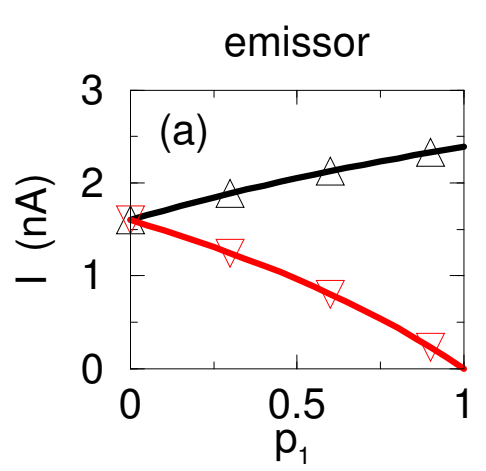

\section{Configuracao 3}

coletor (2)
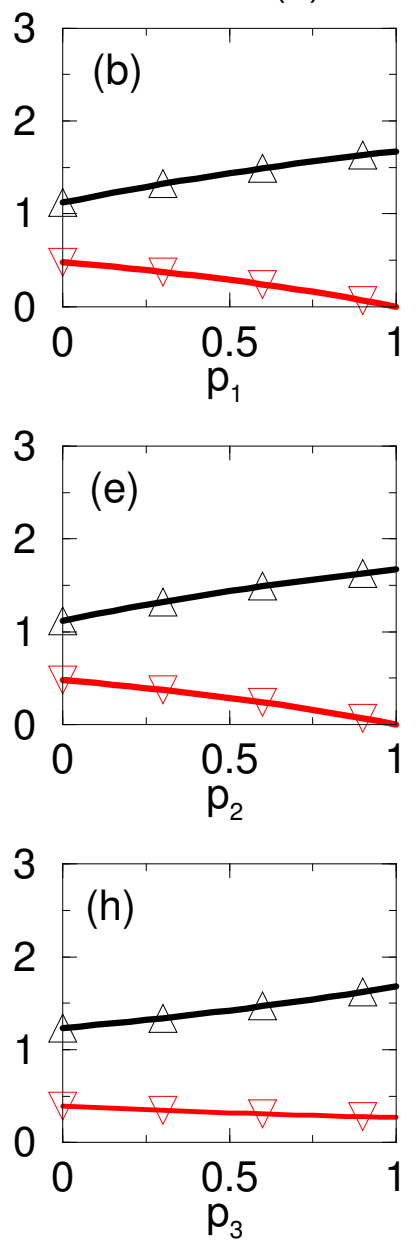

coletor (3)
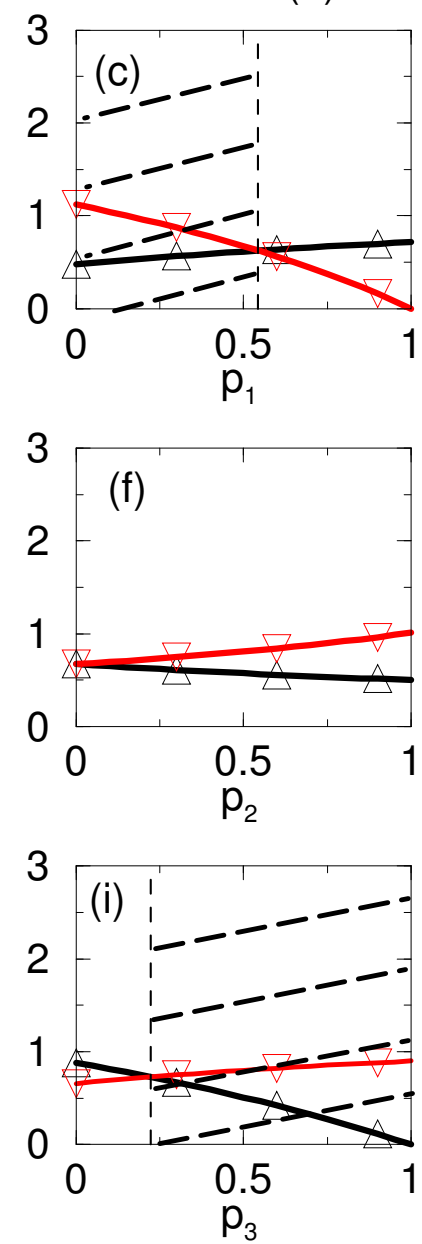

Figura 5.9: Curvas análogas as da figura anterior, exceto pelo alinhamento magnético dos terminais (configuração 3). Nessa configuração observamos cruzamentos das correntes $I_{\uparrow}$ e $I_{\downarrow}$ somente no coletor (3). Temos uma faixa de valores de $p_{1}$ [painel (c)] e de $p_{3}$ [painel (i)] em que a corrente do coletor (3) apresenta polarização contrária a do emissor e do coletor (2), isto é, $I_{\uparrow}<I_{\downarrow}$ [veja regiões achuradas]. Note que para $p_{1}=p_{3}=0.4$ e $p_{2}$ qualquer [painel (f)] temos sempre uma corrente $\downarrow$-polarizada no coletor (3). Além disso pode-se variar $p_{1}$ ou $p_{3}$ de modo a se obter uma corrente $\uparrow$-polarizada no terminal (3). Nos demais terminais temos sempre uma corrente $\uparrow$-polarizada. 


\subsection{Associação de resistores}

Aqui propomos uma associação de resistores que reproduz qualitativamente alguns resultados do sistema estudado nesse capítulo: três terminais ferromagnéticos acoplados a um ponto quântico [Fig.(5.10)]. Essa associação de resistores nos permite explicar de maneira simples e intuitiva alguns resultados obtidos para a corrente dependente de spin, por exemplo as Figs.(5.7)-(5.9).

Considere a associação de resistores ilustrada na Fig.(5.10). O ramo superior corresponde a um canal para spin $\uparrow$, enquanto o ramo inferior a um canal para spin $\downarrow$. Cada resistência equivale a uma barreira de tunelamento "vista" pelos elétrons ao entrarem e saírem do ponto quântico. Nesse esquema de resistores o ponto quântico (PQ) estaria situado na posição indicada pelas bolas vermelhas. Definimos

$$
r_{i}^{\sigma}=\frac{\hbar}{e^{2}} \frac{\Gamma_{0}}{\Gamma_{\sigma}^{(i)}},
$$

onde o parâmetro $\Gamma_{\sigma}^{(i)}$ é dado pelas Eqs.(5.31)-(5.33). Após o "ponto quântico" cada um dos segmentos para spin $\uparrow \mathrm{e} \downarrow$ se desdobra em dois ramos, formando uma associação de resistores em paralelo. Para os spins $\uparrow$ temos $r_{2}^{\uparrow}$ e $r_{3}^{\uparrow}$ em paralelo, e para os spins $\downarrow$ temos $r_{2}^{\downarrow}$ e $r_{3}^{\downarrow}$ em paralelo. Na presença de uma tensão externa $V$ entre os extremos (pontos A e B), surge uma corrente no resistor $r_{1}^{\sigma}$ dada por

$$
I_{1}^{\sigma}=\frac{1}{r_{1}^{\sigma}+r_{23}^{\sigma}} V
$$

onde $\frac{1}{r_{23}^{\sigma}}=\frac{1}{r_{2}^{\sigma}}+\frac{1}{r_{3}^{\sigma}}$. As correntes nos resistores $r_{2}^{\sigma}$ e $r_{3}^{\sigma}$ são dadas por

$$
I_{2}^{\sigma}=\frac{r_{23}^{\sigma}}{r_{2}^{\sigma}\left(r_{1}^{\sigma}+r_{23}^{\sigma}\right)} V
$$

$\mathrm{e}$

$$
I_{3}^{\sigma}=\frac{r_{23}^{\sigma}}{r_{3}^{\sigma}\left(r_{1}^{\sigma}+r_{23}^{\sigma}\right)} V
$$

respectivamente.

Com as expressões das correntes, geramos curvas para $I_{i}^{\sigma}$ versus $p_{1}, p_{2}$ e $p_{3}$. Interessantemente encontramos resultados idênticos aos das Figs.(5.7)-(5.9). Isso então nos permite entender as variações das correntes como função de $p_{i}(i=$ 1,2,3), observada nas Figs.(5.7)-(5.9), em termos das "resistências" sentidas pelos elétrons ao tunelar através do sistema. Por exemplo, na configuração 3, quando $p_{3} \rightarrow 1$, mantendo $p_{1}=p_{2}=0.4$, encontramos que a corrente $I_{2}^{\uparrow}$ aumenta enquanto $I_{3}^{\uparrow}$ vai a zero [veja painéis (h) e (i) da Fig.(5.9)]. Em termos da associação de resistores, entendemos esse fato notando que para $p_{3}=1$ e configuração 3 temos $\Gamma_{\uparrow}^{(3)}=0$ e $\Gamma_{\downarrow}^{(3)}=2 \Gamma_{0}$, o que equivale a $r_{3}^{\uparrow} \rightarrow \infty$. Portanto nenhuma corrente $\uparrow$ passará pelo resistor $r_{3}^{\uparrow}$ da Fig.(5.10) [ou equivalentemente pelo coletor 3], e toda a corrente $\uparrow$ que passar pelo resistor $r_{1}^{\uparrow}$ também passará pelo resistor $r_{2}^{\uparrow}$. Do mesmo modo, com o aumento de $p_{3}$ a resistência $r_{3}^{\downarrow}$ diminui ligeiramente, resultando no 
acréscimo da corrente $I_{3}^{\downarrow}$ observado na Fig.5.9(h). Análises semelhantes se aplicam para as demais configurações magnéticas estudadas e diferentes valores de $p_{i}$.

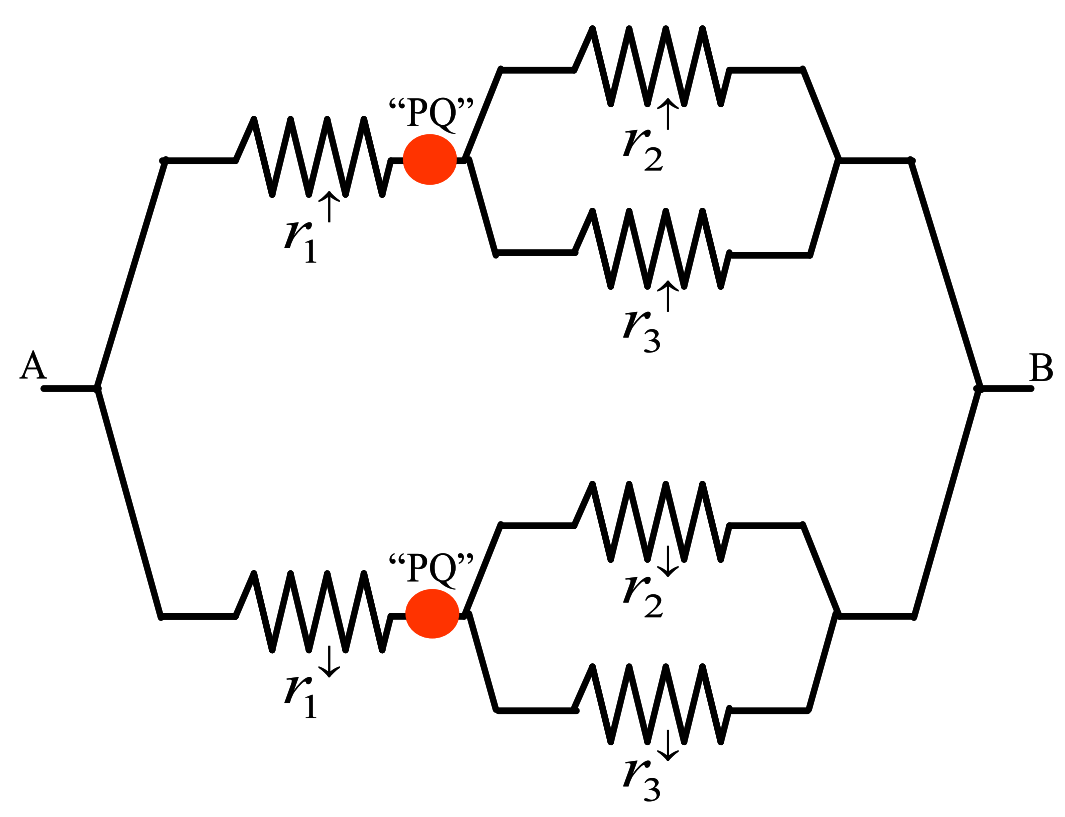

Figura 5.10: Associação de resistores que simula o sistema de um ponto quântico acoplado a três terminais ferromagnéticos. O ramo superior dessa associação corresponde a um canal para spins $\uparrow$, enquanto o ramo inferior a um canal para spins $\downarrow$. As bolas vermelhas indicam a posição correspondente ao ponto quântico nesse sistema. Os resistores em paralelo $r_{2}^{\sigma}$ e $r_{3}^{\sigma}$ após o "ponto quântico" equivale aos coletores 2 e 3 , respectivamente.

Em suma, estudamos corrente spin-polarizada em um sistema constituído por um ponto quântico acoplado a três terminais ferromagnéticos. Observamos que a corrente resolvida em spin depende fortemente das configurações magnéticas dos eletrodos. Por exemplo, dependendo da polarização de cada eletrodo e dos seus respectivos alinhamentos, podemos ter uma corrente saindo $\uparrow$-polarizada do terminal emissor, e entrando $\downarrow$-polarizada em um terminal coletor [veja, por exemplo, as Figs.(5.8) e (5.9)]. Além disso pode haver um ganho na polarização da corrente ao passar de um terminal a outro. 


\section{Capítulo 6}

\section{Poço quântico acoplado a contatos semimagnéticos}

Nesse capítulo desenvolvemos equações de transporte (corrente e ruído) para o sistema constituído de um poço quântico acoplado, via barreiras de tunelamento, a terminais semicondutores magnéticos diluído $(\mathrm{DMS})^{1}$. Obtivemos equações para a corrente e para o ruído, em termos de funções de Green de não equilíbrio, que levam em conta a interação de troca $s$ - $d$ nos contatos magnéticos e quantização de Landau devido a um campo magnético externo. O sistema analisado aqui difere em alguns aspectos da junção ferromagneto-ponto-quântico-ferromagneto discutida no capítulo 4. Por exemplo, no sistema DMS utilizamos um campo magnético para polarizar os terminais via interação de troca $s$ - $d$. Portanto os efeitos dependentes de spin surgem somente na presença de um campo magnético externo, em contraste com o sistema FM-PQ-FM, onde temos efeitos dependentes de spin mesmo à $B=0^{2}$. Uma outra diferença está na densidade de estados do poço e do ponto quântico. Enquanto no ponto quântico temos um único estado degenerado para spins $\uparrow$ e $\downarrow$, no poço há uma infinidade de estados que podem conduzir corrente. Essa corrente e o seu ruído serão graficados no decorrer do capítulo. Em particular, analisaremos corrente e ruído como função da tensão externa e do campo magnético aplicado. Alguns dos efeitos observados foram a forte dependência da polarização da corrente como função da tensão externa, e oscilações na corrente, no ruído e no fator de Fano como função do campo magnético. Também observamos como a tensão externa afeta a largura de linha dos níveis de Landau do poço.

\footnotetext{
${ }^{1}$ Recentemente Slobodskyy et al. [62] desenvolveram um diodo de tunelamento ressonante baseado em DMS (ZnMnSe). Também J. C. Egues et al. [61] calcularam magnetoresistência em sistemas de uma e de duas barreiras com terminais e/ou poço DMS.

${ }^{2}$ Notamos que no sistema FM-PQ-FM descrito anteriormente, um campo magnético externo seria utilizado apenas para passar da configuração P para AP.
} 


\subsection{Propriedades magnéticas e a interação de troca}

Os materiais DMS são semicondutores com uma baixa concentração de impurezas magnéticas [89],[90]. Um exemplo de material DMS é a liga ternária do grupo II-VI $Z_{1-x} \mathrm{Mn}_{x}$ Se. O manganês tem um spin permanente $S=5 / 2$, proveniente do orbital semi-preenchido $3 d^{5}$, sendo a configuração eletrônica do Mn dada por [Ar] $3 d^{5} 4 s^{2}$. Como para adicionar um elétron nesse orbital custa demasiada energia (6 a $7 \mathrm{eV}$ ), temos que os átomos de Mn são inertes, assemelhando-se aos átomos de Zn ([Ar] $\left.3 d^{10} 4 s^{2}\right)$. Sendo assim, na liga ternária o Mn ocupa sítios de Zn, resultando numa sub-rede com alguns sítios magnéticos.

Considerando um arranjo paramagnético de ions de Mn, temos que o spin médio $\left\langle S_{z}\right\rangle$ de um átomo de Mn é dado pela seguinte média

$$
\left\langle S_{z}\right\rangle=\frac{\sum_{S_{z}=-S}^{S} S_{z} e^{-\frac{g_{M n} \mu_{B} B S_{z}}{k_{B} T}}}{\sum_{S_{z}=-S}^{S} e^{-\frac{g_{M n} \mu_{B} B S_{z}}{k_{B} T}}}=-S B_{S}(\eta),
$$

onde $B_{S}$ é a função de Brillouin de spin $S$, e $\eta=\frac{g_{M n} \mu_{B} B}{k_{B} T}$ é a razão entre a energia magnética e a energia térmica, sendo $g_{M n}$ o fator giromagnético, $\mu_{B}$ o magneton de Bohr, $k_{B}$ a constante de Boltzmann, e $T$ a temperatura ${ }^{3}$. O campo magnético $B$ é tomado na direção $\hat{z}$, que corresponde a direção perpendicular ao poço quântico. A função de Brillouin tem a seguinte forma

$$
B_{S}(\eta)=\frac{1}{S}\left\{\left(S+\frac{1}{2}\right) \operatorname{coth}\left[\left(S+\frac{1}{2}\right) \eta\right]-\frac{1}{2} \operatorname{coth} \frac{\eta}{2}\right\}
$$

A figura (6.1) ilustra o comportamento de $\left\langle S_{z}\right\rangle$ como função do campo $B$ para $S=5 / 2$ e diferentes valores de $T$. Observamos que, quanto menor a temperatura, mais rápido $\left\langle S_{z}\right\rangle$ satura em $-5 / 2$. Isso ocorre porque quanto menor $T$, menor é a agitação térmica que tende a desalinhar os spins da direção preferencial imposta pelo campo magnético externo.

Interação de troca. Nos semicondutores magnéticos diluídos os elétrons da banda de condução interagem com os elétrons localizados do orbital $d$ do $\mathrm{Mn}$. Essa interação recebe o nome de interação de troca $s$ - $d$, e pode ser descrita através do Hamiltoniano de Heisenberg [89]

$$
H_{s d}=-\sum_{\mathbf{R}_{j}} J\left(\mathbf{r}-\mathbf{R}_{j}\right) \mathbf{S}_{j} \cdot \sigma
$$

onde $J\left(\mathbf{r}-\mathbf{R}_{j}\right)$ é a constante de troca entre um elétron de condução na posição $\mathbf{r}$ e um elétron localizado no orbital $d$ do $j$-ésimo sítio de impureza Mn. Temos que $\sigma$ e $\mathbf{S}_{j}$ correspondem aos operadores de spin dos elétrons de condução e da $j$-ésima impureza, respectivamente.

\footnotetext{
${ }^{3}$ Não consideramos temperaturas efetivas.
} 


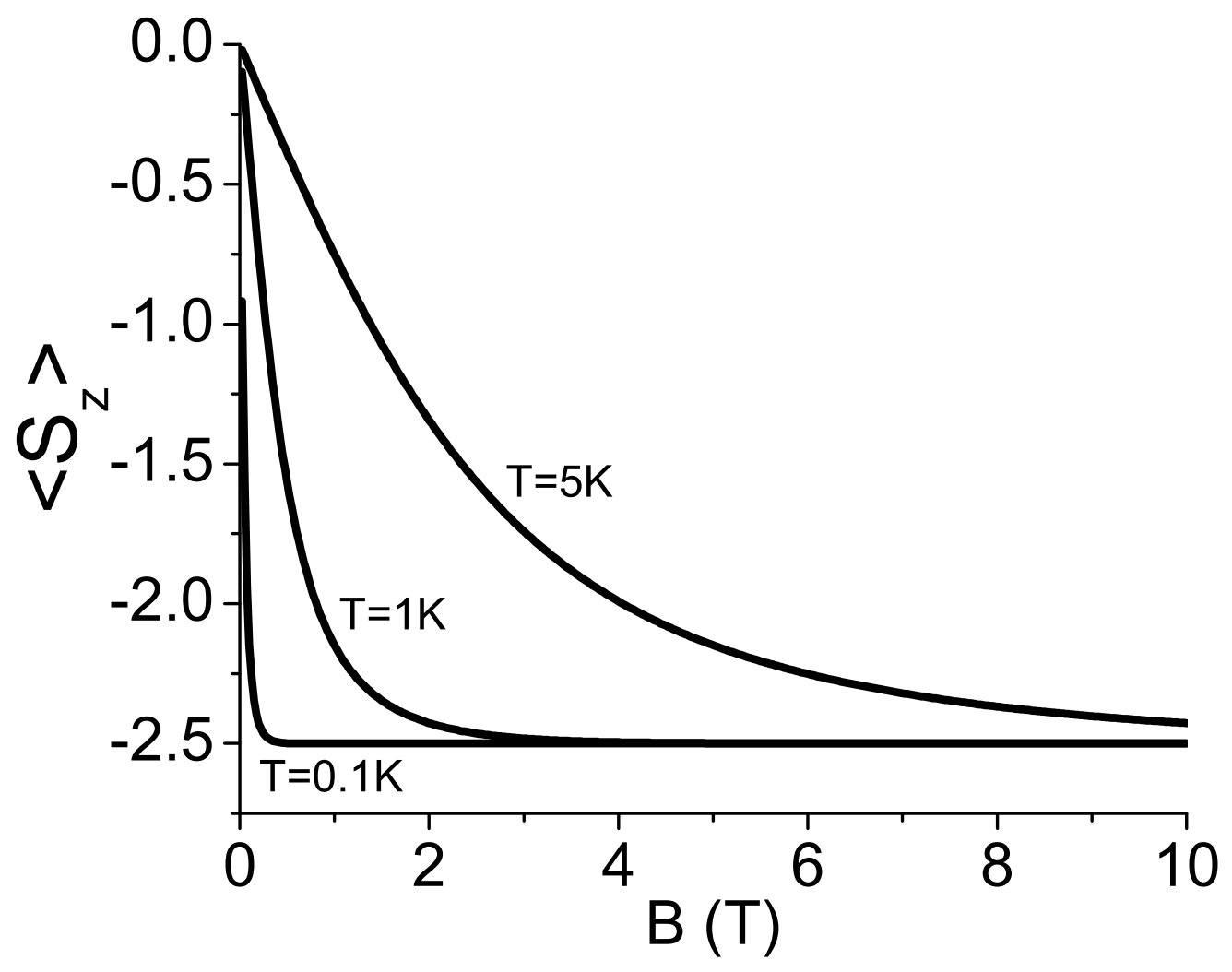

Figura 6.1: Spin médio das impurezas de Mn na liga ternária de ZnMnSe como função do campo magnético. A função $\left\langle S_{z}\right\rangle$ segue o comportamento da função de Brillouin $B_{5 / 2}$, que descreve os spins paramagnéticos do $\mathrm{Mn}(\mathrm{S}=5 / 2)$. Diferentes temperaturas são ilustradas.

O Hamiltoniano (6.3) não possui invariância translacional, visto que as impurezas ocupam sítios $\mathbf{R}_{j}$ que estão distribuídos aleatoriamente. Para restabelecer essa invariância utiliza-se a aproximação do cristal virtual, que abreviaremos por $\mathrm{VCA}^{4}$. Essa aproximação consiste em atribuir a cada sítio da sub-rede de cátions uma fração $x J(\mathbf{r}-\mathbf{R})$ para o potencial de troca. Com isso a distribuição de impurezas passa a ser periódica. Porém, a orientação de cada spin das impurezas não segue necessariamente uma periodicidade. Para contornar esse problema utiliza-se a aproximação de campo médio: como os elétrons estendidos "vêem" várias impurezas, substitui-se o spin $\mathbf{S}_{j}$ de cada uma por uma média térmica $\langle\mathbf{S}\rangle$. Na presença de um campo magnético $\mathbf{B}=B \widehat{z}$ temos que $\left\langle\mathbf{S}_{j}\right\rangle=\left\langle S_{z}\right\rangle \widehat{z}$, e $\left\langle S_{x}\right\rangle=\left\langle S_{y}\right\rangle=0$. Com essas duas aproximações, o Hamiltoniano de troca (6.3) assume a seguinte forma

$$
H_{s d}^{M F}=-x\left\langle S_{z}\right\rangle \sigma_{z} \sum_{\mathbf{R}} J(\mathbf{r}-\mathbf{R})=-x\left\langle S_{z}\right\rangle \sigma_{z} N_{0} \alpha
$$

\footnotetext{
${ }^{4} \mathrm{VCA}=$ virtual cristal approximation
} 
onde o vetor $\mathbf{R}$ indica a posição de cada sítio da sub-rede de cátions, $\sigma_{z}= \pm 1 / 2, N_{0}$ é a densidade de cátions, e $x$ a densidade de impurezas de Mn. Para obter o último termo da Eq.(6.4) usamos a aproximação da massa efetiva e função envelope. O fator $N_{0} \alpha$ é conhecido para diversos materiais, por exemplo, $N_{0} \alpha=0.26 \mathrm{eV}$ para elétrons no $\mathrm{Zn}_{1-x} \mathrm{Mn}_{x}$ Se [90].

A interação $s$ - $d$ dá origem ao efeito Zeeman gigante na presença de um campo magnético externo, o que leva a um desdobramento das bandas de energia para spins $\uparrow$ e $\downarrow$. Esse desdobramento de bandas resulta em efeitos dependentes de spin. Vale notar que no contexto de heteroestruturas, materiais com bandgaps dependentes de spin, quando colocados em camadas diferentes, geram potenciais dependentes de spin [91].

\subsection{Modelo}

Modelamos o sistema constituído de dois terminais DMS acoplados a um poço quântico através do seguinte Hamiltoniano [94]

$$
H=\sum_{\beta \eta} \epsilon_{\beta \eta} c_{\beta \eta}^{\dagger} c_{\beta \eta}+\sum_{\alpha} \epsilon_{\alpha} d_{\alpha}^{\dagger} d_{\alpha}+\sum_{\alpha \beta \eta}\left(V_{\alpha \beta} c_{\beta \eta}^{\dagger} d_{\alpha}+V_{\alpha \beta}^{*} d_{\alpha}^{\dagger} c_{\beta \eta}\right)
$$

onde o operador $c_{\beta \eta}^{\dagger}\left(c_{\beta \eta}\right)$ cria (destrói) um elétron no terminal $\eta$. Analogamente temos $d_{\alpha}^{\dagger}\left(d_{\alpha}\right)$ para o poço quântico. Os índices $\alpha$ e $\beta$ são conjuntos de números quânticos sendo $\alpha$ para o poço e $\beta$ para os terminais: $\alpha=\left(k_{y}, n, \sigma\right)$, $\beta=\left(k_{y \eta}, k_{z \eta}, m, \sigma\right)$. Temos que $n$ e $m$ são os índices dos níveis de Landau que surgem na presença do campo magnético $\mathbf{B}=B \hat{z}, \sigma$ e $\sigma^{\prime}$ são índices de spin, $k_{y}$ e $k_{y \eta}$ são vetores de onda na direção $\hat{y}$ e $k_{z \eta}$ é um vetor de onda na direção $\hat{z}$. As somas em $\alpha$ e $\beta$ equivalem a somar sobre todos os números quânticos. O terceiro termo da Eq.(6.5) é o termo de tunelamento que acopla os reservatórios ao poço quântico, sendo $V_{\alpha \beta}$ o parâmetro de acoplamento.

A energia $\epsilon_{\beta \eta}$ dos elétrons no reservatório $\eta$ tem a seguinte forma

$$
\epsilon_{\beta \eta}=\frac{\hbar^{2} k_{z \eta}^{2}}{2 m^{*}}+\hbar \omega_{c}\left(m+\frac{1}{2}\right)+E_{s d}+\xi_{\eta} e V
$$

onde $\omega_{c}=\frac{e B}{m^{*}}$ e $m^{*}$ é a massa efetiva do elétron, e $E_{s d}=-x N_{0} \alpha\left\langle S_{z}\right\rangle \sigma_{z}$. Para tornar essa equação plausível notemos o seguinte [92]: visto que não existem componente da força de Lorentz na direção do campo (direção $\hat{z}$ ), a energia cinética nessa direção não é afetada pelo campo, e portanto continua valendo $\hbar^{2} k_{z}^{2} / 2 m^{*}$. Porém, a energia de movimento perpendicular ao campo, que seria $\hbar^{2}\left(k_{x}^{2}+k_{y}^{2}\right) / 2 m^{*}$ na ausência de campo, é quantizada em $\hbar \omega_{c}$, dando origem ao termo $\hbar \omega_{c}\left(m+\frac{1}{2}\right)$. Esse fenômeno recebe o nome de quantização orbital. Os vários níveis $m$ são os já mencionados níveis de Landau. O terceiro termo na Eq.(6.6) corresponde a interação de troca $s$-d, e o quarto leva em conta a tensão externa $V$. O fator 
$\xi_{\eta}$ é definido tal que $\xi_{L}=0$ e $\xi_{R}=-1$. Com isso temos que o terminal da esquerda funciona como emissor e o da direita como coletor de elétrons. Observe que a energia (6.6) é degenerada em $k_{y \eta}$. Essa degenerescência recebe o nome de degenerescência de Landau $\left(g_{B}=L_{x} L_{y} e B / h\right)$, e se deve ao fato das órbitas dos elétrons no plano $x y$ poderem estar centradas em qualquer ponto desse plano. Por isso essa degenerescência é proporcional a seção transversal do sistema $L_{x} L_{y}$.

Para os elétrons no poço quântico temos a seguinte energia

$$
\epsilon_{\alpha}=\epsilon_{0}+\hbar \omega_{c}\left(n+\frac{1}{2}\right)+\xi_{p q} e V .
$$

O primeiro termo $\epsilon_{0}$ é uma constante que define o mínimo de energia. O segundo termo fornece os níveis de Landau $n=0,1,2, \ldots$, e o terceiro leva em conta a tensão externa. Para o fator $\xi_{p q}$ adotamos $\xi_{p q}=-1 / 2$, o que corresponde a uma queda de tensão de $-e V / 2$ entre emissor e poço quântico. Observe que $\epsilon_{\alpha}$ também é degenerado em $k_{y}$. Com o Hamiltoniano (6.5) e as energias (6.6) e (6.7) seguimos para o cálculo das expressões da corrente e do ruído.

\subsection{Corrente}

Deduzimos a equação da corrente seguindo o mesmo procedimento dos capítulos anteriores. A partir da definição $I_{\eta}=-e\left\langle\dot{N}_{\eta}\right\rangle=-\frac{i e}{\hbar}\left\langle\left[H, N_{\eta}\right]\right\rangle$, onde $N_{\eta}=$ $\sum_{\beta} c_{\beta \eta}^{\dagger} c_{\beta \eta}$ é o número total de elétrons no reservatório $\eta$, obtemos

$$
I_{\eta}=\frac{i e}{\hbar} \sum_{\alpha \beta} V_{\alpha \beta}\left\langle c_{\beta \eta}^{\dagger}(t) d_{\alpha}(t)\right\rangle-\frac{i e}{\hbar} \sum_{\alpha \beta} V_{\alpha \beta}^{*}\left\langle d_{\alpha}^{\dagger}(t) c_{\beta \eta}(t)\right\rangle,
$$

onde o Hamiltoniano utilizado no comutador $\left[H, N_{\eta}\right]$ está dado na Eq.(6.5). Definindo a função de Green menor $G_{\alpha \beta \eta}^{<}(t, t)=i\left\langle c_{\beta \eta}^{\dagger}(t) d_{\alpha}(t)\right\rangle$, podemos reescrever a corrente como segue

$$
I_{\eta}=\frac{2 e}{\hbar} \operatorname{Re} \sum_{\alpha \beta} V_{\alpha \beta} G_{\alpha \beta \eta}^{<}(t, t),
$$

que é uma equação formalmente idêntica à Eq.(3.13), a única diferença está nos índices $\alpha$ e $\beta$. Calculando a equação de movimento para $G_{\alpha \beta \eta}^{<}(t, t)$ vem

$$
G_{\alpha \beta \eta}^{<}(t, t)=\sum_{\alpha^{\prime}} V_{\alpha^{\prime} \beta}^{*} \int d t_{1}\left[G_{\alpha \alpha^{\prime}}^{r}\left(t, t_{1}\right) g_{\beta \eta}^{<}\left(t_{1}, t\right)+G_{\alpha \alpha^{\prime}}^{<}\left(t, t_{1}\right) g_{\beta \eta}^{a}\left(t_{1}, t\right)\right] .
$$

Substituindo (6.10) em (6.9) encontramos

$$
I_{\eta}=\frac{2 e}{\hbar} \operatorname{Re} \sum_{\alpha \alpha^{\prime} \beta} V_{\alpha \beta} V_{\alpha^{\prime} \beta}^{*} \int d t_{1}\left[G_{\alpha \alpha^{\prime}}^{r}\left(t, t_{1}\right) g_{\beta \eta}^{<}\left(t_{1}, t\right)+G_{\alpha \alpha^{\prime}}^{<}\left(t, t_{1}\right) g_{\beta \eta}^{a}\left(t_{1}, t\right)\right] .
$$

Para um Hamiltoniano independente do tempo as funções de Green variam com $t-t_{1}$, portanto a transformada de Fourier de (6.11) leva a

$$
I_{\eta}=\frac{2 e}{\hbar} \operatorname{Re} \sum_{\alpha \alpha^{\prime} \beta} V_{\alpha \beta} V_{\alpha^{\prime} \beta}^{*} \int \frac{d \epsilon}{2 \pi}\left[G_{\alpha \alpha^{\prime}}^{r}(\epsilon) g_{\beta \eta}^{<}(\epsilon)+G_{\alpha \alpha^{\prime}}^{<}(\epsilon) g_{\beta \eta}^{a}(\epsilon)\right] .
$$


Definindo a auto-energia $\Sigma_{\alpha^{\prime} \alpha}^{\eta,<, r, a}=\sum_{\beta} V_{\alpha^{\prime} \beta}^{*} g_{\beta \eta}^{<, r, a}(\epsilon) V_{\alpha \beta}$, onde os super-escritos $<, r, a$ denotam menor, retardada e avançada, respectivamente, a Eq.(6.12) pode ser reescrita da seguinte forma

$$
I_{\eta}=\frac{e}{\hbar} \int \frac{d \epsilon}{2 \pi} \sum_{\alpha \alpha^{\prime}}\left\{\left[G_{\alpha \alpha^{\prime}}^{r}(\epsilon)-G_{\alpha \alpha^{\prime}}^{a}(\epsilon)\right] \Sigma_{\alpha^{\prime} \alpha}^{\eta<}(\epsilon)+G_{\alpha \alpha^{\prime}}^{<}(\epsilon)\left[\Sigma_{\alpha^{\prime} \alpha}^{\eta a}(\epsilon)-\Sigma_{\alpha^{\prime} \alpha}^{\eta r}(\epsilon)\right]\right\} .
$$

Como consideramos o sistema inteiramente separável em $x y$ e $z$, visto que não há interação de Coulomb nem espalhamento de spin, os números quânticos que aparecem em $V_{\alpha \beta}$ devem ser iguais, isto é, $\alpha=\beta \Rightarrow n=m, k_{y}=k_{y \eta}$, e $\sigma=\sigma^{\prime \prime}$. Portanto dizemos que $V_{\alpha \beta}=V_{k_{y} n \sigma, k_{y \eta} k_{z \eta} m \sigma^{\prime \prime}}=\delta_{k_{y} k_{y \eta}} \delta_{n m} \delta_{\sigma \sigma^{\prime \prime}} V$, onde $\delta$ é o delta de Kronecker. Com isso cada componente de spin e cada nível de Landau funciona como um canal de transmissão independente dos demais. Também tomamos $V$ constante, o que simplifica as integrais em $k_{z}$. Com essas simplificações obtemos para a diferença de auto-energias $\Sigma_{\alpha \alpha^{\prime}}^{\eta a}(\epsilon)-\Sigma_{\alpha \alpha^{\prime}}^{\eta r}(\epsilon)$ o seguinte resultado

$$
\begin{aligned}
\Sigma_{\alpha \alpha^{\prime}}^{\eta a}(\epsilon)-\Sigma_{\alpha \alpha^{\prime}}^{\eta r}(\epsilon) & =\sum_{\beta} V_{\alpha \beta}^{*} V_{\alpha^{\prime} \beta}\left[g_{\beta \eta}^{a}(\epsilon)-g_{\beta \eta}^{r}(\epsilon)\right] \\
& =\sum_{\beta} V_{\alpha \beta}^{*} V_{\alpha^{\prime} \beta}\left[\frac{1}{\epsilon-\epsilon_{\beta \eta}-i \delta}-\frac{1}{\epsilon-\epsilon_{\beta \eta}+i \delta}\right] \\
& =i \delta_{k_{y} k_{y}^{\prime}} \delta_{n n^{\prime}} \delta_{\sigma \sigma^{\prime}} \Gamma_{n \sigma}^{\eta}(\epsilon),
\end{aligned}
$$

onde $\Gamma_{n \sigma}^{\eta}=2 \pi|V|^{2} \sum_{k_{z}} \delta\left(\epsilon-\epsilon_{k_{z} n \sigma \eta}\right)$. Para a auto-energia menor $\Sigma^{\eta<}$ temos

$$
\Sigma_{\alpha \alpha^{\prime}}^{\eta<}=\sum_{\beta} V_{\alpha \beta}^{*} V_{\alpha^{\prime} \beta} g_{\beta \eta}^{<}(\epsilon)=i n_{\eta}(\epsilon) \delta_{k_{y} k_{y}^{\prime}} \delta_{n n^{\prime}} \delta_{\sigma \sigma^{\prime}} \Gamma_{n \sigma}^{\eta}(\epsilon) .
$$

Com isso, a corrente pode ser expressa na forma abaixo

$$
I_{\eta}=\frac{i e}{\hbar} g_{B} \int \frac{d \epsilon}{2 \pi} \sum_{n \sigma} \Gamma_{n \sigma}^{\eta}(\epsilon)\left\{n_{\eta}\left[G_{n \sigma}^{r}(\epsilon)-G_{n \sigma}^{a}(\epsilon)\right]+G_{n \sigma}^{<}(\epsilon)\right\},
$$

onde $g_{B}$ é a degenerescência de Landau, $g_{B}=\sum_{k_{y}}=L_{x} L_{y} \frac{e B}{h}$. Note que as funções de Green estão com sub-escritos $n \sigma$ ao invés de $\alpha \alpha^{\prime}$, e temos somas em $n$ e $\sigma$ ao invés de $\alpha \alpha^{\prime}$. Isso deve-se às deltas de Kronecker provenientes de $V_{\alpha \beta}$, que simplificam a soma. Vamos ainda reescrever a Eq.(6.16) como segue

$$
\frac{I_{\eta}}{A J_{0}}=\hbar \omega_{c} \sum_{n \sigma} \int d \epsilon \Gamma_{n \sigma}^{\eta}(\epsilon) i\left\{n_{\eta}\left[G_{n \sigma}^{r}(\epsilon)-G_{n \sigma}^{a}(\epsilon)\right]+G_{n \sigma}^{<}(\epsilon)\right\}
$$

onde $A=L_{x} L_{y}$, e $J_{0}=e m /\left(4 \pi^{2} \hbar^{3}\right)$. Utilizamos as constantes $A$ e $J_{0}$ para preservarmos a notação usual da literatura [38]. Observe ainda que $A J_{0}=e A \nu_{2} / 2 \pi \hbar$, onde $\nu_{2}=m / 2 \pi \hbar^{2}$ é a densidade de estados de um gás de elétrons bidimensional.

\subsection{Shot noise}

Nesta seção discutimos em linhas gerais a dedução da equação do ruído. Conforme fizemos no Cap. 2, calculamos o ruído a partir da definição $S_{\eta \eta^{\prime}}=\left\langle\left\{\widehat{I}_{\eta}(t), \widehat{I}_{\eta^{\prime}}\left(t^{\prime}\right)\right\}\right\rangle-$ 
$2 I_{\eta}^{2}$. Sendo o operador corrente dado por $\widehat{I}_{\eta}=\frac{i e}{\hbar} \sum_{\alpha \beta} V_{\alpha \beta} c_{\beta \eta}^{\dagger} d_{\alpha}-\frac{i e}{\hbar} \sum_{\alpha \beta} V_{\alpha \beta}^{*} d_{\alpha}^{\dagger} c_{\beta \eta}$, podemos reescrever a definição do ruído como segue

$$
\begin{aligned}
S_{\eta \eta^{\prime}}= & \frac{e^{2}}{\hbar} \sum_{\alpha \beta} \sum_{\alpha^{\prime} \beta^{\prime}}\left\{V_{\alpha \beta} V_{\alpha^{\prime} \beta^{\prime}} g_{1}^{(2)>}\left(t, t^{\prime}\right)-V_{\alpha \beta} V_{\alpha^{\prime} \beta^{\prime}}^{*} g_{2}^{(2)>}\left(t, t^{\prime}\right)\right. \\
& \left.\quad-V_{\alpha \beta}^{*} V_{\alpha^{\prime} \beta^{\prime}} g_{3}^{(2)>}\left(t, t^{\prime}\right)+V_{\alpha \beta}^{*} V_{\alpha^{\prime} \beta^{\prime}}^{*} g_{4}^{(2)>}\left(t, t^{\prime}\right)+\text { h.c. }-2 I_{\eta}^{2}\right\},
\end{aligned}
$$

onde as funções de Green $g_{i}^{(2)>}$ são definidas por

$$
\begin{aligned}
& g_{1}^{(2)>}\left(t, t^{\prime}\right)=i^{2}\left\langle c_{\beta \eta}^{\dagger}(t) d_{\alpha}(t) c_{\beta^{\prime} \eta^{\prime}}^{\dagger}\left(t^{\prime}\right) d_{\alpha^{\prime}}\left(t^{\prime}\right)\right\rangle \\
& g_{2}^{(2)>}\left(t, t^{\prime}\right)=i^{2}\left\langle c_{\beta \eta}^{\dagger}(t) d_{\alpha}(t) d_{\alpha^{\prime}}^{\dagger}\left(t^{\prime}\right) c_{\beta^{\prime} \eta^{\prime}}\left(t^{\prime}\right)\right\rangle \\
& g_{3}^{(2)>}\left(t, t^{\prime}\right)=i^{2}\left\langle d_{\alpha}^{\dagger}(t) c_{\beta \eta}(t) c_{\beta^{\prime} \eta^{\prime}}^{\dagger}\left(t^{\prime}\right) d_{\alpha^{\prime}}\left(t^{\prime}\right)\right\rangle \\
& g_{4}^{(2)>}\left(t, t^{\prime}\right)=i^{2}\left\langle d_{\alpha}^{\dagger}(t) c_{\beta \eta}(t) d_{\alpha^{\prime}}^{\dagger}\left(t^{\prime}\right) c_{\beta^{\prime} \eta^{\prime}}\left(t^{\prime}\right)\right\rangle .
\end{aligned}
$$

Observe a semelhança formal entre essas equações e as do Cap. 3. A única diferença está nos índices quânticos, visto que aqui temos $\alpha$ e $\beta$, e no Cap. 3 tínhamos $\sigma$. O cálculo do ruído segue de maneira idêntica à desenvolvida anteriormente, e o resultado obtido está dado abaixo

$$
\begin{aligned}
S_{L L}(0) & =\frac{2 e^{2}}{\hbar} g_{B} \sum_{n \sigma} \int \frac{d \epsilon}{2 \pi}\left[i n_{L}(\epsilon) \Gamma_{n \sigma}^{L}(\epsilon) G_{n \sigma}^{>}(\epsilon)-i\left(1-n_{L}\right) \Gamma_{n \sigma}^{L}(\epsilon) G_{n \sigma}^{<}(\epsilon)\right] \\
& +G_{n \sigma}^{>}(\epsilon) \Gamma_{n \sigma}^{L}(\epsilon) G_{n \sigma}^{<}(\epsilon) \Gamma_{n \sigma}^{L}(\epsilon) \\
& +\left[G_{n \sigma}^{r}(\epsilon)-G_{n \sigma}^{a}(\epsilon)\right] \Gamma_{n \sigma}^{L}(\epsilon)\left[n_{L}(\epsilon) G_{n \sigma}^{>}(\epsilon)-\left(1-n_{L}\right) G_{n \sigma}^{<}(\epsilon)\right] \Gamma_{n \sigma}^{L}(\epsilon) \\
& -\left(1-n_{L}(\epsilon)\right) n_{L}(\epsilon)\left[G_{n \sigma}^{a}(\epsilon) \Gamma_{n \sigma}^{L}(\epsilon) G_{n \sigma}^{a}(\epsilon) \Gamma_{n \sigma}^{L}(\epsilon)+G_{n \sigma}^{r}(\epsilon) \Gamma_{n \sigma}^{L}(\epsilon) G_{n \sigma}^{r}(\epsilon) \Gamma_{n \sigma}^{L}(\epsilon)\right]
\end{aligned}
$$

A Eq.(6.20) assemelha-se ao resultado encontrado para o sistema FM-PQ-FM [Eq.(3.43)]. Aqui porém temos o fator multiplicativo $g_{B}$, as funções $\Gamma_{n \sigma}^{L}(\epsilon)$ que não são constantes, e as funções de Green que são diagonais nos índices de spin e de Landau, visto que desprezamos espalhamentos que acoplem diferentes canais. Ressaltamos ainda que não consideramos interação elétron-elétron neste capítulo. As funções de Green $G_{n \sigma}^{a, r,<,>}(\epsilon)$, assim como $\Gamma_{n \sigma}^{L}(\epsilon)$ serão calculados nas seções subseqüentes. Podemos ainda escrever o ruído em termos de $A J_{0}$, em analogia à corrente [Eq.(6.17)], e com isso vem

$$
\begin{aligned}
\frac{S_{L L}(0)}{2 e J_{0} A} & =\hbar \omega_{c} \sum_{n \sigma} \int d \epsilon\left[i n_{L}(\epsilon) \Gamma_{n \sigma}^{L}(\epsilon) G_{n \sigma}^{>}(\epsilon)-i\left(1-n_{L}\right) \Gamma_{n \sigma}(\epsilon)^{L} G_{n \sigma}^{<}(\epsilon)\right] \\
& +G_{n \sigma}^{>}(\epsilon) \Gamma_{n \sigma}^{L}(\epsilon) G_{n \sigma}^{<}(\epsilon) \Gamma_{n \sigma}^{L}(\epsilon) \\
& +\left[G_{n \sigma}^{r}(\epsilon)-G_{n \sigma}^{a}(\epsilon)\right] \Gamma_{n \sigma}^{L}(\epsilon)\left[n_{L}(\epsilon) G_{n \sigma}^{>}(\epsilon)-\left(1-n_{L}\right) G_{n \sigma}^{<}(\epsilon)\right] \Gamma_{n \sigma}^{L} \\
& -\left(1-n_{L}(\epsilon)\right) n_{L}(\epsilon)\left[G_{n \sigma}^{a}(\epsilon) \Gamma_{n \sigma}^{L}(\epsilon) G_{n \sigma}^{a}(\epsilon) \Gamma_{n \sigma}^{L}(\epsilon)+G_{n \sigma}^{r}(\epsilon) \Gamma_{n \sigma}^{L}(\epsilon) G_{n \sigma}^{r}(\epsilon) \Gamma_{n \sigma}^{L}(\epsilon)\right] .
\end{aligned}
$$

Observe que a equação acima assemelha-se à Eq.(3.43). Aqui, porém, temos uma equação escalar, enquanto a Eq.(3.43) é matricial, e o campo magnético introduz 
um fator adicional (degenerescência de Landau) ${ }^{5}$. A partir das Eqs.(6.17) e (6.21) derivamos as equações de Landauer-Büttiker para corrente e ruído, conforme discutiremos posteriormente. Para isso porém, precisamos conhecer as funções de Green do poço quântico.

\subsection{Funções de Green do poço quântico}

As funções de Green $G_{\alpha \alpha^{\prime}}^{r}, G_{\alpha \alpha^{\prime}}^{a}, G_{\alpha \alpha^{\prime}}^{<}$e $G_{\alpha \alpha^{\prime}}^{>}$são obtidas através da continuação analítica da função de Green ordenada no contorno $G_{\alpha \alpha^{\prime}}\left(\tau, \tau^{\prime}\right)=-i\left\langle T_{c} d_{\alpha}(\tau) d_{\alpha^{\prime}}^{\dagger}\left(\tau^{\prime}\right)\right\rangle$, que será calculada via o método de equação de movimento. Como há uma semelhança formal entre $G_{\alpha \alpha^{\prime}}\left(\tau, \tau^{\prime}\right)$ e a função de Green de tempo real $G_{\alpha \alpha^{\prime}}^{t}\left(t, t^{\prime}\right)=$ $-i\left\langle T d_{\alpha}(t) d_{\alpha^{\prime}}^{\dagger}\left(t^{\prime}\right)\right\rangle$, calculamos primeiro a equação de movimento para $G_{\alpha \alpha^{\prime}}^{t}\left(t, t^{\prime}\right)$ e depois escrevemos uma equação idêntica para a função de Green ordenada no contorno. Calculando a derivada temporal de $G_{\alpha \alpha^{\prime}}^{t}\left(t, t^{\prime}\right)$ vem

$$
\left(i \frac{\partial}{\partial t}-\epsilon_{\alpha}\right) G_{\alpha \alpha^{\prime}}^{t}\left(t, t^{\prime}\right)=\delta_{\alpha \alpha^{\prime}} \delta\left(t-t^{\prime}\right)+\int d t_{1} \Sigma_{\alpha \alpha_{1}}^{t}\left(t, t_{1}\right) G_{\alpha_{1} \alpha^{\prime}}^{t}\left(t_{1}, t^{\prime}\right)
$$

onde a auto-energia é definida como $\Sigma_{\alpha \alpha_{1}}^{t}\left(t, t_{1}\right)=\sum_{\beta \eta} V_{\alpha_{1} \beta}^{*} g_{\beta \sigma \eta}\left(t, t_{1}\right) V_{\alpha \beta}$. Definindo a função de Green do poço quântico não acoplado a contatos $\left(i \frac{\partial}{\partial t}-\epsilon_{\alpha}\right) G_{\alpha \alpha^{\prime}}^{t 0}\left(t, t_{1}\right)=$ $\delta_{\alpha \alpha^{\prime}} \delta\left(t-t^{\prime}\right)$, podemos reescrever a Eq.(6.22) em sua forma integral (equação de Dyson)

$$
G_{\alpha \alpha^{\prime}}^{t}\left(t, t^{\prime}\right)=G_{\alpha \alpha^{\prime}}^{0 t}\left(t, t^{\prime}\right)+\sum_{\alpha_{1} \alpha_{2}} \iint d t_{1} d t_{2} G_{\alpha \alpha_{1}}^{0 t}\left(t, t_{1}\right) \Sigma_{\alpha_{1} \alpha_{2}}^{t}\left(t_{1}, t_{2}\right) G_{\alpha_{2} \alpha^{\prime}}^{t}\left(t_{2}, t^{\prime}\right) .
$$

Agora reescrevemos a Eq.(6.23) em termos de variáveis complexas $\tau$, $\tau^{\prime}$, de modo a se obter a função de Green ordenada no contorno. Com isso vem

$$
G_{\alpha \alpha^{\prime}}\left(\tau, \tau^{\prime}\right)=G_{\alpha \alpha^{\prime}}^{0}\left(\tau, \tau^{\prime}\right)+\sum_{\alpha_{1} \alpha_{2}} \iint d \tau_{1} d \tau_{2} G_{\alpha \alpha_{1}}^{0}\left(\tau, \tau_{1}\right) \Sigma_{\alpha_{1} \alpha_{2}}\left(\tau_{1}, \tau_{2}\right) G_{\alpha_{2} \alpha^{\prime}}\left(\tau_{2}, \tau^{\prime}\right)
$$

Efetuando agora a continuação analítica e a transformada de Fourier, obtemos equações de Dyson para as funções de Green retardada e avançada,

$$
\begin{aligned}
& G_{n \sigma}^{r}(\epsilon)=G_{n \sigma}^{0 r}(\epsilon)+G_{n \sigma}^{0 r}(\epsilon) \Sigma_{n \sigma}^{r}(\epsilon) G_{n \sigma}^{r}(\epsilon) \rightarrow G_{n \sigma}^{r}(\epsilon)=\frac{1}{G_{n \sigma}^{0 r^{-1}}-\Sigma^{r}} \\
& G_{n \sigma}^{a}(\epsilon)=G_{n \sigma}^{0 a}(\epsilon)+G_{n \sigma}^{0 a}(\epsilon) \Sigma_{n \sigma}^{a}(\epsilon) G_{n \sigma}^{a}(\epsilon) \rightarrow G_{n \sigma}^{a}(\epsilon)=\frac{1}{G_{n \sigma}^{0 a^{-1}}-\Sigma^{a}}
\end{aligned}
$$

onde $G_{n \sigma}^{0 r^{-1}}(\epsilon)=\epsilon-\epsilon_{n}+i \delta$ e $G_{n \sigma}^{0 a^{-1}}=\epsilon-\epsilon_{n \sigma}-i \delta$, e a equação de Keldysh para a função de Green menor,

$$
G_{n \sigma}^{<}(\epsilon)=G_{n \sigma}^{r}(\epsilon) \Sigma_{n \sigma}^{<}(\epsilon) G_{n \sigma}^{a}(\epsilon)
$$

\footnotetext{
${ }^{5} \mathrm{~A}$ equação (6.21) é a primeira na literatura que usa funções de Green de não equilíbrio para transporte em sistemas com terminais DMS na presença de campo magnético.
} 
Para as auto-energias temos as seguintes expressões $\Sigma^{r}(\epsilon)=-\frac{i}{2} \Gamma_{n \sigma}=-\frac{i}{2}\left[\Gamma_{n \sigma}^{L}(\epsilon)+\right.$ $\left.\Gamma_{n \sigma}^{R}(\epsilon)\right]$, e $\Sigma_{n \sigma}^{<}(\epsilon)=i\left[n_{L}(\epsilon) \Gamma_{n \sigma}^{L}(\epsilon)+n_{R}(\epsilon) \Gamma_{n \sigma}^{R}(\epsilon)\right]$. Com isso podemos escrever

$$
G_{n \sigma}^{<}(\epsilon)=i A_{n \sigma}(\epsilon) \bar{f}_{n \sigma}(\epsilon)
$$

onde

$$
\bar{f}_{n \sigma}(\epsilon)=\frac{n_{L}(\epsilon) \Gamma_{n \sigma}^{L}(\epsilon)+n_{R}(\epsilon) \Gamma_{n \sigma}^{R}(\epsilon)}{\Gamma_{n \sigma}^{L}(\epsilon)+\Gamma_{n \sigma}^{R}(\epsilon)},
$$

e

$$
A_{n \sigma}(\epsilon)=\frac{1}{\left(\epsilon-\epsilon_{n \sigma}\right)^{2}+\left(\frac{\Gamma_{n \sigma}^{L}+\Gamma_{n \sigma}^{R}}{2}\right)^{2}} .
$$

Vemos então que $G_{n \sigma}^{<}(\epsilon)$ apresenta uma forma de pseudo-equilibrium (ou quase equilíbrio) com uma "função de Fermi" $\bar{f}_{n \sigma}(\epsilon)^{6}$.

Seguimos aplicando essas equações para fazer uma conexão entre o formalismo de funções de Green de não-equilíbrio com a formulação de Landauer-Büttiker.

Com as equações desta seção a descrição matemática do transporte está quase completa. Só nos falta calcular a função densidade de estados $\Gamma_{n \sigma}^{\eta}(\epsilon)$, o que está feito na próxima seção.

\subsection{Conexão com Landauer-Büttiker}

Aplicando, nas Eqs.(6.17) e (6.21), as funções de Green deduzidas na seção anterior, podemos obter as equações de Landauer-Büttiker para corrente e ruído. Por simplicidade de cálculo, vamos assumir temperaturas suficientemente baixas de modo que $n_{L}\left(1-n_{L}\right) \approx 0$ e $n_{R}\left(1-n_{L}\right) \approx 0$. Com isso mostramos que

$$
\frac{I_{L}}{A J_{0}}=\hbar \omega_{c} \int d \epsilon \sum_{n \sigma} T_{n \sigma}(\epsilon)\left(n_{L}-n_{R}\right)
$$

e a Eq.(6.21) resulta em

$$
\frac{S}{2 e A J_{0}}=\hbar \omega_{c} \int d \epsilon \sum_{n \sigma} T_{n \sigma}(\epsilon)\left[1-T_{n \sigma}(\epsilon)\right]\left(n_{L}-n_{R}\right)^{2},
$$

onde o coeficiente de transmissão é definido da seguinte forma

$$
T_{n \sigma}(\epsilon)=\frac{\Gamma_{n \sigma}^{L} \Gamma_{n \sigma}^{R}}{\left(\epsilon-\epsilon_{n}\right)^{2}+\left(\frac{\Gamma_{n \sigma}^{L}+\Gamma_{n \sigma}^{R}}{2}\right)^{2}} .
$$

Este coeficiente de transmissão nada mais é do que a fórmula de Breit-Wigner [64]. As expressões (6.31) e (6.32) constituem exatamente as equações de LandauerBüttiker para a corrente e shot noise [64]. Seguimos calculando uma equação para $\Gamma_{n \sigma}^{\eta}(\epsilon)$.

\footnotetext{
${ }^{6}$ Uma distribuição de pseudo-equilíbrio como essa também foi obtida por A. P. Jauho [57] em um sistema de ponto quântico com um nível ressonante e não interagente.
} 


\subsection{Funções $\Gamma_{n \sigma}^{L}(\epsilon)$ e $\Gamma_{n \sigma}^{R}(\epsilon)$}

Calculamos agora a função $\Gamma_{n \sigma}^{\eta}(\epsilon)$, que é, a menos da constante multiplicativa $2 \pi|V|^{2}$, a densidade de estados dos terminais. A partir da definição $\Gamma_{n \sigma}^{\eta}(\epsilon)=$ $2 \pi|V|^{2} \sum_{k_{z}} \delta\left[\epsilon-\epsilon_{\eta}\left(k_{z} n \sigma\right)\right]$ escrevemos

$$
\Gamma_{n \sigma}^{\eta}=2 \pi|V|^{2} \sum_{k_{z}} \delta\left[\epsilon-\frac{\hbar^{2} k_{z}^{2}}{2 m^{*}}-\hbar \omega_{c}\left(n+\frac{1}{2}\right)-E_{s d}-\xi_{\eta} e V\right] .
$$

Passando da soma em $k_{z}$ para uma integral vem

$$
\begin{aligned}
\Gamma_{n \sigma}^{\eta}(\epsilon) & =2 \pi|V|^{2} \frac{L_{z}}{2 \pi} \int_{-\infty}^{\infty} d k_{z} \delta\left[\epsilon-\frac{\hbar^{2} k_{z}^{2}}{2 m^{*}}-\hbar \omega_{c}\left(n+\frac{1}{2}\right)-E_{s d}-\xi_{\eta} e V\right] \\
& =2 \pi|V|^{2} \frac{L_{z}}{2 \pi} \sqrt{\frac{m^{*}}{2 \hbar^{2}}} 2 \int \frac{d \epsilon_{z}}{\sqrt{\epsilon_{z}}} \delta\left[\epsilon-\epsilon_{z}-\hbar \omega_{c}\left(n+\frac{1}{2}\right)-E_{s d}-\xi_{\eta} e V\right] \\
& =\frac{\Upsilon}{\sqrt{\epsilon-\hbar \omega_{c}\left(n+\frac{1}{2}\right)-E_{s d}-\xi_{\eta} e V}}
\end{aligned}
$$

onde $\Upsilon=2|V|^{2} L_{z} \sqrt{\frac{m^{*}}{2 \hbar^{2}}}$. Observe que essa densidade de estados introduz naturalmente um cutoff de energia para $\epsilon<\hbar \omega_{c}\left(n+\frac{1}{2}\right)+E_{s d}+\xi_{\eta} e V$. Note que cada sub-banda de Landau possui um cutoff diferente. No caso $n=0$ temos a menor energia possível igual a $\hbar \omega_{c} / 2+E_{s d}$ para $\eta=L$ e $\hbar \omega_{c} / 2+E_{s d}-e V$ para $\eta=R$. Portanto o mínimo de energia do lado direito $(\mathrm{R})$ está deslocado de $-e V$ com relação ao lado esquerdo (L), em virtude da tensão externa aplicada. Seguimos apresentando os resultados.

\subsection{Parâmetros e Metodologia}

Dentre os parâmetros de nossa análise temos os potenciais químicos dos reservatórios $\mu_{L}$ e $\mu_{R}$, cujos valores adotados são $\mu_{L}=30 \mathrm{meV}$ e $\mu_{R}=\mu_{L}-\mathrm{eV}$. Para a temperatura utilizamos $T=1 \mathrm{~K}$. Para o material DMS temos $x=0.06$, $N_{0} \alpha=0.26 \mathrm{eV}, S=5 / 2$ (valores típicos para o ZnMnSe [90]). Para o acoplamento poço-quântico-terminais adotamos $\Upsilon=1(\mathrm{meV})^{3 / 2}$, e para o nível $\epsilon_{0}$ temos $\epsilon_{0}=30 \mathrm{meV}$. Note que, como $\epsilon_{0}=\mu_{L}$ os níveis de Landau $\hbar \omega_{c}\left(n+\frac{1}{2}\right)$ do poço encontram-se todos acima de $\mu_{L}$ e $\mu_{R}$ para $e V=0$. A condução pelo canal $n=0$ começa somente quando $\mathrm{eV}=\hbar \omega_{c}$.

O algoritmo empregado no cálculo tem os seguintes passos: definimos os parâmetros (conforme listado acima), calculamos as funções de Green $G^{r}$ e $G^{a}$ através das equações de Dyson [Eqs.(6.25)-(6.26)]. Calculamos $G^{<}$via equação de Keldysh [Eq.(6.27)], e $G^{>}$através da identidade $G^{>}=G^{r}-G^{a}+G^{<}$. A função densidade de estados é obtida a partir da Eq.(6.35). Substituimos todas essas equações nas expressões para a corrente [Eq.(6.17)] e ruído [Eq.(6.21)], e então operamos uma integração numérica na energia $\epsilon$. Os resultados obtidos estão ilustrados na seção seguinte. 


\subsection{Resultados}

\subsubsection{Densidade de estados}

Na figura (6.2) ilustramos as somas $\sum_{n} \Gamma_{n \sigma}^{L}(\epsilon)$ e $\sum_{n} \Gamma_{n \sigma}^{R}(\epsilon)$, paineis da esquerda e da direita, respectivamente, e a função espectral do poço quântico $A_{\sigma}(\epsilon)=$ $i \sum_{n}\left[G_{n \sigma}^{r}(\epsilon)-G_{n \sigma}^{a}(\epsilon)\right]$, painéis centrais. Como $\Gamma_{n \sigma}^{L}(\epsilon)$ e $\Gamma_{n \sigma}^{R}(\epsilon)$ são proporcionais as densidades de estado dos seus respectivos reservatórios [Eq.(6.35)], o que vemos na Fig.(6.2) [painéis (a),(c),(d),(f)] são as densidade de estados (DE) a menos de uma constante.

Na figura (6.2) as duas componentes de spin foram resolvidas e estão indicadas pelas setas $\uparrow$ (curvas pretas) e $\downarrow$ (curvas vermelhas). A soma nos níveis de Landau dá origem aos vários picos observados em $A_{\sigma}(\epsilon)$ e nas densidades de estados dos reservatórios, sendo cada pico correspondente a um nível de Landau. Consideramos as tensões $\mathrm{eV}=30 \mathrm{meV}$ (paineis superiores) e $\mathrm{eV}=60 \mathrm{meV}$ (painéis inferiores) pois fornecem diferentes regimes de transporte. Antecipamos por exemplo que, para $\mathrm{eV}=30 \mathrm{meV}$, temos uma corrente $\uparrow$-polarizada, enquanto para $\mathrm{eV}=60 \mathrm{meV}$ obtemos uma corrente $100 \% \downarrow$-polarizada, e portanto temos regimes de polarização distintos.

As curvas indicadas por $n_{L}$ e $n_{R}$ (curvas em verde) são as funções de Fermi do emissor e do coletor, respectivamente. O degrau na função de Fermi indica a posição dos potenciais químicos $\mu_{L}$ e $\mu_{R}$. O potencial químico $\mu_{L}$ está fixo, isto é, não depende da tensão $V$, enquanto o potencial químico $\mu_{R}$ obedece a relação $\mu_{R}=\mu_{L}-e V$ [veja painéis (c) e (f)].

Observe que há um deslocamento rígido da densidade de estados do terminal da direita com relação a densidade de estados do terminal da esquerda. Isso devese a tensão externa aplicada que produz uma renormalização de energia igual a $-e V$ entre os estados $\Gamma_{n \sigma}^{L}(\epsilon)$ e $\Gamma_{n \sigma}^{R}(\epsilon)$, assim como ocorre com o potencial químico $\mu_{R}$. Já para os estados do poço quântico temos uma renormalização de $-e V / 2$, isto é, assumimos que a queda de tensão entre o emissor e o poço é de $e V / 2$. Essa hipótese despreza os efeitos de acumulação de carga no poço quântico. Note que, na Fig.(6.2), fixamos a densidade de estados do emissor (lado esquerdo), e portanto adotamos o emissor como referencial de energia.

Além do deslocamento entre os estados $\Gamma_{n \sigma}^{L}$ e $\Gamma_{n \sigma}^{R}$, gerado pela tensão externa, temos um desdobramento entre as duas componentes de spin $\Gamma_{n \uparrow}^{\eta}$ e $\Gamma_{n \downarrow}^{\eta}$. Esse desdobramento deve-se ao efeito Zeeman gigante, originado na interação de troca $s-d$ quando $B \neq 0$. Nessa figura em particular adotamos $B=10 \mathrm{~T}$. Como para $B=10 \mathrm{~T}$ já estamos na região de saturação da função de Brillouin, podemos estimar o valor do desdobramento das bandas simplesmente tomando a diferença $E_{s d}^{\sigma_{z}=1 / 2}-E_{s d}^{\sigma_{z}=-1 / 2}=x N_{0} \alpha \frac{5}{2}=18.6 \mathrm{meV}$, o que está de acordo com o espaçamento observado na Fig.(6.2). Como no poço não há material DMS, o efeito Zeeman 
gigante só aparece nos terminais.
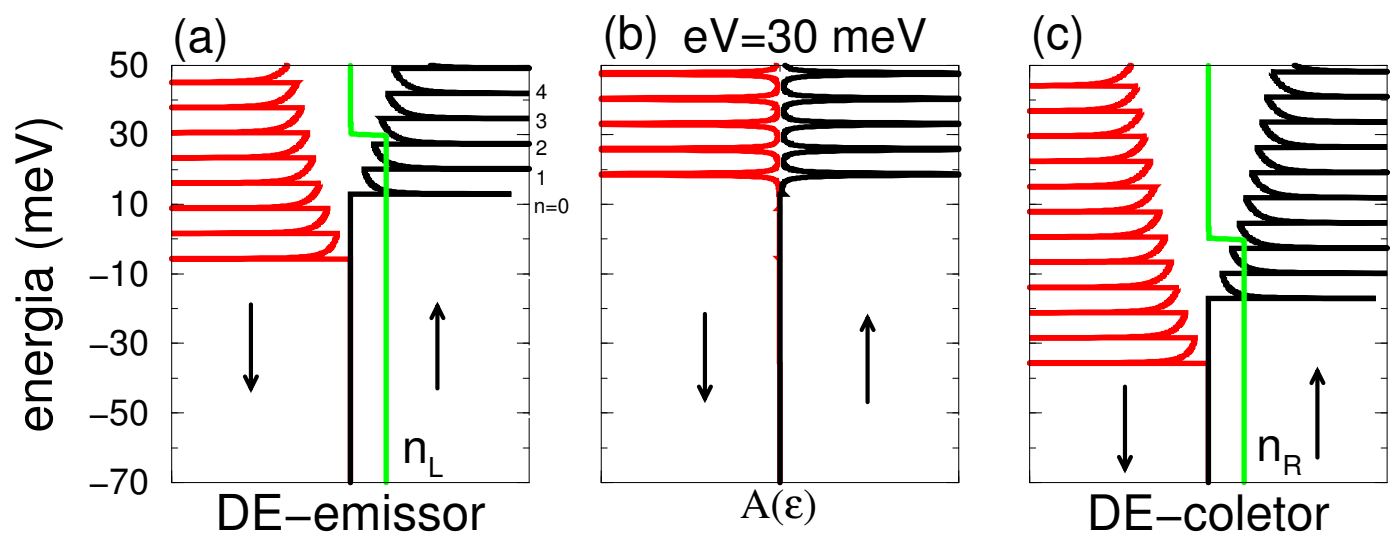

DE-coletor
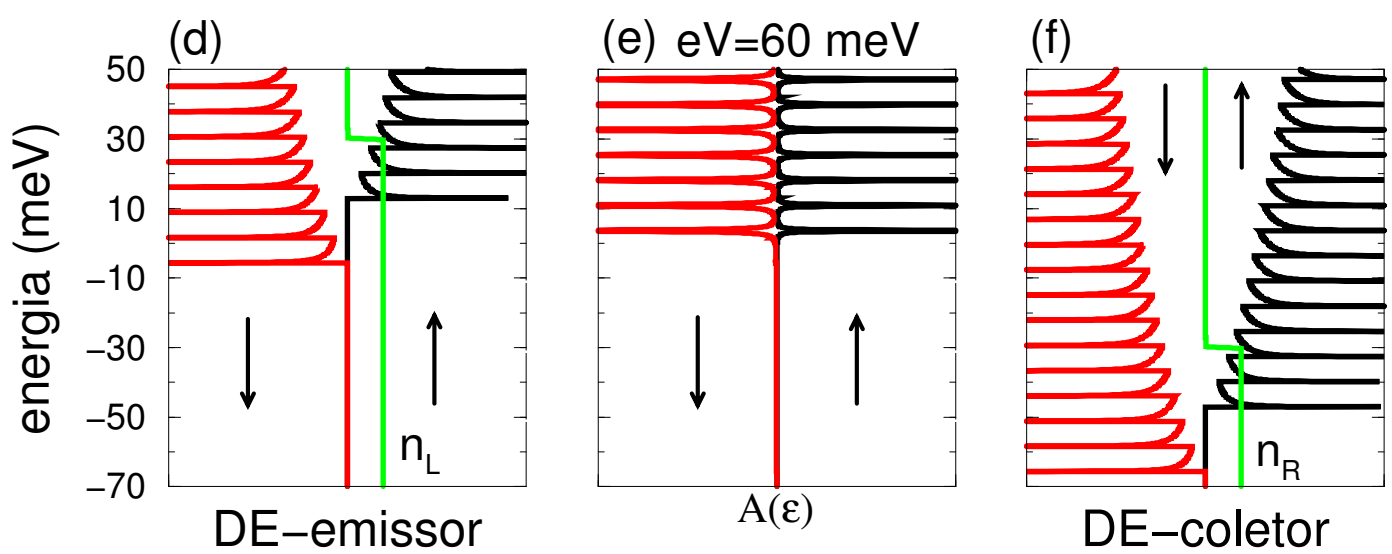

Figura 6.2: Densidade de estados (DE) do emissor (a) e (d), do poço quântico (b) e (e) e do coletor (c) e (f) para um campo magnético fixo $(B=10 \mathrm{~T})$ e duas tensões $(\mathrm{eV}=30 \mathrm{meV}$ e $\mathrm{eV}=60 \mathrm{meV})$. Graficamos separadamente as duas componentes de spin (curvas pretas para spin $\uparrow$ e vermelhas para spin $\downarrow$ ). As linhas verdes são as funções de Fermi $n_{L}$ e $n_{R}$. As densidades de estado dos reservatórios são somas do tipo $\sum_{n} 1 / \sqrt{\epsilon-\left(\epsilon_{c o}+n \hbar \omega_{c}\right)}$, onde $\epsilon_{c o}$ é uma energia de corte que depende do spin e da tensão . No lado emissor temos que, para $n=0$, há uma energia de corte em torno de $12 \mathrm{meV}$ para spin $\uparrow$ e $-6 \mathrm{meV}$ para spin $\downarrow$. Para o lado coletor temos essas energias de corte deslocadas de $-e V$. Para o poço a DE são lorentzianas espaçadas de $\hbar \omega_{c}$ e larguras $\Gamma_{n \sigma}^{L}+\Gamma_{n \sigma}^{R}$. O deslocamento vertical entre as bandas para spin $\uparrow$ e $\downarrow$ dos reservatórios deve-se a interação de troca $s$ - $d$, que aumenta a energia para spins $\uparrow$ e diminui para spins $\downarrow$. A diferença entre $\mu_{L}$ e $\mu_{R}$ é dada por $\mu_{L}-\mu_{R}=e V$.

Vale salientar que a forma da densidade de estados dos reservatórios é resultado da soma em $n$ da função $\Upsilon / \sqrt{\epsilon-\hbar \omega_{c}\left(n+\frac{1}{2}\right)-E_{s d}-\xi_{\eta} e V}$. Portanto o que vemos na Fig. (6.2) é uma soma do tipo $\frac{1}{\sqrt{\epsilon-\epsilon_{c o}}}+\frac{1}{\sqrt{\epsilon-\left(\epsilon_{c o}+\hbar \omega_{c}\right)}}+\frac{1}{\sqrt{\epsilon-\left(\epsilon_{c o}+2 \hbar \omega_{c}\right)}}+\ldots$, onde $\epsilon_{c o}$ dá o corte de energia (cutoff) em $\Gamma_{n \sigma}^{\eta}$. Note na Fig.(6.2) que $\Gamma_{n \sigma}^{\eta}(\epsilon)=0$ para valores de $\epsilon$ abaixo da respectiva energia $\hbar \omega_{c}(n+0.5)+E_{s d}+\xi_{\eta} e V$ (posição onde ocorre um pico). Isso está claramente observado para o menor nível de Landau 
$(n=0)$, onde vemos que abaixo do pico correspondente a $n=0$ temos $\Gamma_{0 \sigma}^{L}(\epsilon)=0$, ou seja não há estados disponíveis (lembre-se que $\Gamma_{n \sigma}^{\eta}(\epsilon)$ é proporcional à densidade de estados). Ao contrário do reservatório, no poço obtemos funções lorentzianas espaçadas de $\hbar \omega_{c}$, sendo a largura de cada lorentziana dada por $\Gamma_{n \sigma}^{L}+\Gamma_{n \sigma}^{R}$.

Na figura (6.3) ilustramos apenas os estados correspondentes a $n=0$ no emissor e no poço para $e V=30 \mathrm{meV}$ e $e V=60 \mathrm{meV}$. Para $e V=30 \mathrm{meV}$ os estados $A_{n=0 \uparrow}(\epsilon)$ estão numa faixa de energia em que $\Gamma_{0 \sigma}^{L}(\epsilon) \neq 0$, e o mesmo se dá para $A_{n=0 \downarrow}(\epsilon)$. Note que os estados $A_{n=0 \uparrow}$ possuem um maior overlap com os estados $\uparrow$ do emissor do que os estados $\downarrow$-compare as regiões achuradas para spin $\uparrow$ e $\downarrow$. Como em nosso modelo os elétrons fluem através da junção conservando os números quânticos $n$ e $\sigma$, isto é, assumimos que não há espalhamento de spin e de momento, o tunelamento eletrônico de um elétron do emissor para o poço só é possível quando há algum overlap entre o $n$-ésimo nível do poço com o $n$-ésimo estado do emissor. Portanto os overlaps ilustrados na Fig.(6.3) são essenciais para que haja transporte. Em particular, para $\mathrm{eV}=30 \mathrm{meV}$ a corrente e o ruído para spins $\uparrow$ são maiores do que para spin $\downarrow$, em virtude do overlap para spins $\uparrow$ ser maior do que para spins $\downarrow$.

Já para $e V=60 \mathrm{meV}$ [painel (b)] vemos que o nível $n=0$ para spin $\uparrow$ está numa faixa de energia tal que $\Gamma_{0 \uparrow}^{L}(\epsilon)=0$ [região do cutoff]. Portanto não há ressonância entre os estados $\uparrow$ do poço com os estados $\uparrow$ do emissor, e conseqüentemente não pode haver corrente $\uparrow$. Em contrapartida, o nível $n=0$ para spin $\downarrow$ está numa faixa de energia em que $\Gamma_{0 \downarrow}^{L}(\epsilon) \neq 0$, havendo assim ressonância para spin $\downarrow$, e conseqüentemente corrente. Note que, de $\mathrm{eV}=30 \mathrm{meV}$ para $\mathrm{eV}=60 \mathrm{meV}$ o pico $A_{n=0 \uparrow}$ sofre um estreitamento, significando que, com o aumento da tensão, o acoplamento poço-quântico-emissor atenuou-se para spins $\uparrow$. O pico $A_{n=0 \uparrow}$ não adquire uma largura zero visto que, muito embora não haja acoplamento com o emissor para $\mathrm{eV}=60 \mathrm{meV}$, o acoplamento com o reservatório coletor permanece, mantendo assim um alargamento finito do pico. Já o nível $A_{n=0 \downarrow}$ alarga-se com o aumento da tensão indicando que o acoplamento poço-quântico-emissor intensificase para a componente $\downarrow$ do spin. Eventualmente para tensão grande o suficiente $(e V \approx 80 \mathrm{meV})$, os estados $A_{n=0 \downarrow}$ entram na faixa de energia do cutoff $\left(\Gamma_{n=0 \downarrow}^{L}=0\right)$, resultando num anulamento da corrente $I_{\downarrow}$. Veremos com maior detalhe esses efeitos nos gráficos para a corrente e ruído ilustrados a seguir. 


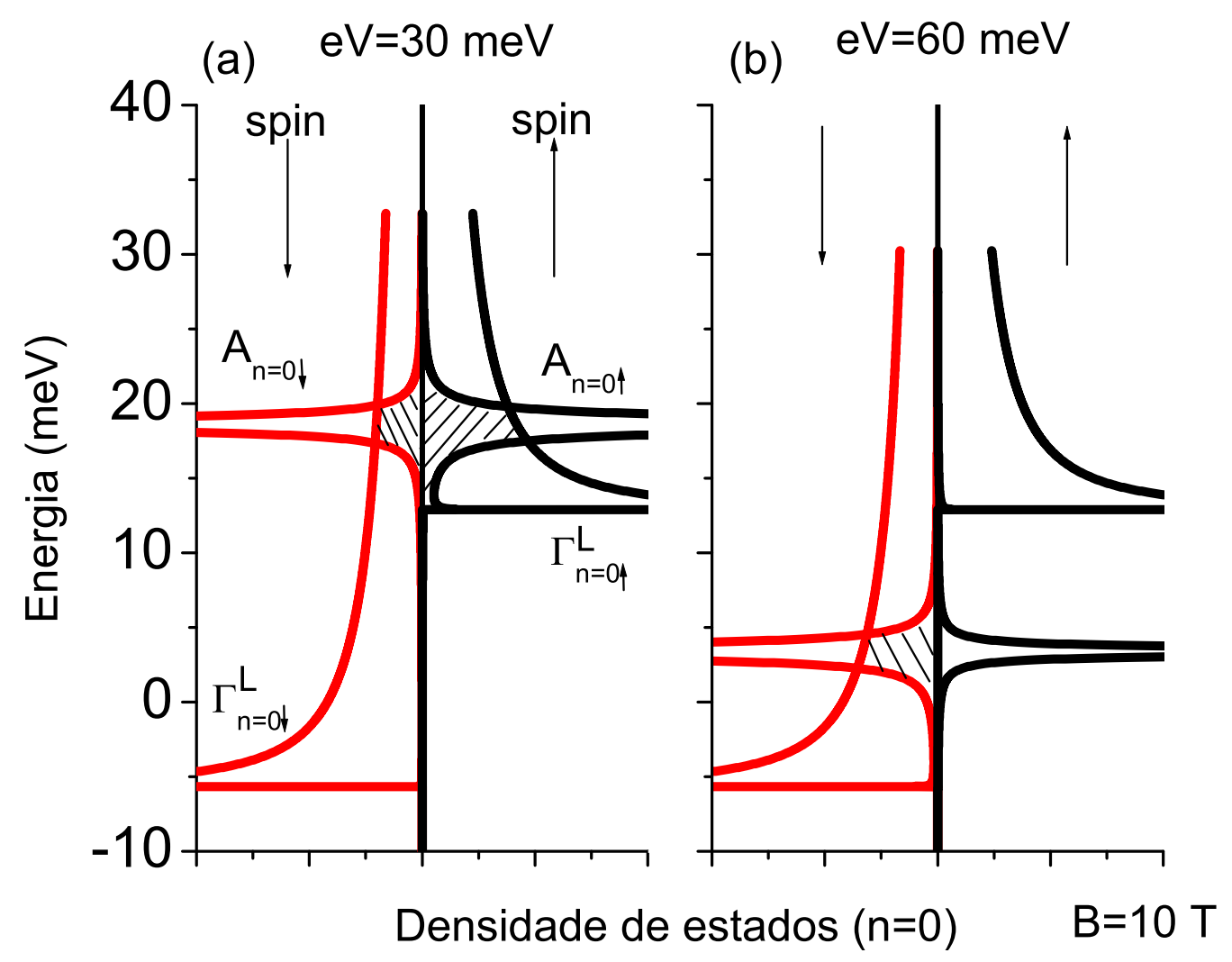

Figura 6.3: Densidade de estados para $n=0$. Enquanto na figura (6.2) apresentamos uma soma em $n$, aqui ilustramos apenas $n=0$, para o lado emissor e o poço quântico. Observe que para $\mathrm{eV}=30 \mathrm{meV}$ [painel (a)] temos que os picos de $A_{n=0 \uparrow}$ e $A_{n=0 \downarrow}$ apresentam overlap com os estados do emissor. Como esse overlap é maior para spins $\uparrow$ (compare regiões achuradas para spins $\uparrow$ e $\downarrow$ ), temos que a largura do pico $A_{n=0 \uparrow}$ é maior do que do pico $A_{n=0 \downarrow}$. Já para $e V=60 \mathrm{meV}$ [painel (b)] temos que o overlap entre $A_{n=0 \uparrow}$ e os estados $\uparrow$ do emissor é nulo, o que dá origem a um estreitamento do pico. Em contraste, o estado $A_{n=0 \downarrow}$ aumenta o seu overlap com os estados do emissor quando passamos de $\mathrm{eV}=30 \mathrm{meV}$ para $\mathrm{eV}=60 \mathrm{meV}$, resultando num pequeno alargamento do pico para spin $\downarrow$. 


\subsubsection{Corrente e ruído como função de $V$}

Na figura (6.4) mostramos gráficos para a corrente, o ruído e o fator de Fano como função da tensão externa e campo magnético fixo $(B=10 \mathrm{~T})$. A corrente e o ruído estão resolvidos em spin, isto é, graficamos separadamente $I_{\uparrow}, I_{\downarrow}, S_{\uparrow}$ e $S_{\downarrow}$. Contrastamos os casos com e sem material DMS nos eletrodos, de modo a observar a influência do efeito Zeeman gigante sobre o transporte. As curvas pretas correspondem ao caso sem material DMS, enquanto as curvas vermelhas ao caso com material DMS nos terminais.

Na ausência de material DMS, isto é, caso não-magnético (NMS), as correntes $I_{\uparrow}$ e $I_{\downarrow}$ e os ruídos $S_{\uparrow}$ e $S_{\downarrow}$ são iguais entre si, isto é, $I_{\uparrow}=I_{\downarrow}$ e $S_{\uparrow}=S_{\downarrow}$. Ambos apresentam três plateaus inteiros e um pequeno plateau imediatamente antes da corrente e do ruído caírem a zero. Essas grandezas vão a zero para $\mathrm{eV} \gtrsim 60 \mathrm{meV}$ em virtude dos níveis do poço entrarem na região de energia em que $\Gamma_{n \sigma}^{L}(\epsilon)=0$ para $\mathrm{eV} \gtrsim 60 \mathrm{meV}$. Dizemos então que os níveis do poço saíram de ressonância com os estados do emissor [95].

Na presença de material DMS e campo magnético não nulo, o desdobramento das bandas de energia para spins $\uparrow$ e $\downarrow$ leva a uma polarização da corrente e do ruído, isto é, $I_{\uparrow} \neq I_{\downarrow}$ e $S_{\uparrow} \neq S_{\downarrow}$. Para $e V \lesssim 40 \mathrm{meV}$ vemos que $I_{\uparrow}>I_{\downarrow}$, portanto a corrente é $\uparrow$-polarizada. Quando $e V \gtrsim 40 \mathrm{meV}$ a corrente $I_{\uparrow}$ e o ruído $S_{\uparrow}$ vão a zero, visto que o sistema sai de ressonância como ilustrado na Fig.6.3(b). Com isso a corrente torna-se $\downarrow$-polarizada para tensões $\mathrm{eV} \gtrsim 40 \mathrm{meV}$. Como o fundo da banda de condução para spin $\downarrow$ está alguns meV mais baixo, em virtude do efeito Zeeman gigante, a corrente para spins $\downarrow$ perdura até tensões maiores. Quando $e V \approx 80 \mathrm{meV}$ a corrente e o ruído $\downarrow$ também vão a zero, visto que os níveis do poço entram na faixa de energia tal que $\Gamma_{n \downarrow}^{L}(\epsilon)=0$. 

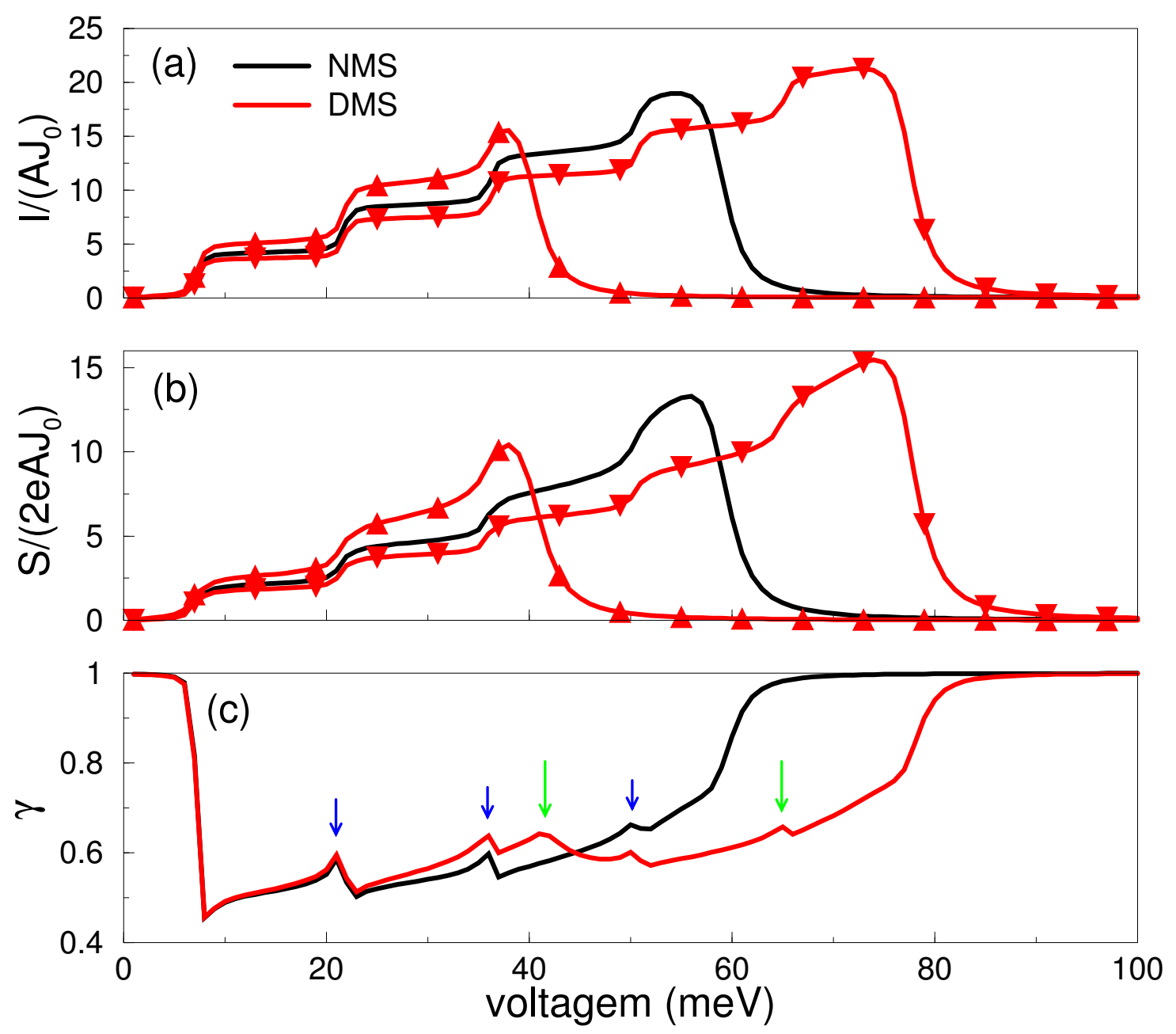

Figura 6.4: (a) Correntes $I_{\uparrow}(\Delta)$ e $I_{\downarrow}(\nabla)$, (b) ruídos $S_{\uparrow}(\triangle)$ e $S_{\downarrow}(\nabla)$, e (c) fator de Fano como função da tensão externa $\mathrm{eV}$. As curvas vermelhas com triangulos $\Delta \mathrm{e} \nabla$ correspondem ao caso DMS. As curvas pretas indicam o caso não magnéticos (NMS). Com contatos DMS há um desdobramento de bandas que resulta numa polarização da corrente $\left(I_{\uparrow} \neq I_{\downarrow}\right)$ e do ruído $\left(S_{\uparrow} \neq S_{\downarrow}\right)$. Observe que devido a esse desdobramento há uma faixa de tensão $(\mathrm{eV}>40 \mathrm{meV})$ para a qual $I_{\uparrow}=0$ e $S_{\uparrow}=0$, enquanto $I_{\downarrow} \neq 0$ e $S_{\downarrow} \neq 0$, resultando numa máxima polarização do transporte. Cada degrau na corrente e no ruído está associado a um nível de Landau. Em particular temos $n=0,1,2$ para spin $\uparrow$ e $n=0,1,2,3,4$ para spin $\downarrow$. O fator de Fano $\gamma=\left(S_{\uparrow}+S_{\downarrow}\right) / 2 e\left(I_{\uparrow}+I_{\downarrow}\right)$ [painel (c)] apresenta picos sempre que um novo nível de Landau entra em ressonância com o emissor (isto é, fica abaixo de $\mu_{L}$ ). No caso de contatos não magnéticos observamos três picos correspondentes aos níveis $n=1,2,3$ (setas azuis). Com contatos DMS surgem dois picos adicionais (setas verdes), sendo um correspondente ao canal $n=4$ para spin $\downarrow$ e o outro correspondente ao fechamento dos canais para spin $\uparrow$ (pico em $40 \mathrm{meV}$ ). Portanto o fator de Fano apresenta picos não só quando um novo nível de Landau entra em ressonância, mas também quando esses níveis saem de ressonância. O campo utilizado aqui foi de 10 T. 


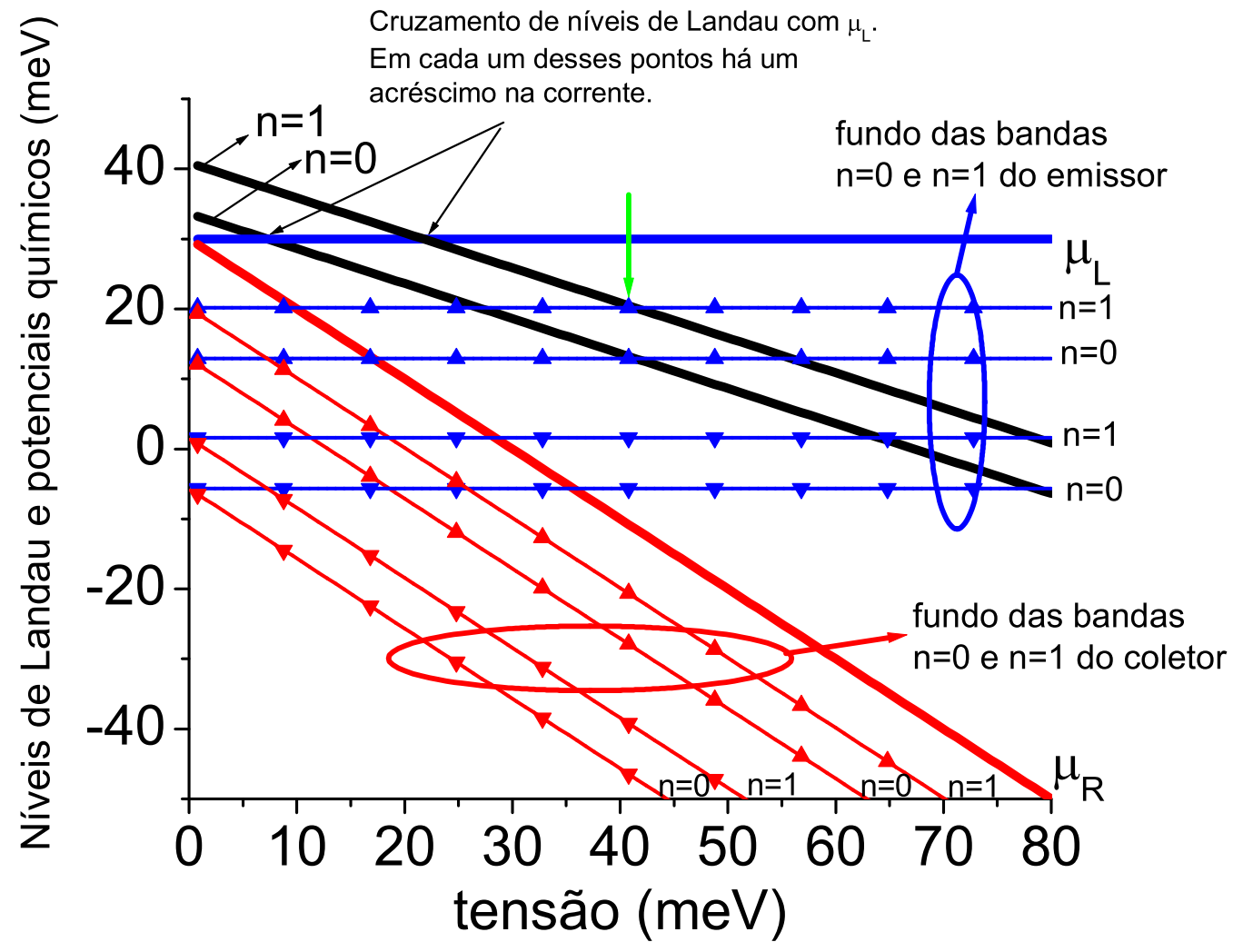

Figura 6.5: Níveis de Landau $n=0$ e $n=1$ do poço (linhas prestas), níveis de Fermi $\mu_{L}$ e $\mu_{R}$ (linhas azul e vermelha, respectivamente), e os fundos das bandas para spin $\uparrow(\Delta)$ e spin $\downarrow(\nabla)$ como função da tensão externa aplicada. Os triângulos $\Delta \mathrm{e}$ $\nabla$ indicam spins $\uparrow$ e $\downarrow$, respectivamente. Note que o fundo da banda para spin $\downarrow$ está abaixo do fundo da banda para spin $\uparrow$ em virtude do efeito Zeeman gigante. O potencial químico da esquerda $\left(\mu_{L}\right)$ é fixo, enquanto o da direita $\left(\mu_{R}\right)$ varia linearmente com $V$, $\mu_{R}=\mu_{L}-e V$. Os níveis de Landau $E_{0}$ e $E_{1}$ também seguem um comportamento linear, dado por $E_{n}=E_{n}^{0}-e V / 2$, sendo $E_{n}^{0}=\hbar \omega_{c}(n+0.5)$. O campo magnético utilizado aqui foi de $10 \mathrm{~T}$. 
Cada plateau observado na corrente e no ruído [Figs.6.4(a)-(b)] ocorre quando um novo nível de Landau entra em ressonância com os estados do emissor, isto é, fica abaixo do potencial químico $\mu_{L}$. Para discutirmos melhor esse ponto, na Fig. (6.5) ilustramos os níveis de Landau $n=0$ e $n=1$ do poço (linhas pretas) e os potenciais químicos (linhas grossas em azul e vermelho) como função de $e V$. Também indicamos os fundos das bandas de condução (linhas azuis e vermelhas com triângulos) para as sub-bandas $n=0$ e $n=1$, e as duas componentes de spin (triângulo $\triangle$ para spin $\uparrow$ e $\nabla$ para spin $\downarrow$ ).

Inicialmente $(V=0)$ os níveis de Landau encontram-se acima de $\mu_{L}$ e portanto não há corrente (observe que as linhas pretas estão inicialmente acima das linhas azuis). Para $e V \approx 8 \mathrm{meV}$ o nível $n=0$ entra em ressonância com os estados do emissor [a curva preta para $n=0$ cruza a linha azul de $\mu_{L}$ ] e a corrente começa a fluir [veja Figs.6.4(b)-(c)], atingindo assim o seu primeiro plateau. Para $e V \approx 22$ meV, o próximo nível de Landau entra em ressonância e a corrente aumenta mais, indo para o segundo plateau. Os níveis subseqüentes $n=2,3,4 \ldots$ (não ilustrados) podem gerar plateaus adicionais. Observe que para $\mathrm{eV} \approx 40 \mathrm{meV}$ os níveis $n=0$ e $n=1$ (e da mesma forma níveis maiores $n=2,3,4 \ldots$ ) cruzam o fundo da banda de condução para spins $\uparrow$ (posição indicada pela seta verde), saindo assim da ressonância com os estados do emissor, e com isso a corrente $I_{\uparrow}$ vai a zero.

Salientamos que o cruzamento dos níveis $n=0$ e $n=1$ com o fundo da banda (veja seta verde) ocorre na mesma tensão e não em tensões diferentes, como é o caso do cruzamento desses níveis com $\mu_{L}$. Por isso que a corrente $I_{\uparrow}$ vai a zero abruptamente, e não seguindo degraus. Para $e V \approx 80 \mathrm{meV}$ os níveis $n=0$ e $n=1$ cruzam os fundos das bandas para spin $\downarrow$, resultando assim num cancelamento total da corrente.

Fator de Fano. O fator de Fano $[\gamma=S /(2 e I)]$ também apresenta características contrastantes entre os casos DMS e NMS. Por exemplo, para contatos NMS temos que $\gamma$ apresenta três picos [veja setas azuis na Fig.6.4(c)] correspondentes as ressonâncias dos níveis de Landau $n=1,2,3$. No caso de terminais DMS surgem dois picos adicionais (setas verdes). Um deles corresponde ao canal $n=4$ para spins $\downarrow$, que passa a conduzir corrente em $e V \approx 65 \mathrm{meV}$. O outro pico deve-se ao fechamento dos canais para spin $\uparrow(\mathrm{em} e V=40 \mathrm{meV}$ ). Vemos então que o fator de Fano apresenta picos não só quando um nível entra em ressonância com os estados do emissor, mas também quando, pelo menos uma componente de spin, sai de ressonância.

\subsubsection{Corrente e ruído versus $B$}

Na figura (6.6) graficamos corrente e ruído como função do campo magnético, para duas voltagens diferentes $[\mathrm{eV}=30 \mathrm{meV}$-paineis da esquerda, $\mathrm{eV}=60 \mathrm{meV}$-paineis da direita], e contatos DMS e NMS, linhas sólidas e tracejadas respectivamente. 
Para $\mathrm{eV}=30 \mathrm{meV}$ e contatos DMS encontramos a corrente $I_{\uparrow}$ e o ruído $S_{\uparrow}$ aumentam inicialmente com o campo e a partir de $B \approx 2 \mathrm{~T}$ surgem oscilações devido aos níveis de Landau. A amplitude dessas oscilações intensifica-se a medida que $B$ aumenta. Para as componentes $\downarrow$ da corrente e do ruído temos inicialmente uma diminuição dos seus valores $[0<B<1.5 \mathrm{~T}]$, seguida de oscilações semelhantes as do caso $\uparrow$. O comportamento da corrente e do ruído para campos $0<B<1.5 \mathrm{~T}$ deve-se inteiramente ao desdobramento das bandas nos terminais DMS, sendo os níveis de Landau relevantes para explicar as curvas somente para $B \gtrsim 2 \mathrm{~T}$.

Para $\mathrm{eV}=60 \mathrm{meV}$ observamos um súbito aumento da corrente $I_{\downarrow}$ e do ruído $S_{\downarrow}$, seguido de uma supressão e posteriormente $(B \gtrsim 2 \mathrm{~T})$ de oscilações. Por outro lado, a componente $\uparrow$ da corrente e do ruído são totalmente suprimidas. Da mesma forma como no caso $\mathrm{eV}=30 \mathrm{meV}$, entenderemos o comportamento da corrente e do ruído para $0<B<1.5 \mathrm{~T}$ na Sec. 6.10. Seguiremos analisando diretamente as oscilações que ocorrem para $B \gtrsim 2 \mathrm{~T}$.

As oscilações observadas para $\mathrm{eV}=30 \mathrm{meV}$ e $\mathrm{eV}=60 \mathrm{meV}$ devem-se ao cruzamento dos níveis de Landau com o potencial químico $\mu_{L}$. Mais especificamente, a medida que o campo magnético aumenta, os níveis de Landau $E_{n}=\hbar \omega_{c}(n+0.5)$ crescem linearmente em energia, e eventualmente saem de ressonância com os estados do emissor, ficando acima de $\mu_{L}$. Esse efeito pode ser observado na Fig. 6.7, onde apresentamos alguns níveis de Landau (Landau "fan diagram") como função de $B$ para $e V=30 \mathrm{meV}$ e $e V=60 \mathrm{meV}$. Vemos que esses níveis vão gradualmente ficando acima de $\mu_{L}$ a medida em que o campo magnético aumenta, e conseqüentemente deixando de conduzir corrente. As linhas verticais tracejadas na Fig. 6.6 indicam o valor do campo no qual os cruzamentos entre $E_{n}$ e $\mu_{L}$ ocorrem. Em particular, na Fig. 6.6(a) indicamos os níveis de Landau $n=2,3, \ldots, 7$, e na Fig. 6.6 (b) temos $n=4,5, \ldots, 9$. Note que em cada cruzamento a corrente e o ruído diminuem, e após os cruzamentos estes voltam a crescer.

O aumento da corrente e do ruído entre os cruzamentos deve-se ao aumento da degenerescência de Landau, que permite um maior número de elétrons conduzir corrente. A diminuição da corrente e do ruído nos cruzamentos (linhas tracejadas) se dá em virtude do fechamento de um canal de condução. A queda da corrente e do ruído é tão maior quanto maior é o campo. Isso ocorre pois em campos grandes os níveis são mais degenerados, e portanto mais elétrons podem fluir por esse canal, quando esse nível sai de ressonância muito mais elétrons deixam de conduzir, e portanto a corrente e o ruído caem mais do que cairiam em campos pequenos. Esse processo cíclico de aumento e supressão da corrente e do ruído segue continuamente até que o nível de Landau $n=0$ cruza o potencial químico $\mu_{L}$, resultando em corrente e ruído nulos (esse limite não está ilustrado nas Figs.(6.6)].

Fator de Fano. Ao contrário da corrente e do ruído que aumentam entre dois cruzamentos consecutivos de níveis de Landau, o fator de Fano apresenta uma 
supressão nesse regime. Isso indica que o fluxo de elétrons tende a ficar mais correlacionado com o aumento da degenerescência de Landau. Para campos em torno das linhas tracejadas, o fator de Fano aumenta, mostrando que, quando um nível de Landau cruza $\mu_{L}$, o transporte tende a se descorrelacionar. Esse mesmo efeito foi observado experimentalmente em uma junção de tunelamento do tipo GaAs-AlAs-GaAs com pontos quânticos auto-organizados de InAs acoplados a eletrodos não magnéticos [52]. Observou-se que quando um nível de um ponto quântico se alinhava ao pontencial químico do emissor, o fator de Fano apresentava um pico, assim como observamos em nosso sistema. Muito embora seja um sistema diferente do nosso, a física é semelhante.

Um outro aspecto a se ressaltar é o fato do material DMS nos contatos levar a um aumento de $\gamma$ para $e V=30 \mathrm{meV}$ e a uma supressão de $\gamma$ para $\mathrm{eV}=$ $60 \mathrm{meV}$ à medida que o campo magnético aumenta [compare os casos DMS e NMS para ambas as tensões]. Isso mostra que a presença de material DMS altera a correlação no transporte eletrônico e esse efeito depende do valor da tensão aplicada. Podemos entender isso notando que para $\mathrm{eV}=60 \mathrm{meV}$ somente os spins $\downarrow$ conduzem corrente, isso tende a intensificar a correlação no transporte via princípio de Pauli.

Para $e V=60 \mathrm{meV}$ observamos que o fator de Fano na presença de material DMS se aproxima de 0.6 à medida que o campo aumenta. Muito embora não haja uma expressão analítica simples para o fator de Fano nesse sistema, podemos estimar o seu valor através da fórmula usual

$$
\gamma=\frac{\left(\Gamma^{L}\right)^{2}+\left(\Gamma^{R}\right)^{2}}{\left(\Gamma^{L}+\Gamma^{R}\right)^{2}}
$$

para um problema de dupla-barreira [64]. Como em nosso sistema $\Gamma_{n \sigma}^{\eta}(\epsilon)$ depende da energia, vamos adotar a seguinte estratégia: fixamos a tensão em $\mathrm{eV}=60$ meV [como na Fig.6.6(f)] e campo $B=10$ T. Fazendo o gráfico das densidades de estado para spin $\downarrow$ [Fig.6.8], podemos extrair os valores de $\Gamma_{0 \downarrow}^{L}$ e $\Gamma_{0 \downarrow}^{R}$, e em seguida substitui-los na seguinte equação

$$
\gamma=\frac{\left(\Gamma_{0 \downarrow}^{L}\right)^{2}+\left(\Gamma_{0 \downarrow}^{R}\right)^{2}}{\left(\Gamma_{0 \downarrow}^{L}+\Gamma_{0 \downarrow}^{R}\right)^{2}},
$$

que é uma adaptação da Eq.(6.36) para o nosso modelo. Observe que desprezamos a componente de spin $\uparrow$ nesse cálculo em virtude da corrente e do ruído $\uparrow$ serem desprezíveis [veja Figs.6.6(b) e (d)], não afetando assim as propriedades de transporte. Extraímos da Fig.(6.8) os valores $\Gamma_{0 \downarrow}^{L}=330 \mu \mathrm{eV}$ e $\Gamma_{0 \downarrow}^{R}=120 \mu \mathrm{eV}$, que são os valores em torno da região central do pico de $A_{0 \downarrow}(\epsilon)$. Substituindo esses dados na Eq.(6.37) encontramos

$$
\gamma=0.61
$$

que está de acordo com o resultado observado na Fig.6.6(f). Essa forma simples de se calcular $\gamma$ é tão melhor quanto mais constante são as densidades de estado 
dentro da região do pico de $A_{0 \downarrow}(\epsilon)$. Por exemplo, se uma das densidades, digamos $\Gamma_{0 \downarrow}^{L}(\epsilon)$, variar muito dentro da região do pico, não teremos um valor bem definido para $\Gamma_{0 \downarrow}^{L}(\epsilon)$, e uma integral se fará necessária para se obter resultados mais acurados. Para a configuração ilustrada na Fig.(6.8), porém, $\Gamma_{0 \downarrow}^{R}$ é essencialmente constante e $\Gamma_{0 \downarrow}^{L}$ varia pouco na região do pico $A_{0 \downarrow}$, e portanto encontramos um acordo satisfatório entre a equação (6.37) e o resultado numérico da Fig.6.6(f).

$\mathrm{Na}$ seção seguinte damos continuidade à discussão das curvas da Fig.(6.6) na faixa de campos $0<B<1.5 \mathrm{~T}$. 

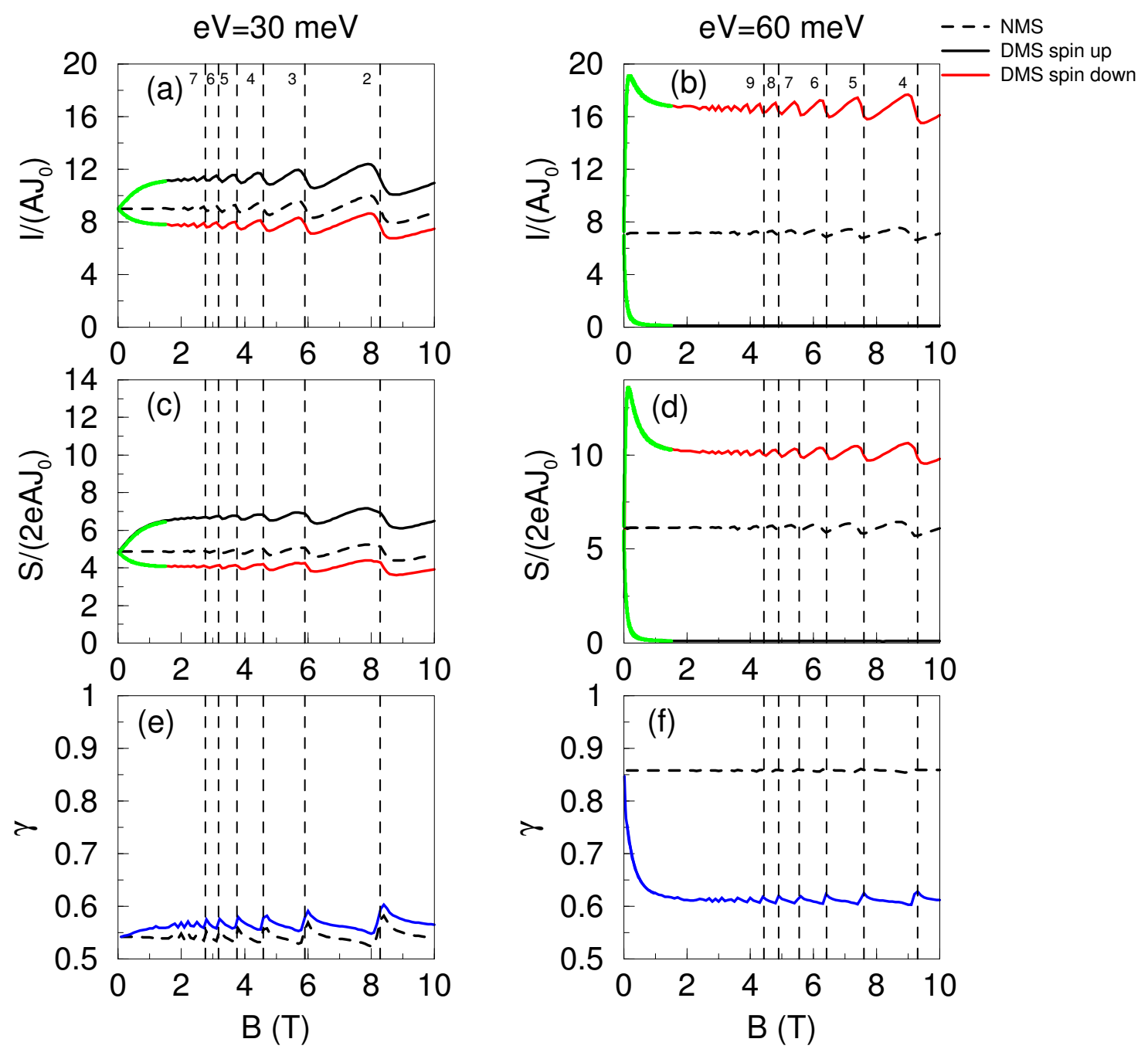

Figura 6.6: Corrente, ruído e fator de Fano como função do campo magnético para duas tensões diferentes $[e V=30 \mathrm{meV}$ e $e V=60 \mathrm{meV}$ ]. Para $e V=30 \mathrm{meV}$ [painéis da esquerda] observamos oscilações em ambas as componentes de spin, sendo que a componente $\uparrow$ é intensificada e a componentes $\downarrow$ é suprimida com relação ao caso não magnético (NMS). Essas oscilações ocorrem sempre que um nível de Landau cruza o nível de Fermi $\mu_{L}$, cruzamento este que está indicado pelas linhas verticais tracejadas. No caso $\mathrm{eV}=60 \mathrm{meV}$ temos o oposto, a componente $\downarrow$ é intensificada com o aumento do campo e a componente $\uparrow$ é suprimida na presença de campo e material DMS. A causa dessa supressão/aumento da corrente e do ruído será discutida posteriormente na Sec.6.10. O fator de Fano $\gamma=\left(S_{\uparrow}+S_{\downarrow}\right) / 2 e\left(I_{\uparrow}+I_{\downarrow}\right)$ [paineis (e) e (f)] revelam que entre dois cruzamentos consecutivos o transporte tende a se correlacionar mais, visto que o fator de Fano diminui. Exatamente no cruzamento o fator de Fano aumenta, indicando uma descorrelação no transporte quando os níveis de Landau cruzam $\mu_{L}$. As curvas verdes para $0<B<1.5 \mathrm{~T}$ correspondem ao caso sem quantização de Landau (baixos campos). Observe que essas curvas coincidem com as curvas que levam em conta os níveis de Landau. 


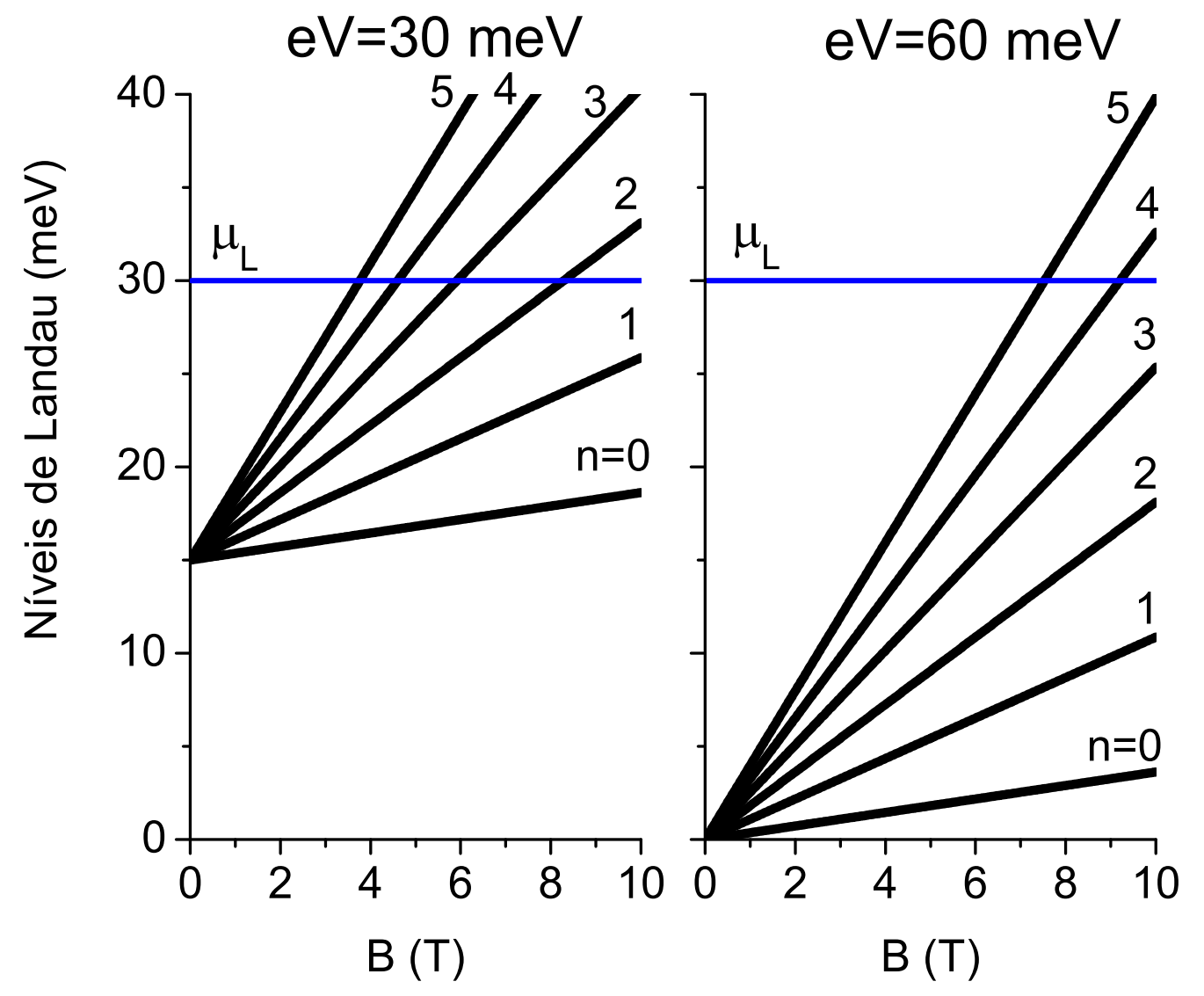

Figura 6.7: Níveis de Landau ("Landau fan diagram") como função do campo magnético $B$ para duas tensões fixas, $\mathrm{eV}=30 \mathrm{meV}$ e $\mathrm{eV}=60 \mathrm{meV}$. Os níveis de Landau variam com o campo seguindo a relação $E_{n}=\hbar \omega_{c}(n+0.5)-e V / 2$, portanto são retas cujo coeficiente angular varia com o índice de Landau. O coeficiente linear está dado pela tensão externa $e V / 2$. Para $e V=30 \mathrm{meV}$ temos até $n=2$ cruzando $\mu_{L}$ na faixa de campo graficada, enquanto que para $\mathrm{eV}=60 \mathrm{meV}$ temos o cruzamento de no máximo $n=4$ nessa faixa. Note que não levamos em conta o efeito Zeeman ordinário, que resultaria em retas para spins $\uparrow$ e $\downarrow$ de coeficientes angulares ligeiramente diferentes. Essa pequena diferença não tem nenhuma implicação adicional nas propriedades de transporte. 


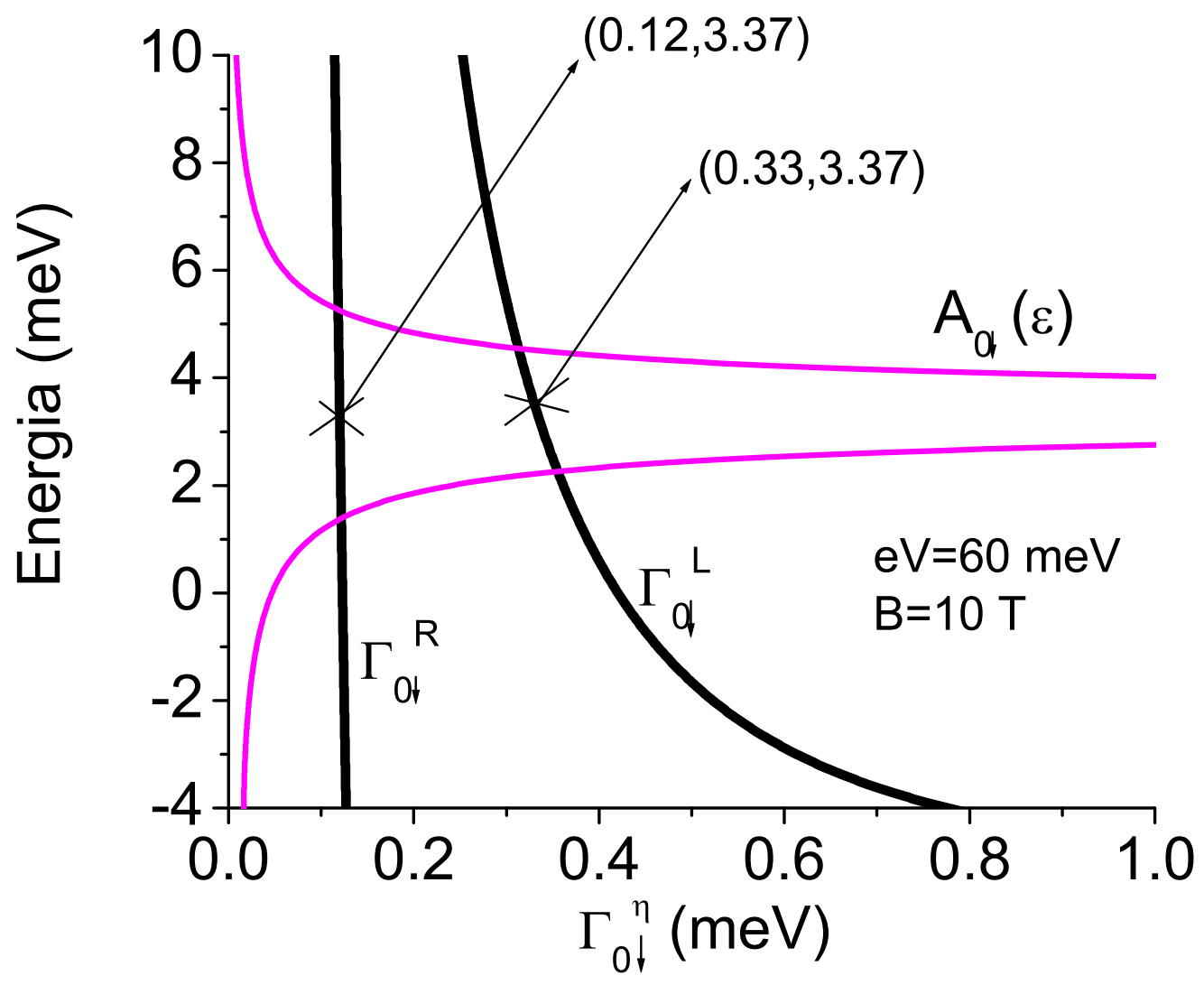

Figura 6.8: Densidade de estados dadas por $\Gamma_{0 \downarrow}^{L}$ e $\Gamma_{0 \downarrow}^{R}$ (linhas pretas) e $A_{0 \downarrow}(\epsilon)$ (linha rosa). Utilizamos os valores de $\Gamma_{0 \downarrow}^{L}$ e $\Gamma_{0 \downarrow}^{R}$ (indicados por x na figura) na expressão $\left(\Gamma_{0 \downarrow}^{L^{2}}+\Gamma_{0 \downarrow}^{R^{2}}\right) /\left(\Gamma_{0 \downarrow}^{L}+\Gamma_{0 \downarrow}^{R}\right)^{2}$ para estimar o valor do fator de Fano. 


\subsection{Regime de baixos campos $(B<1.5$ T $)$}

Ao escrevermos o Hamiltoniano (6.5) consideramos a quantização de Landau provocada pelo campo magnético. Porém, em campos pequenos, digamos $B<1.5 \mathrm{~T}$, os níveis de Landau estão tão próximos um do outro que chegam a formar um contínuo de estados. Sendo assim, poderíamos descrever os estados dos reservatórios e do poço através de um conjunto de números quânticos contínuos, $\mathbf{k}^{\eta}=\left(k_{x}^{\eta}, k_{y}^{\eta}, k_{z}^{\eta}\right)$ para o reservatório $\eta$ e $\mathbf{q}=\left(q_{x}, q_{y}\right)$ para o poço, ao contrário do que utilizamos anteriormente, $\left(k_{x}^{\eta}, m, k_{z}^{\eta}\right)$ e $\left(k_{z}, n\right)$ para os reservatórios e poço, respectivamente. Vamos considerar então o seguinte Hamiltoniano

$H=\sum_{\mathbf{k} \sigma \eta} \epsilon_{\mathbf{k}^{\eta} \sigma \eta} c_{\mathbf{k}^{\eta} \sigma \eta}^{\dagger} c_{\mathbf{k}^{\eta} \sigma \eta}+\sum_{\mathbf{q} \sigma} \epsilon_{\mathbf{q} \sigma} d_{\mathbf{q} \sigma}^{\dagger} d_{\mathbf{q} \sigma}+\sum_{\mathbf{k}^{\eta} \mathbf{q} \sigma \eta}\left(V_{\mathbf{k}^{\eta} \mathbf{q}}^{*} c_{\mathbf{k}^{\eta} \sigma \eta}^{\dagger} d_{\mathbf{q} \sigma}+V_{\mathbf{k}^{\eta} \mathbf{q}} d_{\mathbf{q} \sigma}^{\dagger} c_{\mathbf{k}^{\eta} \sigma \eta}\right)$,

onde $\mathbf{k}^{\eta}=\left(k_{x}^{\eta}, k_{y}^{\eta}, k_{z}^{\eta}\right)$ e $\mathbf{q}=\left(q_{x}, q_{y}\right)$ são conjuntos de números quânticos para o terminal $\eta(\eta=\mathrm{L}, \mathrm{R})$ e para o poço, respectivamente. Os operadores $c_{\mathbf{k}^{\eta} \sigma \eta}^{\dagger}\left(c_{\mathbf{k}^{\eta} \sigma \eta}\right)$ e $d_{\mathbf{q} \sigma}^{\dagger}\left(d_{\mathbf{q} \sigma}\right)$ criam (destroem) elétrons no eletrodo $\eta$ e no poço, respectivamente. A energia do terminal $\eta$ tem a forma

$$
\epsilon_{\mathbf{k}^{\eta} \sigma \eta}=\frac{\hbar^{2} k_{x}^{\eta^{2}}}{2 m}+\frac{\hbar^{2} k_{y}^{\eta^{2}}}{2 m}+\frac{\hbar^{2} k_{z}^{\eta^{2}}}{2 m}+\sigma \Delta+\xi_{\eta} e V
$$

onde $\sigma=+,-$ para spins $\uparrow$ e $\downarrow$, respectivamente, $\Delta$ dá o desdobramento de spin gerado pela interação de troca $s$ - $d, \Delta=\left|E_{s d}\right|=\left|-x \sigma_{z}\left\langle S_{z}\right\rangle N_{0} \alpha\right|$, e $\xi_{L}=0$ e $\xi_{R}=-1$. Para a energia do poço temos

$$
\epsilon_{q \sigma}=\epsilon_{0}+\frac{\hbar^{2} q_{x}^{2}}{2 m}+\frac{\hbar^{2} q_{y}^{2}}{2 m}
$$

onde $\epsilon_{0}=\epsilon_{00}-e V / 2$, sendo $\epsilon_{00}$ o fundo da banda do poço na ausência de tensão externa. O último termo na Eq.(6.39) dá o acoplamento entre poço e reservatórios, sendo $V_{\mathbf{k}^{\eta} \mathbf{q}}$ a matriz de acoplamento. Assumiremos que $V_{\mathbf{k}^{\eta} \mathbf{q}}$ conserva o vetor de onda paralelo, isto é, $V_{\mathbf{k}^{\eta} \mathbf{q}} \propto \delta_{k_{x}^{\eta} q_{x}} \delta_{k_{y}^{\eta} q_{y}} V$. Com isso $q_{x}$ e $q_{y}$ forma um contínuo de canais paralelos e independentes um do outro. Do ponto de vista físico, estamos desprezando espalhamentos do vetor de onda paralelo, no processo de tunelamento pelas barreiras.

Com esse Hamiltoniano podemos calcular a corrente que flui pelo sistema quando $e V \neq 0$. Usando a definição $I_{L}=-e\left\langle\dot{N}_{L}\right\rangle$, onde $N_{L}$ é o operador número $N_{L}=\sum_{\mathbf{k}^{L} \sigma} c_{\mathbf{k}^{L} \sigma L}^{\dagger} c_{\mathbf{k}^{L} \sigma L}$, podemos mostrar que

$I_{L}=\frac{e}{\hbar} \sum_{q_{x} q_{y} \sigma} \int \frac{d \epsilon}{2 \pi}\left\{\left[G_{q_{x} q_{y} \sigma}^{r}(\epsilon)-G_{q_{x} q_{y} \sigma}^{a}(\epsilon)\right] \Sigma_{q_{x} q_{y} \sigma}^{L<}(\epsilon)+G_{q_{x} q_{y} \sigma}^{<}(\epsilon)\left[\Sigma_{q_{x} q_{y} \sigma}^{L a}(\epsilon)-\Sigma_{q_{x} q_{y} \sigma}^{L r}(\epsilon)\right]\right\}$, 
onde $\Sigma_{q_{x} q_{y} \sigma}^{L r, a,<}(\epsilon)=\sum_{k_{z}^{L}}|V|^{2} g_{\mathbf{k}^{L} \sigma L}^{r, a,<}(\epsilon)$. Reescrevendo as auto-energias em termos de parte real e imaginária vem

$\Sigma_{q_{x} q_{y}}^{L r}(\epsilon)=|V|^{2} \sum_{k_{z}^{L}} g_{k^{\eta} \sigma L}(\epsilon)=|V|^{2} \sum_{k_{z}^{L}} \frac{1}{\epsilon-\epsilon\left(q_{x}, q_{y}, k_{z}^{L}\right)+i \delta}=\Lambda_{q_{x} q_{y}}^{L}(\epsilon)-\frac{i}{2} \Gamma_{q_{x} q_{y}}^{L}(\epsilon)$,

e

$$
\Sigma_{q_{x} q_{y}}^{L<}(\epsilon)=|V|^{2} \sum_{k_{z}^{L}} g_{k^{\eta} \sigma L}^{<}(\epsilon)=i n_{L}(\epsilon) \Gamma_{q_{x} q_{y}}^{L}(\epsilon),
$$

onde $\Gamma_{q_{x} q_{y}}^{L}(\epsilon)=2 \pi|V|^{2} \sum_{k_{z}^{L}} \delta\left[\epsilon-\epsilon_{\sigma L}\left(q_{x}, q_{y}, k_{z}^{L}\right)\right]$. Com isso a equação da corrente fica

$$
I_{L}=\frac{i e}{\hbar} \sum_{q_{x} q_{y} \sigma} \int \frac{d \epsilon}{2 \pi} \Gamma_{q_{x} q_{y}}^{L}(\epsilon)\left\{\left[G_{q_{x} q_{y} \sigma}^{r}(\epsilon)-G_{q_{x} q_{y} \sigma}^{a}(\epsilon)\right] n_{L}(\epsilon)+G_{q_{x} q_{y} \sigma}^{<}(\epsilon)\right\} .
$$

Observe que essa equação tem a forma usual da expressão para a corrente encontrada na literatura ${ }^{7}$, sendo que aqui os índices das funções de Green são os vetores $q_{x}$ e $q_{y}$. Como as funções de Green do poço são dadas por

$$
\begin{aligned}
G_{q_{x} q_{y}}^{r}(\epsilon) & =\frac{1}{G_{q_{x} q_{y}}^{0 r^{-1}}(\epsilon)-\Sigma_{q_{x} q_{y}}^{r}(\epsilon)} \\
G_{q_{x} q_{y}}^{<}(\epsilon) & =G_{q_{x} q_{y}}^{r}(\epsilon) \Sigma_{q_{x} q_{y}}^{<}(\epsilon) G_{q_{x} q_{y}}^{a}(\epsilon),
\end{aligned}
$$

onde $\Sigma_{q_{x} q_{y}}^{r}=\Sigma_{q_{x} q_{y}}^{L r}+\Sigma_{q_{x} q_{y}}^{R r}$ e $\Sigma_{q_{x} q_{y}}^{<}=\Sigma_{q_{x} q_{y}}^{L<}+\Sigma_{q_{x} q_{y}}^{R<}$, mostramos que

$$
G_{q_{x} q_{y}}^{r}(\epsilon)-G_{q_{x} q_{y}}^{a}(\epsilon)=\frac{-i\left[\Gamma_{q_{x} q_{y} \sigma}^{L}+\Gamma_{q_{x} q_{y} \sigma}^{R}\right]}{\left(\epsilon-\epsilon_{q_{x} q_{y}}\right)^{2}+\left(\frac{\Gamma_{q_{x} q_{y} \sigma}^{L}+\Gamma_{q_{x} q_{y} \sigma}^{R}}{2}\right)^{2}},
$$

e

$$
G_{q_{x} q_{y}}^{<}(\epsilon)=\frac{i\left[n_{L} \Gamma_{q_{x} q_{y} \sigma}^{L}+n_{R} \Gamma_{q_{x} q_{y} \sigma}^{R}\right]}{\left(\epsilon-\epsilon_{q_{x} q_{y}}\right)^{2}+\left(\frac{\Gamma_{q_{x} q_{y} \sigma}^{L}+\Gamma_{q_{x} q_{y} \sigma}^{R}}{2}\right)^{2}} .
$$

Substituindo esses resultados na equação da corrente (6.45) obtemos

$$
I_{L}=\frac{e}{\hbar} \sum_{q_{x} q_{y}} \sum_{\sigma} \int \frac{d \epsilon}{2 \pi} T_{\sigma}(\epsilon)\left[n_{L}(\epsilon)-n_{R}(\epsilon)\right]
$$

onde $T_{\sigma}(\epsilon)=\left[\Gamma_{q_{x} q_{y} \sigma}^{L} \Gamma_{q_{x} q_{y} \sigma}^{R}\right] /\left[\left(\epsilon-\epsilon_{q_{x} q_{y}}\right)^{2}+\left(\frac{\Gamma_{q_{x} q_{y} \sigma}^{L}+\Gamma_{q_{x} q_{y} \sigma}^{R}}{2}\right)^{2}\right]$. Convertendo a soma em $\left(q_{x}, q_{y}\right)$ numa integral na energia $\epsilon_{\|}=\frac{\hbar^{2} q_{\|}^{2}}{2 m}=\epsilon_{q_{x}, q_{y}}=\frac{\hbar^{2} q_{x}^{2}}{2 m}+\frac{\hbar^{2} q_{y}^{2}}{2 m}$, vem

$$
I_{L}=\frac{e A \nu_{2}}{2 \pi \hbar} \sum_{\sigma} \int d \epsilon_{\|} \int d \epsilon T_{\sigma}\left(\epsilon, \epsilon_{\|}\right)\left[n_{L}(\epsilon)-n_{R}(\epsilon)\right]
$$

onde $\nu_{2}=m / 2 \pi \hbar^{2}$ é a densidade de estados de um gás de elétrons bidimensional. Com o intuito de comparar esse resultado com outras fórmulas da literatura, passamos da integral em $\epsilon$ para uma integral em $\epsilon_{z}$. Com isso vem

$$
I_{L}=\frac{e A \nu_{2}}{2 \pi \hbar} \sum_{\sigma} \int d \epsilon_{\|} \int d \epsilon_{z} T_{\sigma}\left(\epsilon_{z}\right)\left[n_{L}^{\sigma}\left(\epsilon_{z}+\epsilon_{\|}\right)-n_{R}^{\sigma}\left(\epsilon_{z}+\epsilon_{\|}\right)\right] .
$$

\footnotetext{
${ }^{7}$ Veja por exemplo a Eq.(12.20) da referência [57].
} 
O ponto principal da equação acima é que o coeficiente de transmissão depende somente da energia longitudinal $\epsilon_{z}$. Com isso podemos efetuar facilmente a integração em $\epsilon_{\|}$. No limite de temperaturas nulas, essa integral resulta em

$$
I_{L}=\frac{e A \nu_{2}}{2 \pi \hbar} \sum_{\sigma}\left\{\int_{\epsilon_{F}^{\sigma}-e V}^{\epsilon_{F}^{\sigma}} d \epsilon_{z}\left(\epsilon_{F}^{\sigma}-\epsilon_{z}\right) T_{\sigma}\left(\epsilon_{z}\right)+e V \int_{0}^{\epsilon_{F}^{\sigma}-e V} d \epsilon_{z} T_{\sigma}\left(\epsilon_{z}\right)\right\}
$$

A equação que acabamos de obter utilizando o formalismo de Keldysh nada mais é do que a versão resolvida em spin da equação de Blanter e Büttiker ${ }^{8}$. A energia de Fermi dependente de spin é dada por $\epsilon_{F}^{\sigma}=\epsilon_{F}-\sigma \Delta$. Para o ruído também podemos mostrar que

$$
\begin{gathered}
S=\frac{e^{2} A \nu_{2}}{\pi \hbar} \sum_{\sigma}\left\{\int_{\epsilon_{F}^{\sigma}-e V}^{\epsilon_{F}^{\sigma}} d \epsilon_{z}\left(\epsilon_{F}^{\sigma}-\epsilon_{z}\right) T_{\sigma}\left(\epsilon_{z}\right)\left[1-T_{\sigma}\left(\epsilon_{z}\right)\right]+\right. \\
\left.e V \int_{0}^{\epsilon_{F}^{\sigma}-e V} d \epsilon_{z} T_{\sigma}\left(\epsilon_{z}\right)\left[1-T_{\sigma}\left(\epsilon_{z}\right)\right]\right\} .
\end{gathered}
$$

Fazendo os gráficos da corrente e do ruído obtidos pelas Eqs.(6.53) e (6.54), encontramos exatamente as curvas da Fig.(6.6) na faixa de campo que vai de zero a $1.5 \mathrm{~T}$ [veja as curvas verdes nos painéis (a)-(d) da Fig.(6.6)]. Podemos entender o comportamento da corrente e do ruído nessa faixa de campos através do coeficiente de transmissão $T\left(\epsilon_{z}\right)$, que aparece nas Eqs.(6.53) e (6.54). Como

$$
T_{\sigma}\left(\epsilon_{z}\right)=\frac{\Gamma_{\sigma}^{L}\left(\epsilon_{z}\right) \Gamma_{\sigma}^{R}\left(\epsilon_{z}\right)}{\left(\epsilon_{z}+\sigma \Delta-\epsilon_{0}\right)^{2}+\left(\frac{\Gamma_{\sigma}^{L}+\Gamma_{\sigma}^{R}}{2}\right)^{2}},
$$

graficamos na Fig.(6.9) as várias funções $\Gamma_{\sigma}^{L}\left(\epsilon_{z}\right), \Gamma_{\sigma}^{R}\left(\epsilon_{z}\right), A_{\sigma}\left(\epsilon_{z}\right)=1 /\left[\left(\epsilon_{z}+\sigma \Delta-\right.\right.$ $\left.\left.\epsilon_{0}\right)^{2}+\left(\Gamma_{\sigma}^{L}+\Gamma_{\sigma}^{R}\right)^{2} / 4\right]$, que compõem o coeficiente de transmissão $T\left(\epsilon_{z}\right)$. Observamos que com o aumento do campo, e conseqüentemente o aumento de $\Delta$, o pico de ressonância para spin $\downarrow$ se desloca para a direção de decaimento de $\Gamma_{\sigma}^{L}\left(\epsilon_{z}\right)$. Como resultado disso o coeficiente de transmissão tende a decrescer, visto que $T_{\sigma}\left(\epsilon_{z}\right)$ é dado pelo produto de $\Gamma_{\sigma}^{L}\left(\epsilon_{z}\right)$ e $A_{\sigma}\left(\epsilon_{z}\right)$. Conseqüentemente a corrente e o ruído $\downarrow$ diminuem, conforme observamos nas Figs. 6.6(a) e (c).

Já para spin $\uparrow$ temos o contrário. O aumento de $\Delta$ leva o pico de ressonância para uma região de maior $\Gamma_{\sigma}^{L}$, resultando num incremento de $T\left(\epsilon_{z}\right)$ e conseqüentemente num aumento da corrente e do ruído [Fig. 6.6(a) e (c)].

No caso $\mathrm{eV}=60 \mathrm{meV}$ [Fig.(6.10)] temos que para $\Delta=0(B=0)$ o pico de ressonância $A_{\downarrow}\left(\epsilon_{z}\right)$ está em torno da origem e se anula em $\epsilon_{z}=0$. Com o aumento de $\Delta$ esse pico afasta-se da origem levando inicialmente a um aumento do produto $\Gamma_{\downarrow}^{L} A_{\downarrow}\left(\epsilon_{z}\right)$, isto é, de $T_{\downarrow}\left(\epsilon_{z}\right)$. Aumentando mais um pouco o valor de $\Delta$, o pico $A_{\uparrow}\left(\epsilon_{z}\right)$ se desloca para regiões de menores $\Gamma_{\sigma}^{L}\left(\epsilon_{z}\right)$, resultando em uma diminuição do coeficiente de transmissão e conseqüentemente da corrente e do ruído. Para spin $\uparrow$ o aumento de $\Delta$ desloca o peso espectral $A_{\uparrow}\left(\epsilon_{z}\right)$ para uma região sem estados

\footnotetext{
${ }^{8}$ Veja Eq.79 da referência [64].
} 
no emissor (veja linha tracejada na Fig.6.9(d)], resultando assim na supressão da corrente e do ruído observada na Fig.6.6(b) e (d).

Com isso finalizamos nosso estudo sobre o sistema DMS-poço-quântico-DMS. Ressaltamos que uma variedade de resultados interessantes ainda estão por serem extraídos desse sistema e de sistemas afins, visto que a pesquisa de transporte quântico em sistema DMS está apenas em seu início. 


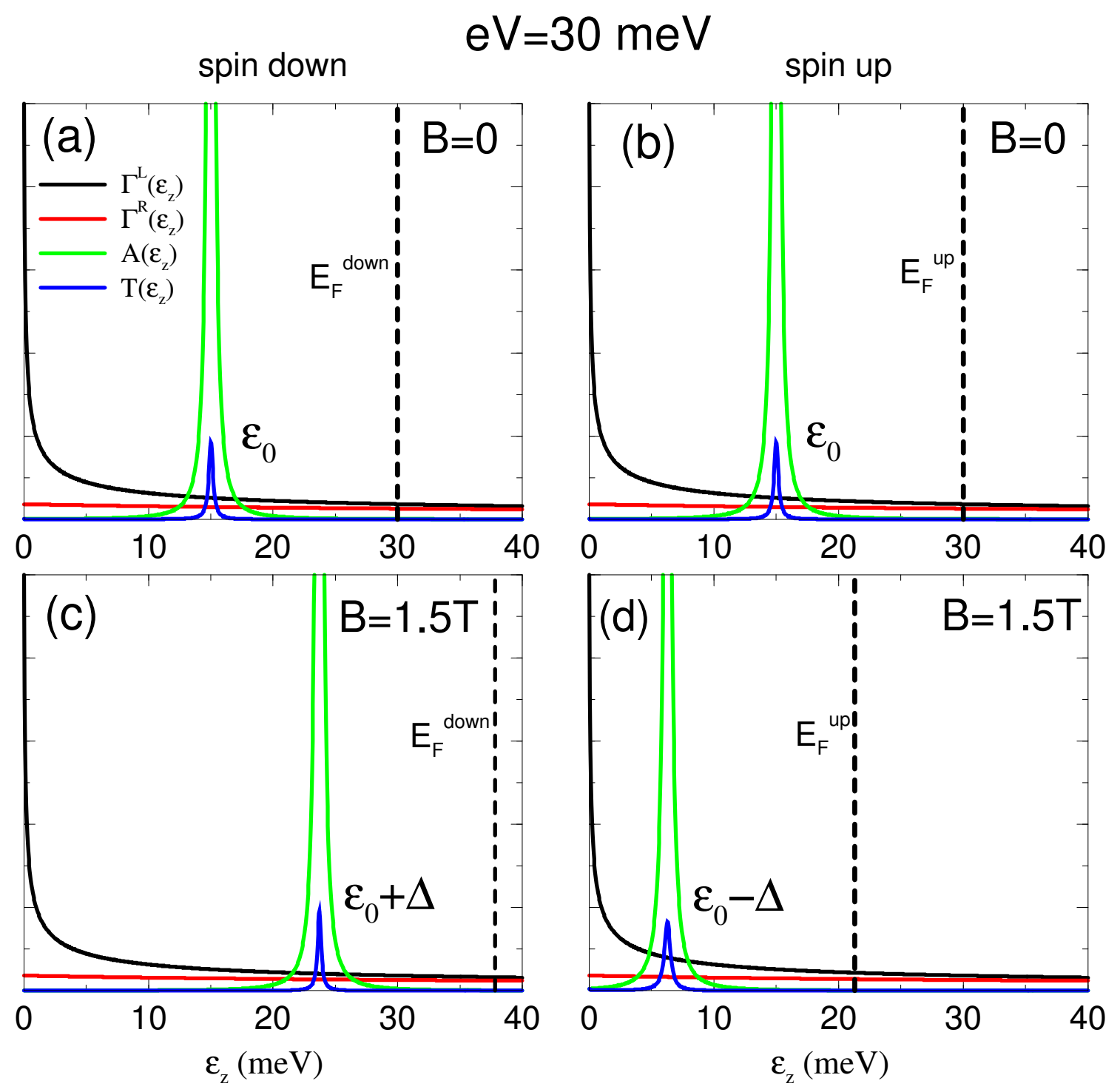

Figura 6.9: Funções $\Gamma\left(\epsilon_{z}\right)^{L}, \Gamma\left(\epsilon_{z}\right)^{R}, A_{\sigma}\left(\epsilon_{z}\right)$ e coeficiente de transmissão $T\left(\epsilon_{z}\right)$ como função da energia longitudinal $\epsilon_{z}$. Os painéis da esquerda correspondem a spin $\downarrow$ e os da direita a spin $\uparrow$. Dois valores de campo foram considerados, $B=0$ e $B=1.5 \mathrm{~T}$, o que resulta em $\Delta=0$ e $\Delta \neq 0$, respectivamente. Com o aumento do campo (ou de $\Delta$ ) temos que $A_{\downarrow}\left(\epsilon_{z}\right)$ desloca-se para uma região de menores $\Gamma^{L}\left(\epsilon_{z}\right)$ e conseqüentemente o coeficiente de transmissão $T\left(\epsilon_{z}\right)$, que é proporcional ao produto $\Gamma^{L}\left(\epsilon_{z}\right) A_{\downarrow}\left(\epsilon_{z}\right)$, diminui. Isso leva a supressão da corrente e do ruído observada na Fig. 6.6(a) e (c). Em contraste, $A_{\uparrow}\left(\epsilon_{z}\right)$ se desloca para uma região de maior $\Gamma^{L}\left(\epsilon_{z}\right)$, resultando em um acréscimo de $T_{\uparrow}\left(\epsilon_{z}\right)$ e da corrente. 


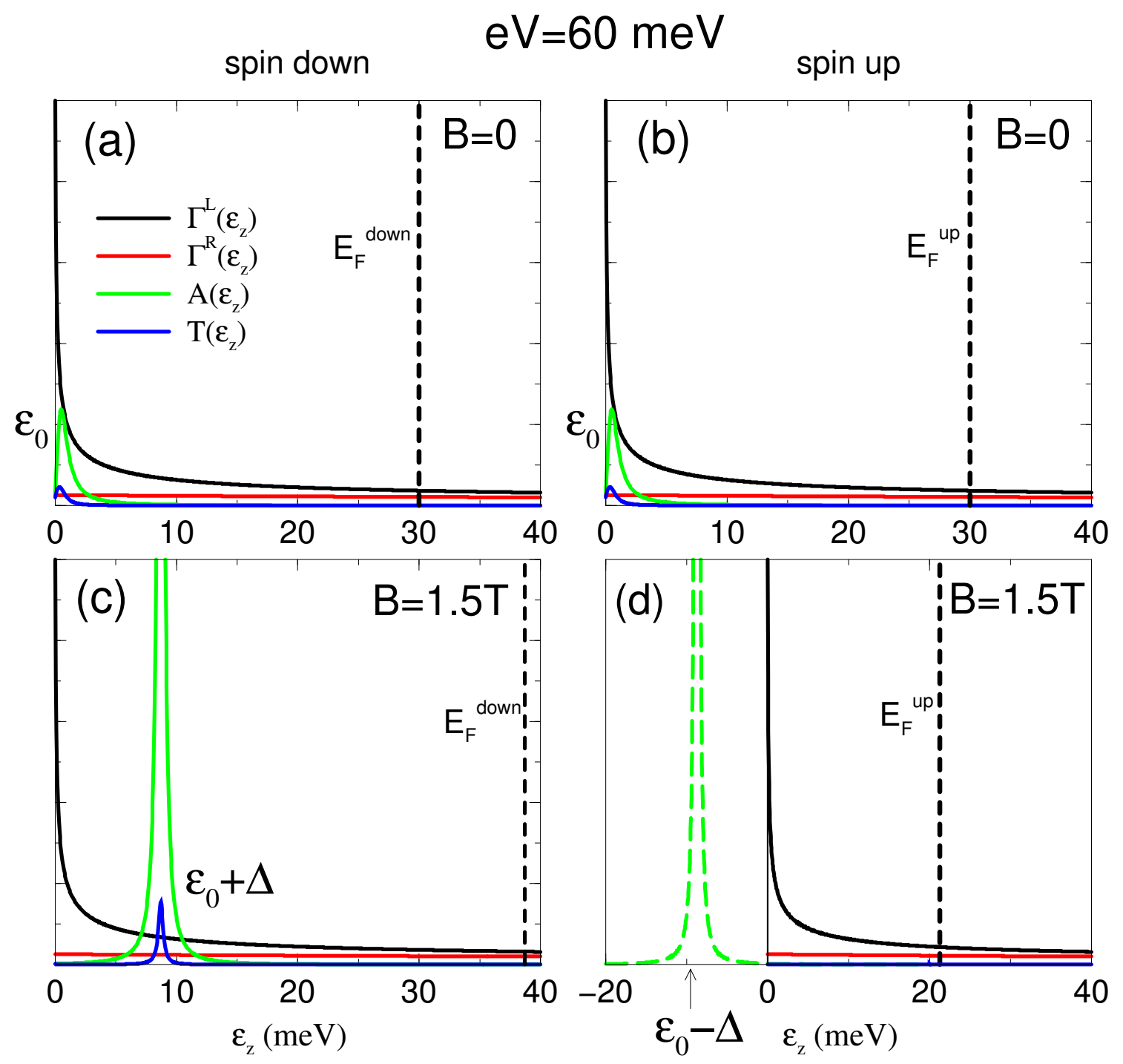

Figura 6.10: Figura semelhante a Fig. 6.9, exceto pela tensão externa adotada $(\mathrm{eV}=60$ meV). Para essa tensão o pico $A_{\sigma}\left(\epsilon_{z}\right)$ está inicialmente $(B=0, \Delta=0)$ em torno da origem. Com o aumento do campo, o pico $A_{\downarrow}\left(\epsilon_{z}\right)$ desloca-se para a direita [painel (c)], como na figura anterior, resultando num subito aumento de $T_{\downarrow}\left(\epsilon_{z}\right)$, seguido de uma supressão a medida que $A_{\downarrow}\left(\epsilon_{z}\right)$ se desloca para regiões de $\Gamma^{L}\left(\epsilon_{z}\right)$ menores. Já o pico $A_{\uparrow}\left(\epsilon_{z}\right)$ se desloca para a esquerda com o aumento de $B$ [veja linha tracejada no painel (d)], indo para uma região na qual não há estados eletrônicos. Como resultado disso obtemos coeficiente de transmissão nulo, e a corrente e o ruído são suprimidos como vemos na Fig. 6.6(b) e (d). 


\section{Capítulo 7}

\section{Conclusão}

Com o desenvolvimento da eletrônica de spin, uma variedade de novos efeitos e dispositivos deverão surgir nos próximos anos, como a MRAM (magnetic randomaccess memory) que já se encontra num estágio avançado de implementação, o spin-FET (field effect transistor) e microchips quânticos. Essa nova eletrônica deverá revolucionar a microeletrônica convencional (chips de silício), com dispositivos menores, mais versáteis e mais velozes. Estima-se que a eletrônica de spin movimentará um novo mercado multibilionário [21].

O estudo do transporte quântico dependente de spin em sistemas nanoscópicos, operando a nível de single electrons (e.g. ponto quântico), é fundamental para a compreensão e previsão de novos efeitos que combinem a carga e o spin dos portadores. Vale salientar que sistemas desse tipo poderão servir de base para a implementação da eletrônica de spin em estado sólido [23]. Em particular, estudar corrente e ruído dependentes de spin, poderá fornecer informações físicas relevantes no que diz respeito a natureza das correlações envolvidas em cada sistema, como correlações de Pauli e de Coulomb. Além de dizer como essas correlações influenciam no transporte.

O primeiro passo de nosso estudo consistiu na derivação de equações para a corrente e para o ruído utilizando o formalismo de funções de Green de nãoequilíbrio. Procuramos apresentar uma maneira sistemática de se calcular ruído (térmico+shot noise) através de definições de funções de Green de mais alta ordem e de expansões da matriz $S$. Quando possível, fizemos a conexão de nossas expressões com fórmulas-padrão da literatura (e.g. Landauer-Büttiker).

Em seguida aplicamos nossa formulação para estudar três sistemas dependentes de spin: (1) FM-PQ-FM, (2) ponto quântico acoplado a múltiplos terminais ferromagnéticos, (3) DMS-poço-DMS. Para cada um desses sistemas derivamos, através do formalismo de funções de Green de não-equilíbrio, equações para a corrente e para o ruído dependentes de $\operatorname{spin}^{1}$. Seguimos apresentando um sumário dos prin-

\footnotetext{
${ }^{1}$ Com exceção do sistema composto por um ponto quântico acoplado a multiplos terminais ferromagnéticos, em que nos limitamos ao estudo da corrente.
} 
cipais achados.

Sistema FM-PQ-FM. Através da formulação desenvolvida em nosso estudo, analisamos a corrente e o ruído em um sistema de ponto quântico acoplado a terminais ferromagnéticos. As configurações paralela (P) e anti-paralela (AP), entre as magnetizações dos eletrodos, foram consideradas. Observamos que a polarização dos terminais e os seus alinhamentos magnéticos, combinados com espalhamento de spin e interação de Coulomb, leva a uma série de efeitos dependentes de spin. Dentre esses efeitos, destacamos o bloqueio de Coulomb dependente de spin, que ocorre quando o ponto quântico tem uma magnetização $\left(m=n_{\uparrow}-n_{\downarrow}\right)$ não nula. Esse bloqueio de Coulomb dependente de spin leva a uma polarização da corrente que pode ser ajustada através da voltagem externa.

Os efeitos do bloqueio de Coulomb dependente de spin sobre o ruído também foram discutidos. Encontramos que uma das componentes $S_{\uparrow}$ ou $S_{\downarrow}$ do ruído pode ser mais ou menos suprimida com relação a outra em virtude da "polarização" do bloqueio de Coulomb (o mesmo efeito se manifesta nas componentes $I_{\uparrow}$ e $I_{\downarrow}$ da corrente).

A dependência da corrente e do ruído (mais especificamente do shot noise) com o espalhamento de spin também foi analisada. Encontramos que para a configuração AP, o espalhamento de spin (com intensidade $R$ ) tende a suprimir o shot-noise e aumentar a corrente para uma certa faixa de valores de $R$. Portanto corrente e ruído apresentam comportamentos contrastantes como função de $R$. Na configuração paralela notamos que corrente e ruído são insensíveis ao espalhamento de spin. Através do fator de Fano, conseguimos interpretar a supressão do ruído em termos de uma intensificação da correlação de Pauli, que se deve ao espalhamento de spin.

Também notamos que o espalhamento de spin leva a um aumento do ruído térmico quando o sistema está alinhado anti-paralelamente. Já o ruído térmico na configuração paralela é insensível ao espalhamento de spin. Um outro efeito interessante é a possibilidade de se controlar o ruído térmico através de uma tensão de porta. Mostramos que variando a posição do nível $\epsilon_{d}$ do ponto quântico, podemos aumentar ou suprimir o ruído térmico, que atinge seu máximo quando $\epsilon_{d}$ se alinha aos potenciais químicos dos reservatórios. Esse efeito é observado nas duas configurações magnéticas P e AP.

Os efeitos da interação de Coulomb e do espalhamento de spin sobre a magneto resistência de tunelamento (TMR) também foram analisados. Encontramos que a interação de Coulomb tende a aumentar a TMR no regime de bloqueio de Coulomb no caso de $R=0$. Já o espalhamento de spin tende a suprimir o sinal de TMR. Em particular, o efeito combinado da interação de Coulomb e do espalhamento de spin resulta numa supressão adicional da TMR.

Sistema de um ponto quântico acoplado a múltiplos terminais. Para esse sistema mostramos ser possível gerar corrente com polarizações $\uparrow$ e $\downarrow$ simultane- 
amente em eletrodos diferentes, a partir de uma corrente $\uparrow$-polarizada. Mais especificamente, encontramos que uma corrente pode sair $\uparrow$-polarizada do terminal emissor, entrar $\downarrow$-polarizada em um terminal coletor e $\uparrow$-polarizada em um outro terminal coletor. Portanto esse sistema opera simultaneamente como um amplificador e um inversor de polarização de corrente. Esse tipo de efeito é obtido variando-se o alinhamento ferromagnético dos eletrodos e suas polarizações. Três configurações magnéticas foram analisadas: (1) todos os reservatórios alinhados paralelamente entre si, (2) os dois reservatórios coletores alinhados antiparalelamente com relação ao emissor, e (3) os reservatórios coletores alinhados anti-paralelamente entre si. Os efeitos de amplificação e inversão das polarizações das corrente são obtidas nos casos (2) e (3), variando-se a polarização dos eletrodos.

Sistema DMS-poço-DMS. Por último, calculamos corrente e ruído em um sistema de poço quântico acoplado a barreiras de tunelamento. Derivamos, via funções de Green, equações para a corrente e o ruído que levam em conta a interação de troca $s$ - $d$ nos contatos magnéticos e quantização de Landau. Observamos uma dependência da polarização da corrente como função da tensão externa, e oscilações na corrente, no ruído e no fator de Fano como função do campo magnético. Também estudamos os efeitos da tensão externa sobre a largura de linha dos níveis de Landau do poço.

Perspectivas futuras. Certamente muitos resultados físicos interessantes ainda estão por serem extraídos dos sistemas estudados aqui. Devido a sua riqueza física, torna-se difícil obter todos os aspectos e regimes possíveis. Uma grande variedade de resultados interessantes sobre o sistema FM-PQ-FM tem sido publicado na literatura recente [12],[33],[56],[60]. Sistemas com múltiplos terminais também começam a ser analisados [96]-[98], e do mesmo modo sistemas de dupla barreira com DMS [61], [62]. Esperamos que os resultados apresentados aqui tenham contribuído com a pesquisa nesses sistemas e venham a motivar ainda mais novos estudos.

Uma seqüência natural de estudo a partir daqui, seria a inclusão de interação de Coulomb e espalhamento de spin no sistema de múltiplos terminais acoplados a um ponto quântico, e também o cálculo do ruído nesse sistema. Do mesmo modo, a inclusão de interações como elétron-elétron, elétron-fônons no sistema DMS-poço-DMS pode resultar em novos efeitos.

Além disso, a equação para o ruído que deduzimos aqui [Eq.(3.43)] [58], tem se mostrado apropriada para estudar outros sistemas, como um ponto quântico, com um modo de fônon, acoplado a terminais [99]. Com isso vemos a possibilidade de no futuro, abordarmos sistemas diversos dos estudados aqui. Uma possibilidade seria o cálculo do ruído em pontos quânticos no regime de quantum shuttle [100], levando em conta terminais ferromagnéticos, e conseqüentemente efeitos dependentes de spin. Uma outra possibilidade seria um arranjo de dois pontos quânticos acoplados entre si e a eletrodos ferromagnéticos [101]. 
Uma variedade de outros sistemas ainda poderiam ser citados, porém nos limitaremos a esses. Apesar da spintrônica estar na sua fase inicial, tendo muitos desafios a serem superados pela frente, acreditamos que nas próximas décadas veremos o surgimento de novos dispositivos baseados no spin dos portadores. Intensas pesquisas vem sendo desenvolvidas atualmente nessa área e já se vê progressos em muitos aspectos. Por exemplo, no que diz respeito a injeção de spins de um metal ferromagnético em um semicondutor [102], ou de um semicondutor magnético em um semicondutor normal [103]. Esperamos que esse trabalho tenha contribuído para o desenvolvimento da spintrônica, e motivado ainda mais a pesquisa em sistemas dependentes de spin. 


\section{Apêndice A}

\section{Cálculo da função de Green $G_{i \mathbf{k} \sigma \eta}^{<}(t, t)$}

Nesse apêndice derivamos uma expressão para a função de Green $G_{i \mathbf{k} \sigma \eta}^{<}(t, t)$, que aparece na equação da corrente [Eq.3.13]. O procedimento descrito aqui foi originalmente proposto por A. P. Jauho et al. [7]. Nesse procedimento, calcula-se uma equação para $G_{i \mathbf{k} \sigma \eta}\left(\tau, \tau^{\prime}\right)=-i\left\langle T_{C} d_{i}(\tau) c_{\mathbf{k} \sigma \eta}^{\dagger}\left(\tau^{\prime}\right)\right\rangle$ através da expansão da matriz $S$, e em seguida se efetua continuação analítica.

Comecemos reescrevendo $G_{i \mathbf{k} \sigma \eta}\left(\tau, \tau^{\prime}\right)$ na representação de interação com relação a $H_{T}$. Com isso vem

$$
G_{i \mathbf{k} \sigma \eta}\left(\tau, \tau^{\prime}\right)=-i\left\langle T_{C} S \widetilde{d}_{i}(\tau) \widetilde{c}_{\mathbf{k} \sigma \eta}^{\dagger}\left(\tau^{\prime}\right)\right\rangle,
$$

onde $S=T_{C} e^{-i \int_{C} d \tau_{1} \widetilde{H}_{T}\left(\tau_{1}\right)}$. O símbolo tio indica que os operadores estão na representação de interação. Expandindo $S$ temos

$$
S=\sum_{n=0}^{\infty} \frac{(-i)^{n}}{n !} \int_{C} d \tau_{1} \int_{C} d \tau_{2} \cdots \int_{C} d \tau_{n} T_{C} \widetilde{H}_{T}\left(\tau_{1}\right) \widetilde{H}_{T}\left(\tau_{2}\right) \cdots \widetilde{H}_{T}\left(\tau_{n}\right)
$$

Sendo

$$
\widetilde{H}_{T}\left(\tau_{i}\right)=\sum_{\mathbf{k}_{i} \sigma_{i} \eta_{i} i_{i}} \frac{(-1)^{i_{i} \delta_{\sigma_{i} \downarrow}}}{\sqrt{2}}\left[t_{\mathbf{k}_{i} \sigma_{i}}^{*} \widetilde{d}_{i_{i}}^{\dagger}\left(\tau_{i}\right) \widetilde{c}_{\mathbf{k}_{i} \sigma_{i} \eta_{i}}\left(\tau_{i}\right)+t_{\mathbf{k}_{i} \sigma_{i}} \widetilde{c}_{\mathbf{k}_{i} \sigma_{i} \eta_{i}}^{\dagger}\left(\tau_{i}\right) \widetilde{d}_{i_{i}}\left(\tau_{i}\right)\right],
$$

vem

$$
\begin{aligned}
S= & \sum_{n=0}^{\infty} \frac{(-i)^{n}}{n !} \int_{C} d \tau_{1} \int_{C} d \tau_{2} \cdots \int_{C} d \tau_{n} T_{C} \\
& \sum_{\mathbf{k}_{1} \sigma_{1} \eta_{1} i_{1}} \frac{(-1)^{i_{1} \delta_{\sigma_{1} \downarrow} \downarrow}}{\sqrt{2}}\left[t_{\mathbf{k}_{1} \sigma_{1}}^{*} \widetilde{d}_{i_{1}}^{\dagger}\left(\tau_{1}\right) \widetilde{c}_{\mathbf{k}_{1} \sigma_{1} \eta_{1}}\left(\tau_{1}\right)+t_{\mathbf{k}_{1} \sigma_{1}} \widetilde{c}_{\mathbf{k}_{1} \sigma_{1} \eta_{1}}^{\dagger}\left(\tau_{1}\right) \widetilde{d}_{i_{1}}\left(\tau_{1}\right)\right] \times \\
& \sum_{\mathbf{k}_{2} \sigma_{2} \eta_{2} i_{2}} \frac{(-1)^{i_{2} \delta_{\sigma_{2} \downarrow} \downarrow}}{\sqrt{2}}\left[t_{\mathbf{k}_{2} \sigma_{2}}^{*} \widetilde{d}_{i_{2}}^{\dagger}\left(\tau_{2}\right) \widetilde{c}_{\mathbf{k}_{2} \sigma_{2} \eta_{2}}\left(\tau_{2}\right)+t_{\mathbf{k}_{2} \sigma_{2}} \widetilde{c}_{\mathbf{k}_{2} \sigma_{2} \eta_{2}}^{\dagger}\left(\tau_{2}\right) \widetilde{d}_{i_{2}}\left(\tau_{2}\right)\right] \times \\
& \sum_{\mathbf{k}_{n} \sigma_{n} \eta_{n} i_{n}} \frac{(-1)^{i_{n} \delta_{\sigma_{n} \downarrow} \downarrow}}{\sqrt{2}}\left[t_{\mathbf{k}_{n} \sigma_{n}}^{*} \widetilde{d}_{i_{n}}^{\dagger}\left(\tau_{n}\right) \widetilde{c}_{\mathbf{k}_{n} \sigma_{n} \eta_{n}}\left(\tau_{n}\right)+t_{\mathbf{k}_{n} \sigma_{n}} \widetilde{c}_{\mathbf{k}_{n} \sigma_{n} \eta_{n}}^{\dagger}\left(\tau_{n}\right) \widetilde{d}_{i_{n}}\left(\tau_{n}\right)\right] .
\end{aligned}
$$


Substituindo essa expansão na função de Green (A.1) e agrupando termos (via teorema de Wick) obtemos

$$
\begin{aligned}
G_{i \mathbf{k} \sigma \eta}\left(\tau, \tau^{\prime}\right)= & -i \int_{C} d \tau_{1} \sum_{\mathbf{k}_{1} \sigma_{1} \eta_{1} i_{1}} \frac{(-1)^{i_{1} \delta_{\sigma_{1} \downarrow}}}{\sqrt{2}} t_{\mathbf{k}_{1} \sigma_{1}}^{*} \sum_{n=1}^{\infty} \frac{(-i)^{n}}{n !} n \times \\
& \left\{\int_{C} d \widetilde{c}_{\mathbf{k}_{1} \sigma_{1} \eta_{1}}\left(\tau_{1}\right) \widetilde{c}_{\mathbf{k} \sigma \eta}^{\dagger}\left(\tau^{\prime}\right)\right\rangle\left\langle T_{C} \widetilde{d}_{i}(\tau) \widetilde{d}_{i_{1}}^{\dagger}\left(\tau_{1}\right) \times\right. \\
& \frac{(-1)^{i_{2} \delta_{2} \downarrow}}{\sqrt{2}} \times \\
& \ldots \quad\left[t_{\mathbf{k}_{2} \sigma_{2} \eta_{2} i_{2}}^{*} \widetilde{d}_{i_{2}}^{\dagger}\left(\tau_{2}\right) \widetilde{c}_{\mathbf{k}_{2} \sigma_{2} \eta_{2}}\left(\tau_{2}\right)+t_{\mathbf{k}_{2} \sigma_{2}} \widetilde{c}_{\mathbf{k}_{2} \sigma_{2} \eta_{2}}^{\dagger}\left(\tau_{2}\right) \widetilde{d}_{i_{2}}\left(\tau_{2}\right)\right] \times \\
& \left.\left.\sum_{\mathbf{k}_{n} \sigma_{n} \eta_{n} i_{n}} \frac{(-1)^{i_{n} \delta_{\sigma_{n} \downarrow} \downarrow}}{\sqrt{2}}\left[t_{\mathbf{k}_{n} \sigma_{n}}^{*} \widetilde{d}_{i_{n}}^{\dagger}\left(\tau_{n}\right) \widetilde{c}_{\mathbf{k}_{n} \sigma_{n} \eta_{n}}\left(\tau_{n}\right)+t_{\mathbf{k}_{n} \sigma_{n}} \widetilde{c}_{\mathbf{k}_{n} \sigma_{n} \eta_{n}}^{\dagger}\left(\tau_{n}\right) \widetilde{d}_{i_{n}}\left(\tau_{n}\right)\right]\right\rangle\right\} .
\end{aligned}
$$

Reescrevendo essa equação vem

$$
\begin{aligned}
G_{i \mathbf{k} \sigma \eta}\left(\tau, \tau^{\prime}\right)= & -i \int_{C} d \tau_{1} \sum_{\mathbf{k}_{1} \sigma_{1} \eta_{1} i_{1}} \frac{(-1)^{i_{1} \delta_{\sigma_{1} \downarrow}}}{\sqrt{2}} t_{\mathbf{k}_{1} \sigma_{1}}^{*}(-i)\left\langle T_{C} \widetilde{c}_{\mathbf{k}_{1} \sigma_{1} \eta_{1}}\left(\tau_{1}\right) \widetilde{c}_{\mathbf{k} \sigma \eta}^{\dagger}\left(\tau^{\prime}\right) \times\right\rangle \\
& \left\langleT _ { C } \widetilde { d } _ { i } ( \tau ) \widetilde { d } _ { i _ { 1 } } ^ { \dagger } ( \tau _ { 1 } ) \left\{\sum_{n=1}^{\infty} \frac{(-i)^{(n-1)}}{(n-1) !} \int_{C} d \tau_{2} \sum_{\mathbf{k}_{2} \sigma_{2} \eta_{2} i_{2}} \frac{(-1)^{i_{2} \delta_{\sigma_{2} \downarrow}}}{\sqrt{2}} \times\right.\right. \\
& {\left[t_{\mathbf{k}_{2} \sigma_{2}}^{*} \widetilde{d}_{i_{2}}^{\dagger}\left(\tau_{2}\right) \widetilde{c}_{\mathbf{k}_{2} \sigma_{2} \eta_{2}}\left(\tau_{2}\right)+t_{\mathbf{k}_{2} \sigma_{2}} \widetilde{c}_{\mathbf{k}_{2} \sigma_{2} \eta_{2}}^{\dagger}\left(\tau_{2}\right) \widetilde{d}_{i_{2}}\left(\tau_{2}\right)\right] \times } \\
& \ldots \\
& \left.\left.\sum_{\mathbf{k}_{n} \sigma_{n} \eta_{n} i_{n}} \frac{(-1)^{i_{n} \delta_{\sigma_{n} \downarrow} \downarrow}}{\sqrt{2}}\left[t_{\mathbf{k}_{n} \sigma_{n}}^{*} \widetilde{d}_{i_{n}}^{\dagger}\left(\tau_{n}\right) \widetilde{c}_{\mathbf{k}_{n} \sigma_{n} \eta_{n}}\left(\tau_{n}\right)+t_{\mathbf{k}_{n} \sigma_{n}} \widetilde{c}_{\mathbf{k}_{n} \sigma_{n} \eta_{n}}^{\dagger}\left(\tau_{n}\right) \widetilde{d}_{i_{n}}\left(\tau_{n}\right)\right]\right\}\right\rangle .
\end{aligned}
$$

Agora note que o termo entre chaves é novamente a matriz $S$, isto é,

$$
\begin{aligned}
S= & \sum_{n=1}^{\infty} \frac{(-i)^{(n-1)}}{(n-1) !} \int_{C} d \tau_{2} \sum_{\mathbf{k}_{2} \sigma_{2} \eta_{2} i_{2}} \frac{(-1)^{i_{2} \delta_{\sigma_{2} \downarrow}}}{\sqrt{2}} \times \\
& {\left[t_{\mathbf{k}_{2} \sigma_{2}}^{*} \widetilde{d}_{i_{2}}^{\dagger}\left(\tau_{2}\right) \widetilde{c}_{\mathbf{k}_{2} \sigma_{2} \eta_{2}}\left(\tau_{2}\right)+t_{\mathbf{k}_{2} \sigma_{2}} \widetilde{c}_{\mathbf{k}_{2} \sigma_{2} \eta_{2}}^{\dagger}\left(\tau_{2}\right) \widetilde{d}_{i_{2}}\left(\tau_{2}\right)\right] \times } \\
& \quad \cdots \\
& \left.\sum_{\mathbf{k}_{n} \sigma_{n} \eta_{n} i_{n}} \frac{(-1)^{i_{n} \delta_{\sigma_{n} \downarrow}}}{\sqrt{2}}\left[t_{\mathbf{k}_{n} \sigma_{n}}^{*} \widetilde{d}_{i_{n}}^{\dagger}\left(\tau_{n}\right) \widetilde{c}_{\mathbf{k}_{n} \sigma_{n} \eta_{n}}\left(\tau_{n}\right)+t_{\mathbf{k}_{n} \sigma_{n}} \widetilde{c}_{\mathbf{k}_{n} \sigma_{n} \eta_{n}}^{\dagger}\left(\tau_{n}\right) \widetilde{d}_{i_{n}}\left(\tau_{n}\right)\right]\right\rangle .
\end{aligned}
$$

Com isso, a Eq.(A.5) fica

$$
\begin{aligned}
& G_{i \mathbf{k} \sigma \eta}\left(\tau, \tau^{\prime}\right)=-i \int_{C} d \tau_{1} \sum_{\mathbf{k}_{1} \sigma_{1} \eta_{1} i_{1}} \frac{(-1)^{i_{1} \delta_{\sigma_{1} \downarrow}}}{\sqrt{2}} t_{\mathbf{k}_{1} \sigma_{1}}^{*}(-i) \times \\
&\left\langle T_{C} \widetilde{c}_{\mathbf{k}_{1} \sigma_{1} \eta_{1}}\left(\tau_{1}\right) \widetilde{c}_{\mathbf{k} \sigma \eta}^{\dagger}\left(\tau^{\prime}\right)\right\rangle\left\langle T_{C} \widetilde{d}_{i}(\tau) \widetilde{d}_{i_{1}}^{\dagger}\left(\tau_{1}\right) S\right\rangle \\
&=-i \int_{C} d \tau_{1} \sum_{\mathbf{k}_{1} \sigma_{1} \eta_{1} i_{1}} \frac{(-1)^{i_{1} \delta_{\sigma_{1} \downarrow}}}{\sqrt{2}} t_{\mathbf{k}_{1} \sigma_{1}}^{*}(-i) \times \\
&\left\langle T_{C} \widetilde{c}_{\mathbf{k}_{1} \sigma_{1} \eta_{1}}\left(\tau_{1}\right) \widetilde{c}_{\mathbf{k} \sigma \eta}^{\dagger}\left(\tau^{\prime}\right)\right\rangle\left\langle T_{C} d_{i}(\tau) d_{i_{1}}^{\dagger}\left(\tau_{1}\right)\right\rangle .
\end{aligned}
$$


Observe que os operadores na média $\left\langle\widetilde{c}_{\mathbf{k}_{1} \sigma_{1} \eta_{1}}\left(\tau_{1}\right) \widetilde{c}_{\mathbf{k} \sigma \eta}^{\dagger}\left(\tau^{\prime}\right)\right\rangle$ permaneceram na representação de interação, isto é, sem o acoplamento $H_{T}$, enquanto os operadores em $\left\langle T_{C} d_{i}(\tau) d_{i_{1}}^{\dagger}\left(\tau_{1}\right)\right\rangle$ voltaram para a representação de Heisenberg. Sendo assim, a primeira média corresponde a uma função de Green não-interagente, enquanto a segunda média contém interações (função de Green interagente). Como $\left\langle\widetilde{c}_{\mathbf{k}_{1} \sigma_{1} \eta_{1}}\left(\tau_{1}\right) \widetilde{c}_{\mathbf{k} \sigma \eta}^{\dagger}\left(\tau^{\prime}\right)\right\rangle \propto \delta_{\mathbf{k k}_{1}} \delta_{\sigma \sigma_{1}} \delta_{\eta \eta_{1}}$, obtemos

$G_{i \mathbf{k} \sigma \eta}\left(\tau, \tau^{\prime}\right)=-i \int_{C} d \tau_{1} \sum_{i_{1}} \frac{(-1)^{i_{1} \delta_{\sigma \downarrow}}}{\sqrt{2}} t_{\mathbf{k} \sigma}^{*}(-i)\left\langle T_{C} \widetilde{c}_{\mathbf{k} \sigma \eta}\left(\tau_{1}\right) \widetilde{c}_{\mathbf{k} \sigma \eta}^{\dagger}\left(\tau^{\prime}\right)\right\rangle\left\langle T_{C} d_{i}(\tau) d_{i_{1}}^{\dagger}\left(\tau_{1}\right)\right\rangle$.

Definindo a função de Green do ponto quântico $G_{i i_{1}}\left(\tau, \tau_{1}\right)=-i\left\langle T_{C} d_{i}(\tau) d_{i_{1}}^{\dagger}\left(\tau_{1}\right)\right\rangle$ e dos terminais $g_{\mathbf{k} \sigma \eta}\left(\tau_{1}, \tau^{\prime}\right)=-i\left\langle T_{C} \widetilde{c}_{\mathbf{k} \sigma \eta}\left(\tau_{1}\right) \widetilde{c}_{\mathbf{k} \sigma \eta}^{\dagger}\left(\tau^{\prime}\right)\right\rangle$, podemos reescrever (A.7) em termos dessas funções de Green,

$$
G_{i \mathbf{k} \sigma \eta}\left(\tau, \tau^{\prime}\right)=\sum_{i_{1}} \int_{C} d \tau_{1} t_{\mathbf{k} \sigma}^{*} \frac{(-1)^{i_{1} \delta_{\sigma \downarrow}}}{\sqrt{2}} G_{i i_{1}}\left(\tau, \tau_{1}\right) g_{\mathbf{k} \sigma \eta}\left(\tau_{1}, \tau^{\prime}\right) .
$$

Salientamos que essa mesma equação pode ser obtida de uma forma alternativa, através do método de equação de movimento. Aplicamos agora a Eq.(2.29) discutida no capítulo 2, para obter a função de Green menor $G_{i \mathbf{k} \sigma \eta}^{<}\left(t, t^{\prime}\right)$. Com isso vem

$$
G_{i \mathbf{k} \sigma \eta}^{<}\left(t, t^{\prime}\right)=\sum_{i_{1}} t_{\mathbf{k} \sigma}^{*} \frac{(-1)^{i_{1} \delta_{\sigma \downarrow}}}{\sqrt{2}} \int d t_{1}\left[G_{i i_{1}}^{r}\left(t, t_{1}\right) g_{\mathbf{k} \sigma \eta}^{<}\left(t_{1}, t^{\prime}\right)+G_{i i_{1}}^{<}\left(t, t_{1}\right) g_{\mathbf{k} \sigma \eta}^{a}\left(t_{1}, t^{\prime}\right)\right] .
$$

Essa equação é utilizada no cálculo da corrente [veja Eq. 3.14].

É instrutivo, visto que não se encontra na literatura tal discussão, observarmos que a Eq.(A.9) pode ser obtida trocando-se as funções de Green em (A.8) por matrizes [104], definidas da seguinte forma

$$
\begin{aligned}
& \mathbf{G}_{i \mathbf{k} \sigma \eta}=\left(\begin{array}{cc}
G_{i \mathbf{k} \sigma \eta}^{t} & -G_{i \mathbf{k} \sigma \eta}^{<} \\
G_{i \mathbf{k} \sigma \eta}^{>} & -G_{i \mathbf{k} \sigma \eta}^{\overparen{t}}
\end{array}\right), \\
& \mathbf{G}_{i j}=\left(\begin{array}{cc}
G_{i j}^{t} & -G_{i j}^{<} \\
G_{i j}^{>} & -G_{i j}^{\tilde{t}}
\end{array}\right),
\end{aligned}
$$

e

$$
\mathbf{g}_{\mathbf{k} \sigma \eta}=\left(\begin{array}{cc}
g_{\mathbf{k} \sigma \eta}^{t} & -g_{\mathbf{k} \sigma \eta}^{<} \\
g_{\mathbf{k} \sigma \eta}^{>} & -g_{\mathbf{k} \sigma \eta}^{t}
\end{array}\right)
$$

Escrevendo uma equação para essas matrizes análoga a Eq.(A.8) vem

$$
\begin{aligned}
& \left(\begin{array}{cc}
G_{i \mathbf{k} \sigma \eta}^{t}\left(t, t^{\prime}\right) & -G_{i \mathbf{k} \sigma \eta}^{<}\left(t, t^{\prime}\right) \\
G_{i \mathbf{k} \sigma \eta}^{>}\left(t, t^{\prime}\right) & -G_{i \mathbf{k} \sigma \eta}^{\widetilde{t}}\left(t, t^{\prime}\right)
\end{array}\right)=\sum_{i_{1}} t_{\mathbf{k} \sigma}^{*} \frac{(-1)^{i_{1} \delta_{\sigma \downarrow}}}{\sqrt{2}} \int d t_{1} \times \\
& \left(\begin{array}{cc}
G_{i j}^{t}\left(t, t_{1}\right) & -G_{i j}^{<}\left(t, t_{1}\right) \\
G_{i j}^{>}\left(t, t_{1}\right) & -G_{i j}^{\widetilde{t}}\left(t, t_{1}\right)
\end{array}\right)\left(\begin{array}{cc}
g_{\mathbf{k} \sigma \eta}^{t}\left(t_{1}, t^{\prime}\right) & -g_{\mathbf{k} \sigma \eta}^{<}\left(t_{1}, t^{\prime}\right) \\
g_{\mathbf{k} \sigma \eta}^{>}\left(t_{1}, t^{\prime}\right) & -g_{\mathbf{k} \sigma \eta}^{t}\left(t_{1}, t^{\prime}\right)
\end{array}\right) .
\end{aligned}
$$


Tomando a componente matricial $-G_{i \mathbf{k} \sigma \eta}^{<}\left(t, t^{\prime}\right)$ obtemos

$$
G_{i \mathbf{k} \sigma \eta}^{<}\left(t, t^{\prime}\right)=\sum_{i_{1}} t_{\mathbf{k} \sigma}^{*} \frac{(-1)^{i_{1} \delta_{\sigma \downarrow}}}{\sqrt{2}} \int d t_{1}\left[G_{i i_{1}}^{t}\left(t, t_{1}\right) g_{\mathbf{k} \sigma \eta}^{<}\left(t_{1}, t^{\prime}\right)-G_{i i_{1}}^{<}\left(t, t_{1}\right) g_{\mathbf{k} \sigma \eta}^{\tilde{t}}\left(t_{1}, t^{\prime}\right)\right] .
$$

Agora usando as relações $G^{t}=G^{r}+G^{<}$e $g^{\widetilde{t}}=-g^{a}+g^{<}$em (A.14) obtemos (A.9). 


\section{Apêndice B}

\section{Cálculo da expressão para o ruído}

Nesse apêndice apresentamos a dedução da equação do ruído que foi utilizada nos capítulos 3 e 4 [Eq.(3.43)]. O cálculo consiste em se expandir a matriz $S$, que aparece nas funções de Green de quatro operadores [Eqs.(3.38)-(3.41)], e em seguida agrupar termos adequadamente. Vale observar que esse procedimento de expansão da matriz $S$ foi proposto por A. P. Jauho et al. [7] para se calcular corrente, conforme utilizamos no apêndice anterior. Aqui estendemos esse procedimento para o cálculo do ruído.

Comecemos definindo as seguintes funções de Green ordenadas no contorno

$$
\begin{aligned}
g_{1}^{(2)}\left(\tau, \tau^{\prime}\right) & =i^{2}\left\langle T_{c} c_{\mathbf{k} \sigma \eta}^{\dagger}(\tau) d_{i}(\tau) c_{\mathbf{k}^{\prime} \sigma^{\prime} \eta^{\prime}}^{\dagger}\left(\tau^{\prime}\right) d_{j}\left(\tau^{\prime}\right)\right\rangle \\
g_{2}^{(2)}\left(\tau, \tau^{\prime}\right) & =i^{2}\left\langle T_{c} c_{\mathbf{k} \sigma \eta}^{\dagger}(\tau) d_{i}(\tau) d_{j}^{\dagger}\left(\tau^{\prime}\right) c_{\mathbf{k}^{\prime} \sigma^{\prime} \eta^{\prime}}\left(\tau^{\prime}\right)\right\rangle \\
g_{3}^{(2)}\left(\tau, \tau^{\prime}\right) & =i^{2}\left\langle T_{c} d_{i}^{\dagger}(\tau) c_{\mathbf{k} \sigma \eta}(\tau) c_{\mathbf{k}^{\prime} \sigma^{\prime} \eta^{\prime}}^{\dagger}\left(\tau^{\prime}\right) d_{j}\left(\tau^{\prime}\right)\right\rangle \\
g_{4}^{(2)}\left(\tau, \tau^{\prime}\right) & =i^{2}\left\langle T_{c} d_{i}^{\dagger}(\tau) c_{\mathbf{k} \sigma \eta}(\tau) d_{j}^{\dagger}\left(\tau^{\prime}\right) c_{\mathbf{k}^{\prime} \sigma^{\prime} \eta^{\prime}}\left(\tau^{\prime}\right)\right\rangle
\end{aligned}
$$

das quais extraímos $g_{i}^{(2)>}\left(t, t^{\prime}\right)$. Reescrevendo $g_{1}^{(2)}\left(\tau, \tau^{\prime}\right)$ na representação de interação e expandindo a matriz $S=T_{c} e^{-i \int_{C} d \tau_{1} H_{T}\left(\tau_{1}\right)}$, vem

$$
\begin{aligned}
& g_{1}^{(2)}\left(\tau, \tau^{\prime}\right)=i^{2}\left\langle T_{c} S \widetilde{c}_{\mathbf{k} \sigma \eta}^{\dagger}(\tau) \widetilde{d}_{i}(\tau) \widetilde{c}_{\mathbf{k}^{\prime} \sigma^{\prime} \eta^{\prime}}^{\dagger}\left(\tau^{\prime}\right) \widetilde{d}_{j}\left(\tau^{\prime}\right)\right\rangle=i^{2} \sum_{n=2}^{\infty} \frac{(-i)^{n}}{n !} \\
& \left\langle T_{c} \widetilde{c}_{\mathbf{k} \sigma \eta}^{\dagger}(\tau) \widetilde{d}_{i}(\tau) \widetilde{c}_{\mathbf{k}^{\prime} \sigma^{\prime} \eta^{\prime}}^{\dagger}\left(\tau^{\prime}\right) \widetilde{d}_{j}\left(\tau^{\prime}\right) \times\right. \\
& {\left[\int d \tau_{1} \sum_{\mathbf{k}_{1} \sigma_{1} \eta_{1} i_{1}} \frac{(-1)^{i_{1} \delta_{\sigma_{1} \downarrow}}}{\sqrt{2}}\left(t_{\mathbf{k}_{1} \sigma_{1}} \widetilde{c}_{\mathbf{k}_{1} \sigma_{1} \eta_{1}}^{\dagger}\left(\tau_{1}\right) \widetilde{d}_{i_{1}}\left(\tau_{1}\right)+t_{\mathbf{k}_{1} \sigma_{1}}^{*} \widetilde{d}_{i_{1}}^{\dagger}\left(\tau_{1}\right) \widetilde{c}_{\mathbf{k}_{1} \sigma_{1} \eta_{1}}\left(\tau_{1}\right)\right)\right] \times} \\
& {\left[\int d \tau_{2} \sum_{\mathbf{k}_{2} \sigma_{2} \eta_{2} i_{2}} \frac{(-1)^{i_{2} \delta_{\sigma_{2} \downarrow}}}{\sqrt{2}}\left(t_{\mathbf{k}_{2} \sigma_{2}} \widetilde{c}_{\mathbf{k}_{2} \sigma_{2} \eta_{2}}^{\dagger}\left(\tau_{2}\right) \widetilde{d}_{i_{2}}\left(\tau_{2}\right)+t_{\mathbf{k}_{2} \sigma_{2}}^{*} \widetilde{d}_{i_{2}}^{\dagger}\left(\tau_{2}\right) \widetilde{c}_{\mathbf{k}_{2} \sigma_{2} \eta_{2}}\left(\tau_{2}\right)\right)\right] \times} \\
& {\left[\int d \tau_{n} \sum_{\mathbf{k}_{n} \sigma_{n} \eta_{n} i_{n}} \frac{(-1)^{i_{n} \delta_{\sigma_{n} \downarrow} \downarrow}}{\sqrt{2}}\left[t_{\mathbf{k}_{n} \sigma_{n}} \widetilde{c}_{\mathbf{k}_{n} \sigma_{n} \eta_{n}}^{\dagger}\left(\tau_{n}\right) \widetilde{d}_{i_{n}}\left(\tau_{n}\right)+t_{\mathbf{k}_{n} \sigma_{n}}^{*} \widetilde{d}_{i_{n}}^{\dagger}\left(\tau_{n}\right) \widetilde{c}_{\mathbf{k}_{n} \sigma_{n} \eta_{n}}\left(\tau_{n}\right)\right]\right\rangle .}
\end{aligned}
$$


Aplicando o teorema de Wick e reagrupando termos vem

$$
\begin{aligned}
g_{1}^{(2)}\left(\tau, \tau^{\prime}\right)= & i^{2} \sum_{n=2}^{\infty} \frac{(-i)^{n}}{n !} \iint d \tau_{1} d \tau_{2} \sum_{\mathbf{k}_{1} \sigma_{1} \eta_{1} i_{1}} \sum_{\mathbf{k}_{2} \sigma_{2} \eta_{2} i_{2}} t_{\mathbf{k}_{1} \sigma_{1}}^{*} t_{\mathbf{k}_{2} \sigma_{2}}^{*} n(n-1) \times \\
& \frac{(-1)^{i_{1} \delta_{\sigma_{1} \downarrow} \downarrow}}{\sqrt{2}} \frac{(-1)^{i_{2} \delta_{\sigma_{2} \downarrow}}}{\sqrt{2}}(-1)\left\langle T_{c} \widetilde{c}_{\mathbf{k}_{1} \sigma_{1} \eta_{1}}\left(\tau_{1}\right) \widetilde{c}_{\mathbf{k} \sigma \eta}^{\dagger}(\tau)\right\rangle \times \\
& \left\langle T_{c} \widetilde{c}_{\mathbf{k}_{2} \sigma_{2} \eta_{2}}\left(\tau_{2}\right) \widetilde{c}_{\mathbf{k}^{\prime} \sigma^{\prime} \eta^{\prime}}^{\dagger}\left(\tau^{\prime}\right)\right\rangle\left\langle T_{c} \widetilde{d}_{i}(\tau) \widetilde{d}_{j}\left(\tau^{\prime}\right) \widetilde{d}_{i_{1}}^{\dagger}\left(\tau_{1}\right) \widetilde{d}_{i_{2}}^{\dagger}\left(\tau_{2}\right) \int d \tau_{3}\right. \\
& \sum_{\mathbf{k}_{3} \sigma_{3} \eta_{3} i_{3}} \frac{(-1)^{i_{3} \delta_{\sigma_{3} \downarrow} \downarrow}}{\sqrt{2}}\left[t_{\mathbf{k}_{3} \sigma_{3}} \widetilde{c}_{\mathbf{k}_{3} \sigma_{3} \eta_{3}}^{\dagger}\left(\tau_{3}\right) \widetilde{d}_{i_{3}}\left(\tau_{3}\right)+t_{\mathbf{k}_{3} \sigma_{3}}^{*} \widetilde{d}_{i_{3}}^{\dagger}\left(\tau_{3}\right) \widetilde{c}_{\mathbf{k}_{3} \sigma_{3} \eta_{3}}\left(\tau_{3}\right)\right] \times \\
& \cdot \int_{\mathbf{k}_{n} \sigma_{n} \eta_{n} i_{n}} \frac{(-1)^{i_{n} \delta_{\sigma_{n} \downarrow} \downarrow}}{\sqrt{2}}\left[t_{\mathbf{k}_{n} \sigma_{n}} \widetilde{c}_{\mathbf{k}_{n} \sigma_{n} \eta_{n}}^{\dagger}\left(\tau_{n}\right) \widetilde{d}_{i_{n}}\left(\tau_{n}\right)+\right. \\
& \left.\left.\int d \tau_{n} \sum_{\mathbf{k}_{n} \sigma_{n}} \widetilde{d}_{i_{n}}^{\dagger}\left(\tau_{n}\right) \widetilde{c}_{\mathbf{k}_{n} \sigma_{n} \eta_{n}}\left(\tau_{n}\right)\right]\right\rangle .
\end{aligned}
$$

O fator $n(n-1)$ é o número total de contrações possíveis entre os operadores $\widetilde{c}_{\mathbf{k} \sigma \eta}^{\dagger}(\tau)$ e $\widetilde{c}_{\mathbf{k}^{\prime} \sigma^{\prime} \eta^{\prime}}^{\dagger}\left(\tau^{\prime}\right)$ com os operadores $\widetilde{c}_{\mathbf{k}_{i} \sigma_{i} \eta_{i}}^{\dagger}\left(\tau_{i}\right)$ da expansão da matriz $S$. Esse fator cancela parte do fatorial $n$ !, isto é, $n(n-1) / n$ ! $=1 /(n-2)$ !. Com isso, obtemos novamente a expansão da matriz $S$ dentro da terceira média (a médias dos operadores $\widetilde{d}_{i}$ e $\widetilde{d}_{j}^{\dagger}$ ). Isso nos permite reescrever os operadores dessa média novamente nas representações de Heisenberg conforme vemos abaixo.

$$
\begin{gathered}
g_{1}^{(2)}\left(\tau, \tau^{\prime}\right)=i^{2}(-i)^{2} \iint d \tau_{1} d \tau_{2} \sum_{i_{1} i_{2}} t_{\mathbf{k}_{1} \sigma}^{*} t_{\mathbf{k}_{2} \sigma^{\prime}}^{*} \frac{(-1)^{i_{1} \delta_{\sigma \downarrow}}}{\sqrt{2}} \frac{(-1)^{i_{2} \delta_{\sigma^{\prime} \downarrow}}}{\sqrt{2}} \times \\
(-1)\left\langle T_{c} \widetilde{c}_{\mathbf{k} \sigma \eta}\left(\tau_{1}\right) \widetilde{c}_{\mathbf{k} \sigma \eta}^{\dagger}(\tau)\right\rangle\left\langle T_{c} \widetilde{c}_{\mathbf{k}^{\prime} \sigma^{\prime} \eta^{\prime}}\left(\tau_{2}\right) \widetilde{c}_{\mathbf{k}^{\prime} \sigma^{\prime} \eta^{\prime}}^{\dagger}\left(\tau^{\prime}\right)\right\rangle \times \\
\left\langle T_{c} d_{i}(\tau) d_{j}\left(\tau^{\prime}\right) d_{i_{1}}^{\dagger}\left(\tau_{1}\right) d_{i_{2}}^{\dagger}\left(\tau_{2}\right)\right\rangle .
\end{gathered}
$$

Note que as duas primeiras médias (média dos operadores do tipo $\widetilde{c}$ e $\widetilde{c}^{\dagger}$ ) envolve operadores na representação de interação. Portanto, na evolução temporal desses operadores não há acoplamento com o ponto quântico. Definimos essas médias da seguinte forma

$$
g_{\mathbf{k} \sigma \eta}\left(\tau_{1}, \tau\right)=-i\left\langle T_{c} \widetilde{c}_{\mathbf{k}_{1} \sigma_{1} \eta_{1}}\left(\tau_{1}\right) c_{\mathbf{k} \sigma \eta}^{\dagger}(\tau)\right\rangle, \quad g_{\mathbf{k}^{\prime} \sigma^{\prime} \eta^{\prime}}\left(\tau_{2}, \tau^{\prime}\right)=-i\left\langle T_{c} \widetilde{c}_{\mathbf{k}_{2} \sigma_{2} \eta_{2}}\left(\tau_{2}\right) \widetilde{c}_{\mathbf{k}^{\prime} \sigma^{\prime} \eta^{\prime}}^{\dagger}\left(\tau^{\prime}\right)\right\rangle .
$$

Com isso a Eq.(B.3) pode ser reescrita assim

$$
\begin{aligned}
g_{1}^{(2)}\left(\tau, \tau^{\prime}\right)= & -i^{2} \iint d \tau_{1} d \tau_{2} \sum_{i_{1} i_{2}} t_{\mathbf{k}_{1} \sigma}^{*} t_{\mathbf{k}_{2} \sigma^{\prime}}^{*} \frac{(-1)^{i_{1} \delta_{\sigma \downarrow}}}{\sqrt{2}} \frac{(-1)^{i_{2} \delta_{\sigma^{\prime} \downarrow}}}{\sqrt{2}} \times \\
& g_{\mathbf{k} \sigma \eta}\left(\tau_{1}, \tau\right) g_{\mathbf{k}^{\prime} \sigma^{\prime} \eta^{\prime}}\left(\tau_{2}, \tau^{\prime}\right)\left\langle T_{c} d_{i}(\tau) d_{j}\left(\tau^{\prime}\right) d_{i_{1}}^{\dagger}\left(\tau_{1}\right) d_{i_{2}}^{\dagger}\left(\tau_{2}\right)\right\rangle .
\end{aligned}
$$

Como os operadores do tipo $d$ e $d^{\dagger}$ estão na representação de Heisenberg, sua evolução temporal envolve acoplamento com elétrodos, além da interação elétronelétron. Portanto trata-se da parte difícil do problema. Nesse estágio do cálculo efetuamos uma aproximação: quebramos a média de quatro operadores

$$
\left\langle T_{c} d_{i}(\tau) d_{j}\left(\tau^{\prime}\right) d_{i_{1}}^{\dagger}\left(\tau_{1}\right) d_{i_{2}}^{\dagger}\left(\tau_{2}\right)\right\rangle
$$


em produto de médias de dois operadores, da seguinte forma

$$
\begin{aligned}
\left\langle T_{c} d_{i}(\tau) d_{j}\left(\tau^{\prime}\right) d_{i_{1}}^{\dagger}\left(\tau_{1}\right) d_{i_{2}}^{\dagger}\left(\tau_{2}\right)\right\rangle= & \left\langle T_{c} d_{i}(\tau) d_{i_{2}}^{\dagger}\left(\tau_{2}\right)\right\rangle\left\langle T_{c} d_{j}\left(\tau^{\prime}\right) d_{i_{1}}^{\dagger}\left(\tau_{1}\right)\right\rangle \\
& -\left\langle T_{c} d_{i}(\tau) d_{i_{1}}^{\dagger}\left(\tau_{1}\right)\right\rangle\left\langle T_{c} d_{j}\left(\tau^{\prime}\right) d_{i_{2}}^{\dagger}\left(\tau_{2}\right)\right\rangle .
\end{aligned}
$$

Definindo $G_{i j}=-i\left\langle T_{c} d_{i}(\tau) d_{j}\left(\tau^{\prime}\right)\right\rangle$ obtemos para (B.5)

$$
\begin{aligned}
g_{1}^{(2)}\left(\tau, \tau^{\prime}\right)= & \iint d \tau_{1} d \tau_{2} \sum_{i_{1} i_{2}} t_{\mathbf{k} \sigma}^{*} t_{\mathbf{k}^{\prime} \sigma^{\prime}}^{*} \frac{(-1)^{i_{1} \delta_{\sigma \downarrow}}}{\sqrt{2}} \frac{(-1)^{i_{2} \delta_{\sigma^{\prime} \downarrow}}}{\sqrt{2}} \times \\
& g_{\mathbf{k} \sigma \eta}\left(\tau_{1}, \tau\right) g_{\mathbf{k}^{\prime} \sigma^{\prime} \eta^{\prime}}\left(\tau_{2}, \tau^{\prime}\right)\left[G_{i i_{1}}\left(\tau, \tau_{1}\right) G_{j i_{2}}\left(\tau^{\prime}, \tau_{2}\right)-G_{i i_{2}}\left(\tau, \tau_{2}\right) G_{j i_{1}}\left(\tau^{\prime}, \tau_{1}\right)\right] .
\end{aligned}
$$

Expandindo a matriz $S$ em $g_{2}^{(2)}\left(\tau, \tau^{\prime}\right)$ temos

$$
\begin{aligned}
g_{2}^{(2)}\left(\tau, \tau^{\prime}\right)= & i^{2}\left\langle T_{c} S \widetilde{c}_{\mathbf{k} \sigma \eta}^{\dagger}(\tau) \widetilde{d}_{i}(\tau) \widetilde{d}_{j}^{\dagger}\left(\tau^{\prime}\right) \widetilde{c}_{\mathbf{k}^{\prime} \sigma^{\prime} \eta^{\prime}}\left(\tau^{\prime}\right)\right\rangle \\
= & i^{2} \sum_{n=0}^{\infty} \frac{(-i)^{n}}{n !}\left\langleT _ { c } \widetilde { c } _ { \mathbf { k } \sigma \eta } ^ { \dagger } ( \tau ) \widetilde { d } _ { i } ( \tau ) \widetilde { d } _ { j } ^ { \dagger } ( \tau ^ { \prime } ) \widetilde { c } _ { \mathbf { k } ^ { \prime } \sigma ^ { \prime } \eta ^ { \prime } } ( \tau ^ { \prime } ) \left[\int d \tau_{1} \sum_{\mathbf{k}_{1} \sigma_{1} \eta_{1} i_{1}}\right.\right. \\
& \left.\frac{(-1)^{i_{1} \delta_{\sigma_{1} \downarrow}}}{\sqrt{2}}\left(t_{\mathbf{k}_{1} \sigma_{1}} \widetilde{c}_{\mathbf{k}_{1} \sigma_{1} \eta_{1}}^{\dagger}\left(\tau_{1}\right) \widetilde{d}_{i_{1}}\left(\tau_{1}\right)+t_{\mathbf{k}_{1} \sigma_{1}}^{*} \widetilde{d}_{i_{1}}^{\dagger}\left(\tau_{1}\right) \widetilde{c}_{\mathbf{k}_{1} \sigma_{1} \eta_{1}}\left(\tau_{1}\right)\right)\right] \times \\
& \ldots \\
& {\left[\int d \tau_{n} \sum_{\mathbf{k}_{n} \sigma_{n} \eta_{n} i_{n}} \frac{(-1)^{i_{n} \delta_{\sigma_{n} \downarrow}}}{\sqrt{2}} \times\right.} \\
& \left.\left.\left(t_{\mathbf{k}_{n} \sigma_{n}} \widetilde{c}_{\mathbf{k}_{n} \sigma_{n} \eta_{n}}^{\dagger}\left(\tau_{n}\right) \widetilde{d}_{i_{n}}\left(\tau_{n}\right)+t_{\mathbf{k}_{n} \sigma_{n}}^{*} \widetilde{d}_{i_{n}}^{\dagger}\left(\tau_{n}\right) \widetilde{c}_{\mathbf{k}_{n} \sigma_{n} \eta_{n}}\left(\tau_{n}\right)\right)\right]\right\rangle .
\end{aligned}
$$

Agrupando alguns operadores criação e destruição via teorema de Wick obtemos

$$
\begin{aligned}
& g_{2}^{(2)}\left(\tau, \tau^{\prime}\right)=-\delta_{\mathbf{k k}^{\prime}} \delta_{\sigma \sigma^{\prime}} \delta_{\eta \eta^{\prime}} g_{\mathbf{k} \sigma \eta}\left(\tau^{\prime}, \tau\right) G_{i j}\left(\tau, \tau^{\prime}\right)+ \\
& i^{2} \sum_{n=2}^{\infty} \frac{(-i)^{n}}{n !} \iint d \tau_{1} d \tau_{2} \sum_{\mathbf{k}_{1} \sigma_{1} \eta_{1} i_{1}} \sum_{\mathbf{k}_{2} \sigma_{2} \eta_{2} i_{2}} t_{\mathbf{k}_{1} \sigma_{1}}^{*} t_{\mathbf{k}_{2} \sigma_{2}} n(n-1) \\
& \frac{(-1)^{i_{1} \delta_{\sigma_{1} \downarrow}}}{\sqrt{2}} \frac{(-1)^{i_{2} \delta_{\sigma_{2} \downarrow}}}{\sqrt{2}}\left\langle T_{c} \widetilde{c}_{\mathbf{k}_{1} \sigma_{1} \eta_{1}}\left(\tau_{1}\right) \widetilde{c}_{\mathbf{k} \sigma \eta}^{\dagger}(\tau)\right\rangle \times \\
& \left\langle T_{c} \widetilde{c}_{\mathbf{k}^{\prime} \sigma^{\prime} \eta^{\prime}}\left(\tau^{\prime}\right) \widetilde{c}_{\mathbf{k}_{2} \sigma_{2} \eta_{2}}^{\dagger}\left(\tau_{2}\right)\right\rangle\left\langle T_{c} \widetilde{d}_{i}(\tau) \widetilde{d}_{j}^{\dagger}\left(\tau^{\prime}\right) \widetilde{d}_{i_{1}}^{\dagger}\left(\tau_{1}\right) \widetilde{d}_{i_{2}}\left(\tau_{2}\right) \times\right. \\
& \int d \tau_{3} \sum_{\mathbf{k}_{3} \sigma_{3} \eta_{3} i_{3}} \frac{(-1)^{i_{3} \delta_{\sigma_{3} \downarrow}}}{\sqrt{2}} \times \\
& {\left[t_{\mathbf{k}_{3} \sigma_{3}} \widetilde{c}_{\mathbf{k}_{3} \sigma_{3} \eta_{3}}^{\dagger}\left(\tau_{3}\right) \widetilde{d}_{i_{3}}\left(\tau_{3}\right)+t_{\mathbf{k}_{3} \sigma_{3}}^{*} \widetilde{d}_{i_{3}}^{\dagger}\left(\tau_{3}\right) \widetilde{c}_{\mathbf{k}_{3} \sigma_{3} \eta_{3}}\left(\tau_{3}\right)\right] \times} \\
& \int d \tau_{n} \sum_{\mathbf{k}_{n} \sigma_{n} \eta_{n} i_{n}} \frac{(-1)^{i_{n} \delta_{\sigma_{n} \downarrow}}}{\sqrt{2}}\left[t_{\mathbf{k}_{n} \sigma_{n}} \widetilde{c}_{\mathbf{k}_{n} \sigma_{n} \eta_{n}}^{\dagger}\left(\tau_{n}\right) \widetilde{d}_{i_{n}}\left(\tau_{n}\right)+\right. \\
& \left.\left.t_{\mathbf{k}_{n} \sigma_{n}}^{*} \widetilde{d}_{i_{n}}^{\dagger}\left(\tau_{n}\right) \widetilde{c}_{\mathbf{k}_{n} \sigma_{n} \eta_{n}}\left(\tau_{n}\right)\right]\right\rangle,
\end{aligned}
$$

que pode ser reescrita da seguinte forma

$$
\begin{aligned}
g_{2}^{(2)}\left(\tau, \tau^{\prime}\right)= & -\delta_{\mathbf{k k}^{\prime}} \delta_{\sigma \sigma^{\prime}} \delta_{\eta \eta^{\prime}} g_{\mathbf{k} \sigma \eta}\left(\tau^{\prime}, \tau\right) G_{i j}\left(\tau, \tau^{\prime}\right)+ \\
& (-i)^{2} \iint d \tau_{1} d \tau_{2} \sum_{i_{1} i_{2}} t_{\mathbf{k} \sigma}^{*} t_{\mathbf{k}^{\prime} \sigma^{\prime}} \frac{(-1)^{i_{1} \delta_{\sigma \downarrow}}}{\sqrt{2}} \frac{(-1)^{i_{2} \delta_{\sigma^{\prime} \downarrow}}}{\sqrt{2}} \\
& g_{\mathbf{k} \sigma \eta}\left(\tau_{1}, \tau\right) g_{\mathbf{k}^{\prime} \sigma^{\prime} \eta^{\prime}}\left(\tau^{\prime}, \tau_{2}\right)\left\langle T_{c} d_{i}(\tau) d_{j}^{\dagger}\left(\tau^{\prime}\right) d_{i_{1}}^{\dagger}\left(\tau_{1}\right) d_{i_{2}}\left(\tau_{2}\right)\right\rangle .
\end{aligned}
$$


Aproximando

$$
\begin{aligned}
\left\langle T_{c} d_{i}(\tau) d_{j}^{\dagger}\left(\tau^{\prime}\right) d_{i_{1}}^{\dagger}\left(\tau_{1}\right) d_{i_{2}}\left(\tau_{2}\right)\right\rangle= & \left\langle T_{c} d_{i}(\tau) d_{i_{1}}^{\dagger}\left(\tau_{1}\right)\right\rangle\left\langle T_{c} d_{i_{2}}\left(\tau_{2}\right) d_{j}^{\dagger}\left(\tau^{\prime}\right)\right\rangle \\
& -\left\langle T_{c} d_{i}(\tau) d_{j}^{\dagger}\left(\tau^{\prime}\right)\right\rangle\left\langle T_{c} d_{i_{2}}\left(\tau_{2}\right) d_{i_{1}}^{\dagger}\left(\tau_{1}\right)\right\rangle,
\end{aligned}
$$

obtemos

$$
\begin{aligned}
g_{2}^{(2)}\left(\tau, \tau^{\prime}\right)= & -\delta_{\mathbf{k k}^{\prime}} \delta_{\sigma \sigma^{\prime}} \delta_{\eta \eta^{\prime}} g_{\mathbf{k} \sigma \eta}\left(\tau^{\prime}, \tau\right) G_{i j}\left(\tau, \tau^{\prime}\right)+ \\
& \iint d \tau_{1} d \tau_{2} \sum_{i_{1} i_{2}} t_{\mathbf{k} \sigma}^{*} t_{\mathbf{k}^{\prime} \sigma^{\prime}} \frac{(-1)^{i_{1} \delta_{\sigma \downarrow} \downarrow}}{\sqrt{2}} \frac{(-1)^{i_{2} \delta_{\sigma^{\prime} \downarrow}}}{\sqrt{2}} \\
& g_{\mathbf{k} \sigma \eta}\left(\tau_{1}, \tau\right) g_{\mathbf{k}^{\prime} \sigma^{\prime} \eta^{\prime}}\left(\tau^{\prime}, \tau_{2}\right)\left[G_{i i_{1}}\left(\tau, \tau_{1}\right) G_{i_{2} j}\left(\tau_{2}, \tau^{\prime}\right)-G_{i j}\left(\tau, \tau^{\prime}\right) G_{i_{2} i_{1}}\left(\tau_{2}, \tau_{1}\right)\right] .
\end{aligned}
$$

Como $g_{3}^{(2)}\left(\tau, \tau^{\prime}\right)=g_{2}^{(2)^{*}}\left(\tau, \tau^{\prime}\right)$, temos que

$$
\begin{aligned}
g_{3}^{(2)}\left(\tau, \tau^{\prime}\right)= & -\delta_{\mathbf{k k}^{\prime}} \delta_{\sigma \sigma^{\prime}} \delta_{\eta \eta^{\prime}} g_{\mathbf{k} \sigma \eta}\left(\tau, \tau^{\prime}\right) G_{j i}\left(\tau^{\prime}, \tau\right)+ \\
& \iint d \tau_{1} d \tau_{2} \sum_{i_{1} i_{2}} t_{\mathbf{k} \sigma} t_{\mathbf{k}^{\prime} \sigma^{\prime}}^{*} \frac{(-1)^{i_{1} \delta_{\sigma \downarrow} \downarrow}}{\sqrt{2}} \frac{(-1)^{i_{2} \delta_{\sigma^{\prime} \downarrow}}}{\sqrt{2}} \\
& g_{\mathbf{k} \sigma \eta}\left(\tau, \tau_{1}\right) g_{\mathbf{k}^{\prime} \sigma^{\prime} \eta^{\prime}}\left(\tau_{2}, \tau^{\prime}\right)\left[G_{i_{1} i}\left(\tau_{1}, \tau\right) G_{j i_{2}}\left(\tau^{\prime}, \tau_{2}\right)-G_{j i}\left(\tau^{\prime}, \tau\right) G_{i_{1} i_{2}}\left(\tau_{1}, \tau_{2}\right)\right] .
\end{aligned}
$$

Do mesmo modo sendo $g_{4}^{(2)}\left(\tau, \tau^{\prime}\right)=g_{1}^{(2)^{*}}\left(\tau, \tau^{\prime}\right)$ obtemos

$$
\begin{aligned}
g_{4}^{(2)}\left(\tau, \tau^{\prime}\right)= & \iint d \tau_{1} d \tau_{2} \sum_{i_{1} i_{2}} t_{\mathbf{k} \sigma} t_{\mathbf{k}^{\prime} \sigma^{\prime}} \frac{(-1)^{i_{1} \delta_{\sigma \downarrow}}}{\sqrt{2}} \frac{(-1)^{i_{2} \delta_{\sigma^{\prime} \downarrow}}}{\sqrt{2}} \times \\
& g_{\mathbf{k} \sigma \eta}\left(\tau, \tau_{1}\right) g_{\mathbf{k}^{\prime} \sigma^{\prime} \eta^{\prime}}\left(\tau^{\prime}, \tau_{2}\right)\left[G_{i_{1} i}\left(\tau_{1}, \tau\right) G_{i_{2} j}\left(\tau_{2}, \tau^{\prime}\right)-G_{i_{2} i}\left(\tau_{2}, \tau\right) G_{i_{1} j}\left(\tau_{1}, \tau^{\prime}\right)\right] .
\end{aligned}
$$

Tratemos agora apenas o primeiro termo de $g_{1}^{(2)}\left(\tau, \tau^{\prime}\right)$, isto é,

$$
\begin{gathered}
g_{1 \text { desc }}^{(2)}\left(\tau, \tau^{\prime}\right)=t_{\mathbf{k} \sigma}^{*} t_{\mathbf{k}^{\prime} \sigma^{\prime}}^{*} \sum_{i_{1}, i_{2}=1,2} \frac{1}{2}(-1)^{i_{1} \delta_{\sigma \downarrow}}(-1)^{i_{2} \delta_{\sigma^{\prime} \downarrow}} \\
\times \iint d \tau_{1} d \tau_{2} G_{i i_{1}}\left(\tau, \tau_{1}\right) g_{\mathbf{k} \sigma \eta}\left(\tau_{1}, \tau\right) G_{j i_{2}}\left(\tau^{\prime}, \tau_{2}\right) g_{\mathbf{k}^{\prime} \sigma^{\prime} \eta^{\prime}}\left(\tau_{2}, \tau^{\prime}\right) .
\end{gathered}
$$

Esse termo denominamos desconectado pois, se o representarmos através de um diagrama de Feynman, veremos que corresponde a dois diagramas isolados um do outro (diagramas desconectados). Aplicando as regras de Langreth em (B.11) obtemos $g_{1 \text { desc }}^{(2)>}\left(t, t^{\prime}\right)$

$$
\begin{aligned}
& g_{1 \text { desc }}^{(2)>}\left(t, t^{\prime}\right)= t_{\mathbf{k} \sigma}^{*} \sum_{i_{1}} \frac{(-1)^{i_{1} \delta_{\sigma \downarrow}}}{\sqrt{2}} \int d t_{1}\left[G_{i i_{1}}^{r}\left(t, t_{1}\right) g_{\mathbf{k} \sigma \eta}^{<}\left(t_{1}, t\right)+G_{i i_{1}}^{<}\left(t, t_{1}\right) g_{\mathbf{k} \sigma \eta}^{a}\left(t_{1}, t\right)\right] \times \\
& t_{\mathbf{k}^{\prime} \sigma^{\prime}}^{*} \sum_{i_{2}} \frac{(-1)^{i_{2} \delta_{\sigma^{\prime} \downarrow}}}{\sqrt{2}} \int d \tau_{2}\left[G_{j i_{2}}^{r}\left(t^{\prime}, t_{2}\right) g_{\mathbf{k}^{\prime} \sigma^{\prime} \eta^{\prime}}^{<}\left(t_{2}, t^{\prime}\right)+\right. \\
&\left.G_{j i_{2}}^{<}\left(t^{\prime}, t_{2}\right) g_{\mathbf{k}^{\prime} \sigma^{\prime} \eta^{\prime}}^{a}\left(t_{2}, t^{\prime}\right)\right] . \quad(\mathrm{B} .12)
\end{aligned}
$$

Comparando essa expressão com o resultado obtido para $G_{i \mathbf{k} \sigma \eta}^{<}\left(t, t^{\prime}\right)$ concluímos que

$$
g_{1 \operatorname{desc}}^{(2)>}\left(t, t^{\prime}\right)=G_{i \mathbf{k} \sigma \eta}^{<}(t, t) G_{j \mathbf{k}^{\prime} \sigma^{\prime} \eta^{\prime}}^{<}\left(t^{\prime}, t^{\prime}\right)
$$


Como cada função de Green $G_{i \mathbf{k} \sigma \eta}^{<}(t, t)$ corresponde a um diagrama do tipo bubble, vemos novamente que $g_{1 \text { desc }}^{(2)>}\left(t, t^{\prime}\right)$ equivale a um diagrama de Feynman desconectado. Analisando agora a primeira parte do segundo termo de $g_{2}^{(2)}$, isto é, o termo desconectado

$$
\begin{aligned}
g_{2 d e s c}^{(2)}\left(\tau, \tau^{\prime}\right)= & t_{\mathbf{k} \sigma}^{*} t_{\mathbf{k}^{\prime} \sigma^{\prime}} \sum_{i_{1} i_{2}} \frac{(-1)^{i_{1} \delta_{\sigma \downarrow}}}{\sqrt{2}} \frac{(-1)^{i_{2} \delta_{\sigma^{\prime} \downarrow}}}{\sqrt{2}} \\
& \iint d \tau_{1} d \tau_{2} G_{i i_{1}}\left(\tau, \tau_{1}\right) g_{\mathbf{k} \sigma \eta}\left(\tau_{1}, \tau\right) g_{\mathbf{k}^{\prime} \sigma^{\prime} \eta^{\prime}}\left(\tau^{\prime}, \tau_{2}\right) G_{i_{2} j}\left(\tau_{2}, \tau^{\prime}\right), \mathrm{B}
\end{aligned}
$$

obtemos

$$
\begin{aligned}
g_{2 d e s c}^{(2)>}\left(t, t^{\prime}\right) & =t_{\mathbf{k} \sigma}^{*} \sum_{i_{1}} \frac{(-1)^{i_{1} \delta_{\sigma \downarrow}}}{\sqrt{2}} \int d t_{1}\left[G_{i i_{1}}^{r}\left(t, t_{1}\right) g_{\mathbf{k} \sigma \eta}^{<}\left(t_{1}, t\right)+G_{i i_{1}}^{<}\left(t, t_{1}\right) g_{\mathbf{k} \sigma \eta}^{a}\left(t_{1}, t\right)\right] \times \\
t_{\mathbf{k}^{\prime} \sigma^{\prime}} & \sum_{i_{2}} \frac{(-1)^{i_{2} \delta_{\sigma^{\prime} \downarrow}}}{\sqrt{2}} \int d \tau_{2}\left[g_{\mathbf{k}^{\prime} \sigma^{\prime} \eta^{\prime}}^{r}\left(t^{\prime}, t_{2}\right) G_{i_{2} j}^{<}\left(t_{2}, t^{\prime}\right)+g_{\mathbf{k}^{\prime} \sigma^{\prime} \eta^{\prime}}^{<}\left(t^{\prime}, t_{2}\right) G_{i_{2} j}^{a}\left(t_{2}, t^{\prime}\right)\right] \\
& =-G_{i \mathbf{k} \sigma \eta}^{<}(t, t) G_{j \mathbf{k}^{\prime} \sigma^{\prime} \eta^{\prime}}^{<*}\left(t^{\prime}, t^{\prime}\right) .
\end{aligned}
$$

Temos também

$$
\begin{aligned}
g_{3 \operatorname{desc}}^{(2)>}\left(t, t^{\prime}\right) & =t_{\mathbf{k} \sigma} \sum_{i_{1}} \frac{(-1)^{i_{1} \delta_{\sigma \downarrow}}}{\sqrt{2}} \int d t_{1}\left[g_{\mathbf{k} \sigma \eta}^{r}\left(t, t_{1}\right) G_{i_{1} i}^{<}\left(t_{1}, t\right)+g_{\mathbf{k} \sigma \eta}^{<}\left(t, t_{1}\right) G_{i_{1} i}^{a}\left(t_{1}, t\right)\right] \times \\
t_{\mathbf{k}^{\prime} \sigma^{\prime}}^{*} & \sum_{i_{2}} \frac{(-1)^{i_{2} \delta_{\sigma^{\prime} \downarrow}}}{\sqrt{2}} \int d \tau_{2}\left[G_{j i_{2}}^{r}\left(t^{\prime}, t_{2}\right) g_{\mathbf{k}^{\prime} \sigma^{\prime} \eta^{\prime}}^{<}\left(t_{2}, t^{\prime}\right)+G_{j i_{2}}^{<}\left(t^{\prime}, t_{2}\right) g_{\mathbf{k}^{\prime} \sigma^{\prime} \eta^{\prime}}^{a}\left(t_{2}, t^{\prime}\right)\right] \\
= & -G_{i \mathbf{k} \sigma \eta}^{<*}(t, t) G_{j \mathbf{k}^{\prime} \sigma^{\prime} \eta^{\prime}}^{<}\left(t^{\prime}, t^{\prime}\right),
\end{aligned}
$$

e

$$
\begin{aligned}
g_{4 d e s c}^{(2)>}\left(t, t^{\prime}\right) & =t_{\mathbf{k} \sigma} \sum_{i_{1}} \frac{(-1)^{i_{1} \delta_{\sigma \downarrow}}}{\sqrt{2}} \int d t_{1}\left[g_{\mathbf{k} \sigma \eta}^{r}\left(t, t_{1}\right) G_{i_{1} i}^{<}\left(t_{1}, t\right)+g_{\mathbf{k} \sigma \eta}^{<}\left(t, t_{1}\right) G_{i_{1} i}^{a}\left(t_{1}, t\right)\right] \times \\
t_{\mathbf{k}^{\prime} \sigma^{\prime}}^{*} & \sum_{i_{2}} \frac{(-1)^{i_{2} \delta_{\sigma^{\prime} \downarrow}}}{\sqrt{2}} \int d \tau_{2}\left[g_{\mathbf{k}^{\prime} \sigma^{\prime} \eta^{\prime}}^{r}\left(t^{\prime}, t_{2}\right) G_{i_{2 j}}^{<}\left(t_{2}, t^{\prime}\right)+g_{\mathbf{k}^{\prime} \sigma^{\prime} \eta^{\prime}}^{<}\left(t^{\prime}, t_{2}\right) G_{i_{2 j} j}^{a}\left(t_{2}, t^{\prime}\right)\right] \\
= & G_{i \mathbf{k} \sigma \eta}^{<*}(t, t) G_{j \mathbf{k}^{\prime} \sigma^{\prime} \eta^{\prime}}^{<^{*}}\left(t^{\prime}, t^{\prime}\right) .
\end{aligned}
$$

Substituindo somente os termos $g_{i d e s c}^{(2)>}\left(t, t^{\prime}\right)$ na expressão do ruído vem

$$
\begin{aligned}
S_{\eta \eta^{\prime}}^{\text {desc }}\left(t, t^{\prime}\right)= & \left(\frac{e}{\hbar}\right)^{2} \sum_{\mathbf{k} \mathbf{k}^{\prime} \sigma \sigma^{\prime} i j} \frac{1}{2}(-1)^{i \delta_{\sigma \downarrow}}(-1)^{j \delta_{\sigma^{\prime} \downarrow}} \\
& \left\{t_{\mathbf{k} \sigma} t_{\mathbf{k}^{\prime} \sigma^{\prime}} G_{i \mathbf{k} \sigma \eta}^{<}(t, t) G_{j \mathbf{k}^{\prime} \sigma^{\prime} \eta^{\prime}}^{<}\left(t^{\prime}, t^{\prime}\right)-t_{\mathbf{k} \sigma} t_{\mathbf{k}^{\prime} \sigma^{\prime}}^{*} G_{i \mathbf{k} \sigma \eta}^{<}(t, t) G_{j \mathbf{k}^{\prime} \sigma^{\prime} \eta^{\prime}}^{<*}\left(t^{\prime}, t^{\prime}\right)\right. \\
& \left.-t_{\mathbf{k} \sigma}^{*} t_{\mathbf{k}^{\prime} \sigma^{\prime}} G_{i \mathbf{k} \sigma \eta}^{<*}(t, t) G_{j \mathbf{k}^{\prime} \sigma^{\prime} \eta^{\prime}}^{<}\left(t^{\prime}, t^{\prime}\right)+t_{\mathbf{k} \sigma}^{*} t_{\mathbf{k}^{\prime} \sigma^{\prime}}^{*} G_{i \mathbf{k} \sigma \eta}^{<*}(t, t) G_{j \mathbf{k}^{\prime} \sigma^{\prime} \eta^{\prime}}^{<*}\left(t^{\prime}, t^{\prime}\right)\right\} \\
& +h . c .-2 I_{\eta}^{2} \\
= & \left.2\left(\frac{e}{\hbar}\right)^{2} \sum_{\mathbf{k} \mathbf{k}^{\prime} \sigma \sigma^{\prime} i j} \frac{1}{2}(-1)^{i \delta_{\sigma \downarrow} \downarrow}(-1)^{j \delta_{\sigma^{\prime} \downarrow}\left[t_{\mathbf{k} \sigma} G_{i \mathbf{k} \sigma \eta}\right.}(t, t)-t_{\mathbf{k} \sigma}^{*} G_{i \mathbf{k} \sigma \eta}^{<*}(t, t)\right] \times \\
& {\left[t_{\mathbf{k}^{\prime} \sigma^{\prime}} G_{j \mathbf{k}^{\prime} \sigma^{\prime} \eta^{\prime}}^{<}\left(t^{\prime}, t^{\prime}\right)-t_{\mathbf{k}^{\prime} \sigma^{\prime}}^{*} G_{j \mathbf{k}^{\prime} \sigma^{\prime} \eta^{\prime}}^{<}\left(t^{\prime}, t^{\prime}\right)\right]-2 I_{\eta}^{2} . \quad \text { (B.18) } }
\end{aligned}
$$


Como

$$
I_{\eta}=\left\langle I_{\eta}(t)\right\rangle=\frac{e}{\hbar} \sum_{\mathbf{k} \sigma i} \frac{(-1)^{i \delta_{\sigma \downarrow}}}{\sqrt{2}}\left[t_{\mathbf{k} \sigma} G_{i \mathbf{k} \sigma \eta}^{<}(t, t)-t_{\mathbf{k} \sigma}^{*} G_{i \mathbf{k} \sigma \eta}^{<^{*}}(t, t)\right],
$$

temos que

$$
\begin{array}{r}
I_{\eta} I_{\eta^{\prime}}=\left\langle I_{\eta}(t)\right\rangle\left\langle I_{\eta}\left(t^{\prime}\right)\right\rangle=\left(\frac{e}{\hbar}\right)^{2} \sum_{\mathbf{k} \mathbf{k}^{\prime} \sigma \sigma^{\prime} i j} \frac{(-1)^{i \delta_{\sigma \downarrow}}}{\sqrt{2}} \frac{(-1)^{j \delta_{\sigma \downarrow}}}{\sqrt{2}}\left[t_{\mathbf{k} \sigma} G_{i \mathbf{k} \sigma \eta}^{<}(t, t)-t_{\mathbf{k} \sigma}^{*} G_{i \mathbf{k} \sigma \eta}^{*}(t, t)\right] \times \\
{\left[t_{\mathbf{k}^{\prime} \sigma^{\prime}} G_{j \mathbf{k}^{\prime} \sigma^{\prime} \eta^{\prime}}^{<}\left(t^{\prime}, t^{\prime}\right)-t_{\mathbf{k}^{\prime} \sigma^{\prime}}^{*} G_{j \mathbf{k}^{\prime} \sigma^{\prime} \eta^{\prime}}^{<*}\left(t^{\prime}, t^{\prime}\right)\right] .}
\end{array}
$$

Substituindo (B.20) em (B.18) vemos que

$$
S_{\eta \eta^{\prime}}^{\text {desc }}\left(t, t^{\prime}\right)=0
$$

Com isso vemos que somente os termos conectados contribuem para o ruído. Desse modo a expressão para o ruído fica

$$
\begin{aligned}
& S_{\eta \eta^{\prime}}\left(t, t^{\prime}\right)=\left\{\left(\frac{e}{\hbar}\right)^{2} \sum_{\mathbf{k} \sigma i j} \frac{1}{2}(-1)^{(i+j) \delta_{\sigma \downarrow}}\left|t_{\mathbf{k} \sigma}\right|^{2} \times\right. \\
& \left.-\left(\frac{e}{\hbar}\right)^{2} \sum_{\mathbf{k} \mathbf{k}^{\prime} \sigma \sigma^{\prime}} \sum_{i j i_{1} i_{2}}\left(\tau, \tau^{\prime}\right) G_{j i}\left(\tau^{\prime}, \tau\right)+g_{\mathbf{k} \sigma \eta}\left(\tau^{\prime}, \tau\right) G_{i j}\left(\tau, \tau^{\prime}\right)\right] \\
& {\left[G_{i i_{2}}\left(\tau, \tau_{2}\right) g_{\mathbf{k}^{\prime} \sigma^{\prime} \eta^{\prime}}\left(\tau_{2}, \tau^{\prime}\right) G_{j i_{1}}\left(\tau^{\prime}, \tau_{1}\right) g_{\mathbf{k} \sigma \eta}\left(\tau_{1}, \tau\right)-\right.} \\
& G_{i j}\left(\tau, \tau^{\prime}\right) g_{\mathbf{k}^{\prime} \sigma^{\prime} \eta^{\prime}}\left(\tau^{\prime}, \tau_{2}\right) G_{i_{2} i_{1}}\left(\tau_{2}, \tau_{1}\right) g_{\mathbf{k} \sigma \eta}\left(\tau_{1}, \tau\right)+ \\
& g_{\mathbf{k} \sigma \eta}\left(\tau, \tau_{1}\right) G_{i_{1} j}\left(\tau_{1}, \tau^{\prime}\right) g_{\mathbf{k}^{\prime} \sigma^{\prime} \eta^{\prime}}\left(\tau^{\prime}, \tau_{2}\right) G_{i_{2} i}\left(\tau_{2}, \tau\right)- \\
& g_{\mathbf{k} \sigma \eta}\left(\tau,\left.\tau_{\mathbf{k}^{\prime} \sigma^{\prime}}\right|^{2} \int d \tau_{1} d \tau_{2} \times\right. \\
& \left.\left.G_{i_{1} i_{2}}\left(\tau_{1}, \tau_{2}\right) g_{\mathbf{k}^{\prime} \sigma^{\prime} \eta^{\prime}}\left(\tau_{2}, \tau^{\prime}\right) G_{j i}\left(\tau^{\prime}, \tau\right)\right]\right\}^{t>t^{\prime}}+c . c . .
\end{aligned}
$$

Onde o super-escrito $t>t^{\prime}$ significa que a continuação analítica com esse ordena- 
mento deve ser aplicada. Aplicando as regras de Langreth obtemos

$$
\begin{aligned}
& S_{\eta \eta^{\prime}}\left(t, t^{\prime}\right)=\left(\frac{e}{\hbar}\right)^{2} \sum_{\mathbf{k} \sigma i j} \frac{1}{2}(-1)^{(i+j) \delta_{\sigma \downarrow}}\left|t_{\mathbf{k} \sigma}\right|^{2}\left[g_{\mathbf{k} \sigma \eta}^{>}\left(t, t^{\prime}\right) G_{j i}^{<}\left(t^{\prime}, t\right)+g_{\mathbf{k} \sigma \eta}^{<}\left(t^{\prime}, t\right) G_{i j}^{>}\left(t, t^{\prime}\right)\right] \\
& -\left(\frac{e}{\hbar}\right)^{2} \sum_{\mathbf{k k}^{\prime} \sigma \sigma^{\prime}} \sum_{i j i_{1} i_{2}} \frac{1}{4}(-1)^{\left(i+i_{1}\right) \delta_{\sigma \downarrow}}(-1)^{\left(j+i_{2}\right) \delta_{\sigma^{\prime}}}\left|t_{\mathbf{k} \sigma}\right|^{2}\left|t_{\mathbf{k}^{\prime} \sigma^{\prime}}\right|^{2} \iint d t_{1} d t_{2} \times \\
& {\left[G_{i i_{2}}^{>}\left(t, t_{2}\right) g_{\mathbf{k}^{\prime} \sigma^{\prime} \eta^{\prime}}^{a}\left(t_{2}, t^{\prime}\right) G_{j i_{1}}^{r}\left(t^{\prime}, t_{1}\right) g_{\mathbf{k} \sigma \eta}^{<}\left(t_{1}, t\right)\right.} \\
& +G_{i i_{2}}^{r}\left(t, t_{2}\right) g_{\mathbf{k}^{\prime} \sigma^{\prime} \eta^{\prime}}^{>}\left(t_{2}, t^{\prime}\right) G_{j i_{1}}^{r}\left(t^{\prime}, t_{1}\right) g_{\mathbf{k} \sigma \eta}^{<}\left(t_{1}, t\right) \\
& +G_{i i_{2}}^{>}\left(t, t_{2}\right) g_{\mathbf{k}^{\prime} \sigma^{\prime} \eta^{\prime}}^{a}\left(t_{2}, t^{\prime}\right) G_{j i_{1}}^{<}\left(t^{\prime}, t_{1}\right) g_{\mathbf{k} \sigma \eta}^{a}\left(t_{1}, t\right) \\
& +G_{i i_{2}}^{r}\left(t, t_{2}\right) g_{\mathbf{k}^{\prime} \sigma^{\prime} \eta^{\prime}}^{>}\left(t_{2}, t^{\prime}\right) G_{j i_{1}}^{<}\left(t^{\prime}, t_{1}\right) g_{\mathbf{k} \sigma \eta}^{a}\left(t_{1}, t\right) \\
& \text { - } G_{i j}^{>}\left(t, t^{\prime}\right) g_{\mathbf{k}^{\prime} \sigma^{\prime} \eta^{\prime}}^{r}\left(t^{\prime}, t_{2}\right) G_{i_{2} i_{1}}^{r}\left(t_{2}, t_{1}\right) g_{\mathbf{k} \sigma \eta}^{<}\left(t_{1}, t\right) \\
& \text { - } G_{i j}^{>}\left(t, t^{\prime}\right) g_{\mathbf{k}^{\prime} \sigma^{\prime} \eta^{\prime}}^{r}\left(t^{\prime}, t_{2}\right) G_{i_{2} i_{1}}^{<}\left(t_{2}, t_{1}\right) g_{\mathbf{k} \sigma \eta}^{a}\left(t_{1}, t\right) \\
& \text { - } G_{i j}^{>}\left(t, t^{\prime}\right) g_{\mathbf{k}^{\prime} \sigma^{\prime} \eta^{\prime}}^{<}\left(t^{\prime}, t_{2}\right) G_{i_{2} i_{1}}^{a}\left(t_{2}, t_{1}\right) g_{\mathbf{k} \sigma \eta}^{a}\left(t_{1}, t\right) \\
& \text { - } g_{\mathbf{k} \sigma \eta}^{>}\left(t, t_{1}\right) G_{i_{1} i_{2}}^{a}\left(t_{1}, t_{2}\right) g_{\mathbf{k}^{\prime} \sigma^{\prime} \eta^{\prime}}^{a}\left(t_{2}, t^{\prime}\right) G_{j i}^{<}\left(t^{\prime}, t\right) \\
& \text { - } g_{\mathbf{k} \sigma \eta}^{r}\left(t, t_{1}\right) G_{i_{1} i_{2}}^{>}\left(t_{1}, t_{2}\right) g_{\mathbf{k}^{\prime} \sigma^{\prime} \eta^{\prime}}^{a}\left(t_{2}, t^{\prime}\right) G_{j i}^{<}\left(t^{\prime}, t\right) \\
& \text { - } g_{\mathbf{k} \sigma \eta}^{r}\left(t, t_{1}\right) G_{i_{1} i_{2}}^{r}\left(t_{1}, t_{2}\right) g_{\mathbf{k}^{\prime} \sigma^{\prime} \eta^{\prime}}^{>}\left(t_{2}, t^{\prime}\right) G_{j i}^{<}\left(t^{\prime}, t\right) \\
& +g_{\mathbf{k} \sigma \eta}^{>}\left(t, t_{1}\right) G_{i_{1 j} j}^{a}\left(t_{1}, t^{\prime}\right) g_{\mathbf{k}^{\prime} \sigma^{\prime} \eta^{\prime}}^{r}\left(t^{\prime}, t_{2}\right) G_{i_{2} i}^{<}\left(t_{2}, t\right) \\
& +g_{\mathbf{k} \sigma \eta}^{>}\left(t, t_{1}\right) G_{i_{1} j}^{a}\left(t_{1}, t^{\prime}\right) g_{\mathbf{k}^{\prime} \sigma^{\prime} \eta^{\prime}}^{<}\left(t^{\prime}, t_{2}\right) G_{i_{2} i}^{a}\left(t_{2}, t\right) \\
& +g_{\mathbf{k} \sigma \eta}^{r}\left(t, t_{1}\right) G_{i_{1} j}^{>}\left(t_{1}, t^{\prime}\right) g_{\mathbf{k}^{\prime} \sigma^{\prime} \eta^{\prime}}^{r}\left(t^{\prime}, t_{2}\right) G_{i_{2} i}^{<}\left(t_{2}, t\right) \\
& \left.+g_{\mathbf{k} \sigma \eta}^{r}\left(t, t_{1}\right) G_{i_{1} j}^{>}\left(t_{1}, t^{\prime}\right) g_{\mathbf{k}^{\prime} \sigma^{\prime} \eta^{\prime}}^{<}\left(t^{\prime}, t_{2}\right) G_{i_{2} i}^{a}\left(t_{2}, t\right)\right]+ \text { c.c }
\end{aligned}
$$


Escrevendo explicitamente o termo complexo conjugado (c.c.) vem

$$
\begin{aligned}
& S_{\eta \eta^{\prime}}\left(t, t^{\prime}\right)=\left(\frac{e}{\hbar}\right)^{2} \sum_{\mathbf{k} \sigma i j} \frac{1}{2}(-1)^{(i+j) \delta_{\sigma \downarrow}}\left|t_{\mathbf{k} \sigma}\right|^{2}\left[g_{\mathbf{k} \sigma \eta}^{>}\left(t, t^{\prime}\right) G_{j i}^{<}\left(t^{\prime}, t\right)+g_{\mathbf{k} \sigma \eta}^{<}\left(t^{\prime}, t\right) G_{i j}^{>}\left(t, t^{\prime}\right)+\right. \\
& \left.g_{\mathbf{k} \sigma \eta}^{<}\left(t, t^{\prime}\right) G_{j i}^{>}\left(t^{\prime}, t\right)+g_{\mathbf{k} \sigma \eta}^{>}\left(t^{\prime}, t\right) G_{i j}^{<}\left(t, t^{\prime}\right)\right]-
\end{aligned}
$$

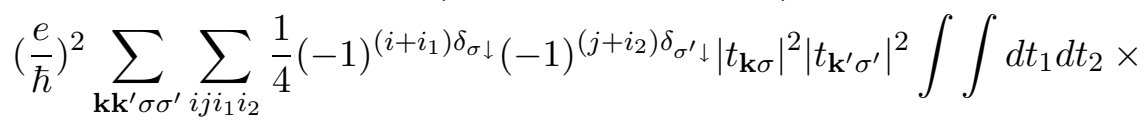

$$
\begin{aligned}
& {\left[G_{i i_{2}}^{>}\left(t, t_{2}\right) g_{\mathbf{k}^{\prime} \sigma^{\prime} \eta^{\prime}}^{a}\left(t_{2}, t^{\prime}\right) G_{j i_{1}}^{r}\left(t^{\prime}, t_{1}\right) g_{\mathbf{k} \sigma \eta}^{<}\left(t_{1}, t\right)\right.} \\
& +G_{i i_{2}}^{r}\left(t, t_{2}\right) g_{\mathbf{k}^{\prime} \sigma^{\prime} \eta^{\prime}}^{>}\left(t_{2}, t^{\prime}\right) G_{j i_{1}}^{r}\left(t^{\prime}, t_{1}\right) g_{\mathbf{k} \sigma \eta}^{<}\left(t_{1}, t\right) \\
& +G_{i i_{2}}^{>}\left(t, t_{2}\right) g_{\mathbf{k}^{\prime} \sigma^{\prime} \eta^{\prime}}^{a}\left(t_{2}, t^{\prime}\right) G_{j i_{1}}^{<}\left(t^{\prime}, t_{1}\right) g_{\mathbf{k} \sigma \eta}^{a}\left(t_{1}, t\right) \\
& +G_{i i_{2}}^{r}\left(t, t_{2}\right) g_{\mathbf{k}^{\prime} \sigma^{\prime} \eta^{\prime}}^{>}\left(t_{2}, t^{\prime}\right) G_{j i_{1}}^{<}\left(t^{\prime}, t_{1}\right) g_{\mathbf{k} \sigma \eta}^{a}\left(t_{1}, t\right) \\
& \text { - } G_{i j}^{>}\left(t, t^{\prime}\right) g_{\mathbf{k}^{\prime} \sigma^{\prime} \eta^{\prime}}^{r}\left(t^{\prime}, t_{2}\right) G_{i_{2} i_{1}}^{r}\left(t_{2}, t_{1}\right) g_{\mathbf{k} \sigma \eta}^{<}\left(t_{1}, t\right) \\
& \text { - } G_{i j}^{>}\left(t, t^{\prime}\right) g_{\mathbf{k}^{\prime} \sigma^{\prime} \eta^{\prime}}^{r}\left(t^{\prime}, t_{2}\right) G_{i_{2} i_{1}}^{<}\left(t_{2}, t_{1}\right) g_{\mathbf{k} \sigma \eta}^{a}\left(t_{1}, t\right) \\
& \text { - } G_{i j}^{>}\left(t, t^{\prime}\right) g_{\mathbf{k}^{\prime} \sigma^{\prime} \eta^{\prime}}^{<}\left(t^{\prime}, t_{2}\right) G_{i_{2} i_{1}}^{a}\left(t_{2}, t_{1}\right) g_{\mathbf{k} \sigma \eta}^{a}\left(t_{1}, t\right) \\
& \text { - } g_{\mathbf{k} \sigma \eta}^{>}\left(t, t_{1}\right) G_{i_{1} i_{2}}^{a}\left(t_{1}, t_{2}\right) g_{\mathbf{k}^{\prime} \sigma^{\prime} \eta^{\prime}}^{a}\left(t_{2}, t^{\prime}\right) G_{j i}^{<}\left(t^{\prime}, t\right) \\
& \text { - } g_{\mathbf{k} \sigma \eta}^{r}\left(t, t_{1}\right) G_{i_{1} i_{2}}^{>}\left(t_{1}, t_{2}\right) g_{\mathbf{k}^{\prime} \sigma^{\prime} \eta^{\prime}}^{a}\left(t_{2}, t^{\prime}\right) G_{j i}^{<}\left(t^{\prime}, t\right) \\
& \text { - } g_{\mathbf{k} \sigma \eta}^{r}\left(t, t_{1}\right) G_{i_{1} i_{2}}^{r}\left(t_{1}, t_{2}\right) g_{\mathbf{k}^{\prime} \sigma^{\prime} \eta^{\prime}}^{>}\left(t_{2}, t^{\prime}\right) G_{j i}^{<}\left(t^{\prime}, t\right) \\
& +g_{\mathbf{k} \sigma \eta}^{>}\left(t, t_{1}\right) G_{i_{1} j}^{a}\left(t_{1}, t^{\prime}\right) g_{\mathbf{k}^{\prime} \sigma^{\prime} \eta^{\prime}}^{r}\left(t^{\prime}, t_{2}\right) G_{i_{2} i}^{<}\left(t_{2}, t\right) \\
& +g_{\mathbf{k} \sigma \eta}^{>}\left(t, t_{1}\right) G_{i_{1} j}^{a}\left(t_{1}, t^{\prime}\right) g_{\mathbf{k}^{\prime} \sigma^{\prime} \eta^{\prime}}^{<}\left(t^{\prime}, t_{2}\right) G_{i_{2} i}^{a}\left(t_{2}, t\right) \\
& +g_{\mathbf{k} \sigma \eta}^{r}\left(t, t_{1}\right) G_{i_{1} j}^{>}\left(t_{1}, t^{\prime}\right) g_{\mathbf{k}^{\prime} \sigma^{\prime} \eta^{\prime}}^{r}\left(t^{\prime}, t_{2}\right) G_{i_{2} i}^{<}\left(t_{2}, t\right) \\
& +g_{\mathbf{k} \sigma \eta}^{r}\left(t, t_{1}\right) G_{i_{1} j}^{>}\left(t_{1}, t^{\prime}\right) g_{\mathbf{k}^{\prime} \sigma^{\prime} \eta^{\prime}}^{<}\left(t^{\prime}, t_{2}\right) G_{i_{2} i}^{a}\left(t_{2}, t\right) \\
& +g_{\mathbf{k} \sigma \eta}^{<}\left(t, t_{1}\right) G_{i_{1} j}^{a}\left(t_{1}, t^{\prime}\right) g_{\mathbf{k}^{\prime} \sigma^{\prime} \eta^{\prime}}^{r}\left(t^{\prime}, t_{2}\right) G_{i_{2} i}^{>}\left(t_{2}, t\right) \\
& +g_{\mathbf{k} \sigma \eta}^{<}\left(t, t_{1}\right) G_{i_{1} j}^{a}\left(t_{1}, t^{\prime}\right) g_{\mathbf{k}^{\prime} \sigma^{\prime} \eta^{\prime}}^{>}\left(t^{\prime}, t_{2}\right) G_{i_{2} i}^{a}\left(t_{2}, t\right) \\
& +g_{\mathbf{k} \sigma \eta}^{r}\left(t, t_{1}\right) G_{i_{1} j}^{<}\left(t_{1}, t^{\prime}\right) g_{\mathbf{k}^{\prime} \sigma^{\prime} \eta^{\prime}}^{r}\left(t^{\prime}, t_{2}\right) G_{i_{2} i}^{>}\left(t_{2}, t\right) \\
& +g_{\mathbf{k} \sigma \eta}^{r}\left(t, t_{1}\right) G_{i_{1} j}^{<}\left(t_{1}, t^{\prime}\right) g_{\mathbf{k}^{\prime} \sigma^{\prime} \eta^{\prime}}^{>}\left(t^{\prime}, t_{2}\right) G_{i_{2} i}^{a}\left(t_{2}, t\right) \\
& \text { - } g_{\mathbf{k} \sigma \eta}^{<}\left(t, t_{1}\right) G_{i_{1} i_{2}}^{a}\left(t_{1}, t_{2}\right) g_{\mathbf{k}^{\prime} \sigma^{\prime} \eta^{\prime}}^{a}\left(t_{2}, t^{\prime}\right) G_{j i}^{>}\left(t^{\prime}, t\right) \\
& \text { - } g_{\mathbf{k} \sigma \eta}^{r}\left(t, t_{1}\right) G_{i_{1} i_{2}}^{<}\left(t_{1}, t_{2}\right) g_{\mathbf{k}^{\prime} \sigma^{\prime} \eta^{\prime}}^{a}\left(t_{2}, t^{\prime}\right) G_{j i}^{>}\left(t^{\prime}, t\right) \\
& \text { - } g_{\mathbf{k} \sigma \eta}^{r}\left(t, t_{1}\right) G_{i_{1} i_{2}}^{r}\left(t_{1}, t_{2}\right) g_{\mathbf{k}^{\prime} \sigma^{\prime} \eta^{\prime}}^{<}\left(t_{2}, t^{\prime}\right) G_{j i}^{>}\left(t^{\prime}, t\right) \\
& \text { - } G_{i j}^{<}\left(t, t^{\prime}\right) g_{\mathbf{k}^{\prime} \sigma^{\prime} \eta^{\prime}}^{r}\left(t^{\prime}, t_{2}\right) G_{i_{2} i_{1}}^{r}\left(t_{2}, t_{1}\right) g_{\mathbf{k} \sigma \eta}^{>}\left(t_{1}, t\right) \\
& \text { - } G_{i j}^{<}\left(t, t^{\prime}\right) g_{\mathbf{k}^{\prime} \sigma^{\prime} \eta^{\prime}}^{r}\left(t^{\prime}, t_{2}\right) G_{i_{2} i_{1}}^{>}\left(t_{2}, t_{1}\right) g_{\mathbf{k} \sigma \eta}^{a}\left(t_{1}, t\right) \\
& \text { - } G_{i j}^{<}\left(t, t^{\prime}\right) g_{\mathbf{k}^{\prime} \sigma^{\prime} \eta^{\prime}}^{>}\left(t^{\prime}, t_{2}\right) G_{i_{2} i_{1}}^{a}\left(t_{2}, t_{1}\right) g_{\mathbf{k} \sigma \eta}^{a}\left(t_{1}, t\right) \\
& +G_{i i_{2}}^{r}\left(t, t_{2}\right) g_{\mathbf{k}^{\prime} \sigma^{\prime} \eta^{\prime}}^{<}\left(t_{2}, t^{\prime}\right) G_{j i_{1}}^{r}\left(t^{\prime}, t_{1}\right) g_{\mathbf{k} \sigma \eta}^{>}\left(t_{1}, t\right) \\
& +G_{i i_{2}}^{<}\left(t, t_{2}\right) g_{\mathbf{k}^{\prime} \sigma^{\prime} \eta^{\prime}}^{a}\left(t_{2}, t^{\prime}\right) G_{j i_{1}}^{r}\left(t^{\prime}, t_{1}\right) g_{\mathbf{k} \sigma \eta}^{>}\left(t_{1}, t\right) \\
& +G_{i i_{2}}^{<}\left(t, t_{2}\right) g_{\mathbf{k}^{\prime} \sigma^{\prime} \eta^{\prime}}^{a}\left(t_{2}, t^{\prime}\right) G_{j i_{1}}^{>}\left(t^{\prime}, t_{1}\right) g_{\mathbf{k} \sigma \eta}^{a}\left(t_{1}, t\right) \\
& \left.+G_{i i_{2}}^{r}\left(t, t_{2}\right) g_{\mathbf{k}^{\prime} \sigma^{\prime} \eta^{\prime}}^{<}\left(t_{2}, t^{\prime}\right) G_{j i_{1}}^{>}\left(t^{\prime}, t_{1}\right) g_{\mathbf{k} \sigma \eta}^{a}\left(t_{1}, t\right)\right] \text {. }
\end{aligned}
$$

Aplicando transformada de Fourier $S\left(\omega^{\prime}\right)=\int d\left(t-t^{\prime}\right) e^{-i \omega^{\prime}\left(t-t^{\prime}\right)} S\left(t-t^{\prime}\right)$ e tomando o 
limite $\omega^{\prime} \rightarrow 0$ vem

$$
\begin{aligned}
& S_{\eta \eta^{\prime}}\left(\omega^{\prime} \rightarrow 0\right)=e^{2} \sum_{\mathbf{k} \sigma i j} \frac{1}{2}(-1)^{(i+j) \delta_{\sigma \downarrow}}\left|t_{\mathbf{k} \sigma}\right|^{2} \int d \omega\left[g_{\mathbf{k} \sigma \eta}^{>}(\omega) G_{j i}^{<}(\omega)+g_{\mathbf{k} \sigma \eta}^{<}(\omega) G_{i j}^{>}(\omega)+\right. \\
& \left.g_{\mathbf{k} \sigma \eta}^{<}(\omega) G_{j i}^{>}(\omega)+g_{\mathbf{k} \sigma \eta}^{>}(\omega) G_{i j}^{<}(\omega)\right]- \\
& e^{2} \sum_{\mathbf{k} \mathbf{k}^{\prime} \sigma \sigma^{\prime}} \sum_{i j i_{1} i_{2}} \frac{1}{4}(-1)^{\left(i+i_{1}\right) \delta_{\sigma \downarrow}}(-1)^{\left(j+i_{2}\right) \delta_{\sigma^{\prime} \downarrow}\left|t_{\mathbf{k} \sigma}\right|^{2}\left|t_{\mathbf{k}^{\prime} \sigma^{\prime}}\right|^{2} \int d \omega \times} \\
& {\left[G_{i i_{2}}^{>}(\omega) g_{\mathbf{k}^{\prime} \sigma^{\prime} \eta^{\prime}}^{a}(\omega) G_{j i_{1}}^{r}(\omega) g_{\mathbf{k} \sigma \eta}^{<}(\omega)[1]\right.} \\
& +G_{i i_{2}}^{r}(\omega) g_{\mathbf{k}^{\prime} \sigma^{\prime} \eta^{\prime}}^{>}(\omega) G_{j i_{1}}^{r}(\omega) g_{\mathbf{k} \sigma \eta}^{<}(\omega) \\
& +G_{i i_{2}}^{>}(\omega) g_{\mathbf{k}^{\prime} \sigma^{\prime} \eta^{\prime}}^{a}(\omega) G_{j i_{1}}^{<}(\omega) g_{\mathbf{k} \sigma \eta}^{a}(\omega)[2] \\
& +G_{i i_{2}}^{r}(\omega) g_{\mathbf{k}^{\prime} \sigma^{\prime} \eta^{\prime}}^{>}(\omega) G_{j i_{1}}^{<}(\omega) g_{\mathbf{k} \sigma \eta}^{a}(\omega)[3] \\
& \text { - } G_{i j}^{>}(\omega) g_{\mathbf{k}^{\prime} \sigma^{\prime} \eta^{\prime}}^{r}(\omega) G_{i_{2} i_{1}}^{r}(\omega) g_{\mathbf{k} \sigma \eta}^{<}(\omega)[1] \\
& \text { - } G_{i j}^{>}(\omega) g_{\mathbf{k}^{\prime} \sigma^{\prime} \eta^{\prime}}^{r}(\omega) G_{i_{2} i_{1}}^{<}(\omega) g_{\mathbf{k} \sigma \eta}^{a}(\omega)[2] \\
& \text { - } G_{i j}^{>}(\omega) g_{\mathbf{k}^{\prime} \sigma^{\prime} \eta^{\prime}}^{<}(\omega) G_{i_{2} i_{1}}^{a}(\omega) g_{\mathbf{k} \sigma \eta}^{a}(\omega)[4] \\
& \text { - } g_{\mathbf{k} \sigma \eta}^{>}(\omega) G_{i_{1} i_{2}}^{a}(\omega) g_{\mathbf{k}^{\prime} \sigma^{\prime} \eta^{\prime}}^{a}\left(\omega^{\prime}\right) G_{j i}^{<}(\omega)[5] \\
& \text { - } g_{\mathbf{k} \sigma \eta}^{r}(\omega) G_{i_{1} i_{2}}^{>}(\omega) g_{\mathbf{k}^{\prime} \sigma^{\prime} \eta^{\prime}}^{a}\left(\omega^{\prime}\right) G_{j i}^{<}(\omega)[6] \\
& \text { - } g_{\mathbf{k} \sigma \eta}^{r}(\omega) G_{i_{1} i_{2}}^{r}(\omega) g_{\mathbf{k}^{\prime} \sigma^{\prime} \eta^{\prime}}^{>}\left(\omega^{\prime}\right) G_{j i}^{<}(\omega)[3] \\
& +g_{\mathbf{k} \sigma \eta}^{>}(\omega) G_{i_{1} j}^{a}(\omega) g_{\mathbf{k}^{\prime} \sigma^{\prime} \eta^{\prime}}^{r}(\omega) G_{i_{2} i}^{<}(\omega)[5] \\
& +g_{\mathbf{k} \sigma \eta}^{>}(\omega) G_{i_{1 j} j}^{a}(\omega) g_{\mathbf{k}^{\prime} \sigma^{\prime} \eta^{\prime}}^{<}(\omega) G_{i_{2} i}^{a}(\omega) \\
& +g_{\mathbf{k} \sigma \eta}^{r}(\omega) G_{i_{1} j}^{>}(\omega) g_{\mathbf{k}^{\prime} \sigma^{\prime} \eta^{\prime}}^{r}(\omega) G_{i_{2} i}^{<}(\omega)[6] \\
& +g_{\mathbf{k} \sigma \eta}^{r}(\omega) G_{i_{1} j}^{>}(\omega) g_{\mathbf{k}^{\prime} \sigma^{\prime} \eta^{\prime}}^{<}(\omega) G_{i_{2} i}^{a}(\omega)[4] \\
& +g_{\mathbf{k} \sigma \eta}^{<}(\omega) G_{i_{1} j}^{a}(\omega) g_{\mathbf{k}^{\prime} \sigma^{\prime} \eta^{\prime}}^{r}(\omega) G_{i_{2} i}^{>}(\omega)\left[3^{\prime}\right] \\
& +g_{\mathbf{k} \sigma \eta}^{<}(\omega) G_{i_{1} j}^{a}(\omega) g_{\mathbf{k}^{\prime} \sigma^{\prime} \eta^{\prime}}^{>}(\omega) G_{i_{2} i}^{a}(\omega) \\
& +g_{\mathbf{k} \sigma \eta}^{r}(\omega) G_{i_{1} j}^{<}(\omega) g_{\mathbf{k}^{\prime} \sigma^{\prime} \eta^{\prime}}^{r}(\omega) G_{i_{2} i}^{>}(\omega)\left[2^{\prime}\right] \\
& +g_{\mathbf{k} \sigma \eta}^{r}(\omega) G_{i_{1} j}^{<}(\omega) g_{\mathbf{k}^{\prime} \sigma^{\prime} \eta^{\prime}}^{>}(\omega) G_{i_{2} i}^{a}(\omega)\left[1^{\prime}\right] \\
& \text { - } g_{\mathbf{k} \sigma \eta}^{<}(\omega) G_{i_{1} i_{2}}^{a}(\omega) g_{\mathbf{k}^{\prime} \sigma^{\prime} \eta^{\prime}}^{a}\left(\omega^{\prime}\right) G_{j i}^{>}(\omega)\left[3^{\prime}\right] \\
& \text { - } g_{\mathbf{k} \sigma \eta}^{r}(\omega) G_{i_{1} i_{2}}^{<}(\omega) g_{\mathbf{k}^{\prime} \sigma^{\prime} \eta^{\prime}}^{a}\left(\omega^{\prime}\right) G_{j i}^{>}(\omega)\left[5^{\prime}\right] \\
& \text { - } g_{\mathbf{k} \sigma \eta}^{r}(\omega) G_{i_{1} i_{2}}^{r}(\omega) g_{\mathbf{k}^{\prime} \sigma^{\prime} \eta^{\prime}}^{<}\left(\omega^{\prime}\right) G_{j i}^{>}(\omega)\left[4^{\prime}\right] \\
& \text { - } G_{i j}^{<}(\omega) g_{\mathbf{k}^{\prime} \sigma^{\prime} \eta^{\prime}}^{r}(\omega) G_{i_{2} i_{1}}^{r}(\omega) g_{\mathbf{k} \sigma \eta}^{>}(\omega)\left[6^{\prime}\right] \\
& \text { - } G_{i j}^{<}(\omega) g_{\mathbf{k}^{\prime} \sigma^{\prime} \eta^{\prime}}^{r}(\omega) G_{i_{2} i_{1}}^{>}(\omega) g_{\mathbf{k} \sigma \eta}^{a}(\omega)\left[2^{\prime}\right] \\
& \text { - } G_{i j}^{<}(\omega) g_{\mathbf{k}^{\prime} \sigma^{\prime} \eta^{\prime}}^{>}(\omega) G_{i_{2} i_{1}}^{a}(\omega) g_{\mathbf{k} \sigma \eta}^{a}(\omega)\left[1^{\prime}\right] \\
& +G_{i i_{2}}^{<}(\omega) g_{\mathbf{k}^{\prime} \sigma^{\prime} \eta^{\prime}}^{a}(\omega) G_{j i_{1}}^{r}(\omega) g_{\mathbf{k} \sigma \eta}^{>}(\omega)\left[6^{\prime}\right] \\
& +G_{i i_{2}}^{r}(\omega) g_{\mathbf{k}^{\prime} \sigma^{\prime} \eta^{\prime}}^{<}(\omega) G_{j i_{1}}^{r}(\omega) g_{\mathbf{k} \sigma \eta}^{>}(\omega) \\
& +g_{\mathbf{k} \sigma \eta}^{a}(\omega) G_{j i_{1}}^{>}(\omega) g_{\mathbf{k}^{\prime} \sigma^{\prime} \eta^{\prime}}^{a}\left(\omega^{\prime}\right) G_{i i_{2}}^{<}(\omega)\left[5^{\prime}\right] \\
& \left.+g_{\mathbf{k} \sigma \eta}^{a}(\omega) G_{j i_{1}}^{>}(\omega) g_{\mathbf{k}^{\prime} \sigma^{\prime} \eta^{\prime}}^{<}\left(\omega^{\prime}\right) G_{i i_{2}}^{r}(\omega)\left[4^{\prime}\right]\right] .
\end{aligned}
$$




$$
\begin{aligned}
& S_{\eta \eta^{\prime}}\left(\omega^{\prime} \rightarrow 0\right)=2 e^{2} \sum_{\mathbf{k} \sigma i j} \frac{1}{2}(-1)^{(i+j) \delta_{\sigma \downarrow}}\left|t_{\mathbf{k} \sigma}\right|^{2} \int d \omega\left[g_{\mathbf{k} \sigma \eta}^{>}(\omega) G_{j i}^{<}(\omega)+g_{\mathbf{k} \sigma \eta}^{<}(\omega) G_{i j}^{>}(\omega)\right. \\
& -e^{2} \sum_{\mathbf{k k}^{\prime} \sigma \sigma^{\prime}} \sum_{i j i_{1} i_{2}} \frac{1}{4}(-1)^{\left(i+i_{1}\right) \delta_{\sigma \downarrow}}(-1)^{\left(j+i_{2}\right) \delta_{\sigma^{\prime} \downarrow}\left|t_{\mathbf{k} \sigma}\right|^{2}\left|t_{\mathbf{k}^{\prime} \sigma^{\prime}}\right|^{2} \int d \omega \times} \\
& \left\{G_{i j}^{>}(\omega)\left[g_{\mathbf{k}^{\prime} \sigma^{\prime} \eta^{\prime}}^{a}(\omega)-g_{\mathbf{k}^{\prime} \sigma^{\prime} \eta^{\prime}}^{r}(\omega)\right] G_{i_{2} i_{1}}^{r}(\omega) g_{\mathbf{k} \sigma \eta}^{<}(\omega)\right. \\
& +G_{i i_{2}}^{r}(\omega) g_{\mathbf{k}^{\prime} \sigma^{\prime} \eta^{\prime}}^{>}(\omega) G_{j i_{1}}^{r}(\omega) g_{\mathbf{k} \sigma \eta}^{<}(\omega) \\
& +[1] G_{i j}^{>}(\omega)\left[g_{\mathbf{k}^{\prime} \sigma^{\prime} \eta^{\prime}}^{a}(\omega)-g_{\mathbf{k}^{\prime} \sigma^{\prime} \eta^{\prime}}^{r}(\omega)\right] G_{i_{2} i_{1}}^{<}(\omega) g_{\mathbf{k} \sigma \eta}^{a}(\omega) \\
& +G_{i i_{2}}^{r}(\omega) g_{\mathbf{k}^{\prime} \sigma^{\prime} \eta^{\prime}}^{>}(\omega) G_{j i_{1}}^{<}(\omega)\left[g_{\mathbf{k} \sigma \eta}^{a}(\omega)-g_{\mathbf{k} \sigma \eta}^{r}(\omega)\right] \\
& \text { - } G_{i j}^{>}(\omega) g_{\mathbf{k}^{\prime} \sigma^{\prime} \eta^{\prime}}^{<}(\omega) G_{i_{2} i_{1}}^{a}(\omega)\left[g_{\mathbf{k} \sigma \eta}^{a}(\omega)-g_{\mathbf{k} \sigma \eta}^{r}(\omega)\right] \\
& \text { - } g_{\mathbf{k} \sigma \eta}^{>}(\omega) G_{i_{1} i_{2}}^{a}(\omega)\left[g_{\mathbf{k}^{\prime} \sigma^{\prime} \eta^{\prime}}^{a}\left(\omega^{\prime}\right)-g_{\mathbf{k}^{\prime} \sigma^{\prime} \eta^{\prime}}^{r}\left(\omega^{\prime}\right)\right] G_{j i}^{<}(\omega) \\
& \text { - }[1] g_{\mathbf{k} \sigma \eta}^{r}(\omega) G_{i_{1} i_{2}}^{>}(\omega)\left[g_{\mathbf{k}^{\prime} \sigma^{\prime} \eta^{\prime}}^{a}\left(\omega^{\prime}\right)-g_{\mathbf{k}^{\prime} \sigma^{\prime} \eta^{\prime}}^{r}\left(\omega^{\prime}\right)\right] G_{j i}^{<}(\omega) \\
& +g_{\mathbf{k} \sigma \eta}^{>}(\omega) G_{i_{1} j}^{a}(\omega) g_{\mathbf{k}^{\prime} \sigma^{\prime} \eta^{\prime}}^{<}(\omega) G_{i_{2} i}^{a}(\omega) \\
& \text { - } G_{i j}^{<}(\omega) g_{\mathbf{k}^{\prime} \sigma^{\prime} \eta^{\prime}}^{>}(\omega) G_{i_{2} i_{1}}^{a}(\omega)\left[g_{\mathbf{k} \sigma \eta}^{a}(\omega)-g_{\mathbf{k} \sigma \eta}^{r}(\omega)\right] \\
& \text { - } g_{\mathbf{k} \sigma \eta}^{<}(\omega) G_{i_{1} j}^{a}(\omega)\left[g_{\mathbf{k}^{\prime} \sigma^{\prime} \eta^{\prime}}^{a}(\omega)-g_{\mathbf{k}^{\prime} \sigma^{\prime} \eta^{\prime}}^{r}(\omega)\right] G_{i_{2} i}^{>}(\omega) \\
& +g_{\mathbf{k} \sigma \eta}^{<}(\omega) G_{i_{1} j}^{a}(\omega) g_{\mathbf{k}^{\prime} \sigma^{\prime} \eta^{\prime}}^{>}(\omega) G_{i_{2} i}^{a}(\omega) \\
& \text { - }[2] G_{i j}^{<}(\omega) g_{\mathbf{k}^{\prime} \sigma^{\prime} \eta^{\prime}}^{r}(\omega) G_{i_{2} i_{1}}^{>}(\omega)\left[g_{\mathbf{k} \sigma \eta}^{a}(\omega)-g_{\mathbf{k} \sigma \eta}^{r}(\omega)\right] \\
& +\left[g_{\mathbf{k} \sigma \eta}^{a}(\omega)-g_{\mathbf{k} \sigma \eta}^{r}(\omega)\right] G_{j i_{1}}^{>}(\omega) g_{\mathbf{k}^{\prime} \sigma^{\prime} \eta^{\prime}}^{<}\left(\omega^{\prime}\right) G_{i i_{2}}^{r}(\omega) \\
& +[2]\left[g_{\mathbf{k} \sigma \eta}^{a}(\omega)-g_{\mathbf{k} \sigma \eta}^{r}(\omega)\right] G_{j i_{1}}^{>}(\omega) g_{\mathbf{k}^{\prime} \sigma^{\prime} \eta^{\prime}}^{a}\left(\omega^{\prime}\right) G_{i i_{2}}^{<}(\omega) \\
& +G_{i i_{2}}^{<}(\omega)\left[g_{\mathbf{k}^{\prime} \sigma^{\prime} \eta^{\prime}}^{a}(\omega)-g_{\mathbf{k}^{\prime} \sigma^{\prime} \eta^{\prime}}^{r}(\omega)\right] G_{j i_{1}}^{r}(\omega) g_{\mathbf{k} \sigma \eta}^{>}(\omega) \\
& \left.+G_{i i_{2}}^{r}(\omega) g_{\mathbf{k}^{\prime} \sigma^{\prime} \eta^{\prime}}^{<}(\omega) G_{j i_{1}}^{r}(\omega) g_{\mathbf{k} \sigma \eta}^{>}(\omega)\right\} \text {. }
\end{aligned}
$$




$$
\begin{aligned}
& S_{\eta \eta^{\prime}}\left(\omega^{\prime} \rightarrow 0\right)=2 e^{2} \sum_{\mathbf{k} \sigma i j} \frac{1}{2}(-1)^{(i+j) \delta_{\sigma \downarrow}}\left|t_{\mathbf{k} \sigma}\right|^{2} \int d \omega\left[g_{\mathbf{k} \sigma \eta}^{>}(\omega) G_{j i}^{<}(\omega)+g_{\mathbf{k} \sigma \eta}^{<}(\omega) G_{i j}^{>}(\omega)\right. \\
& \text { - } e^{2} \sum_{\mathbf{k k}^{\prime} \sigma \sigma^{\prime}} \sum_{i j i_{1} i_{2}} \frac{1}{4}(-1)^{\left(i+i_{1}\right) \delta_{\sigma \downarrow}}(-1)^{\left(j+i_{2}\right) \delta_{\sigma^{\prime} \downarrow}\left|t_{\mathbf{k} \sigma}\right|^{2}\left|t_{\mathbf{k}^{\prime} \sigma^{\prime}}\right|^{2} \int d \omega \times} \\
& \left\{G_{i j}^{>}(\omega)\left[g_{\mathbf{k}^{\prime} \sigma^{\prime} \eta^{\prime}}^{a}(\omega)-g_{\mathbf{k}^{\prime} \sigma^{\prime} \eta^{\prime}}^{r}(\omega)\right] G_{i_{2} i_{1}}^{r}(\omega) g_{\mathbf{k} \sigma \eta}^{<}(\omega)\right. \\
& +G_{i i_{2}}^{r}(\omega) g_{\mathbf{k}^{\prime} \sigma^{\prime} \eta^{\prime}}^{>}(\omega) G_{j i_{1}}^{r}(\omega) g_{\mathbf{k} \sigma \eta}^{<}(\omega) \\
& +G_{i j}^{>}(\omega)\left[g_{\mathbf{k}^{\prime} \sigma^{\prime} \eta^{\prime}}^{a}(\omega)-g_{\mathbf{k}^{\prime} \sigma^{\prime} \eta^{\prime}}^{r}(\omega)\right] G_{i_{2} i_{1}}^{<}(\omega)\left[g_{\mathbf{k} \sigma \eta}^{a}(\omega)-g_{\mathbf{k} \sigma \eta}^{r}(\omega)\right] \\
& +G_{i i_{2}}^{r}(\omega) g_{\mathbf{k}^{\prime} \sigma^{\prime} \eta^{\prime}}^{>}(\omega) G_{j i_{1}}^{<}(\omega)\left[g_{\mathbf{k} \sigma \eta}^{a}(\omega)-g_{\mathbf{k} \sigma \eta}^{r}(\omega)\right] \\
& \text { - } G_{i j}^{>}(\omega) g_{\mathbf{k}^{\prime} \sigma^{\prime} \eta^{\prime}}^{<}(\omega) G_{i_{2} i_{1}}^{a}(\omega)\left[g_{\mathbf{k} \sigma \eta}^{a}(\omega)-g_{\mathbf{k} \sigma \eta}^{r}(\omega)\right] \\
& \text { - } g_{\mathbf{k} \sigma \eta}^{>}(\omega) G_{i_{1} i_{2}}^{a}(\omega)\left[g_{\mathbf{k}^{\prime} \sigma^{\prime} \eta^{\prime}}^{a}\left(\omega^{\prime}\right)-g_{\mathbf{k}^{\prime} \sigma^{\prime} \eta^{\prime}}^{r}\left(\omega^{\prime}\right)\right] G_{j i}^{<}(\omega) \\
& +g_{\mathbf{k} \sigma \eta}^{>}(\omega) G_{i_{1} j}^{a}(\omega) g_{\mathbf{k}^{\prime} \sigma^{\prime} \eta^{\prime}}^{<}(\omega) G_{i_{2} i}^{a}(\omega) \\
& \text { - } G_{i j}^{<}(\omega) g_{\mathbf{k}^{\prime} \sigma^{\prime} \eta^{\prime}}^{>}(\omega) G_{i_{2} i_{1}}^{a}(\omega)\left[g_{\mathbf{k} \sigma \eta}^{a}(\omega)-g_{\mathbf{k} \sigma \eta}^{r}(\omega)\right] \\
& \text { - } g_{\mathbf{k} \sigma \eta}^{<}(\omega) G_{i_{1} j}^{a}(\omega)\left[g_{\mathbf{k}^{\prime} \sigma^{\prime} \eta^{\prime}}^{a}(\omega)-g_{\mathbf{k}^{\prime} \sigma^{\prime} \eta^{\prime}}^{r}(\omega)\right] G_{i_{2} i}^{>}(\omega) \\
& +g_{\mathbf{k} \sigma \eta}^{<}(\omega) G_{i_{1 j} j}^{a}(\omega) g_{\mathbf{k}^{\prime} \sigma^{\prime} \eta^{\prime}}^{>}(\omega) G_{i_{2} i}^{a}(\omega) \\
& +\left[g_{\mathbf{k} \sigma \eta}^{a}(\omega)-g_{\mathbf{k} \sigma \eta}^{r}(\omega)\right] G_{j i_{1}}^{>}(\omega) g_{\mathbf{k}^{\prime} \sigma^{\prime} \eta^{\prime}}^{<}\left(\omega^{\prime}\right) G_{i i_{2}}^{r}(\omega) \\
& +\left[g_{\mathbf{k} \sigma \eta}^{a}(\omega)-g_{\mathbf{k} \sigma \eta}^{r}(\omega)\right] G_{j i_{1}}^{>}(\omega)\left[g_{\mathbf{k}^{\prime} \sigma^{\prime} \eta^{\prime}}^{a}\left(\omega^{\prime}\right)-g_{\mathbf{k}^{\prime} \sigma^{\prime} \eta^{\prime}}^{r}\left(\omega^{\prime}\right)\right] G_{i i_{2}}^{<}(\omega) \\
& +G_{i i_{2}}^{<}(\omega)\left[g_{\mathbf{k}^{\prime} \sigma^{\prime} \eta^{\prime}}^{a}(\omega)-g_{\mathbf{k}^{\prime} \sigma^{\prime} \eta^{\prime}}^{r}(\omega)\right] G_{j i_{1}}^{r}(\omega) g_{\mathbf{k} \sigma \eta}^{>}(\omega) \\
& \left.+G_{i i_{2}}^{r}(\omega) g_{\mathbf{k}^{\prime} \sigma^{\prime} \eta^{\prime}}^{<}(\omega) G_{j i_{1}}^{r}(\omega) g_{\mathbf{k} \sigma \eta}^{>}(\omega)\right\} \text {. }
\end{aligned}
$$

Usando o fato de que

$$
\begin{aligned}
g_{\mathbf{k} \sigma \eta}^{a}(\omega)-g_{\mathbf{k} \sigma \eta}^{r}(\omega) & =\frac{1}{\omega-\epsilon_{\mathbf{k} \sigma \eta}-i \delta}-\frac{1}{\omega-\epsilon_{\mathbf{k} \sigma \eta}+i \delta} \\
& =P \frac{1}{\omega-\epsilon_{\mathbf{k} \sigma \eta}}+i \pi \delta\left(\omega-\epsilon_{\mathbf{k} \sigma \eta}\right)-P \frac{1}{\omega-\epsilon_{\mathbf{k} \sigma \eta}}+i \pi \delta\left(\omega-\epsilon_{\mathbf{k} \sigma \eta}\right) \\
& =2 \pi i \delta\left(\omega-\epsilon_{\mathbf{k} \sigma \eta}\right)
\end{aligned}
$$




$$
\begin{aligned}
& g_{\mathbf{k} \sigma \eta}^{<}(\omega)=2 \pi i n_{\eta}(\omega) \delta\left(\omega-\epsilon_{\mathbf{k} \sigma \eta}\right), \text { e } g_{\mathbf{k} \sigma \eta}^{>}(\omega)=-2 \pi i\left[1-n_{\eta}(\omega)\right] \delta\left(\omega-\epsilon_{\mathbf{k} \sigma \eta}\right) \text {, vem } \\
& S_{\eta \eta^{\prime}}\left(\omega^{\prime} \rightarrow 0\right)=2 e^{2} \delta_{\eta \eta^{\prime}} \sum_{\mathbf{k} \sigma i j} \frac{1}{2}(-1)^{(i+j) \delta_{\sigma \downarrow}}\left|t_{\mathbf{k} \sigma}\right|^{2} \int d \omega \\
& {\left[-2 \pi i\left[1-n_{\eta}(\omega)\right] \delta\left(\omega-\epsilon_{\mathbf{k} \sigma \eta}\right) G_{j i}^{<}(\omega)+2 \pi i n_{\eta}(\omega) \delta\left(\omega-\epsilon_{\mathbf{k} \sigma \eta}\right) G_{i j}^{>}(\omega)\right.} \\
& \text { - } e^{2} \sum_{\mathbf{k} \mathbf{k}^{\prime} \sigma \sigma^{\prime}} \sum_{i j i_{1} i_{2}} \frac{1}{4}(-1)^{\left(i+i_{1}\right) \delta_{\sigma \downarrow}}(-1)^{\left(j+i_{2}\right) \delta_{\sigma^{\prime} \downarrow}\left|t_{\mathbf{k} \sigma}\right|^{2}\left|t_{\mathbf{k}^{\prime} \sigma^{\prime}}\right|^{2} \int d \omega \times} \\
& \left\{G_{i j}^{>}(\omega) 2 \pi i \delta\left(\omega-\epsilon_{\mathbf{k}^{\prime} \sigma^{\prime} \eta^{\prime}}\right) G_{i_{2} i_{1}}^{r}(\omega) 2 \pi i n_{\eta}(\omega) \delta\left(\omega-\epsilon_{\mathbf{k} \sigma \eta}\right)\right. \\
& \text { - } G_{i i_{2}}^{r}(\omega) 2 \pi i\left[1-n_{\eta^{\prime}}(\omega)\right] \delta\left(\omega-\epsilon_{\mathbf{k}^{\prime} \sigma^{\prime} \eta^{\prime}}\right) G_{j i_{1}}^{r}(\omega) 2 \pi i n_{\eta}(\omega) \delta\left(\omega-\epsilon_{\mathbf{k} \sigma \eta}\right) \\
& +G_{i j}^{>}(\omega) 2 \pi i \delta\left(\omega-\epsilon_{\mathbf{k}^{\prime} \sigma^{\prime} \eta^{\prime}}\right) G_{i_{2} i_{1}}^{<}(\omega) 2 \pi i \delta\left(\omega-\epsilon_{\mathbf{k} \sigma \eta}\right) \\
& \text { - } G_{i i_{2}}^{r}(\omega) 2 \pi i\left[1-n_{\eta^{\prime}}(\omega)\right] \delta\left(\omega-\epsilon_{\mathbf{k}^{\prime} \sigma^{\prime} \eta^{\prime}}\right) G_{j i_{1}}^{<}(\omega) 2 \pi i \delta\left(\omega-\epsilon_{\mathbf{k} \sigma \eta}\right) \\
& \text { - } G_{i j}^{>}(\omega) 2 \pi i n_{\eta^{\prime}}(\omega) \delta\left(\omega-\epsilon_{\mathbf{k}^{\prime} \sigma^{\prime} \eta^{\prime}}\right) G_{i_{2} i_{1}}^{a}(\omega) 2 \pi i \delta\left(\omega-\epsilon_{\mathbf{k} \sigma \eta}\right) \\
& +2 \pi i\left[1-n_{\eta}(\omega)\right] \delta\left(\omega-\epsilon_{\mathbf{k} \sigma \eta}\right) G_{i_{1} i_{2}}^{a}(\omega) 2 \pi i \delta\left(\omega-\epsilon_{\mathbf{k}^{\prime} \sigma^{\prime} \eta^{\prime}}\right) G_{j i}^{<}(\omega) \\
& \text { - } 2 \pi i\left[1-n_{\eta}(\omega)\right] \delta\left(\omega-\epsilon_{\mathbf{k} \sigma \eta}\right) G_{i_{1 j} j}^{a}(\omega) 2 \pi i n_{\eta^{\prime}}(\omega) \delta\left(\omega-\epsilon_{\mathbf{k}^{\prime} \sigma^{\prime} \eta^{\prime}}\right) G_{i_{2} i}^{a}(\omega) \\
& +G_{i j}^{<}(\omega) 2 \pi i\left[1-n_{\eta^{\prime}}(\omega)\right] \delta\left(\omega-\epsilon_{\mathbf{k}^{\prime} \sigma^{\prime} \eta^{\prime}}\right) G_{i_{2} i_{1}}^{a}(\omega) 2 \pi i \delta\left(\omega-\epsilon_{\mathbf{k} \sigma \eta}\right) \\
& \text { - } 2 \pi i n_{\eta}(\omega) \delta\left(\omega-\epsilon_{\mathbf{k} \sigma \eta}\right) G_{i_{1} j}^{a}(\omega) 2 \pi i \delta\left(\omega-\epsilon_{\mathbf{k}^{\prime} \sigma^{\prime} \eta^{\prime}}\right) G_{i_{2} i}^{>}(\omega) \\
& \text { - } 2 \pi i n_{\eta}(\omega) \delta\left(\omega-\epsilon_{\mathbf{k} \sigma \eta}\right) G_{i_{1} j}^{a}(\omega) 2 \pi i\left[1-n_{\eta^{\prime}}(\omega)\right] \delta\left(\omega-\epsilon_{\mathbf{k}^{\prime} \sigma^{\prime} \eta^{\prime}}\right) G_{i_{2} i}^{a}(\omega) \\
& +2 \pi i \delta\left(\omega-\epsilon_{\mathbf{k} \sigma \eta}\right) G_{j i_{1}}^{>}(\omega) 2 \pi i n_{\eta^{\prime}}(\omega) \delta\left(\omega-\epsilon_{\mathbf{k}^{\prime} \sigma^{\prime} \eta^{\prime}}\right) G_{i i_{2}}^{r}(\omega) \\
& +2 \pi i \delta\left(\omega-\epsilon_{\mathbf{k} \sigma \eta}\right) G_{j i_{1}}^{>}(\omega) 2 \pi i \delta\left(\omega-\epsilon_{\mathbf{k}^{\prime} \sigma^{\prime} \eta^{\prime}}\right) G_{i i_{2}}^{<}(\omega) \\
& \text { - } G_{i i_{2}}^{<}(\omega) 2 \pi i \delta\left(\omega-\epsilon_{\mathbf{k}^{\prime} \sigma^{\prime} \eta^{\prime}}\right) G_{j i_{1}}^{r}(\omega) 2 \pi i\left[1-n_{\eta}(\omega)\right] \delta\left(\omega-\epsilon_{\mathbf{k} \sigma \eta}\right) \\
& \text { - } \left.G_{i i_{2}}^{r}(\omega) 2 \pi i n_{\eta^{\prime}}(\omega) \delta\left(\omega-\epsilon_{\mathbf{k}^{\prime} \sigma^{\prime} \eta^{\prime}}\right) G_{j i_{1}}^{r}(\omega) 2 \pi i\left[1-n_{\eta}(\omega)\right] \delta\left(\omega-\epsilon_{\mathbf{k} \sigma \eta}\right)\right\} \text {. }
\end{aligned}
$$


Usando a definição $\Gamma_{\sigma}^{\eta}=2 \pi \sum_{\mathbf{k}}\left|t_{\mathbf{k} \sigma}\right|^{2} \delta\left(\epsilon-\epsilon_{\mathbf{k} \sigma \eta}\right)$

$$
\begin{aligned}
S_{\eta \eta^{\prime}}\left(\omega^{\prime} \rightarrow 0\right)= & 2 e^{2} \delta_{\eta \eta^{\prime}} \sum_{\sigma i j} \frac{1}{2}(-1)^{(i+j) \delta_{\sigma \downarrow}} \int d \omega \\
& {\left[-i\left[1-n_{\eta}(\omega)\right] \Gamma_{\sigma}^{\eta} G_{j i}^{<}(\omega)+i n_{\eta}(\omega) \Gamma_{\sigma}^{\eta} G_{i j}^{>}(\omega)\right] } \\
+ & e^{2} \sum_{\sigma \sigma^{\prime}} \sum_{i j i_{1} i_{2}} \frac{1}{4}(-1)^{\left(i+i_{1}\right) \delta_{\sigma \downarrow}}(-1)^{\left(j+i_{2}\right) \delta_{\sigma^{\prime} \downarrow} \int d \omega \times} \\
& \left\{G_{i j}^{>}(\omega) \Gamma_{\sigma^{\prime}}^{\eta^{\prime}} G_{i_{2} i_{1}}^{r}(\omega) n_{\eta}(\omega) \Gamma_{\sigma}^{\eta}\right. \\
- & G_{i i_{2}}^{r}(\omega)\left[1-n_{\eta^{\prime}}(\omega)\right] \Gamma_{\sigma^{\prime}}^{\eta^{\prime}} G_{j i_{1}}^{r}(\omega) n_{\eta}(\omega) \Gamma_{\sigma}^{\eta} \\
+ & G_{i j}^{>}(\omega) \Gamma_{\sigma^{\prime}}^{\eta^{\prime}} G_{i_{2} i_{1}}^{<}(\omega) \Gamma_{\sigma}^{\eta} \\
- & G_{i i_{2}}^{r}(\omega)\left[1-n_{\eta^{\prime}}(\omega)\right] \Gamma_{\sigma^{\prime}}^{\eta^{\prime}} G_{j i_{1}}^{<}(\omega) \Gamma_{\sigma}^{\eta} \\
- & G_{i j}^{>}(\omega) n_{\eta^{\prime}}(\omega) \Gamma_{\sigma^{\prime}}^{\eta^{\prime}} G_{i_{2} i_{1}}^{a}(\omega) \Gamma_{\sigma}^{\eta} \\
+ & {\left[1-n_{\eta}(\omega)\right] \Gamma_{\sigma}^{\eta} G_{i_{1} i_{2}}^{a}(\omega) \Gamma_{\sigma^{\prime}}^{\eta^{\prime}} G_{j i}^{<}(\omega) } \\
- & {\left[1-n_{\eta}(\omega)\right] \Gamma_{\sigma}^{\eta} G_{i_{1} j}^{a}(\omega) n_{\eta^{\prime}}(\omega) \Gamma_{\sigma^{\prime}}^{\eta^{\prime}} G_{i_{2} i}^{a}(\omega) } \\
+ & G_{i j}^{<}(\omega)\left[1-n_{\eta^{\prime}}(\omega)\right] \Gamma_{\sigma^{\prime}}^{\eta^{\prime}} G_{i_{2} i_{1}}^{a}(\omega) \Gamma_{\sigma}^{\eta} \\
& -n_{\eta}(\omega) \Gamma_{\sigma}^{\eta} G_{i_{1} j}^{a}(\omega) \Gamma_{\sigma^{\prime}}^{\eta^{\prime}} G_{i_{2} i}^{>}(\omega) \\
& -n_{\eta}(\omega) \Gamma_{\sigma}^{\eta} G_{i_{1} j}^{a}(\omega)\left[1-n_{\eta^{\prime}}(\omega)\right] \Gamma_{\sigma^{\prime}}^{\eta^{\prime}} G_{i_{2} i}^{a}(\omega) \\
+ & \Gamma_{\sigma}^{\eta} G_{j i_{1}}^{>}(\omega) n_{\eta^{\prime}}(\omega) \Gamma_{\sigma^{\prime}}^{\eta^{\prime}} G_{i i_{2}}^{r}(\omega) \\
+ & \Gamma_{\sigma}^{\eta} G_{j i_{1}}^{>}(\omega) \Gamma_{\sigma^{\prime}}^{\eta^{\prime}} G_{i i_{2}}^{<}(\omega) \\
- & G_{i i_{2}}^{<}(\omega) \Gamma_{\sigma^{\prime}}^{\eta^{\prime}} G_{j i_{1}}^{r}(\omega)\left[1-n_{\eta}(\omega)\right] \Gamma_{\sigma}^{\eta} \\
- & \left.G_{i i_{2}}^{r}(\omega) n_{\eta^{\prime}}(\omega) \Gamma_{\sigma^{\prime}}^{\eta^{\prime}} G_{j i_{1}}^{r}(\omega)\left[1-n_{\eta}(\omega)\right] \Gamma_{\sigma}^{\eta}\right\} \\
&
\end{aligned}
$$


Usando que $\Gamma_{j i}^{\eta}=(1 / 2) \sum_{\sigma}(-1)^{(i+j) \delta_{\sigma \downarrow}} \Gamma_{\sigma}^{\eta}$

$$
\begin{aligned}
& S_{\eta \eta^{\prime}}\left(\omega^{\prime} \rightarrow 0\right)= 2 e^{2} \delta_{\eta \eta^{\prime}} \sum_{i j} \int d \omega\left[-i\left[1-n_{\eta}(\omega)\right] \Gamma_{i j}^{\eta} G_{j i}^{<}(\omega)+i n_{\eta}(\omega) \Gamma_{j i}^{\eta} G_{i j}^{>}(\omega)\right. \\
&+ e^{2} \sum_{i j i_{1} i_{2}} \int d \omega \times \\
&\left\{G_{i j}^{>}(\omega) \Gamma_{j i_{2}}^{\eta^{\prime}} G_{i_{2} i_{1}}^{r}(\omega) n_{\eta}(\omega) \Gamma_{i_{1} i}^{\eta}\right. \\
&- G_{i i_{2}}^{r}(\omega)\left[1-n_{\eta^{\prime}}(\omega)\right] \Gamma_{i_{2} j}^{\eta^{\prime}} G_{j i_{1}}^{r}(\omega) n_{\eta}(\omega) \Gamma_{i_{1} i}^{\eta} \\
&+ G_{i j}^{>}(\omega) \Gamma_{j i_{2}}^{\eta^{\prime}} G_{i_{2} i_{1}}^{<}(\omega) \Gamma_{i_{1} i}^{\eta} \\
&- G_{i i_{2}}^{r}(\omega)\left[1-n_{\eta^{\prime}}(\omega)\right] \Gamma_{i_{2} j}^{\eta^{\prime}} G_{j i_{1}}^{<}(\omega) \Gamma_{i_{1} i}^{\eta} \\
&- G_{i j}^{>}(\omega) n_{\eta^{\prime}}(\omega) \Gamma_{j i_{2}}^{\eta^{\prime}} G_{i_{2} i_{1}}^{a}(\omega) \Gamma_{i_{1} i}^{\eta} \\
&+ {\left[1-n_{\eta}(\omega)\right] \Gamma_{i i_{1}}^{\eta} G_{i_{1} i_{2}}^{a}(\omega) \Gamma_{i_{2} j}^{\eta^{\prime}} G_{j i}^{<}(\omega) } \\
&- {\left[1-n_{\eta}(\omega)\right] \Gamma_{i i_{1}}^{\eta} G_{i_{1} j}^{a}(\omega) n_{\eta^{\prime}}(\omega) \Gamma_{j i_{2}}^{\eta^{\prime}} G_{i_{2} i}^{a}(\omega) } \\
&+ G_{i j}^{<}(\omega)\left[1-n_{\eta^{\prime}}(\omega)\right] \Gamma_{j i_{2}}^{\eta^{\prime}} G_{i_{2} i_{1}}^{a}(\omega) \Gamma_{i_{1} i}^{\eta} \\
&- n_{\eta}(\omega) \Gamma_{i i_{1}}^{\eta} G_{i_{1} j}^{a}(\omega) \Gamma_{j i_{2}}^{\eta^{\prime}} G_{i_{2} i}^{>}(\omega) \\
&- n_{\eta}(\omega) \Gamma_{i i_{1}}^{\eta} G_{i_{1} j}^{a}(\omega)\left[1-n_{\eta^{\prime}}(\omega)\right] \Gamma_{j i_{2}}^{\eta^{\prime}} G_{i_{2} i}^{a}(\omega) \\
&+ \Gamma_{i_{2} j}^{\eta^{\prime}} G_{j i_{1}}^{>}(\omega) n_{\eta^{\prime}}(\omega) \Gamma_{i_{1} i}^{\eta} G_{i i_{2}}^{r}(\omega) \\
&+ \Gamma_{i_{2} j}^{\eta^{\prime}} G_{j i_{1}}^{>}(\omega) \Gamma_{i_{1} i}^{\eta} G_{i i_{2}}^{<}(\omega) \\
&- G_{i i_{2}}^{<}(\omega) \Gamma_{i_{2} j}^{\eta^{\prime}} G_{j i_{1}}^{r}(\omega)\left[1-n_{\eta}(\omega)\right] \Gamma_{i_{1} i}^{\eta} \\
&-\left.G_{i i_{2}}^{r}(\omega) n_{\eta^{\prime}}(\omega) \Gamma_{i_{2} j}^{\eta^{\prime}} G_{j i_{1}}^{r}(\omega)\left[1-n_{\eta}(\omega)\right] \Gamma_{i_{1} i}^{\eta}\right\} \\
&
\end{aligned}
$$

Reescrevendo essa equação utilizando notação matricial vem

$$
\begin{aligned}
S_{\eta \eta^{\prime}}\left(\omega^{\prime} \rightarrow 0\right)= & 2 e^{2} \delta_{\eta \eta^{\prime}} \int d \omega \operatorname{Tr}\left[-i\left[1-n_{\eta}(\omega)\right] \boldsymbol{\Gamma}^{\eta} \mathbf{G}^{<}(\omega)+i n_{\eta}(\omega) \boldsymbol{\Gamma}^{\eta} \mathbf{G}^{>}(\omega)\right. \\
+ & e^{2} \int d \omega \operatorname{Tr} \times \\
& \left\{n_{\eta}(\omega) \mathbf{G}^{>}(\omega) \boldsymbol{\Gamma}^{\eta^{\prime}} \mathbf{G}^{r}(\omega) \boldsymbol{\Gamma}^{\eta}\right. \\
- & n_{\eta}(\omega)\left[1-n_{\eta^{\prime}}(\omega)\right] \mathbf{G}^{r}(\omega) \boldsymbol{\Gamma}^{\eta^{\prime}} \mathbf{G}^{r}(\omega) \boldsymbol{\Gamma}^{\eta} \\
+ & \mathbf{G}^{>}(\omega) \boldsymbol{\Gamma}^{\eta^{\prime}} \mathbf{G}^{<}(\omega) \boldsymbol{\Gamma}^{\eta} \\
- & {\left[1-n_{\eta^{\prime}}(\omega)\right] \mathbf{G}^{r}(\omega) \boldsymbol{\Gamma}^{\eta^{\prime}} \mathbf{G}^{<}(\omega) \boldsymbol{\Gamma}^{\eta} } \\
- & n_{\eta^{\prime}}(\omega) \mathbf{G}^{>}(\omega) \boldsymbol{\Gamma}^{\eta^{\prime}} \mathbf{G}^{a}(\omega) \boldsymbol{\Gamma}^{\eta} \\
+ & {\left[1-n_{\eta}(\omega)\right] \boldsymbol{\Gamma}^{\eta} \mathbf{G}^{a}(\omega) \boldsymbol{\Gamma}^{\eta^{\prime}} \mathbf{G}^{<}(\omega) } \\
- & n_{\eta^{\prime}}(\omega)\left[1-n_{\eta}(\omega)\right] \boldsymbol{\Gamma}^{\eta} \mathbf{G}^{a}(\omega) \boldsymbol{\Gamma}^{\eta^{\prime}} \mathbf{G}^{a}(\omega) \\
+ & {\left[1-n_{\eta^{\prime}}(\omega)\right] \mathbf{G}^{<}(\omega) \boldsymbol{\Gamma}^{\eta^{\prime}} \mathbf{G}^{a}(\omega) \boldsymbol{\Gamma}^{\eta} } \\
- & n_{\eta}(\omega) \boldsymbol{\Gamma}^{\eta} \mathbf{G}^{a}(\omega) \boldsymbol{\Gamma}^{\eta^{\prime}} \mathbf{G}^{>}(\omega) \\
- & n_{\eta}(\omega)\left[1-n_{\eta^{\prime}}(\omega)\right] \boldsymbol{\Gamma}^{\eta} \mathbf{G}^{a}(\omega) \boldsymbol{\Gamma}^{\eta^{\prime}} \mathbf{G}^{a}(\omega) \\
+ & n_{\eta^{\prime}}(\omega) \boldsymbol{\Gamma}^{\eta^{\prime}} \mathbf{G}^{>}(\omega) \boldsymbol{\Gamma}^{\eta} \mathbf{G}^{r}(\omega) \\
+ & \boldsymbol{\Gamma}^{\eta^{\prime}} \mathbf{G}^{>}(\omega) \boldsymbol{\Gamma}^{\eta} \mathbf{G}^{<}(\omega) \\
- & {\left[1-n_{\eta}(\omega)\right] \mathbf{G}^{<}(\omega) \boldsymbol{\Gamma}^{\eta^{\prime}} \mathbf{G}^{r}(\omega) \boldsymbol{\Gamma}^{\eta} } \\
- & \left.n_{\eta^{\prime}}(\omega)\left[1-n_{\eta}(\omega)\right] \mathbf{G}^{r}(\omega) \boldsymbol{\Gamma}^{\eta^{\prime}} \mathbf{G}^{r}(\omega) \boldsymbol{\Gamma}^{\eta}\right\}
\end{aligned}
$$


Reagrupando termos vem

$$
\begin{aligned}
S_{\eta \eta^{\prime}}(\omega \rightarrow 0) & =e^{2} \int \frac{d \omega^{\prime}}{2 \pi} \operatorname{Tr}\left\{\left\{\delta_{\eta \eta^{\prime}} 2 i n_{\eta} \boldsymbol{\Gamma}^{\eta} \mathbf{G}^{>}-\delta_{\eta \eta^{\prime}} 2 i\left[1-n_{\eta}\right] \boldsymbol{\Gamma}^{\eta} \mathbf{G}^{<}\right\}\right. \\
& +n_{\eta}\left(\mathbf{G}^{>} \boldsymbol{\Gamma}^{\eta^{\prime}} \mathbf{G}^{r} \boldsymbol{\Gamma}^{\eta}-\mathbf{G}^{>} \boldsymbol{\Gamma}^{\eta} \mathbf{G}^{a} \boldsymbol{\Gamma}^{\eta^{\prime}}\right)+n_{\eta^{\prime}}\left(\mathbf{G}^{>} \boldsymbol{\Gamma}^{\eta} \mathbf{G}^{r} \boldsymbol{\Gamma}^{\eta^{\prime}}-\mathbf{G}^{>} \boldsymbol{\Gamma}^{\eta^{\prime}} \mathbf{G}^{a} \boldsymbol{\Gamma}^{\eta}\right) \\
& +\left[1-n_{\eta}\right]\left(\mathbf{G}^{a} \boldsymbol{\Gamma}^{\eta^{\prime}} \mathbf{G}^{<} \boldsymbol{\Gamma}^{\eta}-\mathbf{G}^{r} \boldsymbol{\Gamma}^{\eta} \mathbf{G}^{<} \boldsymbol{\Gamma}^{\eta^{\prime}}\right) \\
& +\left[1-n_{\eta^{\prime}}\right]\left(\mathbf{G}^{a} \boldsymbol{\Gamma}^{\eta} \mathbf{G}^{<} \boldsymbol{\Gamma}^{\eta^{\prime}}-\mathbf{G}^{r} \boldsymbol{\Gamma}^{\eta^{\prime}} \mathbf{G}^{<} \boldsymbol{\Gamma}^{\eta}\right) \\
& -n_{\eta^{\prime}}\left[1-n_{\eta}\right]\left(\mathbf{G}^{a} \boldsymbol{\Gamma}^{\eta^{\prime}} \mathbf{G}^{a} \boldsymbol{\Gamma}^{\eta}+\mathbf{G}^{r} \boldsymbol{\Gamma}^{\eta^{\prime}} \mathbf{G}^{r} \boldsymbol{\Gamma}^{\eta}\right) \\
& -\left[1-n_{\eta^{\prime}}\right] n_{\eta}\left(\mathbf{G}^{a} \boldsymbol{\Gamma}^{\eta} \mathbf{G}^{a} \boldsymbol{\Gamma}^{\eta^{\prime}}+\mathbf{G}^{r} \boldsymbol{\Gamma}^{\eta} \mathbf{G}^{r} \boldsymbol{\Gamma}^{\eta^{\prime}}\right) \\
& \left.\left.+\mathbf{G}^{<} \boldsymbol{\Gamma}^{\eta^{\prime}} \mathbf{G}^{>} \boldsymbol{\Gamma}^{\eta}+\mathbf{G}^{<} \boldsymbol{\Gamma}^{\eta} \mathbf{G}^{>} \boldsymbol{\Gamma}^{\eta^{\prime}}\right\}\right\} .
\end{aligned}
$$

Dessa equação obtemos as várias componentes do ruído. Para $\eta=\eta^{\prime}=L$ temos

$$
\begin{aligned}
S_{L L}(\omega \rightarrow 0) & =2 e^{2} \int \frac{d \omega^{\prime}}{2 \pi} \operatorname{Tr}\left\{\left\{i n_{L} \boldsymbol{\Gamma}^{L} \mathbf{G}^{>}-i\left[1-n_{L}\right] \boldsymbol{\Gamma}^{L} \mathbf{G}^{<}\right\}\right. \\
& +n_{L}\left(\mathbf{G}^{>} \boldsymbol{\Gamma}^{L} \mathbf{G}^{r} \boldsymbol{\Gamma}^{L}-\mathbf{G}^{>} \boldsymbol{\Gamma}^{L} \mathbf{G}^{a} \boldsymbol{\Gamma}^{L}\right)+\left[1-n_{L}\right]\left(\mathbf{G}^{a} \boldsymbol{\Gamma}^{L} \mathbf{G}^{<} \boldsymbol{\Gamma}^{L}-\mathbf{G}^{r} \boldsymbol{\Gamma}^{L} \mathbf{G}^{<} \boldsymbol{\Gamma}^{L}\right) \\
& \left.-n_{L}\left[1-n_{L}\right]\left(\mathbf{G}^{a} \boldsymbol{\Gamma}^{L} \mathbf{G}^{a} \boldsymbol{\Gamma}^{L}+\mathbf{G}^{r} \boldsymbol{\Gamma}^{L} \mathbf{G}^{r} \boldsymbol{\Gamma}^{L}\right)+\mathbf{G}^{<} \boldsymbol{\Gamma}^{L} \mathbf{G}^{>} \boldsymbol{\Gamma}^{L}\right\},
\end{aligned}
$$

que também pode ser escrita como

$$
\begin{aligned}
S_{L L}(\omega \rightarrow 0) & =2 e^{2} \int \frac{d \omega^{\prime}}{2 \pi} \operatorname{Tr}\left\{\left\{i n_{L} \boldsymbol{\Gamma}^{L} \mathbf{G}^{>}-i\left[1-n_{L}\right] \boldsymbol{\Gamma}^{L} \mathbf{G}^{<}\right\}\right. \\
& +\left(\mathbf{G}^{r}-\mathbf{G}^{a}\right)\left(n_{L} \boldsymbol{\Gamma}^{L} \mathbf{G}^{>} \boldsymbol{\Gamma}^{L}-\left[1-n_{L}\right] \boldsymbol{\Gamma}^{L} \mathbf{G}^{<} \boldsymbol{\Gamma}^{L}\right) \\
& \left.-n_{L}\left[1-n_{L}\right]\left(\mathbf{G}^{a} \boldsymbol{\Gamma}^{L} \mathbf{G}^{a} \boldsymbol{\Gamma}^{L}+\mathbf{G}^{r} \boldsymbol{\Gamma}^{L} \mathbf{G}^{r} \boldsymbol{\Gamma}^{L}\right)+\mathbf{G}^{<} \boldsymbol{\Gamma}^{L} \mathbf{G}^{>} \boldsymbol{\Gamma}^{L}\right\} .
\end{aligned}
$$

Para a componente da direita temos um resultado semelhante

$$
\begin{aligned}
S_{R R}(\omega \rightarrow 0) & =2 e^{2} \int \frac{d \omega^{\prime}}{2 \pi} \operatorname{Tr}\left\{\left\{i n_{R} \boldsymbol{\Gamma}^{R} \mathbf{G}^{>}-i\left[1-n_{R}\right] \boldsymbol{\Gamma}^{R} \mathbf{G}^{<}\right\}\right. \\
& +\left(\mathbf{G}^{r}-\mathbf{G}^{a}\right)\left(n_{R} \boldsymbol{\Gamma}^{R} \mathbf{G}^{>} \boldsymbol{\Gamma}^{R}-\left[1-n_{R}\right] \boldsymbol{\Gamma}^{R} \mathbf{G}^{<} \boldsymbol{\Gamma}^{R}\right) \\
& \left.-n_{R}\left[1-n_{R}\right]\left(\mathbf{G}^{a} \boldsymbol{\Gamma}^{R} \mathbf{G}^{a} \boldsymbol{\Gamma}^{R}+\mathbf{G}^{r} \boldsymbol{\Gamma}^{R} \mathbf{G}^{r} \boldsymbol{\Gamma}^{R}\right)+\mathbf{G}^{<} \boldsymbol{\Gamma}^{R} \mathbf{G}^{>} \boldsymbol{\Gamma}^{R}\right\}
\end{aligned}
$$

e para a componente $S_{L R}$ nós temos

$$
\begin{aligned}
S_{L R}(\omega \rightarrow 0) & =2 e^{2} \int \frac{d \omega^{\prime}}{2 \pi} \operatorname{Tr}\{ \\
& +n_{L}\left(\mathbf{G}^{>} \boldsymbol{\Gamma}^{R} \mathbf{G}^{r} \boldsymbol{\Gamma}^{L}-\mathbf{G}^{>} \boldsymbol{\Gamma}^{L} \mathbf{G}^{a} \boldsymbol{\Gamma}^{R}\right)+n_{R}\left(\mathbf{G}^{>} \boldsymbol{\Gamma}^{L} \mathbf{G}^{r} \boldsymbol{\Gamma}^{R}-\mathbf{G}^{>} \boldsymbol{\Gamma}^{R} \mathbf{G}^{a} \boldsymbol{\Gamma}^{L}\right) \\
& +\left[1-n_{L}\right]\left(\mathbf{G}^{a} \boldsymbol{\Gamma}^{R} \mathbf{G}^{<} \boldsymbol{\Gamma}^{L}-\mathbf{G}^{r} \boldsymbol{\Gamma}^{L} \mathbf{G}^{<} \boldsymbol{\Gamma}^{R}\right) \\
& +\left[1-n_{R}\right]\left(\mathbf{G}^{a} \boldsymbol{\Gamma}^{L} \mathbf{G}^{<} \boldsymbol{\Gamma}^{R}-\mathbf{G}^{r} \boldsymbol{\Gamma}^{R} \mathbf{G}^{<} \boldsymbol{\Gamma}^{L}\right) \\
& -n_{R}\left[1-n_{L}\right]\left(\mathbf{G}^{a} \boldsymbol{\Gamma}^{R} \mathbf{G}^{a} \boldsymbol{\Gamma}^{L}+\mathbf{G}^{r} \boldsymbol{\Gamma}^{R} \mathbf{G}^{r} \boldsymbol{\Gamma}^{L}\right) \\
& -\left[1-n_{R}\right] n_{L}\left(\mathbf{G}^{a} \boldsymbol{\Gamma}^{L} \mathbf{G}^{a} \boldsymbol{\Gamma}^{R}+\mathbf{G}^{r} \boldsymbol{\Gamma}^{L} \mathbf{G}^{r} \boldsymbol{\Gamma}^{R}\right) \\
& \left.\left.+\mathbf{G}^{<} \boldsymbol{\Gamma}^{R} \mathbf{G}^{>} \boldsymbol{\Gamma}^{L}+\mathbf{G}^{<} \boldsymbol{\Gamma}^{L} \mathbf{G}^{>} \boldsymbol{\Gamma}^{R}\right\}\right\} .
\end{aligned}
$$


E para $S_{R L}$ vem

$$
\begin{aligned}
S_{R L}(\omega \rightarrow 0) & =2 e^{2} \int \frac{d \omega^{\prime}}{2 \pi} \operatorname{Tr}\{ \\
& +n_{R}\left(\mathbf{G}^{>} \boldsymbol{\Gamma}^{L} \mathbf{G}^{r} \boldsymbol{\Gamma}^{R}-\mathbf{G}^{>} \boldsymbol{\Gamma}^{R} \mathbf{G}^{a} \boldsymbol{\Gamma}^{L}\right)+n_{L}\left(\mathbf{G}^{>} \boldsymbol{\Gamma}^{R} \mathbf{G}^{r} \boldsymbol{\Gamma}^{L}-\mathbf{G}^{>} \boldsymbol{\Gamma}^{L} \mathbf{G}^{a} \boldsymbol{\Gamma}^{R}\right) \\
& +\left[1-n_{R}\right]\left(\mathbf{G}^{a} \boldsymbol{\Gamma}^{L} \mathbf{G}^{<} \boldsymbol{\Gamma}^{R}-\mathbf{G}^{r} \boldsymbol{\Gamma}^{R} \mathbf{G}^{<} \boldsymbol{\Gamma}^{L}\right) \\
& +\left[1-n_{L}\right]\left(\mathbf{G}^{a} \boldsymbol{\Gamma}^{R} \mathbf{G}^{<} \boldsymbol{\Gamma}^{L}-\mathbf{G}^{r} \boldsymbol{\Gamma}^{L} \mathbf{G}^{<} \boldsymbol{\Gamma}^{R}\right) \\
& -n_{L}\left[1-n_{R}\right]\left(\mathbf{G}^{a} \boldsymbol{\Gamma}^{L} \mathbf{G}^{a} \boldsymbol{\Gamma}^{R}+\mathbf{G}^{r} \boldsymbol{\Gamma}^{L} \mathbf{G}^{r} \boldsymbol{\Gamma}^{R}\right) \\
& -\left[1-n_{L}\right] n_{R}\left(\mathbf{G}^{a} \boldsymbol{\Gamma}^{R} \mathbf{G}^{a} \boldsymbol{\Gamma}^{L}+\mathbf{G}^{r} \boldsymbol{\Gamma}^{R} \mathbf{G}^{r} \boldsymbol{\Gamma}^{L}\right) \\
& \left.\left.+\mathbf{G}^{<} \boldsymbol{\Gamma}^{L} \mathbf{G}^{>} \boldsymbol{\Gamma}^{R}+\mathbf{G}^{<} \boldsymbol{\Gamma}^{R} \mathbf{G}^{>} \boldsymbol{\Gamma}^{L}\right\}\right\} .
\end{aligned}
$$

Com isso derivamos as quatro componentes do ruído $S_{L L}, S_{L R}, S_{R L}$ e $S_{R R}$. Como temos $S_{L L}=S_{R R}=-S_{L R}=-S_{R L}$, ao longo da tese trabalharemos mais com $S_{L L}$, que, em geral, chamaremos simplesmente de $S$. 


\section{Bibliografia}

[1] C. Cohen-Tannoudji, B. Diu, e F. Laloe, Quantum Mechanics, vol. 1, John Wiley e Sons (1977).

[2] L. Kouwenhoven e C. Marcus, Phys. World, 35, June (1998).

[3] A primeira realização experimental de um ponto quântico gerado eletrostaticamente (via eletrodos) sobre uma superfície de GaAs foi de U. Meirav, M. A. Kastner, e S. J. Wind, Phys. Rev. Lett. 65, 771 (1990).

[4] L. V. Keldysh, Sov. Phys. JETP 20, 1018 (1965).

[5] L. P. Kadanoff e G. Baym, Quantum Statistical Mechanics, Green's Function Methods in Equilibrium and Nonequilibrium Problems, Benjamin Cummings Publishing Company (1962).

[6] Y. Meir e N. S. Wingreen, Phys. Rev. Lett. 68, 2512 (1992).

[7] A. P. Jauho, N. S. Wingreen, e Y. Meir, Phys. Rev. B 50, 5528 (1994).

[8] Y. Meir, N. S. Wingreen, e P. A. Lee, Phys. Rev. Lett. 70, 2601 (1993).

[9] P. Zhang et al., Phys. Rev. Lett. 89, 286803 (2002) (cond-mat/0210241).

[10] B. Dong et al., cond-mat/0303093.

[11] P. Zhang, Q.-K. Xue, e X. C. Xie, Phys. Rev. Lett. 91, 196602 (2003).

[12] J. Martinek et al., Phys. Rev. Lett. 91, 127203 (2003).

[13] D. Goldhaber-Gordon et al., Phys. Rev. Lett. 81, 5225 (1998).

[14] S. M. Cronenwett, T. H. Oosterkamp, e L. P. Kouwenhoven, Science 281, 540 (1998).

[15] F. Simmel et al., Phys. Rev. Lett. 83804 (1999).

[16] Single Charge Tunneling: Coulomb Blockade Phenomena in Nanoestructures, editado por H. Grabert e M. H. Devoret, NATO ASI Series, Serie B: Physics 294, Plenum, New York (1992).

[17] T. Inoshita, Science 281, 526 (1998).

[18] S. D. Lee et al., App. Phys. Lett. 77, 2355 (2000). 
[19] J. B. Barner e S. T. Ruggiero, Phys. Rev. Lett. 59, 807 (1987).

[20] L. F. Schelp et al., Phys. Rev. B 56, R5747 (1997).

[21] D. D. Awschalom, M. E. Flatté, e N. Samarth, Sci. American, pg. 53, (June 2002).

[22] S. A. Wolf et al., Science 294, 1488 (2001).

[23] S. Das Sarma et al., Solid State Commun. 119207 (2001).

[24] G. A. Prinz, Science 282, 1660 (1998).

[25] M. Julliere, Phys. Lett. 54A, 225 (1975).

[26] J. S. Moodera et al., Phys. Rev. Lett. 74, 3273 (1995).

[27] M. Tondra et al., J. Appl. Phys. 83, 6688 (1998).

[28] K. Sin et al., J. Appl. Phys. 89, 7359 (2001).

[29] S. S. P. Parkin et al., J. Appl. Phys. 85, 5828 (1999).

[30] J. Barnaś e A. Fert, Phys. Rev. Lett. 80, 1058 (1998).

[31] S. Takahashi e S. Maekawa, Phys. Rev. Lett. 80, 1758 (1998).

[32] W. Rudziński e J. Barnaś, Phys. Rev. B 64, 085318 (2001).

[33] E. Y. Tsymbal et al., Phys. Rev. Lett. 90, 186602 (2003).

[34] M. N. Baibich et al., Phys. Rev. Lett. 61, 2472 (1988).

[35] S. Datta e B. Das, Appl. Phys. Lett. 56, 665 (1990).

[36] Veja por exemplo, G. Meier, T. Matsuyama, e V. Merkt, Phys. Rev. B 65, 125327 (2002).

[37] W. Kuo e C. D. Chen, Phys. Rev. B 65, 104427 (2002).

[38] J. C. Egues, Phys. Rev. Lett. 80, 4578 (1998).

[39] M. Johnson, Science 260, 320 (1993).

[40] P. Recher, E. V. Sukhrukov, e D. Loss, Phys. Rev. Lett. 85, 1962 (2000).

[41] D. Loss e D. P. DiVincenzo, Phys. Rev. A 57, 120 (1998).

[42] D. P. DiVincenzo, Science 270, 255 (1995).

[43] R. de-Picciotto et al., Nature 389, 162 (1997).

[44] R. Landauer, Nature 392, 658 (1998).

[45] Sh. Kogan, Electronic Noise and Fluctuations in Solids, Cambridge University Press, Cambridge (1996). 
[46] Y. P. Li et al., Phys. Rev. B 41, 8388 (1990).

[47] H. C. Liu et al., Phys. Rev. B 51, 5116 (1995).

[48] G. Iannaccone et al., Phys. Rev. Lett. 80, 1054 (1998).

[49] S. S. Safonov et al., Phys. Rev. Lett. 91, 136801 (2003).

[50] E. V. Anda e A. Latgé, Phys. Rev. B 50, 8559 (1994).

[51] H. Birk, M. J. M. de Jong, e C. Schönenberger, Phys. Rev. Lett. 75, 1610 (1995).

[52] A. Nauen et al., Phys. Rev. B 66, R161303 (2002).

[53] A. Nauen et al., cond-mat/0403108.

[54] B. R. Bulka, J. Martinek, e G. Michalek, Phys. Rev. B 60, 12246 (1999).

[55] Y. Tserkovnyak e A. Brataas, Phys. Rev. B 64, 214402 (2001).

[56] R. López e D. Sánchez, Phys. Rev. Lett. 90, 116602 (2003).

[57] H. Haug e A. P. Jauho, Quantum Kinetics in Transport and Optics of Semiconductors, Springer Solid-State Sciences 123 (1996).

[58] F. M. Souza, J. C. Egues and A. P. Jauho, Proceedings of the 26th International Conference on the Physics of Semiconductors (ICPS 26), Edinburgh, 2002, edited by J. H. Davies and A. R. Long. cond-mat/0209263.

[59] F. M. Souza, J. C. Egues, e A. P. Jauho, Coulomb and Spin-flip Effects on Spin Polarized Transport Through a Quantum Dot (a ser submetido).

[60] F. M. Souza, J. C. Egues, e A. P. Jauho, Braz. J. Phys. 34, 565 (2004).

[61] J. C. Egues et al., Phys. Rev. B 64, 195319 (2001).

[62] A. Slobodskyy et al., Phys. Rev. Lett. 90, 246601 (2003).

[63] W. Schottky, Ann. Phys. (Leipzig) 57, 541 (1918).

[64] Ya. M. Blanter and M. Büttiker, Phys. Rep. 336, 2 (2000).

[65] Z. Wang, M. Iwanaga, T. Miyoshi, Jpn. J. Appl. Phys. 37, 5894 (1998).

[66] F. M. Souza e J. C. Egues, Phys. Rev. B 66, (R)60301 (2002).

[67] L. Y. Chen e C. S. Ting, Phys. Rev. B 43, (R)4534 (1991).

[68] Os primeiros trabalhos que aplicaram funções de Green de não equilíbrio no estudo de transporte em pontos quântico foram Y. Meir, N. S. Wingreen, e P. A. Lee, Phys. Rev. Lett. 23, 3048 (1991), Y. Meir e N. S. Wingreen, Phys. Rev. Lett. 68, 2512 (1992), A. P. Jauho, N. S. Wingreen, e Y. Meir, Phys. Rev. B 50, 5528 (1994).

[69] J. Schwinger, J. Math. Phys. 2, 407 (1961). 
[70] G. D. Mahan, Many-Particle Physics, Second Edition.

[71] J. Rammer e H. Smith, Rev. Mod. Phys. 58, 323 (1986).

[72] D. C. Langreth, Linear and Nonlinear Electron Transport in Solids, ed. por J. T. Devreese e E. Van Doren, Plenum, New York (1976).

[73] C. Jacoboni e L. Reggiani, Rev. Mod. Phys. 55, 645 (1983).

[74] A. P. Jauho, Phys. Rev. B 32, 2248 (1985). Veja também A. P. Jauho, Physica B 134, 148 (1985).

[75] Esse Hamiltoniano foi originalmente proposto por W. Rudziński e J. Barnaś, Phys. Rev. B 64, 085318 (2001).

[76] P. Zhang, Qi-Kun Xue, e X. C. Xie, Phys. Rev. Lett. 91, 196602 (2003).

[77] Essa transformação foi proposta por P. Zhang et al., cond-mat/0201465.

[78] Essa equação foi publicada originalmente por nós na referência [58] e em seguida por P. Zhang et al., Phys. Rev. Lett. 89, 286803 (2002) (cond-mat/0210241).

[79] Publicamos originalmente essa equação em [58]. Posteriormente R. Lü e Z. R. Liu (cond-mat/0210350) obtiveram uma equação semelhante para o mesmo sistema (FM-PQ-FM), e J. X. Zhu e A. V. Balatsky, Phys. Rev. B 67, 165326 (2003) (cond-mat/0210003) também encontraram uma equação semelhante a essa para um sistema de um ponto quântico com um modo de fônon. Uma versão escalar (não matricial) dessa equação também pode ser vista em B. Dong e X. L. Lei, J. Phys. Condens. Matter 14, 4963 (2002).

[80] Essa forma de se modelar contatos ferromagnéticos foi originalmente proposta por W. Rudziński e J. Barnas na referência [32].

[81] Essa escolha de $\Gamma_{0}^{L}$ e $\Gamma_{0}^{R}$ é apropriada para pontos quânticos, onde $\Gamma \sim 10-200 \mu \mathrm{eV}$ e $U \sim 1 \mathrm{meV}$. Veja por exemplo os artigos experimentias [13], [15], D. GoldhaaberGordon et al., Nature 391, 156 (1998); P. L. McEuen et al., Phys. Rev. Lett. 66, 1926 (1991).

[82] H. Imamura, S. Takahashi, e S. Mekawa Phys. Rev. B 59, 6017 (1999).

[83] Esses valores correspondem as polarizações do Ni e do Co, respectivamente. Veja R. Meservey e P. M. Tedrow, Phys. Rep. 238, 174 (1994).

[84] Um modelo que utiliza capacitâncias para descrever os efeitos (sobre a corrente) do acúmulo de carga elétrica em um ponto quântico acoplado a eletrodos ferromagnéticos pode ser visto em R. Świrkowicz, J. Barnaś, e M. Wilczyński, J. Phys.: Condens. Matter 14, 2011 (2002).

[85] H. B. Sun e G. J. Milburn, Superlattices and Microstructures 23, 883 (1997).

[86] C. A. Stafford, Phys. Rev. Lett. 77, 2770 (1996). 
[87] A. D. Stone e P. A. Lee, Phys. Rev. Lett. 54, 1196 (1985).

[88] M. Büttiker, Phys. Rev. Lett. 57, 1761 (1986).

[89] Jacek Kossut, Band structure and quanto transporte phenomena in narrow-gap diluted magnetic semiconductors, Semiconductors and Semimentals/Diluted Magnetic Semiconductors, vol. 25, cap. 5, pag.183. Academic Press, San Diego (1988).

[90] J. A. Gaj, Magnetooptical properties of large-gap diluted magnetic semiconductors. In Semiconductors and Semimetals / Diluted Magnetic Semiconductors, Editores J. K. Furdyna e J. Kossut, volume 25 of Semiconductors and Semimentals, capítulo 7, páginas 275-309. Academic Press, San Diego, 1988.

[91] Para uma descrição detalhada desse efeito veja, por exemplo, Henrique J. P. Freire, Magnetotransporte e ferromagnetismo Hall em heteroestruturas semicondutoras magnéticas, tese de doutorado, orientador J. C. Egues, IFSC-USP, (2004).

[92] veja página 270 de N. W. Ashcroft e N. D. Mermin, Solid State Physics, Saunders College Publishing, 1976.

[93] J. C. Egues, Spin-dependent phenomena in Mn-based semiconductors heterostructures (PhD thesis), Department of Physics, The Ohio State University, Columbus, Ohio (1996).

[94] Esse Hamiltoniano foi proposto por L. Bø e Y. Galperin, J. Phys. Condens. Matter 8, 3033 (1996), para estudar corrente e ruído em um sistema de dupla barreira com tunelamento ressonante na presença de campo magnético. Aqui incluímos terminais DMS, e conseqüentemente geramos efeitos dependentes de spin.

[95] Curvas semelhantes a essas do caso NMS foram obtidas por L. Bø e Y. Galperin [veja citação na Ref.[94]]. Também E. V. Anda e A. Latgé [50] encontraram curvas semelhantes para corrente e ruído, estudando transporte em uma heteroestrutura unidimensional de dupla barreira.

[96] O. Sauret e D. Feinberg, Phys. Rev. Lett. 92, 106601 (2004).

[97] W. Belzig e M. Zareyan, Phys. Rev. B 69, (R)140407 (2004)

[98] D. Sánchez, R. López, P. Samuelsson, e M. Büttiker, Phys. Rev. B 68, 214501 (2003).

[99] J. X. Zhu e A. V. Balatsky, Phys. Rev. B 67, 165326 (2003).

[100] T. Novotny, A. Donarini, C. Flindt, e A. P. Jauho, Phys. Rev. Lett. 92, 248302 (2004).

[101] Um sistema como esse, mas sem terminais ferromagnéticos, foi estudado por G. Kießlich, A. Wacker, e E. Schöll, Phys. Rev. B 68125320 (2003).

[102] A.T. Hanbicki et al., App. Phys. Lett. 80, 1240 (2002). 
[103] B. T. Jonker et al., Phys. Rev. B 62, 8180 (2000).

[104] Para uma breve discussão sobre a representação matricial de funções de Green ordenada no contorno veja G. D. Mahan, Many-Particle Physics, Second Edition, Cap. 2, Sec. 2.9 . 